NBS

Pubil -

cations

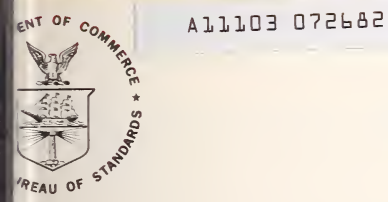

NBS SPECIAL PUBLICATION $\mathbf{5 3 3}$

U.S. DEPARTMENT OF COMMERCE / National Bureau of Standards.

\title{
Characterization of Particles
}

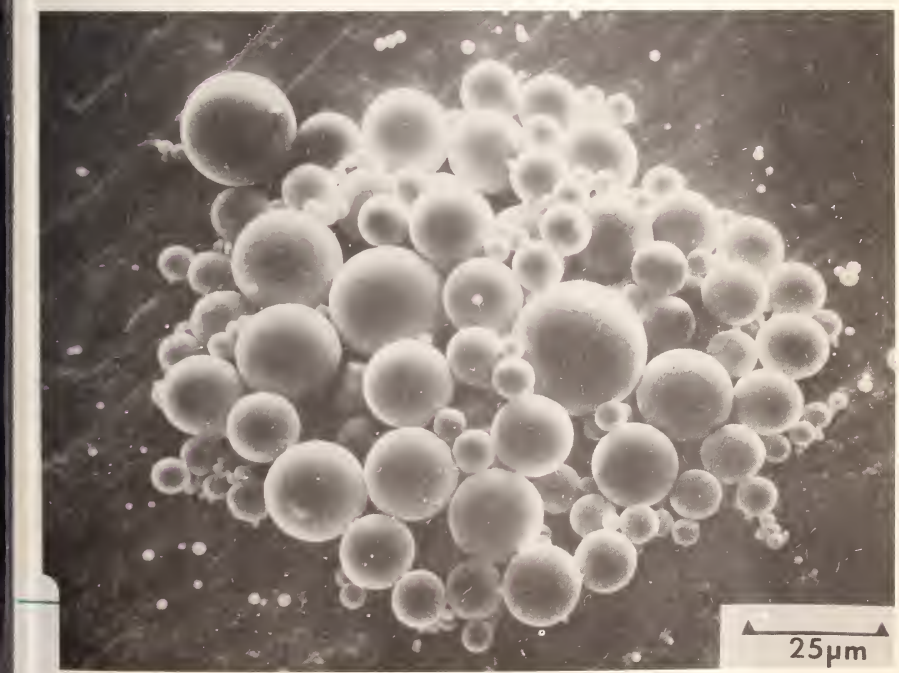




\section{NATIONAL BUREAU OF STANDARDS}

The National Bureau of Standards' was established by an act of Congress on March 3, 1901. The Bureau's overall goal is to strengthen and advance the Nation's science and technology and facilitate their effective application for public benefit. To this end, the Bureau conducts research and provides: (1) a basis for the Nation's physical measurement system, (2) scientitic and technological services for indusiry and government, (3) a technical basis for equity in trade, and (4) technical services to promote public safety. The Bureau's technical work is performed by the National Measurement Laboratory, the National Engineering Laboratory, and the Institute for Computer Sciences and Technology.

THE NATIONAL MEASUREMENT LABORATORY provides the national system of physical and chemical and materials measurement; coordinates the system with measurement systems of other nations and furnishes essential services leading to accurate and uniform physical and chemical measurement throughout the Nation's scientific community, industry, and commerce; conducts materials research leading to improved methods of measurement, standards, and data on the properties of materials needed by industry, commerce, educationat institutions, and Government; provides advisory and research services to other Government agencies; develops, produces, and distributes Standard Reterence Materials; and provides calibration services. The Laboratory consists of the following centers:

Absolute Physical Quantities² - Radiation Research - Thermodynamics and Molecular Science - Analytical Chemistry - Materials Science.

THE NATIONAL ENGINEERING LABORATORY provides technology and technical services to the public and private sectors to address national needs and to solve national problems; conducts research in engineering and applied science in support of these efforts; builds and maintains competence in the necessary disciplines required to carry out this research and technical service; develops engineering data and measurement capabilities; provides engineering measurement traceability services; develops test methods and proposes engineering standards and code changes; develops and proposes new engineering practices; and develops and improves mechanisms to transfer results of its research to the ultimate user. The Laboratory consists of the following centers:

Applied Mathematics - Electronics and Electrical Engineering? - Mechanical Engineering and Process Technology $y^{2}$ - Building Technology - Fire Research Consumer Product Technology — Field Methods.

THE INSTITUTE FOR COMPUTER SCIENCES AND TECHNOLOGY conducts research and provides scientific and technical services to aid Federal agencies in the selection, acquisition, application, and use of computer technology to improve effectiveness and economy in Government operations in accordance with Public Law 89-306 (40 U.S.C. 759), relevant Executive Orders, and other directives; carries out this mission by managing the Federal Information Processing Standards Program, developing Federal ADP standards guidelines, and managing Federal participation in ADP voluntary standardization activities; provides scientific and technological advisory services and assistance to Federal agencies; and provides the technical foundation for computer-related policies of the Federal Government. The Institute consists of the following centers:

Programming Science and Technology - Computer Systems Engineering.

'Headquarters and Laboratories at Gaithersburg, MD, unless otherwise noted;

mailing address Washington, DC 20234

Some divisions within the center are located at Boulder, CO 80303. 


\section{Characterization of Particles}

Proceedings of the Particle Analysis Session of the 13th Annual Conference of the

Microbeam Analysis Society held at Ann Arbor, Michigan, June 22, 1978

RATOZTAR BUREAD

OF TRANDARDS

LIBRARY

APR 251980

$10 t \mathrm{ECC}$

. US7

110.523

Kurt F. J. Heinrich, Editor

Center for Analytical Chemistry

National Measurement Laboratory

National Bureau of Standards

Washington, D.C. 20234

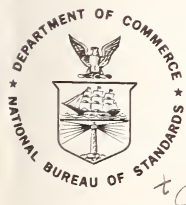

U.S. DEPARTMENT OF COMMERCE, Philip M. Klutznick, Secretary

Luther H. Hodges, Jr., Deputy Secretary

Jordan J. Baruch, Assistant Secretary for Productivity, Technology, and Innovation NATIONAL BUREAU OF STANDARDS, Ernest Ambler, Director 


\section{Library of Congress Catalog Card Number: 80-600033}

\section{National Bureau of Standards Special Publication 533}

Nat. Bur. Stand. (U.S.), Spec. Publ. 533, 222 pages (Apr. 1980)

CODEN: XNBSAV

U.S. GOVERNMENT PRINTING OFFICE

WASHINGTON: 1980

For sale by the Superintendent of Documents, U.S. Government Printing Office, Washington, D.C. 20402 Price $\$ 6.50$

(Add 25 percent for other than U.S. mailing). 
The Center for Analytical Chemistry of the National Bureau of Standards, National Measurement Laboratory, is concerned with the development of new techniques of analysis as well as the improvement of existing methods of chemical analysis. Since one of its goals is to further the dissemination of knowledge, the NBS Center for Analytical Chemistry has published a series of Special Publications covering areas of analytical chemistry such as Quantitative Electron Probe Microanalysis (NBS Special Publication 298), Secondary Ion Mass Spectrometry (NBS Special Publication 427), and The Use of Monte Carlo Calculations in Electron Probe Microanalysis and Scanning Electron Microscopy (NBS Special Publication 460). These publications are available from the Superintendent of Documents, Government Printing Office, Washington, DC 20402.

The subject of this volume, the characterization of particles, is of great importance to many scientific and technological fields, including metallurgy, energy generation, pollution prevention, recycling of materials, and biology. This publication should serve to document the current state of particle characterization, and should therefore be a valuable reference to workers in the area.

J. D. Hoffman, Director

National Measurement Laboratory 


\section{PREFACE}

The characterization of microscopic particles includes the determination of size and shapes as well as elementary and molecular chemical analysis. In the Gas and Particulate Science Division of the Center for Analytical Chemistry, NBS, and in other Divisions of the National Measurement Laboratory, several advanced instrumental techniques were applied to these ends. The editor of this publication was invited to organize and chair at the Conference of the Microbeam Analysis Society (Ann Arbor, June 22, 1978) a special session on the characterization of microscopic particles. Parts of the materials contained in papers 3-12 were orally presented at this session.

The materials here presented have been brought up to date and three papers not orally presented in Ann Arbor were added to this publication in order to obtain a fuller coverage of the subject. Since a written publication covering this subject was not previously available, it can be expected that this NBS Special Publication will be found useful by a wide range of technologists interested in particulate matter. 
This document contains a series of invited papers on the subject of Particle Characterization. Part of the material presented here was presented orally at the Thirteenth Annual Conference of the Microbeam Analys is Society, at Ann Arbor, Michigan, on June 22, 1978. The publication describes microscopic and analytical techniques for the characterization of single microscopic particles. Applications of these techniques to problems of general interest are also included.

Key words: Biological fluids; electron microscopy: electron microprobe; ion probe; 1ight microscopy; microanalysis; microdroplets; particles; particulate matter. 
FOREWORD.

PREFACE

ABSTRACT.

CHARACTERIZATION OF PARTICLES

K. F. J. Heinrich

OPTICAL MICROSCOPY OF PARTICLES

E. B. Stee1

VARIATION IN X-RAY INTENSITY RATIOS USED TO IDENTIFY ASBESTOS FIBERS. John C. Russ

QUANTITATIVE ANALYSIS OF SMALL PARTICLES USING WAVELENGTH AND ENERGY DISPERSIVE

SYSTEMS IN AN ELECTRON BEAM INSTRUMENT. John Gavrilovic

PROCEDURE FOR THE QUANTITATIVE ANALYSIS OF SINGLE PARTICLES WITH THE ELECTRON PROBE . J. A. Sma11, K. F. J. Heinrich, D. E. Newbury, R. L. Myklebust, and C. E. Fiori

MONTE CARLO ELECTRON TRAJECTORY SIMULATION - AN AID FOR PARTICLE ANALYSIS . . . . . . Dale E. Newbury, Robert L. Myklebust, Kurt F. J. Heinrich, and John A. Small

QUANTITATIVE CHARACTERIZATION OF PARTICULATES BY SCANNING AND HIGH VOLTAGE

ELECTRON MICROSCOPY .

R. J. Lee and R. M. Fisher

CHARACTERIZATION OF COAL GASIFICATION PARTICULATES BY SEM, EDS, AES, XPS, AND

ITS RELEVANCE TO INHALATION TOXICOLOGY.

D. L. Davidson and E. M. Gause

ACCURACY OF ELECTRON MICROPROBE ANALYSIS OF BIOLOGICAL FLUIDS: CHOICE OF STANDARD

SOLUTIONS, AND RANGE OF LINEARITY OF THE CALIBRATION CURVES . . . . . . . . . . . . 10 Nicole Roinel, Lucienne Meny, and Jean Henoc

APPLICATION OF AUGER-ELECTRON SPECTROSCOPY AND X-RAY PHOTOELECTRON SPECTROSCOPY

TO THE CHARACTERIZATION OF POLLUTANT PARTICLES. C. J. Powel1

SECONDARY ION MASS SPECTROMETRY FOR THE ANALYSIS OF SINGLE PARTICLES. . . . . . . . . 13S Dale E. Newbury

SCOPE AND LIMITATIONS OF SINGLE PARTICLE ANALYSIS BY RAMAN MICROPROBE SPECTROSCOPY. . . 15: Edgar S. Etz and John J. Blaha

LASER MICROPROBE MASS ANALYSIS (LAMMA) IN PARTICLE ANALYSIS . . . . . . . . . . . . 19! R. Kaufmann and $P$. Wieser

Disclaimer: In order to adequately describe materials and experimental procedures, it was occasionally necessary to identify commercial products by manufacturer's name or label. In no instance does such identification imply endorsement by the National Bureau of Standards nor does it imply that the particular products or equipment is necessarily the best available for that purpose. 
tional Bureau of Standards Special Publication 533. This Publication Originated from a ecial Session on Particle Analysis, 13th Annual Conference of the Microbeam Analysis ciety, Ann Arbor, MI, June 22, 1978. (Issued April 1980)

\title{
CHARACTERIZATION OF PARTICLES
}

\author{
K. F. J. Heinrich* \\ Gas and Particulate Science Division \\ National Bureau of Standards \\ Washington, D. C. 20234
}

\section{Introduction}

In particles of microscopic dimensions, many properties vary from those of macroscopic lids and liquids. This is mainly due to the much larger ratio of surface to volume and , the correspondingly large surface effects. Microscopic particulates thus resemble a fferent state of aggregation.

Particulate materials play important roles in all aspects of life: soils, sands, igments, solid insecticides, as well as living cells and celiular organelles, bacteria, id viruses, to only cite a few, are all constituted of microscopic particles. In addition, arious human activities cause the production and/or dissemination of particles of potenially unfavorable economic or health effects. Examples include particulate air pollutants - sch as fly ash and asbestos fibers, fallout particles, wear particles in oils, and articulate pollutants of waters.

The macroscopic characterization of such particulates by diverse means (including the etermination of particle size distribution and chemical analysis) is a subject of enormous readth which we will not consider here. In many instances, however, it is necessary to aracterize individual single particles and to distinguish them from particles having diferent origin, properties, and/or significance. In special sessions of the Thirteenth nnual Conference of the Microbeam Analysis Society [1] ${ }^{1}$ (Ann Arbor, Mich., June 19-23, 978), eleven papers dealing with studies of particles were presented. Selected communicaions covering the material presented in these papers, and extensively revised since the AS Conference, are the subject of the present publication.

\section{Observable Characteristics of Prime Interest}

Among the characteristics of particles which may be of interest to the investigator, re size, shape, optical properties, composition, structure, and heterogeneity.

The relevance of particle size to aerodynamic properties, respirability, and, in ertain types of particles, toxicity, is widely discussed in literature. Shape has imilar implications, particularly in the aspects of toxicity of fibrous minerals [2]. his and other microscopic aspects are easily observable in microscopes of appropriate patial resolution. Hence, microscopy in its various forms plays a prominent role in article identification.

A second area of great interest is compositional analysis, either on an elemental Papers 3-10), isotopic (Paper 10), or molecular (Paper 11) leve1. Finally, the structure f crystalline lattices can be characterized by the optical microscope or by electron iffraction.

*New address: Office of International Relations, National Bureau of Standards, Washington, D.C. 20234.

IFigures in brackets indicate literature references at the end of this paper. 
It is important to realize that the size range of particles considered to be microscopic extends from a few $\mu \mathrm{m}$ to $1 \mathrm{~mm}$. But diverse techniques have different size ranges of applicability, and must therefore be chosen accordingly (figure 1). The difficulties of microscopic and spectroscopic identification, as well as the cost of analysis and of the required equipment, increase sharply with decreasing particle size. Therefore, most of the published work deals with particles of diameter above $1 \mu \mathrm{m}$, although there is enormous interest in the particles which are smaller than $1 \mu \mathrm{m}$.

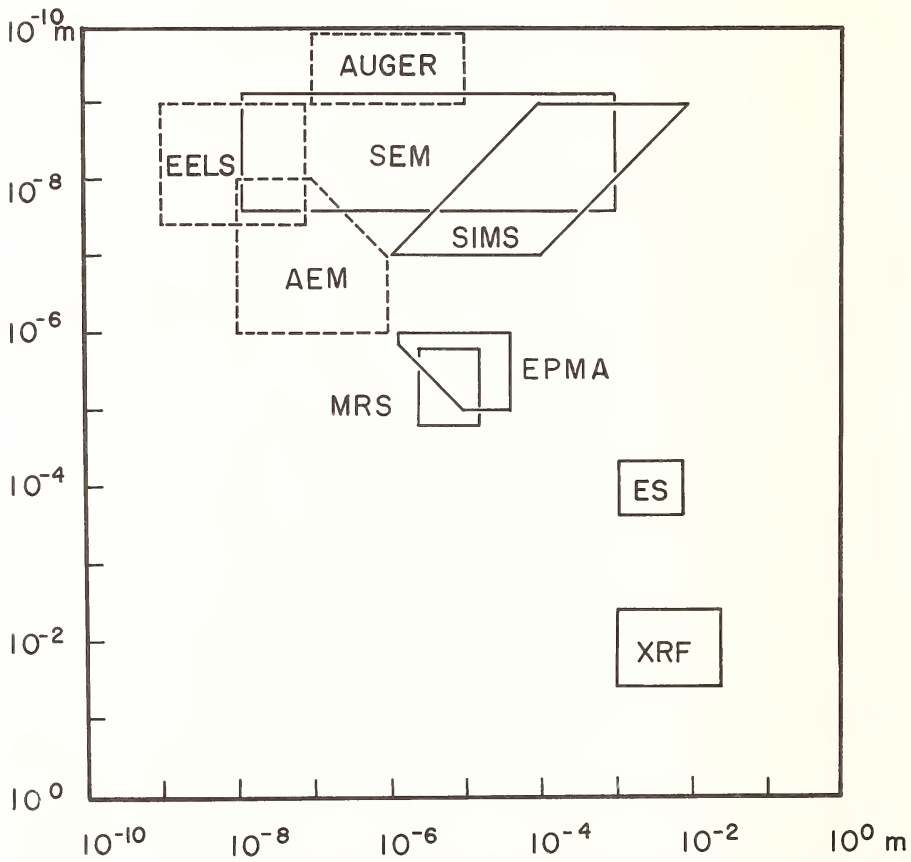

Figure 1. Typical ranges of dimensions of excited regions with several techniques: AEM analytical electron transmission microscopy; AUGER - electron probe Auger microanalysis - EELS - electron energy low spectroscopy; EPMA - electron probe microanalysis; MRS - Micro-Raman spectroscopy; SEM - scanning electron microscopy; SIMS - secondary ion mass spectroscopy (ion probe microanalysis), XRF - x-ray fluorescence. The horizontal scale represents the diameter of the analyzed region in the plane of the specimen surface and the vertical scale represents depth. 
The inclusion of paper 9, of Roinel et al., deserves some comment, since it deals with re analysis of small drops of biological fluids. Although these drops are typically 3-100 $\mu \mathrm{m}$ in diameter, the dehydration of the fluid produces small ( $\sim \mu \mathrm{m}$ diameter) articles of solid residue, and the calibration problems discussed in this paper are lated to the dimensions of these particles. Some aspects of relevance to this topic are iso included in the paper by Newbury et al.

\section{References}

1] Proceedings, 13th Annual Conference, Microbeam Analysis Society of America, Ann Arbor, Mich., June 19-23, 1978, obtainable from K. F. J. Heinrich, Bldg. 222, Rm. A-119, Nat. Bureau of Standards, Washington, D. C. 20234.

2] Proceedings of Workshop on Asbestos: Definitions and Measurement Methods, NBS, Gaithersburg, Md., July 18-20, 1977, C. C. Gravatt, P. D. LaFleur, K. F. J. Heinrich, eds., NBS Special Publication 506, U. S. Dept. of Commerce.

In order to adequately describe materials and experimental procedures, it was occasion$11 y$ necessary to identify commercial products by manufacturer's name or label. In no nstance does such identification imply endorsement by the National Bureau of Standards nor oes it imply that the particular product or equipment is necessarily the best available or that purpose. The opinions of authors outside of NBS do not reflect that of NBS. 

ational Bureau of Standards Special Publication 533. This Publication Originated from a becial Session on Particle Analysis, 13th Annual Conference of the Microbeam Analysis sciety, Ann Arbor, MI, June 22, 1978. (Issued Apri1 1980)

\title{
OPTICAL MICROSCOPY OF PARTICLES
}

\author{
E. B. Stee ${ }^{*}$ \\ Bureau of Mines \\ Avondale Metallurgy Research Center \\ Avondale, Maryland 20782
}

\begin{abstract}
The optical microscope is one of the oldest and most effective methods for studying the morphology of particulates and for determining particle identity. A wide range of particle sizes and types are within the light microscope's useful limits. Particles as small as approximately $0.25 \mu \mathrm{m}$ can be resolved and optical data can be collected on most particles larger than a few micrometers in diameter. Particle size, orientation, contrast, the depth of field, the similarity of optical properties among different types of particulates, and the microscopists' experience can all play an important role in applying the light microscope to the analysis of particulates.
\end{abstract}

\section{Introduction}

The light microscope is an excepiionally versatile tool used in a wide variety of article applications. Whether one's field of interest is with nanoplankton, asbestos, or ome other area, the microscope is often the first and most efficient means of studying articulates. The purpose of this paper is to look at the capabilities and limitations of he light microscope as it is applied to the study of particulates. The actual techniques entioned are covered in detail in the references provided.

There are two basic types of information that may be obtained by optical microscopy hen studying particulates. These are the morphology and the optical properties of the articles. A variety of data can be collected using the particle morphology, including ample size distributions $[1]^{1}$, crystal growth and fracture information, and particle idenification. The optical properties are generally measured to identify partic?es or observe he orientation of particles, especially in nonbiologic and crystalline samples.

\section{Discussion}

The microscope has many characteristics which are well suited to the study of particuates. Microscopy is generally a nondestructive technique which allows the same particles ibserved on the microscope to be analyzed by some other technique, such as the laser Raman ir electron microprobes. Sample preparation is usually relatively simple and quick, nvolving mounting the particles on a glass slide in a refractive index oil or immersion ledium. The microscope has a large field of view and is capable of quickly scanning a large iample area. The approximate size of the field of view observed with the various objectives s given in Table 1. This scanning capability gives the microscope very good sensitivity that is mainly a function of the amount of time spent looking for a particle. A wide variety of particle sizes, from several millimeters to less than a micrometer, can be observed with only minor changes in the instrument. A number of identification techniques can be adapted

Current adress: Center for Analytical Chemistry, National Bureau of Standards, Washington, D.C. 20234

IFigures in brackets indicate literature references at the end of this paper. 
Table 1. Characteristics for some common light microscope objectives.

\begin{tabular}{|c|c|c|c|c|}
\hline Magnification & $\begin{array}{l}\text { Numerical } \\
\text { Aperture }\end{array}$ & $\begin{array}{l}\text { Depth of } \\
\text { Field } \\
(\mu \mathrm{m})\end{array}$ & $\begin{array}{c}\text { Diameter }{ }^{a} \text { of } \\
\text { Field } \\
(\mathrm{mm})\end{array}$ & $\begin{array}{c}\text { Resolving } \\
\text { Power } \\
(\mu \mathrm{m})\end{array}$ \\
\hline $3 x$ & 0.08 & 50 & 9 & 5 \\
\hline 10 & 0.25 & 8 & 2 & 1.3 \\
\hline 50 & 0.85 & 1 & 0.5 & 0.4 \\
\hline 100 & 1.3 & 0.4 & 0.4 & 0.25 \\
\hline
\end{tabular}

\footnotetext{
$\overline{{ }^{a} \text { Approximate }}$ value with $10 x$ oculars.

${ }^{b}$ Approximate value in green light.
}

to meet the special problems presented by the sample [2-4]. A permanent record and illustration can be made through the use of photomicrography $[5,6]$. The generally high contrast of specimens viewed on the microscope makes it a good technique for automated particle sizing and counting. Microscopy can be used quantitatively to find the relative abundance of specific types of particles in a sample by combining the optical information with a counting technique, such as point counts or area counts. In addition, the qualitative chemistry can be determined using the methods of chemical microscopy, and phase stability can be defined for some particles using a hot stage $[7,8]$.

The limitations of the optical microscope are due to the nature of visible light and are often dependent on the size and the specific optical and physical properties of the particles found in a sample.

In order to observe and measure the size and optical parameters of particles, there must be adequate resolution, contrast, and depth of field for the size and type of particle in the sample.

Most microscopes are capable of using a number of optical lenses which yield useful magnifications of approximately $15 \mathrm{X}$ to $1500 \mathrm{X}$. Each objective lens has a numerical aperture (N.A.) which will determine its resolving power at a given wavelength of light [9]. Table 1 gives the resolution in green light for some common objectives. It can be seen that the best resolution in green light is about $0.25 \mu \mathrm{m}$. While an observer may be able to see objects somewhat smaller than $0.25 \mu \mathrm{m}$ across, they will not be resolved and accurate measurement of these or smalier particles is not possible with the light microscope. In general, if the particles are smaller than about $0.5 \mu \mathrm{m}$, electron microscopy is the better tool for particle sizing.

The observation and measurement of particles also requires that they be in a definite region of focus. The maximum depth of this region is controlled by the numerical aperature of the objective lens and is called the depth of field. Table 1 shows that the depth of focus decreases with increasing magnification or N.A. An example of some of the problems associated with shallow depth of field are seen in figures 1 and 2 . Figure 1 shows the same field of view taken at three different depths. Particles appear and disappear as the microscope is focussed up and down. This makes it possible to miss particles of interest when performing scans of areas, unless it is carefully observed over the full depth of the sample mount. Figure 3 shows a photomicrograph of a coccolith in which the full depth of the individual particle cannot be focussed, so that the edges of the coccolith are in focus at one point and the interior of the particle at another point. If this type of focus is critical, then the scanning electron microscope, with its superior depth of focus, could be used to observe the particle. 
a

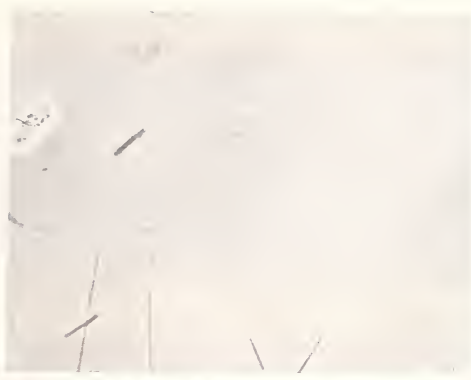

b

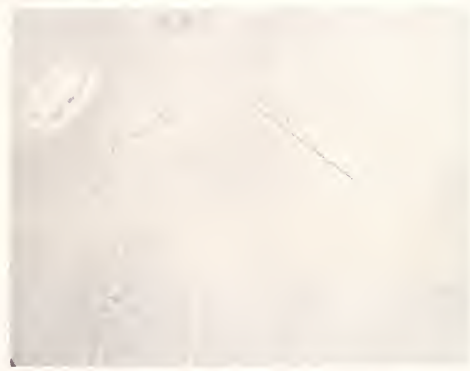

C

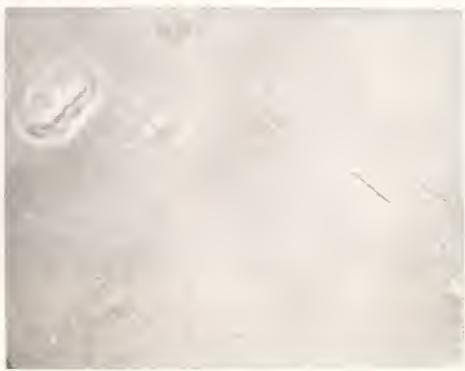

gure 1. Photomicrographs of the same area of a dissolved filter on which asbestos fibers were collected. The light microscope is one of the few instruments capable of observing through the full depth of a cleared or transparent filter. However, as one focusses down through the filter from part (a) to part (c), fibers appear and disappear, making it possible to miss fibers or other particles in the sample. Also, the depth of field is often insufficient to see even the individual fiber bundles, making their measurement and observation difficult. Photomicrographs were taken using phase contrast $40 \mathrm{X} / 0.65 \mathrm{NA}$ objective. 


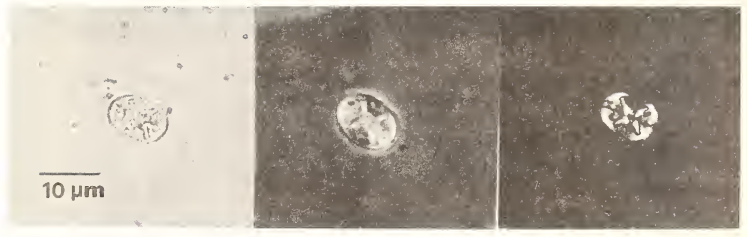

a

b

C

Figure 2. Photomicrographs of a coccolith, a fossil marine plankton, as seen under brightfield illumination (a), phase contrast (b), and crossed polarized light (c) conditions. Photomicrographs were taken using a 90K/1.4 NA polarized light objective for (a) and (c), and a 100X/1.3 NA phase contrast objective for (b).
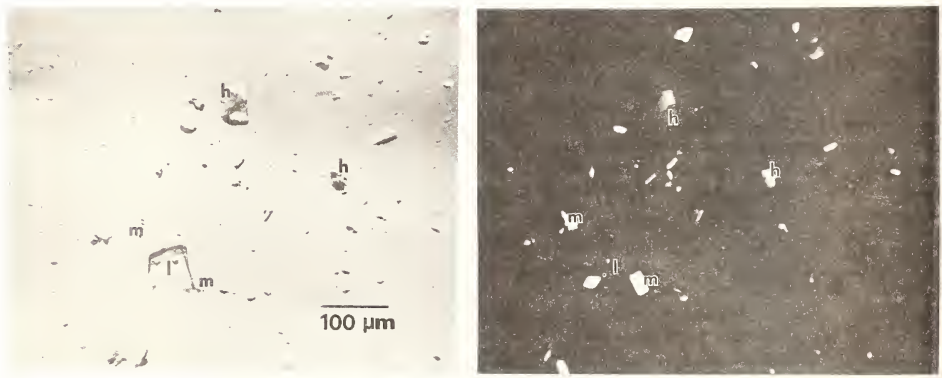

a

b

Figure 3. Photomicrograph (a) exhibits the effect of relief on observation in the light microscope. The refractive index of the mounting medium matches that for the particles labelled $\mathrm{m}$, which are therefore almost invisible in brightfield illumination. Particles $h$ and 1 have refractive indices that are higher and lower than the mounting medium and therefore have reasonably good relief. Photomicrograph (b) shows the same field of view as that shown in (a). The field in (b) is viewed through crossed polarizers and the particles marked $m$ are now easily' visible, while the isotropic particle 1 is not visible in this mode of observation. 
In addition to resolution and depth of focus, enough contrast must be present between particle and the mounting medium to make observation of the particle practical. In leral, the higher the magnification the lower the contrast. Also, the greater the difence in the refractive index between the mounting medium and the particle, the greater contrast. This latter contrast mechanism is referred to as relief. Figure 3 shows a imon case where the relief is quite low, that is, when determining the refractive index a particle. This is done by immersing the particle in an oil with the same or very illar refractive index as the particle. In such cases some other contrast mechanism is aded to increase the contrast and allow the grain to be observed. These mechanisms clude phase contrast, interference contrast, fluorescence, dark field illumination, and sssed polarized light [10]. Each of these methods can show different details in the phology or optical properties of the sample. Some of these contrast mechanisms are wW in figures 2 and 3.

Once the particle is observed, it may be possible to identify it. Many particles may recognized by morphology alone, such as the coccolith shown in figure 3 . This type of entification is directly related to the observer's experience and is therefore as much ; as it is science. When identifying particles by their morphology, it is often helpful compare the unknown to a known sample or a photomicrograph of a known sample. (Such as se found in McCrone's Particle Atlas [11].) Another way to identify particulates is to asure their optical properties using the polarizing light microscope. The optical operties that are measurable in transmitted light are listed in Table 2.

Table 2. Optical and physical properties observable using the polarizing transmitted light microscope.

\author{
Refractive Index or Indices \\ Transmitted Color \\ Relative Light Absorption \\ Optic Axial Angle \\ Extinction Angle \\ Dispersion \\ Inclusions (orientation or type) \\ Morphology (cleavage or fracture) \\ Sign of Elongation \\ Twinning \\ Birefringence \\ Optic Sign \\ Optical Indicatrix
}

Using all the properties listed in Table 2 it is almost always possible to unambigusly identify particulates. However, it is often not possible to measure all of these tical parameters on every grain in a sample. The particle's size or orientation may not low some of the properties to be measured and if optically similar grains are present in e sample, then an unambiguous identification may not be possible.

The most important optical parameter is the refractive index or indices of the rticle. The number of refractive indices for a particle depends on its crystal symtry. Amorphous substances, such as glass, and crystals with isometric symmetry have one fractive index and are called isotropic substances. Other crystalline material will have o or three refractive indices, again depending on their crystal symmetry. These nonotropic particles must be oriented in specific directions in order to accurately measure eir refractive indices. It is common for the grains in a sample to have preferred 
orientations due to cleavage or crystal faces or to have random orientations that do not allow the specific light vibrations necessary for accurate indices measurements to be observed. In these cases approximate values close to the high and low refractive indices for the particles are measured. Often this less accurate index determination is still sufficient to identify the type of particle, or at least distinguish it from the other types of particles in the sample. In other cases this lack of accuracy may cause the index measurement to overlap with the data from other types of particles making unambiguous identification difficult. There are ways to get around the orientation problems, such as simply tapping the coversiip so that the particle orientation changes, or using a universal stage so that the grain orientation can be systematically manipulated [12].

Another factor affecting the measurement of the optical properties and therefore the identification of many grains is the size of the particles. When the particle diameter is below approximately $5-10 \mu \mathrm{m}$ it becomes difficult to measure many of the optical parameters. Birefringence becomes weak or negligible, the orientation of the grain becomes difficult to determine, as do the refractive indices. Below about $1 \mu \mathrm{m}$ in diameter, the optical parameters are impractical to measure and particle identification is probably not possible.

The preceding discussion applies mainly to particles that are transparent to light. For opaque particles, the reflected light microscope must be used to measure the optical properties. These properties include such things as the reflectivity, color, and anisotropy of the particle $[13,14]$. The reflected light data are not as definitive as the transmitted light data an identification is largely dependent on the operator's experience. To make these measurements, the particles must be prepared as flat polished specimens, which is often not practical for particulate samples. However, because of the sample preparation method, these optical data are especially easy to correlate with electron microprobe data.

\section{Conclusions}

The optical microscope is an efficient and nondestructive tool which can be applied to many particulate analysis problems. The microscope has a relatively low initial cost and very little upkeep cost with almost no "down" time. Combining this with its relatively rapid sample preparation and analysis time makes microscopy a very efficient technique. It can be used as an independent technique within the limits discussed above or due to its nondestructive nature it can be used as a complimentary technique with other types of particle analysis.

\section{References}

[1] Cadle, R. D., Particle Size: Theory and Industrial Applications, Reinhold Co., New York, NY (1965).

[2] Bloss, F. D., An Introduction to the Methods of Optical Crystallography, Holt, Rinehart, and Winston, Inc., New York, NY (1961).

[3] Kerr, P. F., Optical Mineralogy, McGraw-Hi11, Inc., New York, NY (1977).

[4] Crossman, G. C., Ana1. Chem., 20, 976 (1948).

[5] Eastman Kodak Company, Photomicrography, Rochester, NY (1974).

[6] Allen, R. M., Photomicrography, Van Nostrand Co., Inc., New York, NY (1943).

[7] Chamot, E. M. and Mason, C. W., Handbook of Chemical Microscopy, John Wiley \& Sons, Inc., New York, NY (1940).

[8] JuTian, Y. and McCrone, W. C., "Accurate use of hot stages," Microscope, 19, 225-234 (1971). 
9] Longhurst, R. S., Geometrical and Physical Optics, Longmans, Green and Co., Ltd., London (1964).

0] Needham, G. H., The Practical Use of the Microscope, Charles C. Thomas, Publisher, Springfield, I1i. (1958).

1] Mccrone, W. C. and Delly, J. G., The Particle Atlas, Ann Arbor Science Publishers Ann Arbor, Michigan (1973).

2] Emmons, R. C., "The Universal Stage," Mem. Geol. Soc. Amer., No. 8 (1943).

3] Cameron, E. N., Ore Microscopy, John Wiley \& Sons, Inc., New York, NY (1961).

14] Freund, H., Applied Ore Microscopy, Macmillan Co., New York, NY (1966). 
ional Bureau of Standards Special Publication 533. This Publication Originated from a cial Session on Particle Analysis, 13th Annual Conference of the Microbeam Analysis :iety, Ann Arbor, MI, June 22, 1978. (Issued Apri1 1980)

\title{
VARIATION IN X-RAY INTENSITY RATIOS USED TO IDENTIFY ASBESTOS FIBERS
}

\author{
John C. Russ ${ }^{1}$ \\ EDAX Laboratories \\ P. 0. Box 135 \\ Prairie View, IL 60069
}

Identification of asbestos fibers and other small particulate matter observed in TEM 1 STEM is often based in whole or in part on the ratios of elemental intensities [1-3] . underlying principle in this method is the linear relationship between concentration ;io and intensity ratio, which has been proposed by many authors, some of whom measure proportionality factors [4] and others calculate them from quasi-theoretical relationps [5].

To be able to routinely apply this method to confidently identify particles, it is cessary to evaluate the magnitude of variation that may be encountered, and the extent to ich it may allow for confusion. We have analyzed a series of asbestos minerals and others ich potentially could be mistaken for them. Table 1 lists the concentrations of the major ements which would normally be chosen for analysis, and figure 1 shows the concentration cios $\mathrm{Mg} / \mathrm{Si}$ and $\mathrm{Fe} / \mathrm{Si}$, indicating that the materials are indeed all distinct.

Table 1. Bulk concentration (W/o) of major elements (by XRF).

\begin{tabular}{|c|c|c|c|}
\hline Mineral & Percént Mg & Percent $\mathrm{Si}$ & Percent $\mathrm{Fe}$ \\
\hline Amosite & $2.4-4.0$ & $22.4-23.1$ & $28.4-34.2$ \\
\hline Anthophyllite & $12.7-17.2$ & $21.5-27.1$ & $4.3-8.4$ \\
\hline Chrysotile & $24.0-25.9$ & $18.3-19.6$ & $1.4-2.4$ \\
\hline Crocidolite & $0.6-1.60$ & $22.7-23.8$ & $27.9-29.5$ \\
\hline Talc & $18.3-19.2$ & $28.1-29.6$ & $0.3-1.0$ \\
\hline Tremolite & $14.4-14.8$ & $26.5-29.6$ & $1.0-3.3$ \\
\hline
\end{tabular}

It is important to note that the samples available did not encompass the complete Ige of elemental composition for a given asbestos mineral. (e.g., Crocidolite from livia contains much more magnesium than amosite from South Africa, although Table 1 shows gher magnesium levels in amosite than in crocidolite [6].) The additional variation ssible should be borne in mind, as it will increase the uncertainty to be described.

The uncertainty of mineral identification must also be understood to include confusion tween asbestos-like and other minerals which happen to contain the same ratios of major ements, in this case Mg, Si, and Fe. Even though the actual concentrations, crystal ructure, degree of hydration, and so forth may be quite different, it is only elemental tios that are used in this method to make the determination. It should also be pointed $t$ that this general approach is often used for the identification of other small parcles besides the asbestos-like ones being described here. The same types of problems and tential confusion are present in those situations as well.

resent address: North Carolina State University, Engineering Research Serviced Division, 158 Burlington Engineering Laboratories, Box 5995, Raleigh, North Carolina 27650.

igures in brackets indicate the literature references at the end of this paper. 


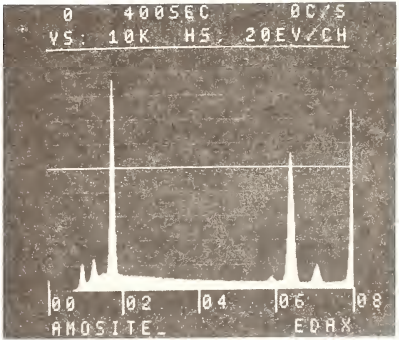

la

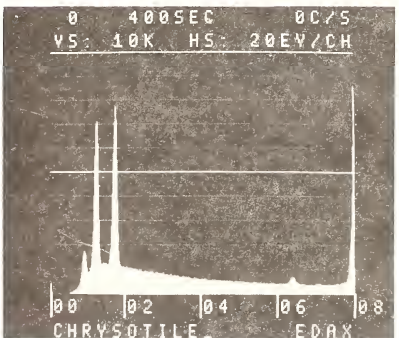

IC

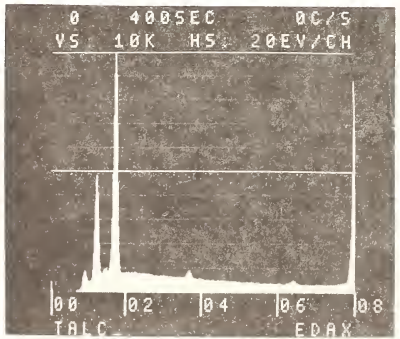

le

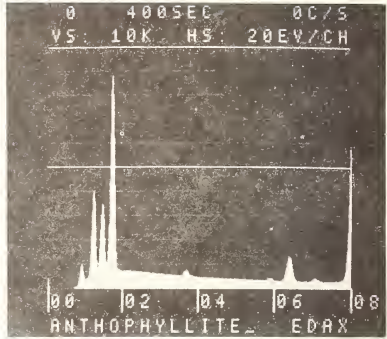

Ib

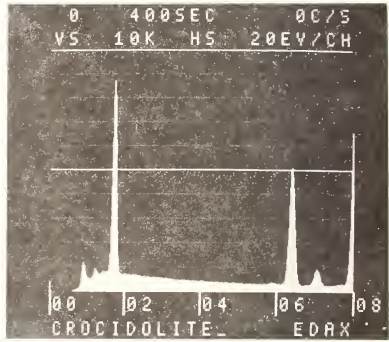

Id

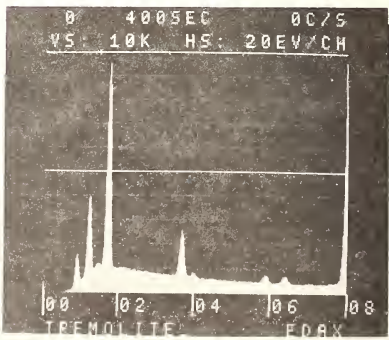

If

Figure $1(a-f)$. Representative spectra from various mineral particles. 
Figure 2 shows representative electron-excited spectra of particles at $80 \mathrm{kV}$ in the STEM. From such spectra, measured for 400 seconds to obtain good counting statistics, we determined the factors $k_{x S i}$ defined by the relationship:

$$
\frac{\text { Conc }_{x}}{\text { Conc }_{\text {Si }}}=k_{x \text { Si }} \frac{\text { Inten }_{x}}{\text { Inten }_{\text {Si }}}
$$

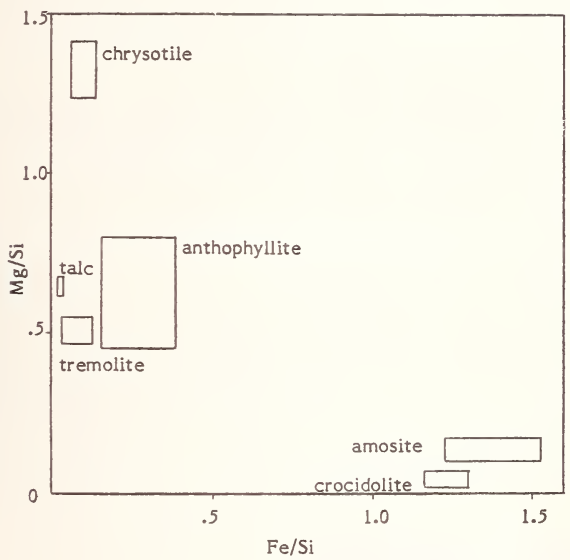

Figure 2. Plot of concentration ratios for minerals (see Table 1).

Since the $k_{x S i}$ factor is not truly a constant, but depends to some extent on particle size, orientation, and matrix composition, we measured a series of particles ranging from approximately 0.1 to $5 \mu \mathrm{m}$, and for the larger particles measured spectra both with the beam centered on the fiber or particle and with the beam scanning to cover the entire area (or for some of the longer fibers, a representative section). The total peak area above a fitted background curve was integrated and the mean bulk composition used to calculate $k_{x S i}$ factors, which ranged as shown in Table 2.

Table 2. Calculated $k_{x S i}$ factors for different minerals and particle sizes.

Mineral

Amosite

Anthophyllite

Chrysotile

Crocidolite

Talc

Tremolite
$\mathrm{k}_{\mathrm{MgSi}}$

not determined

$1.30-1.58$

$1.29-1.42$

not determined

$1.31-1.71$

$1.31-1.65$
$\underline{\mathrm{K}_{\mathrm{FeSi}}}$

$$
\begin{gathered}
1.12-1.57 \\
1.09-1.61 \\
\text { not determined }
\end{gathered}
$$

$1.22-1.59$

not determined

not determined 
In general, the higher values of $\mathrm{k}_{\mathrm{MgSi}}$ and lower values of $\mathrm{k}_{\mathrm{FeSi}}$ represent the larger particles. The overall mean values for the factors were $\mathrm{k}_{\mathrm{MgSi}}=1.41$ and $\mathrm{k}_{\mathrm{FeSi}}=1.48$. These values include fundamental constants such as fluorescent yield, the influence of microscope parameters such as the accelerating voltage influence on ionization crosssection, and $x$-ray detector parameters such as beryllium window thickness, and so will vary from one experimenter and apparatus to another.

Since practical identification of mineral particles in the microscope normally involves many particles, it is necessary to restrict the analyzing time per particle. As an apparently practical compromise, we chose to count until the silicon peak reached 1000 full scale (at which point its integrated area was 5500-6000 counts). This required times varying from less than 20 seconds for the larger particles to more than 100 seconds for the smaller ones.

For a series of ten such measurements, all on a single particle of each type (each approximately $0.5 \mu \mathrm{m}$ in particle or fiber diameter), we then integrated the $\mathrm{Mg}$, $\mathrm{Si}$, and $\mathrm{Fe}$ peaks and divided to obtain ratios. Table 3 shows the range of variation due to the statistics of counting, and figure 3 shows a representative spectrum.

Table 3. Range of intensity ratios for ten measurements on same particle.

\begin{tabular}{lcc}
\multicolumn{1}{c}{$\frac{\mathrm{Mg} / \mathrm{Si} \mathrm{i}}{1}$} & $\underline{\mathrm{Fe} / \mathrm{Si}}$ \\
Amosite & $0.023-0.181$ & $0.917-1.008$ \\
Anthophyllite & $0.410-0.489$ & $0.132-0.202$ \\
Chrysotile & $0.946-1.036$ & $0.021-0.104$ \\
Crocidolite & $0.010-0.129$ & $0.822-0.916$ \\
Talc & $0.402-0.511$ & $0.006-0.051$ \\
Tremolite & $0.307-0.413$ & $0.018-0.111$
\end{tabular}

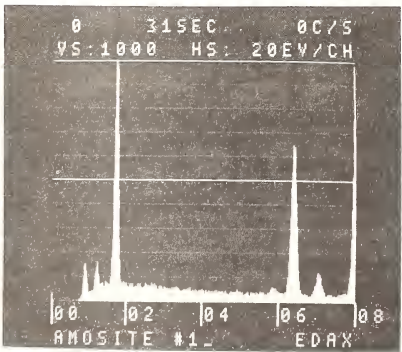

Figure 3. Repetitive short time measurement on fiber of amosite, showing poor peak statistics.

Figure 4 shows these intensity ratio ranges graphically, and figure 5 shows the same ranges enlarged by the error due to assuming the $k_{x S i}$ factor is constant. It is clear that while many of the minerals can be readily distinguished, not all can. For amosite and crocidolite, some additional means of identification such as the presence of other elements, or electron diffraction must be added to the simple comparison of intensity ratios of the major elements. Identification of more (preferably al1) of the elements present in a mineral can in principle end in positive identification of the mineral. For example, the 
sodium in crocidolite and manganese in amosite are distinctive. However, in this case the - peak of copper (from the sample grid) obscures the presence of any sodium peak, and in any zase both peaks are smaller than the principal elements already used, so that longer analysis times would be needed to obtain statistically useful numbers. It is not practical to use a longer time to reduce the statistical spread, as the overlap would persist.

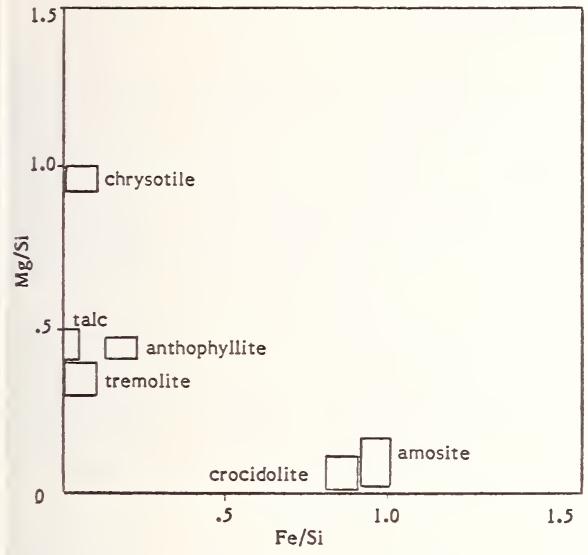

Figure 4. Plot of intensity ratios for minerals (see Table 3).

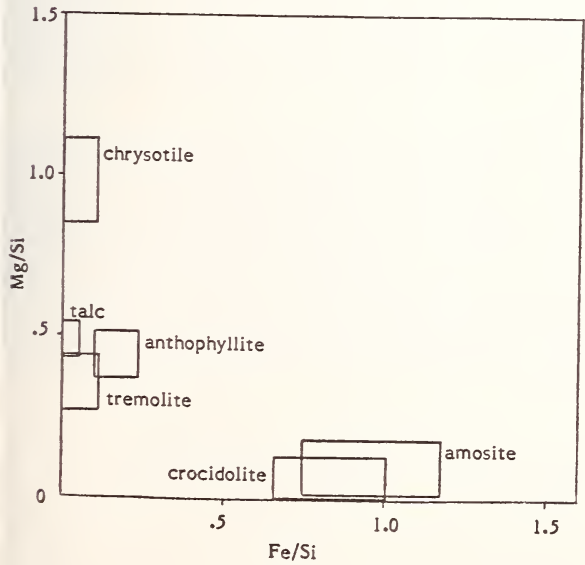

Figure 5. Plot of total range of intensity ratios, including variation in $k_{x S i}$ due to particle size, geometry and matrix. 
Most of the error introduced by neglecting the effect of particle size on the $K_{x S i}$ factor can be eliminated by correcting for absorption, as shown by Goldstein [7]. The factors measured on standards can be converted to the "infinitely thin" case by dividing them by

$$
\left.e^{-[(\mu / \rho)} S_{i}^{-(\mu / \rho)} x\right] \rho t / 2
$$

where $(\mu / \rho)$ is determined for the matrix as $\Sigma C_{i}(\mu / \rho)_{j}$ from each individual element's mass absorption coefficient, $\rho$ is the density, $t$ is the approximate size of the particle, and $C_{i}$ are the concentrations of the elements present. Then they should be constant independent of size. When unknowns are measured, the factor can be adjusted by the same method, except that while the size can be determined adequately from the TEM image, the concentrations are not known. This is awkward, since it means some iterative calculation is needed. Furthermore, only the concentration ratios are being determined, not the absolute concentrations. Also, the density can only be guessed at. For these asbestos materials, normalizing the elemental ratios of $\mathrm{Mg}, \mathrm{Si}$, and $\mathrm{Fe}$ to arbitrarily total to 50 percent gives adequate results. However, in other quite different materials this could lead to significant errors.

Individual correction of the $\mathrm{k}_{x \mathrm{Si}}$ factors for particle size, and conversion to concentration, is only useful for spectra having good counting statistics, and so is too time consuming for routine application to large numbers of analyses. Nevertheless, the use of fully corrected results (i.e. the conversion of spectral data to oxide chemistry with total propagated errors due to statistics) is the only reliable way to obtain meaningful concentrations. This is even more true if different investigators' results are to be compared, since the $k_{x S i}$ factors are dependent on the accelerating voltage used. The calculation can also include atomic ratios of the cations present, which may be useful for phase identification as well.

An additional caution is that since analysis of samples of this type requires collecting data from large numbers of particles or fibers, the problem of total counting calculation time becoming large to obtain more accurate results can be prohibitive. On the other hand, one should not assume that the errors described in the simple use of intensity ratio matching will tend to average out when large numbers of fibers are analyzed. This does not happen, as the errors are generally likely to be strongly biased, and not random. Confused identification is most likely to result when there are mixtures of mineral types present, a frequent occurrence in samples of real interest.

The use of x-ray analysis, particularly with interpretation based on simple elemental ratios, is certainly not adequate for identification of asbestos minerals with the high confidence needed in view of the health and legal concerns regarding these minerals. If the $x$-ray results are combined with morphology (e.g., fibrous appearance) and, when they can be obtained, electron diffraction patterns, then fairly conclusive identification can be made [8]. Unfortunately, this combination requires a skilled and knowledgeable microscopist/analyst, and furthermore is too time-consuming for application to large numbers of particles. Hence, there will probably continue to be some use of the simple ratio method, which may be permissible if the potential errors and resulting confusions in identification are first studied for the particular case of interest. In general, the experimenter should be very careful in applying this fast and simple method. It is essential to first consider all probable overlaps of identification with other minerals which might be present.

The author is particularly grateful to Dr. F. D. Pooley for advice and comments. 
(1] Beaman, D. R. and File, D. M., Quantitative determination of asbestos fiber concentrations, Anal. Chem., 48, 101-110 (1976).

2] Pooley, F. D., The use of an analytical electron microscope in the analysis of mineral dusts, Phil. Trans. R. Soc. Lond. A., 286, 625-638 (1977).

3] Pooley, F. D., The identification of asbestos dust with an electron microscope microprobe analyzer, Norelco Reporter, Special Issue, 5-9 (1976).

[4] Cliff, G. and Lorimer, G. W., Quantitative analysis of thin foils using EMMA 4 - the ratio technique, Proc. Fifth Europ, Congr. on Electron Microsc. Manchester, p. 140-141, London; Inst. of Physics.

[5] Russ, J. C., The direct element ratio model for quantitative analysis of thin sections, Microprobe Analysis as Applied to Cells and Tissues, Hall, T., Echlin, P., and Kaufmann, R., 269-276, (1974).

[6] Pooley, F. D., private communication.

[7] Goldstein, J. I., Lorimer, V. W., and Cliff, G., Quantitative x-ray analysis of thin films in the electron microscope, MAS Proceedings, 11th Annual Conf., Aug. 1976, p. $25 A-C$.

[8] Chatfield, E. J., Identification and measurement of asbestos fibers by electron microscopy, Asbestos, February and March 1978. 
National Bureau of Standards Special Publication 533. This Publication Originated from a Special Session on Particle Analysis, 13th Annual Conference of the Microbeam Analysis Society, Ann Arbor, MI, June 22, 1978. (Issued Apri1 1980)

\title{
QUANTITATIVE ANALYSIS OF SMALL PARTICLES USING WAVELENGTH AND ENERGY DISPERSIVE SYSTEMS IN AN ELECTRON BEAM INSTRUMENT
}

\author{
John Gavrilovic \\ Walter C. McCrone Associates, Inc. \\ Chicago, I1linois 60616
}

Microparticles in sizes down to $1-2 \mu \mathrm{m}$ can be quantitatively analyzed in electron beam instruments using the standard ZAF correction procedure for solid (bulk) and infinitely large samples. The accuracy of such quantitative analyses is limited primarily by the particle size and subsequent loss of x-ray intensities which, in turn, increase experimental errors. Such errors cannot be effectively compensated by small particle corrections since they are mostly random and are caused by instrument instability and counting errors. Ratios of $x$-ray intensities for particles below $1 \mu \mathrm{m}$ and particularly below $0.3 \mu \mathrm{m}$ are severely affected by particle size. The relative change in measured x-ray intensities on very small particles as compared to bulk samples is caused primarily by the decrease in x-ray absorption due to the shorter path within the sample and is significantly higher than the experimental errors incurred during the analysis. Special correction procedures that have been developed or are currently being developed can be applied to improve the accuracy of the analysis of such particles $[1,2,3]^{1}$.

The total intensity of $x$-rays from small particles (below 6-10 $\mu \mathrm{m}$ ) falls rapidly with particle size, as can be seen in figures 1 and 2. Particles larger than 6-12 um have total $x$-ray intensities equal to that of a bulk sample. The major cause of the significant drop in x-ray intensity for particles larger than $10 \mu \mathrm{m}$, observed by some researchers, is the result of local changes both in the incident angle of the electron beam and in $x$-ray takeoff angle, both of which depend on particle shape [4]. The optimum method for analysis of small particles therefore appears to be to collect $x$-rays from the small particle while it is being rastered by the electron beam. Such a raster normally also covers a certain portion of the substrate in order to incorporate the whole projected area of the small particle. To compensate for loss of x-ray intensity during rastering of the substrate one can use the following correction factor:

$$
I_{0}=I_{1} \times \frac{S_{r}}{S_{p}},
$$

where $I_{0}$ is the corrected intensity from the element, $I_{7}$ the measured intensity, $S_{r}$ the total rastered area, and $S_{p}$ the projected area of the small particle. With this method the stability of the measurement is significantly increased and analysis of particles down to approximately $1 \mu \mathrm{m}$ becomes routine. Even particles smaller than $1 \mu \mathrm{m}$ down to $0.1 \mu \mathrm{m}$ can be analyzed semiquantitatively with a relative accuracy of 20-40 percent without further correction except for the ratio of rastered to projected area.

${ }^{1}$ Figures in brackets indicate the literature references at the end of this paper. 


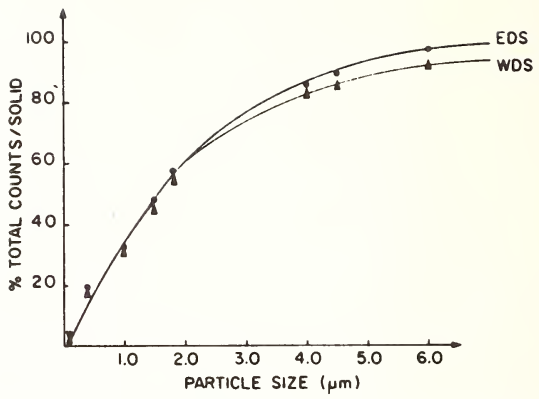

Figure 1. Size distribution versus total intensity of major elements for K-489 glass particles.

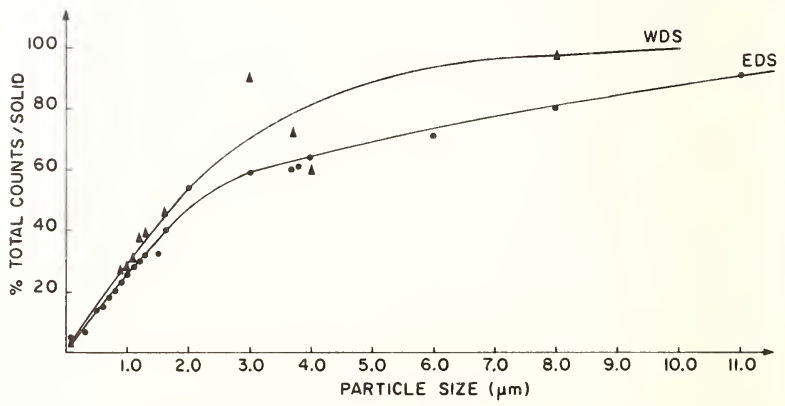

Figure 2. Size distribution versus total intensity of major elements for K-230 glass particles. 
Both wavelength and energy dispersive systems can be used for quantitative analysis of mall particles without corrections provided suitable bulk standards of similar composition ire available. Low concentration elements (less than $1 \%$ ) in small particles should be inalyzed with wavelength dispersive systems because of the excessively high background nherent in the energy dispersive systems.

Figures $3,4,5$, and 6 show experimental results obtained during analysis of microarticles of sizes $0.1 \mu \mathrm{m}$ and larger mounted on a polished beryllium substrate. These ifcroparticles were obtained by grinding two types of glass standards produced by the lational Bureau of Standards for microbeam analysis. As you can see from these diagrams, he calculated composition for small particles, down to approximately $2 \mu \mathrm{m}$ is the same as or bulk samples of glass without any corrections. Below approximately $1.5 \mu \mathrm{m}$ the ratio of lements in the small particles drastically changes due to the increase in the ratio of low energy to high energy $x$-rays. This is a result of reduction in self-absorption in the imaller sample. Such changes are frequently masked by large relative errors common in small article analysis [5]. The wavelength dispersive system gives a lower background and higher ensitivity for trace elements, but the associated experimental errors are generally larger ecause of the more severe $x$-ray geometry requirements.
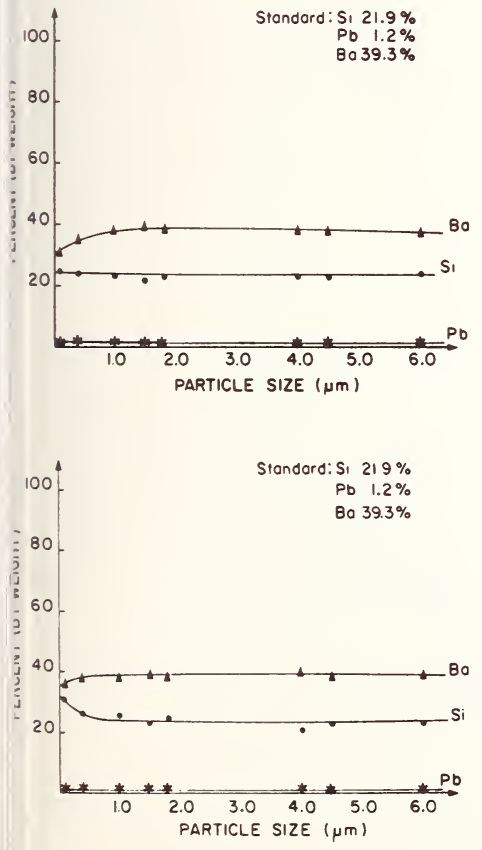

Figure 3. Percent by weight of major elements versus size as determined by EDS for K-489 glass particles.
Figure 4. Percent by weight of major elements versus size as determined by WDS for K-489 glass particles. 

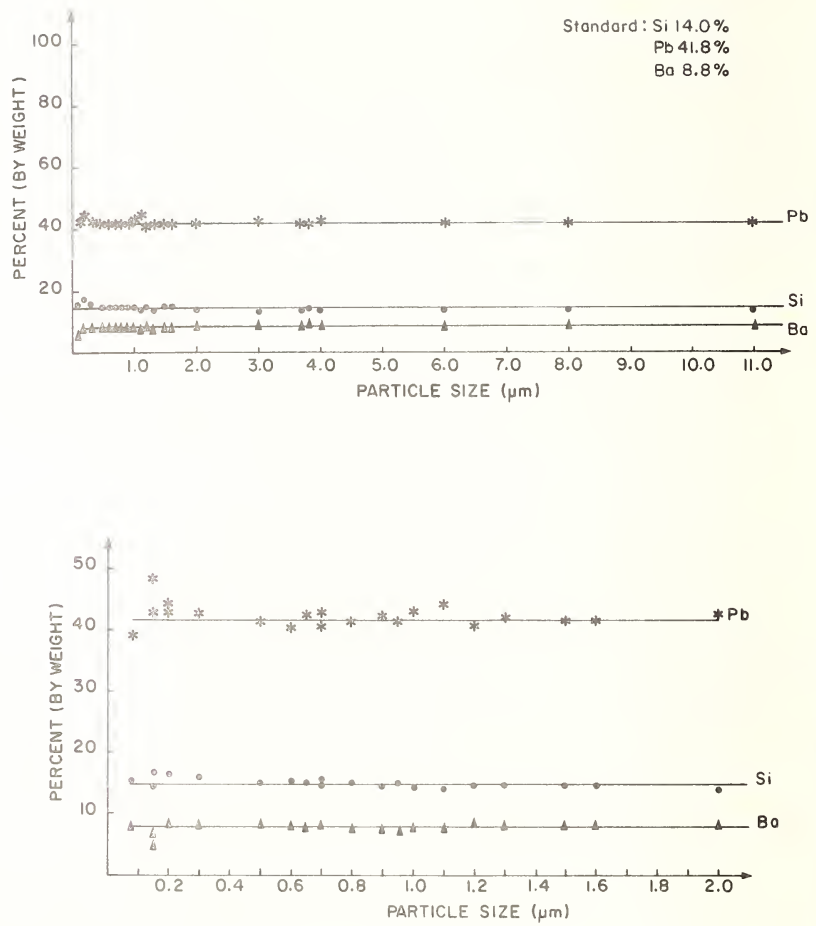

Figure 5. Percent by weight of major elements versus size as determined by EDS for K-230 glass particles (bottom curves - expanded scale). 

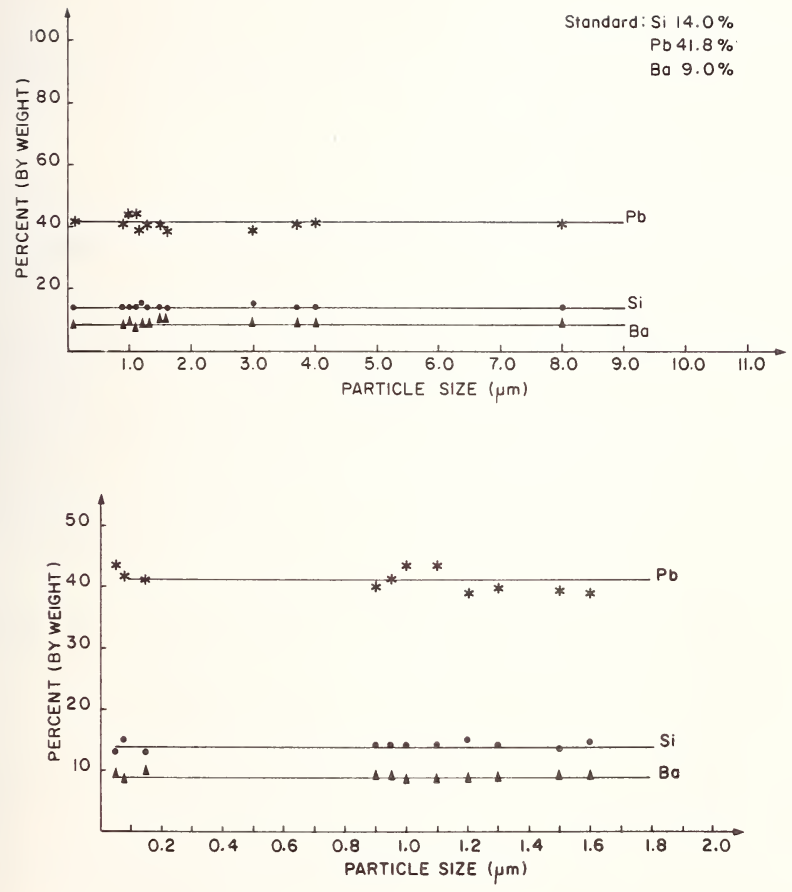

Figure 6. Percent by weight of major elements versus size as determined by WDS for $\mathrm{K}-230$ glass particles (bottom curves - expanded scale. 
A similar set of curves, Figures 7 and 8 , show an even more remarkable similarity in composition of the analyzed microparticles and a bulk glass standard $\mathrm{K}-249$, down to $0.2 \mu \mathrm{m}$ size. Below that size, experimental errors due to low count rates virtually mask the particle size effect.

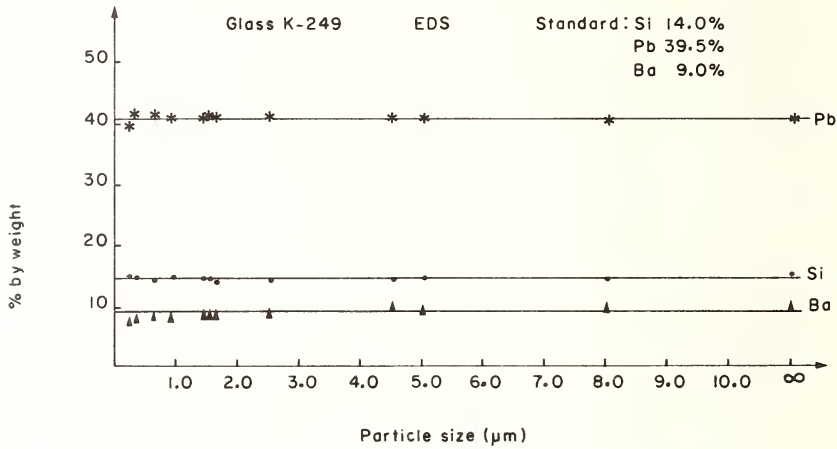

Figure 7. Percent by weight of major elements versus size as determined by EDS for $\mathrm{K}-249$ glass particles.

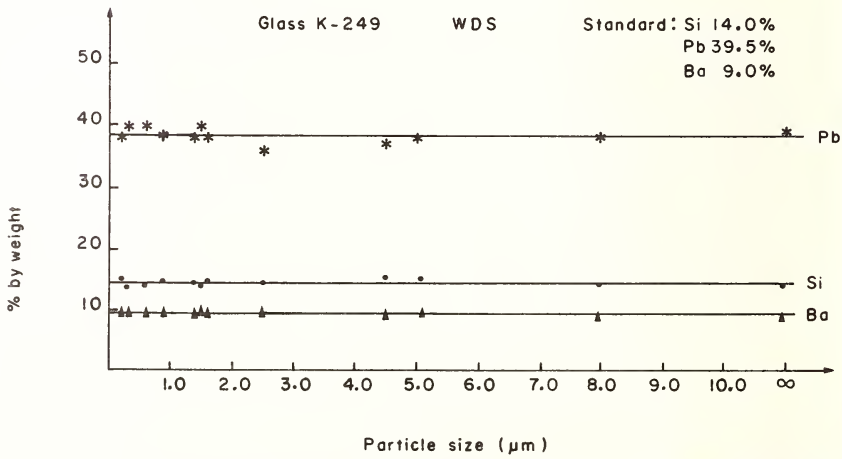

Figure 8. Percent by weight of major elements versus size as determined by WDS for $\mathrm{K}-249 \mathrm{glass}$ particles. 
Table 1 illustrates the results of an EDS analysis of 30 small particles ranging in size from 0.1 to $11 \mu \mathrm{m}$, including the bulk (polished) glass standard $\mathrm{k}-230$. These results were obtained on an ARL electron microprobe, EMX-SM type, at $10 \mathrm{nA}$ specimen current on beryllium and $20 \mathrm{kV}$ accelerating voltage with an electron beam diameter of $0.1 \mu \mathrm{m}$. The probe is equipped with four wavelength-dispersive spectrometers and a high resolution ORTEC energy dispersive detector along with a Sargent-Welch vertical turbomolecular pump which supplies $1 \times 10^{-6}$ torr contamination-free vacuum.

With the steady increase in the precision and accuracy of electron beam analysis of small particles, special correction methods will be used more frequently. The development of a relatively simple, reliable, computerized correction procedure will then be of great help to all small particle analysts.

\section{References}

[1] Armstrong, J. T., and Buseck, P. R., The Minimization of Size and Geometric Effects in the Quantitative Analysis of Microparticles with Electron Beam Instruments, Proceedings of the Tenth Annual Conference of Microbeam Analysis Society.

[2] Armstrong, J. T., and Buseck, P. R., Quantitative Chemical Analysis of Individual Microparticles Using the Electron Microprobe, Anal. Chem., 47, 2178-2192 (1975).

[3] Small, J. A., Heinrich, K. F. J., et al., Progress in Quantitation of Single Particle Analysis with the Electron Probe, Proceedings of Fifteenth Annual Conference of Microbeam Analysis Society, Ann Arbor, MI (1978).

[4] Bayard, M., Microprobe Analysis, Andersen, C. A. ed., Wiley-Interscience, New York, NY, (1973) pp. 323-348.

[5] Gavrilovic, J., Common Errors in Subnanogram Particle X-ray Analysis, Microscope, 22, 221-227 (1974). 


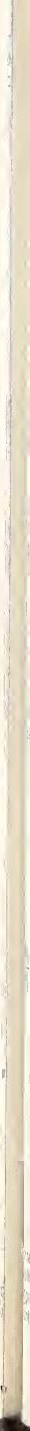


National Bureau of Standards Special Publication 533. This Publication Originated from a Special Session on Particle Analysis, 13th Annual Conference of the Microbeam Analysis Society, Ann Arbor, MI, June 22, 1978. (Issued Apri1 1980)

PROCEDURE FOR THE QUANTITATIVE ANALYSIS OF SINGLE PARTICLES WITH THE ELECTRON PROBE

J. A. Sma11, K. F. J. Heinrich, D. E. Newbury, R. L. Myklebust, and C. E. Fiori

\author{
Center for Analytical Chemistry \\ National Bureau of Standards \\ Washington, D. C. 20234
}

\title{
Introduction
}

In recent years, one of the major areas of interest in the field of electron probe analysis has been the elemental characterization of individual particles. In order to develop a quantitative analysis procedure for particles, there are two major effects peculiar to particulate analysis which must be taken into account.

The first effect arises from the finite size (mass) of a particle. This mass effect is important when the excitation volume of the primary electron beam is larger than the volume of the particle to be analyzed. In this situation, the mass of material participating in $x$-ray generation is less in the particle than it is in a thick specimen since the beam electrons may escape through the sides and bottom of the particle.

This effect also results in a reduction of $x$-ray intensities from the particles relative to the intensities from bulk materials; the deviation from bulk behavior increases in magnitude with decreasing particle size. The mass effect can be demonstrated by comparing the $x$-ray emissions from a particle to the emissions from a bulk target of the same composition. The effect is most evident when the comparison is done for a hard energy $x$-ray 1 ine which has minimal absorption. Such a case is shown in figure 1 by the decrease in the $\mathrm{Ba} L \alpha$ intensity from the particles relative to the bulk material for particles smaller than $3 \mu \mathrm{m}$ in diameter.

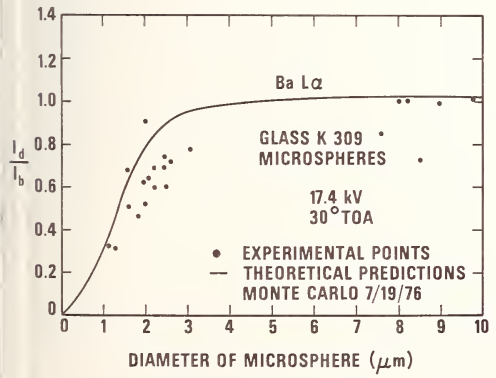

Figure 1. Ba L $\alpha$-ray intensity for glass spheres ratioed to bulk intensity and plotted as a function of sphere diameter. $I_{d}=$ sphere intensity, $I_{b}=$ bulk intensity and $T O A=$ take-of $f$-angle.

The second major effect is the result of absorption and is important when the average $x$-ray absorption path length for a particle is different from the average path length for a bulk material. The largest deviations from bulk occur when there is high absorption as is typically observed for low energy $x$-ray lines. Such a situation is common in many samples containing elements such as aluminum and silicon. The difference in average path length 
can result in widely different values of $x$-ray intensities between particle and bulk. For the situation shown in figure 2, the path length $A-B$ in the particle is less than the path length $\mathrm{A}-\mathrm{C}$ in the bulk. The reduced average path length results in a greater $\mathrm{x}$-ray emission from the particle compared to bulk, despite the opposing action of the particle mass effect. The absorption effect is demonstrated in figure 3 where the silicon intensity ratio between particle and bulk is above unity for spheres greater than $2.0 \mu \mathrm{m}$ in diameter.

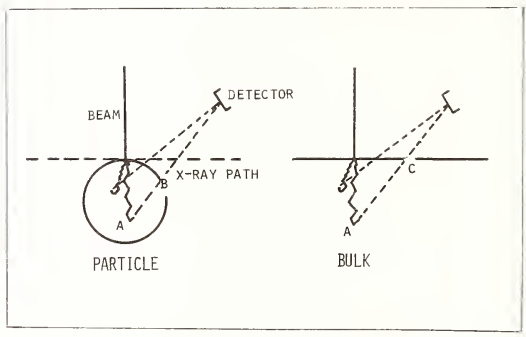

Figure 2. Diagram of particle absorption effect.

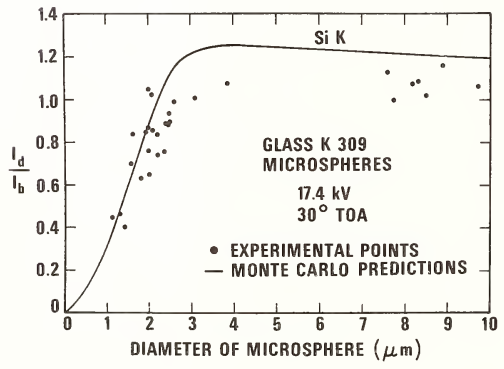

Figure 3. Si K x-ray intensity for glass spheres ratioed to bulk intensity and plotted as a function of sphere diameter. $I_{d}=$ sphere intensity, $I_{b}=b u l k$ intensity and TOA=take-off-angle.

Various analytical techniques have been developed for the analysis of particles. These procedures range in complexity from the simple scaling of the peak intensities obtained by conventional analysis programs such as the procedure used by Gavrilovic [1] $]^{1}$ to the extensive treatment based on shape determination proposed by Armstrong [2]. The main problems with these existing techniques are that the complex procedures require a large computer and extensive analyst training, while the simple scaling procedure is limited to a small number of particle compositions for which the particle effects are minimized.

${ }^{1}$ Figures in brackets indicate the literature references at the end of this paper. 
At the 1978 Scanning Electron Microscopy meeting, a new approach to quantitative inalysis of particles based on peak-to-background intensity measurements was proposed ndependently by Small et al. for the determination of concentrations, and by Statham and awley for the determination of concentration ratios $[3,4]$. This new approach is based on he use of the ratio between the characteristic $x$-ray intensities and the continuous $x$-ray ntensities of the same energies (peak-to-background ratios) to eliminate the major particle ffects. It is a modification of the method proposed by Hall to correct for the excitation 07 me in thin films [5]. In this paper, the current status of the analytical procedure eing developed at NBS for particle analysis will be described. In addition, the results btained from the analysis of glass and mineral particles with this procedure will be ompared to the results obtained from conventional microprobe analys is procedures.

\section{Experimental Techniques}

In order to test the particle analysis scheme, it was necessary to obtain particles of nown composition. For this purpose, glass particles were made by crushing bulk glass from he set of NBS Research Material Glasses [6]. Mineral particles of talc and $\mathrm{FeS}_{2}$ were also btained for the study. Al1 particles were suspended in ethanol and mounted on a $20 \mathrm{~nm}$ arbon film which was supported by a beryllium microscope grid. The samples were coated by acuum evaporation with approximately $10 \mathrm{~nm}$ of carbon before analysis.

The experimental measurements were made with an electron microprobe analyzer equipped ith a lithium-drifted silicon x-ray detector. The beam energy used for the measurements as $20 \mathrm{keV}$, the take-off angle was 40 degrees, and the beam current was $1 \times 10^{-9} \mathrm{~A}$. The articles were analyzed by scanning the electron beam over an area larger than the crossectional areu of the particle in order to minimize beam positioning effects. The analytcal conditions were the same for the mineral particles, but the beam energy was $10 \mathrm{keV}$ for alc and $15 \mathrm{keV}$ for $\mathrm{FeS}_{2}$.

\section{Calculation of the Peak-to-Background Ratios}

The peak-to-background ratios, $R$, are calculated from equation (1) in which $I_{E}$ equals he experimental intensity within a region of interest of photon energies for a characterstic line, $I_{B k g}$ equals the calculated background intensity, obtained by the data reduction rocedure, FRAME C [7], for the same region of interest:

$$
R=\frac{\left(I_{E}-I_{B k g}\right)}{I_{B k g}}
$$

he continuum from the carbon $f i 1 \mathrm{~m}$ is ignored and the particle spectrum is assumed to have continuum which originates from only the analyzed particle. This assumption is reasonble since the intensity from the carbon film is small in most cases as shown by the plots $\mathrm{n}$ figure 4 , which are the superimposed spectra from an $8 \mu \mathrm{m}$ particle and the carbon film. 


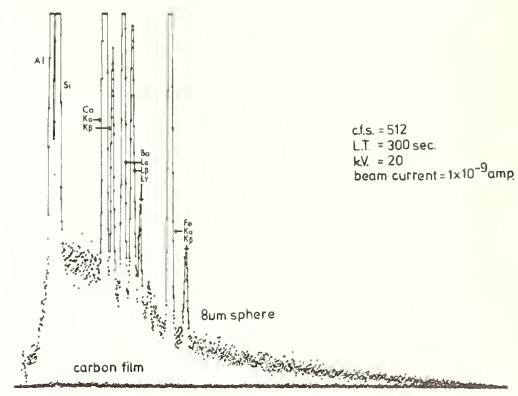

Figure 4. Spectra of $8 \mu \mathrm{m}$ particle and carbon $\mathrm{film}$.

\section{Calculation of Particle Continuum}

The continuum for a given particle is fit by a computer with equation (2) which is Lifshin's modification of Kramers' equation $[8,9]$. It was originally derived for bulk specimens and is being used to calculate a first approximation for the particle continuum.

$$
I_{E}=\left[A \cdot\left(E_{0}-E\right)+B \cdot\left(E_{0}-E\right)^{2}\right] \cdot 1 / E f(x) \cdot P_{E}
$$

$I_{E}$ equals the intensity of the continuum at energy $E, E_{0}$ equals the accelerating voltage, $A$ and $B$ are empirical coefficients, $f(x)$ is the absorption term for a bulk specimen, and $P_{E}$ is the detector efficiency. Two experimental regions of interest are used to determine the coefficients $A$ and $B$. The equation is then used to calculate the continuum as a function of energy.

Figure 5 shows an actual spectrum of a particle $10 \mu \mathrm{m}$ in diameter superimposed on the calculated background determined by equation 2. One of the continuum regions selected for the fit was taken between the $\mathrm{Si} K$ and $\mathrm{Ca} \mathrm{K \alpha}$ peaks, and the other at an energy slightly greater than the $\mathrm{Fe} K \beta$ peak. The fit is in good agreement with the experimental continuum over the entire energy range.

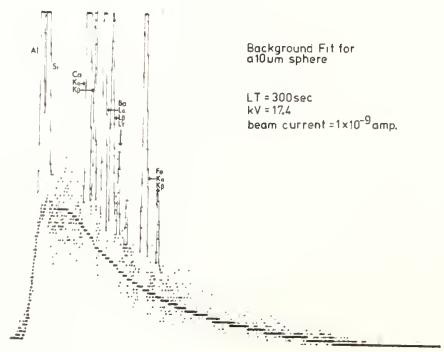

Figure 5. Spectrum showing a $10 \mu \mathrm{m}$ particle and the background fit. 
Unlike the procedure being developed by Statham in which the results are expressed as concentration ratios, the method under development at NBS expresses results as elemental concentrations.

Equation (3) is the foundation for the particle procedure and resulted from the experimental observation that the emitted peak-to-background ratios from particles are, to a first approximation, equivalent to the peak-to-background ratios from a bulk sample of the same composition [3]. Although the physical mechanisms of generation of characteristic and bremsstrahlung $x$-rays are different, to a first approximation the two radiations for a given energy are formed over nearly the same region of the sample. Therefore, the ratio of the peak intensity to the background intensity of the same energy can be used to normalize out the effects of absorption and mass.

$$
(\mathrm{P} / \mathrm{B})_{\text {particle }}=(\mathrm{P} / \mathrm{B})_{\text {bulk }}
$$

Rearranging equation (3), the peak intensities for the particle can be used to calculate the values appropriate to the intensity from a hypothetical bulk material of the same composition, $p *$, equation (4).

$$
P^{*} \equiv P_{\text {Bulk }}=\frac{P_{\text {Particle }}{ }^{B_{\text {Bulk }}}}{{ }_{\text {Particle }}}
$$

The values of the intensities, $p^{*}$ 's, can then be used to form $x$-ray intensity ratios $p^{*} / P_{\text {standard }}$, which would then be used as input for a conventional analysis procedure such as FRAME C. In practice, for a typical particle of unknown composition, bulk material with the same composition will not be available to measure the values of B (bulk). These values, however, can be approximately calculated from the continuum of a standard and from the first estimate of composition obtained from the hyperbolic iteration loop in the conventional FRAME $C$ program. These values of ${ }^{B}(B u l k)$ can then be used in equation (4) to obtain the first estimates of $p^{*}$ 's. A set of intensity ratios for a bulk sample of the same composition as the particle is then calculated from equation (5) and used as input for the ZAF routine. The set of concentrations from each iteration is used to calculate new values of ${ }^{B}$ (Bulk) and the sequence is repeated until convergence (in practice 5 iterations). A block diagram for the particle procedure is shown in figure 6 .

$$
k=p^{*} \text { particle } / \mathrm{P} \text { standard }
$$




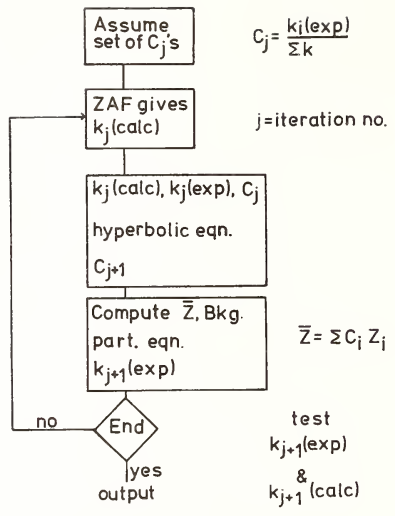

Figure 6. Flow chart showing the interface of the particle corrections with a ZAF analysis scheme.

Equation (6), which derives the intensity ${ }^{B}$ (Bulk) from that of the continuum on a standard, B(Std), uses the atomic number dependence of Kramers' law as modified by Green [10].

$$
B_{\text {Bulk }}=\frac{\bar{z}_{\text {Bulk }}}{\overline{\bar{z}}_{\text {Std }}} \cdot B_{\text {Std }} \cdot \frac{f_{\chi(B u l k)}}{f_{\chi(S t d)}} \cdot \frac{F\left(\bar{z}_{B u l k}\right)}{F\left(\bar{z}_{S t d}\right)}
$$

The subscript "Bulk" refers to the values which are calculated from the current estimate of the composition as determined by the analysis procedure, and the subscript "Std" refers to values for the standard. The $\overline{Z^{\prime}} s$ are the average atomic numbers, the $f_{x}$ 's are the absorption terms, and the $F(\bar{Z})^{\prime} s$ are the values of Green's correction to Kramers' constant. The values for $F(\bar{Z})$ were determined by a computer fit to the experimental curve in Green's thesis [11].

Results from the analysis with Kramers' law of NBS glass K-309 particles are shown in Table 1. Both particles were irregularly shaped and are shown in figures 7a and 7b. Glass K-411 (see Table 2) was used as the standard for $\mathrm{Si}, \mathrm{Ca}$, and $\mathrm{Fe}$, and bulk glass $\mathrm{K}-309$ was used as the standard for $\mathrm{Al}$ and $\mathrm{Ba}$. The results from the particle program are substantially closer to the nominal values than the results from FRAME $C$. The relative errors for the particle results are all under 10 percent except for $\mathrm{Si}$, which is 11 percent. In comparison, the results from FRAME $C$ have relative errors on the order of 65 percent. 
Table 1. Analysis of K-309 Particles

\begin{tabular}{|c|c|c|c|c|c|}
\hline Element & $\begin{array}{c}\text { True } \\
\text { Composition }^{a}\end{array}$ & \multicolumn{2}{|c|}{$\begin{array}{cc}\text { Particle Frame } \\
7 \mathrm{a} & 7 \mathrm{~b} \\
\end{array}$} & $\begin{array}{r}\text { Regu } \\
7 \mathrm{a}\end{array}$ & $\begin{array}{c}\text { Frame } \\
7 \mathrm{~b}\end{array}$ \\
\hline & (wt. \%) & \multicolumn{2}{|c|}{ (wt. \%) } & \multicolumn{2}{|c|}{ (wt. $\%$ ) } \\
\hline Al & 7.9 & 7.0 & 8.4 & 1.4 & 3.4 \\
\hline Si & 18.7 & 17.8 & 20.8 & 3.5 & 7.9 \\
\hline $\mathrm{Ca}$ & 10.7 & 9.9 & 10.1 & 2.0 & 3.9 \\
\hline $\mathrm{Ba}$ & 13.4 & 12.4 & 12.8 & 2.6 & 5.0 \\
\hline $\mathrm{Fe}$ & 10.5 & 9.3 & 10.4 & 2.1 & 4.3 \\
\hline
\end{tabular}

K-411 Glass, $\overline{\mathrm{Z}}-13$ was the standard for $\mathrm{Si}, \mathrm{Ca}, \mathrm{Fe}$.

K-309 Glass, $\overline{\mathrm{Z}}$-19 was the standard for $\mathrm{Al}$, Ba.

Beam energy was $20 \mathrm{keV}$.

True composition determined by electron probe analysis.
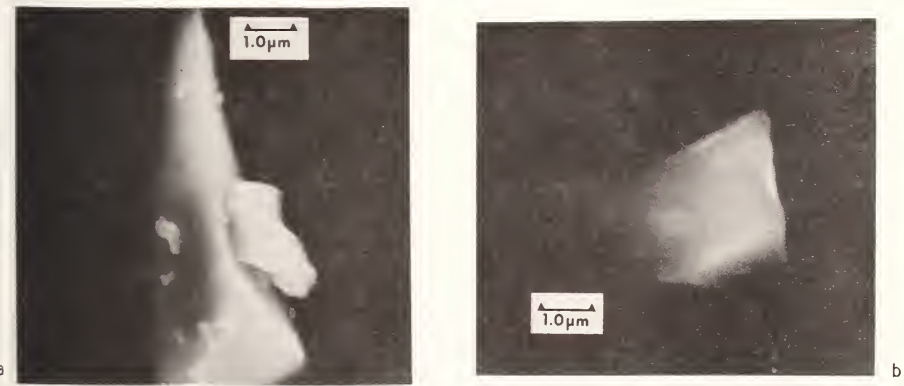

Figure 7 a and b. Micrographs of K-309 glass particles 1 and 2 .

Table 2. Composition of Glass K-411

\begin{tabular}{cc} 
E1ement & Wt. percent $^{\text {a }}$ \\
\cline { 2 - 2 } $\mathrm{Si}$ & 25.39 \\
$\mathrm{Fe}$ & 11.20 \\
$\mathrm{Mg}$ & 8.84 \\
$\mathrm{Ca}$ & 11.06 \\
$\mathrm{O}$ & 43.51
\end{tabular}

Determined by wet chemical analysis. 
The second method used to calculate the values of ${ }^{B}(B u l k)$ from a standard is given by equation (7), which was derived empirically at NBS. This equation takes into account an experimentally observed nonlinear relationship between generated continuum intensity and atomic number. The numerical form was determined by fitting a curve to the plot of the generated continuum for several pure elements vs. the atomic numbers.

$$
B_{\text {Bulk }}=\frac{{ }_{(s t d)} \times\left[(0.006 E+0.0642) \bar{Z}_{B u 1 k}^{n}-0.012 E-0.088\right]}{(0.006 E+0.0642) \bar{Z}_{(s t d)}^{n}-0.012 E-0.088}
$$

$\mathrm{E}$ is the energy in $\mathrm{keV}$ for which corresponding intensity is to be calculated, and $\mathrm{n}$ is a fitting parameter which is dependent on the beam energy. The values of $n$ which have been observed are 1.0 for $10 \mathrm{keV}, 0.64$ for $20 \mathrm{keV}$, and 0.44 for $25 \mathrm{keV}$. The functional dependence of $\mathrm{n}$ on beam energy is currently being studied.

The results of the analysis with this equation for talc particles are shown in Table 3. The calculated values for $A l$ and Mg compare favorably between the methods based on Kramers' equation and the NBS equation. The analyses by both methods are well within a 10 percent relative error, while the values from FRAME C are about 63 percent too low.

Table 3. Analysis of talc particles ${ }^{\mathrm{a}}$.

\begin{tabular}{|c|c|c|c|c|c|c|}
\hline & Z & Stoic. $^{b}$ & Frame C & $\begin{array}{l}\text { Eq. (6) } \\
\text { Kramers }\end{array}$ & $\begin{array}{c}\text { Eq. (7) } \\
\text { NBS }\end{array}$ & $\begin{array}{c}\text { Average } \\
\text { Dimension }\end{array}$ \\
\hline$\# 1$ & $\begin{array}{l}12 \\
14\end{array}$ & $\begin{array}{l}19.3 \\
29.8\end{array}$ & $\begin{array}{r}8.7 \\
13.4\end{array}$ & $\begin{array}{l}19.0 \\
31.2\end{array}$ & $\begin{array}{l}19.1 \\
30.1\end{array}$ & $3 \mu \mathrm{m}$ \\
\hline$\# 2$ & $\begin{array}{l}12 \\
14\end{array}$ & $\begin{array}{l}19.3 \\
29.8\end{array}$ & $\begin{array}{l}5.6 \\
8.5\end{array}$ & $\begin{array}{l}18.7 \\
30.7\end{array}$ & $\begin{array}{l}19.1 \\
29.8\end{array}$ & $2 \mu \mathrm{m}$ \\
\hline \#3 & $\begin{array}{l}12 \\
14\end{array}$ & $\begin{array}{r}19.3 \\
29.8\end{array}$ & $\begin{array}{l}16.6 \\
25.4\end{array}$ & $\begin{array}{l}17.9 \\
29.0\end{array}$ & $\begin{array}{l}17.5 \\
27.2\end{array}$ & $6 \mu \mathrm{m}$ \\
\hline
\end{tabular}

Beam energy was $10 \mathrm{keV}$.

$\mathrm{K}-411 \mathrm{Table} 2$ was used as the standard for $\mathrm{Mg}$ and $\mathrm{Si}$.

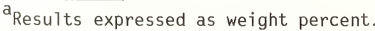

$\mathrm{b}_{\text {Stoichiometric concentration. }}$
}

A more dramatic effect can be seen in the analyses of $\mathrm{FeS}_{2}$ particles the results of which are reported in Table 4. These results show a decrease in the relative error for the analysis of $\mathrm{Fe}$ from 19 percent for the method based on Kramers' law to approximately 5 percent for the method based on the NBS equation. The relative error on the sulfur analyses is somewhat greater for particle \#2 when the NBS equation is used, but the value is still within six percent. 
Table 4. Analysis of $\mathrm{FeS}_{2}$ particles ${ }^{\mathrm{a}}$.

\begin{tabular}{|c|c|c|c|c|c|c|}
\hline & $\underline{z}$ & Stoic. $^{b}$ & Frame C & Kramers & NBS & $\begin{array}{c}\text { Average } \\
\text { Dimension }\end{array}$ \\
\hline$\# 1$ & $\begin{array}{l}16 \\
26\end{array}$ & $\begin{array}{l}53.4 \\
46.6\end{array}$ & $\begin{array}{l}31.3 \\
30.8\end{array}$ & $\begin{array}{l}53.2 \\
55.7\end{array}$ & $\begin{array}{l}53.5 \\
49.7\end{array}$ & $5 \mu \mathrm{m}$ \\
\hline$\# 1$ & $\begin{array}{l}16 \\
26\end{array}$ & $\begin{array}{l}53.4 \\
46.6\end{array}$ & $\begin{array}{l}46.0 \\
39.7\end{array}$ & $\begin{array}{l}53.2 \\
55.7\end{array}$ & $\begin{array}{l}56.2 \\
48.3\end{array}$ & $5 \mu \mathrm{m}$ \\
\hline
\end{tabular}

Beam energy was $15 \mathrm{keV}$.

ZnS and Fe were used as standards.

a Results expressed as weight percent.
$b_{\text {Stoichiometric concentration. }}$

At the present time, equation (7) is being tested in order to evaluate its applicaility to different detector and electron probe geometries. Equation (7) is also being lodified to improve the accuracy of predicting the continuous $x$-ray emission of a multilement system from a pure element standard.

\section{Additional Areas of Investigation}

In addition to the two major particle effects, there are several second-order correcions to the particle analysis scheme which need to be studied in more detail. The first $f$ these is manifested by an increase in the relative intensities of $x$-rays in bulk samples lue to characteristic and continuum fluorescence. The fluorescence-induced characteristic - ray production in bulk samples occurs over a range of distances greater than $10 \mu \mathrm{m}$ from he beam-sample interaction volume [3]. As a result, in micrometer-sized particles much of he fluorescence-induced $x$-ray intensity will be missing. This effect is most significant or heavy elements in a light matrix. For example, secondary fluorescence could cause eviations on the order of 5-10 percent for $\mathrm{Fe}$ in $\mathrm{K}-309$ particles relative to bulk targets s calculated by COR2 [12].

The second is a breakdown in the method used to fit the background on small particles. urrently the continuum for the particles is predicted by the modification of Kramers' quation mentioned earlier. This equation was derived for bulk specimens but is in good greement with the continuum for particles down to $10 \mu \mathrm{m}$ in size. For particles less than $0 \mu \mathrm{m}$, the predicted continuum is higher than the true value by about five percent. Efforts re underway to derive a more exact method for the prediction of the continuum emitted by ma11 particles.

Work is also being done with a Monte Carlo program in an effort to identify those conitions under which the correspondence of the peak-to-background ratio between particles nd bulk material of the same composition, equation (3), breaks down. Calculations which re presented in the paper by Newbury et al. elsewhere in this publication [13], show that here is a significant deviation in the peak-to-background ratios from particles as compared 0 bulk: (1) the anisotropy of the generation of the continuous $x$-radiation, and (2) diferences in the energy dependence of the cross-section for characteristic and continuous -radiation coupled with differences in the energy distribution of electrons backscattered rom bulk and particle targets. 
The proposed method for the quantitative analysis of particles is designed to obtain elemental concentrations rather than concentration ratios. The method involves minor alterations to a conventional microanalysis routine such as FRAME $C$, and can therefore be run in real-time (on-line) with a small computer. In addition, the method is, to a first approximation, independent of particle shape and requires no additional input other than that required for a conventional analysis.

The results from the analyses on synthetic and natural mineral particles are in good agreement with the known compositions and are substantially better than the results from conventional methods. In the near future additional corrections will be incorporated to account for the various second order effects.

\section{References}

[1] Gavrilovic, J., "Quantitative Analysis of Small Particles Using Wavelength and Energy Dispersive Systems in an Electron Beam Instrument," Proceedings of the 13th Annual Conference of the Microbeam Analysis Soc., Ann Arbor, MI., June 1978, paper \#60.

[2] Armstrong, J. T. and Buseck, P. R., "Quantitative Chemical Analysis of Individual Microparticles Using the Electron Microprobe: Theoretica1", Ana1. Chem., 47, 21782192 (1975).

[3] Sma11, J. A. et a1., "The Production and Characterization of Glass Fibers and Spheres for Microanalysis," SEM/1978/1, SRM Inc., AMF O'Hare, I1. 60666, pp. 445-454.

[4] Statham, P. and Pawley, J., "A New Method for Particle X-ray Micro-analysis Based on Peak Background Measurements," SEM/1978/1, SEM Inc., AMF O'Hare, I1. 60666, pp. 469478.

[5] Ha17, T., "Some Aspects of the Microprobe Analysis of Biological Specimens," in Quantitative Electron Probe Microanalysis, K. F. J. Heinrich, ed., U. S. Government Printing Office, Washington, D.C., 1968, pp. 269-299.

[6] Fiori, C. E. et a1., "An Overview of the Glass Standards Program for Microanalysis at the National Bureau of Standards," Proceedings of the 11th Annual Conference of the Microbeam Analys is Society, Miami Beach, F1., 1976, paper \#27.

[7] Myklebust, R. L. and Fiori, C. E., "FRAME C: A Compact Procedure for Quantitative Energy-Dispersive Electron Probe X-ray Analysis," Proceedings of the 12th Annual Conference of the Microbeam Analys is Society, Boston, MA., 1977, paper \#96.

[8] Kramers, H. A., "On the Theory of X-ray Absorption and of the Continuous X-ray Spectrum," Phi1. Mag., 48, 836 (1923).

[9] Lifshin, E., Proc. 9th Annual Conference of Microbeam Analysis Society, Ottawa, Canada, 1974, paper \#53.

[10] Green, M., Ph.D. Thesis, University of Cambridge, Chapter 4, 1962, pp. 60-75.

[11] Yakowitz, H. (Personal communication).

[12] Hénoc, J., Heinrich, K. F. J., and Myklebust, R. L., A Rigorous Correction Procedure for Quantitative Electron Probe Microanalysis (COR2), NBS Technical Note 769, U. S. Government Printing Office, Washington, D. C., 1973.

[13] Newbury, D. E., Myklebust, R. L., Heinrich, K. F. J., and Sma11, J. A., "Monte Car10 Electron Trajectory SimuTation - an Aid for Particle Analysis, this paper. 
National Bureau of Standards Special Publication 533. This Publication Originated from a Special Session on Particle Analysis, 13th Annual Conference of the Microbeam Analysis Society, Ann Arbor, MI, June 22, 1978. (Issued April 1980)

\section{MONTE CARLO ELECTRON TRAJECTORY SIMULATION - AN AID FOR PARTICLE ANALYSIS}

Dale E. Newbury, Robert L. Myklebust, Kurt F. J. Heinrich, and John A. Small

National Bureau of Standards

Center for Analytical Chemistry

Washington, D. C. 20234

\section{Introduction - Overview of Monte Carlo Simulation Techniques}

In many problems of interest in scanning electron microscopy and electron probe microanalysis, it is necessary to calculate the distribution of beam electrons within the sample, as well as the magnitude and distribution of the secondary signals (characteristic and continuum $\mathrm{x}$-rays, secondary electrons, cathodoluminescence, etc.) generated during interaction. The technique of Monte Carlo electron trajectory simulation has been widely applied to these oroblems [1] ${ }^{1}$. In a Monte Carlo electron trajectory calculation, the processes of elastic and inelastic scattering which take place during electron interaction are simulated through the use of mathematical models. The path of an individual electron is calculated through the solid in a stepwise manner as the electron undergoes scattering. The distance between scattering events, the scattering angles, and the rate of energy loss along the path are calculated from realistic physical models. Random numbers are used to distribute the choices for the parameters of these models over their respective ranges so as to accurately represent the relative probabilities for each process. Because of the large number of sossible choices for these parameters and the combinations thereof along a trajectory, the calculation of a single trajectory does not adequately describe the beam-specimen interaction, figure 1(a). To provide statistically valid results, a large number of trajectories nust be calculated. For trajectory plots which give a qualitative impression of the interaction volume, about one hundred individual trajectories must be calculated, figure 1(b), vhereas for quantitative results, one thousand to ten thousand trajectories are needed.

1. Types of Monte Carlo Simulations

The fundamental step of any Monte Carlo simulation which must be repeatedly calculated 1 1 ong the entire trajectory is illustrated in figure 2. With the electron at a point $P_{N}$, laving come from a previous point $\mathrm{P}_{\mathrm{N}-1}$, a scattering event is presumed to take place which changes the direction of the electron to bring it to a new point, $\mathrm{P}_{\mathrm{N}+\rceil}$. This scattering levent is characterized by an angle of scattering, $\phi$, which deviates the electron from its riginal path $\left(P_{N-7} \rightarrow P_{N}\right)$, an azimuthal scattering angle, $\psi$, which defines the plane within wich scattering occurs, and a distance between scattering events, the step length, S. hese parameters are used with an appropriate set of geometrical equations $[2,3]$ to produce 3 new set of coordinates $\left(X_{N+7}, Y_{N+7}, Z_{N+7}\right)$ and the process is repeated. Within this general framework, the various types of Monte Carlo simulations can be divided into three :lasses: (a) single scattering, (b) multiple scattering, and (c) hybrid.

\section{Single Scattering}

In a single scattering simulation, an appropriate scattering cross section is employed hich gives the probability for discrete scattering events. Since most of the angular leviations greater than $0.5^{\circ}$ occur as a result of elastic scattering, an elastic scattering ross section such as the total (relativistic) [4] Rutherford scattering cross section is employed:

Figures in brackets indicate the literature references at the end of this paper. 


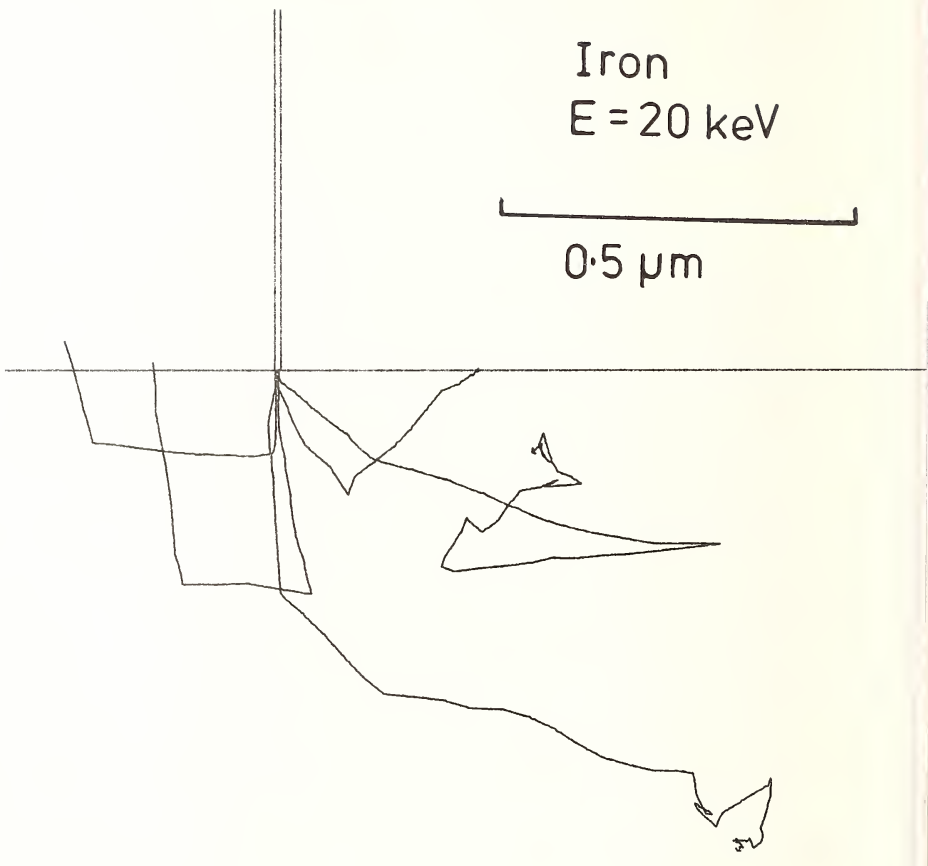

Figure la. Monte Carlo electron trajectory simulation. Target: iron; beam energy: $20 \mathrm{keV}$; beam diameter: $10 \mathrm{~nm}$; 5 trajectories.

40 


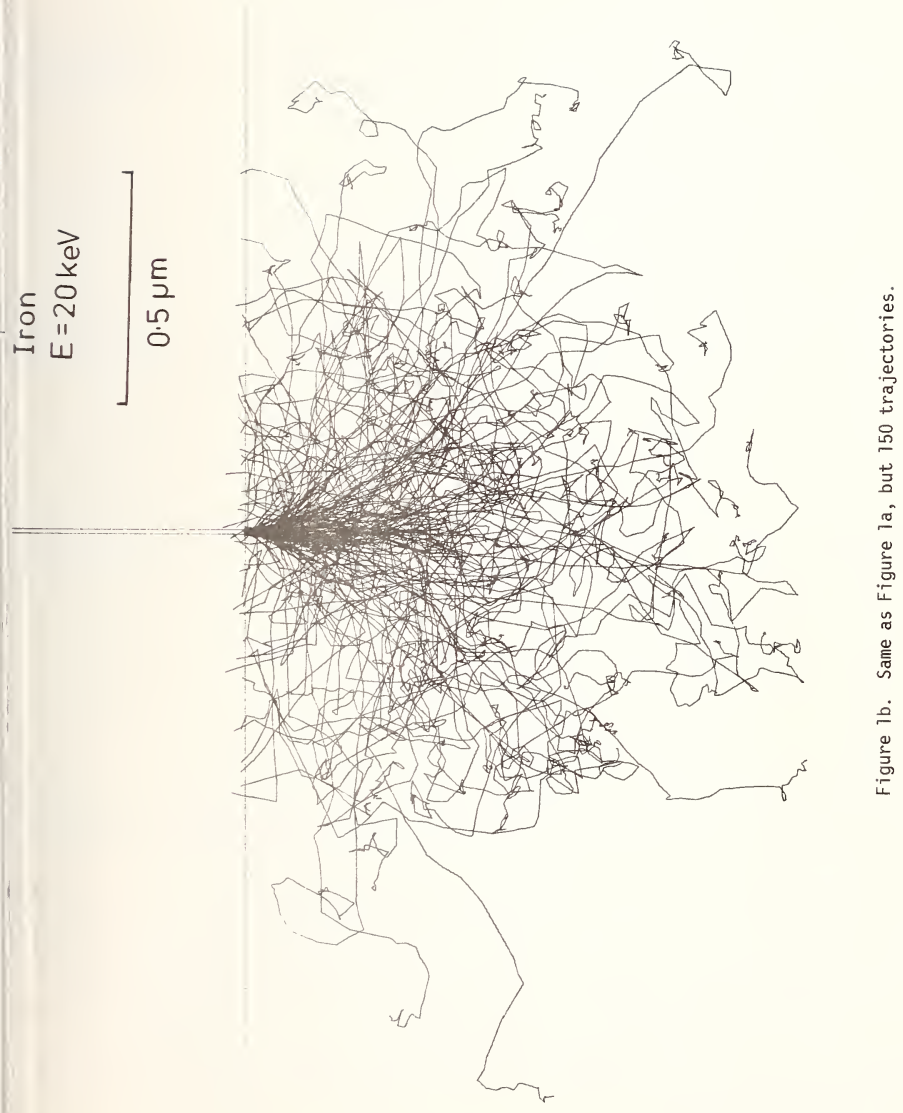




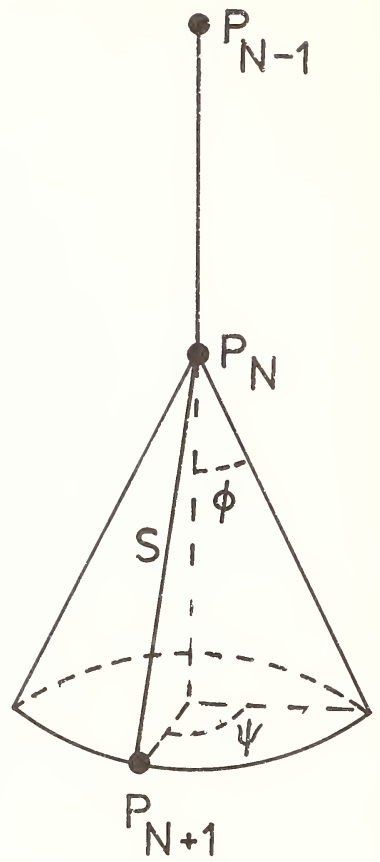

Figure 2. Elemental step in a Monte Carlo electron trajectory simulation. $\phi$ is the ellstic scattering angle; $S$ is the step length; $\psi$ is the azimuthal angle.

$$
\sigma_{E}=5.21 \times 10^{-21} \frac{z^{2}}{E^{2}} \frac{4 \pi}{\alpha(1+\alpha)}\left(\frac{E+m_{0} c^{2}}{E+2 m_{0} c^{2}}\right)^{2}
$$

where $\sigma_{E}$ is the total cross section $\left(\frac{\text { events }}{\text { electron }-\left(\frac{\text { atom }}{\mathrm{cm}^{2}}\right)}\right), Z$ is the atomic number, $E$ is the beam energy (neV), $m_{0}$ is the electron rest mass, $c$ is the speed of 1 light, $\left(m_{0} c^{2} \cong 511 \mathrm{keV}\right)$ and $\alpha$ is a screening factor [5]:

$$
\alpha=3.4 \times 10^{-3} z^{0.67} / E
$$

42 
which describes the effect of orbital charges on the electrostatic field of the nucleus. From the total cross section, a mean free path between scattering events, $\lambda$, can be calculated:

$$
\lambda=A /\left(N_{A} \rho \sigma_{E}\right)
$$

where $A$ is the atomic weight ( $\mathrm{g} / \mathrm{mole}$ ), $N_{A}$ is Avogadro's number (atoms/mole), and $\rho$ is the density $\left(\mathrm{g} / \mathrm{cm}^{3}\right)$. Since the value of $\lambda$ represents a mean free path, the particular value of the step length to be used in any step is taken from a statistical distribution about this imean [4]:

$$
S=-\lambda \log |R|
$$

The scattering angles are determined from the differential form of the cross section:

$$
\frac{d \sigma}{d \Omega}=5.21 \times 10^{-21} \frac{z^{2}}{E^{2}}\left(\frac{E+m_{0} c^{2}}{E+2 m_{0} c^{2}}\right)^{2} \frac{1}{\left(\sin ^{2} \frac{\phi}{2}+\alpha\right)^{2}}
$$

Given a probability distribution function, $f(x)$, where for example $f(x)$ can be the scattering cross section $\sigma(\theta)$, a function can be generated which relates a particular value of $x$ to a particular random number $R$, where $0 \leq R \leq 1$ [2]:

$$
R=\int_{0}^{x} f(x) d x / \int_{0}^{\infty} f(x) d x
$$

In the case of the scattering angles, the ratio of the differential cross section to the total cross section can be integrated over all scattering angles to generate an appropriate function for selection of the scattering angles by random numbers:

$$
R=\int_{\Omega} \frac{\sigma^{-}(\phi)}{\sigma_{E}} d \Omega
$$

which leads to:

$$
\cos \phi=1-\left[2_{\alpha} R /(1+\alpha-R)\right]
$$

where $R$ is a random number $0 \leq R \leq 1$. The azimuthal scattering angle, $\psi$, has an equal - orobability at all values, and it can therefore be found from a simple equation:

$$
\psi=2 \pi R
$$

Thus, with the electron at a point $P_{N}$, having come from a point $P_{N-1}$, a set of scattering jarameters $(S, \phi$, and $\psi$ ) can be calculated. These values are employed with the appropriate geometrical expressions to obtain the new point, $\mathrm{P}_{\mathrm{N}+1}[2,3]$.

Energy loss during travel through the specimen is usually approximated by use of the 3ethe formula for continuous energy loss:

$$
\frac{d E}{d S}=-\frac{2 \pi e^{4} N A}{E} \quad \frac{Z}{A} \log \left(\frac{1.166 E}{J}\right)
$$


where $e$ is the electronic charge and $J$ is the mean ionization potential [5]. The energy loss $\Delta E$ along the path segment $\Delta S$, is:

$$
\Delta \mathrm{E}=\Delta \mathrm{S} \cdot \mathrm{dE} / \mathrm{dS}
$$

The single scattering form of the Monte Carlo simulation gives the most detailed information on the electron trajectory, since the step length of the calculation is almost identical to the mean free path. In addition, some workers have included a treatment of inelastic scattering as a discrete process of energy loss rather than using the continuous energy loss approximation [6]. While the single scattering approach is the most rigorous, it is also the most expensive to calculate, requiring up to several thousand repetitions of the basic element per trajectory.

\section{Multiple Scattering}

To reduce the time necessary for a calculation, several workers have used a multiple scattering approach. In this method, the effects of ten to twenty single scattering events are approximated by a "multiple scattering" step which, on average, produces the same net displacement and angular deviation as the sum of single scattering displacements within the step. The step length in a multiple scattering calculation is approximately ten times longer than that used in a single scattering calculation, which reduces the time for the calculation by about an order of magnitude. While the multiple scattering model has been found to give good results for flat bulk targets [3], it does not work well for thin films or small particles where the dimension of the target approaches the step length of the calculation. For such situations, the coarse description of the trajectory by the multiple scattering simulation is inadequate, and a single scattering procedure becomes necessary.

3. Hybrid Model

To overcome the limitations of the multiple scattering model while retaining most of the calculational speed, a hybrid model was developed which combines elements of both the single scattering and the multiple scattering models [7]. The initial portion of the trajectory from the incident energy, $\mathrm{E}_{0}$, to an energy of $0.95 \mathrm{E}_{0}$ is calculated with the single scattering simulation, after which the balance of the trajectory is calculated with the multiple scattering model. The trajectory is thus simulated with small steps and a more realistic model near the surface, and with large steps deeper in the target where high accuracy is less important. This hybrid Monte Carlo model has been found to give more accurate results than the pure multiple scattering model while retaining most of its calculational speed.

\section{Monte Carlo Calculations of Particles}

In particles with dimensions which are smaller than the interaction volume in a bulk target of the same composition, loss of electrons can occur from al1. surfaces. Transmission through the particle can take place as well as scattering through the sides and through the surface through which the beam entered. These escaping electrons are collectively called "backscattered" electrons in analogy to the bulk specimen situation. A description of this electron escape in terms of analytical equations would be difficult. The Monte Carlo electron trajectory simulation, however, is especially well suited to the calculation of electron tron beam - specimen interactions in particles. The calculated coordinates of each scattering point along the electron trajectory can be compared with the function describing the surface of the particle to determine when the electron escapes. Examples of electron trajectories within particles are shown in figure 3 where increasing the beam energy incident on spheres of aluminum leads to penetration of electrons through the entire particle.

\section{Applications to X-Ray Microanalysis of Particles}

As illustrations of the utility of Monte Carlo electron trajectory calculations for particulate analysis, three applications will be described in detail: 

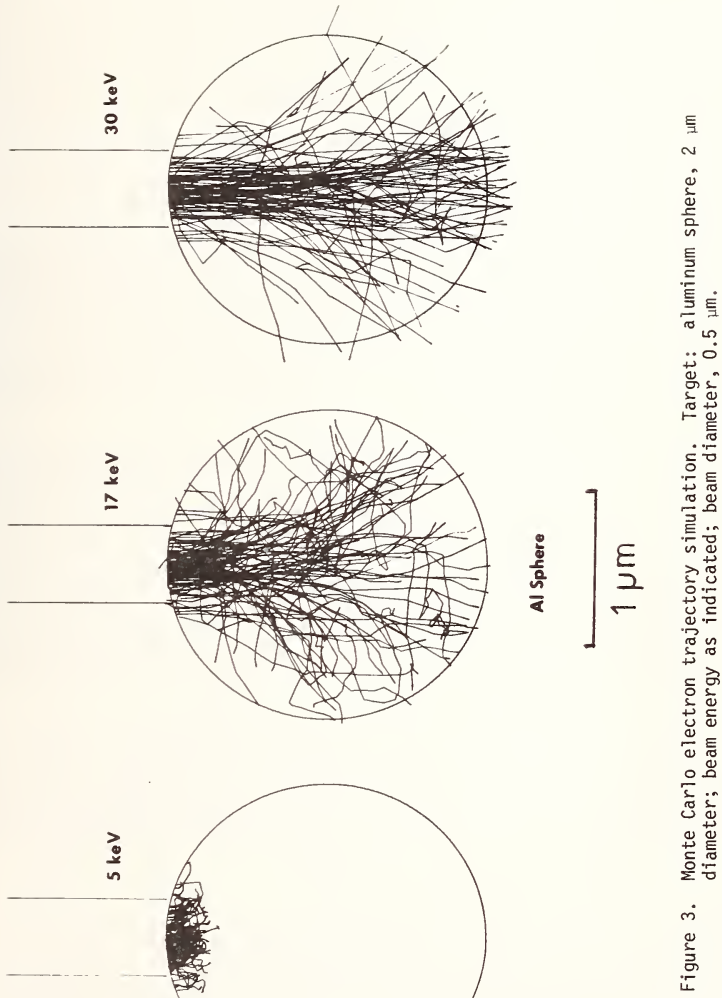
The $x$-ray emergence angle is defined as the angle above the surface at which the $x$-rays emerge in the direction of the detector (i.e., in the conventional arrangement of a flat specimen the complement of the angle between the beam axis and the detector axis), figure 4(a). The value of the emergence angle determines the length of path which the $x$-rays must traverse to escape the specimen and along which absorption takes place. In an electron probe microanalyzer, the emergence angle for both the crystal spectrometers and energy dispersive spectrometer is typically in the range 30-60 for a flat specimen set normal to the beam. [Such an instrument is thus capable of analyzing a specimen whose surface is positioned normal to the beam.] In a scanning electron microscope, the energy dispersive spectrometer is frequently attached with the detector axis at a right angle to the beam, which produces for a specimen of surface normal to the beam an x-ray emergence angle of $0^{\circ}$. Such a detector configuration cannot be applied to the analysis of a flat bulk specimen set normal to the beam. X-ray microanalysis of flat specimens is accomplished in such instruments by tilting the specimen toward the detector to create a sufficiently large emergence angle (figure 4(b)). While this is a satisfactory situation for a flat bulk sample, tilting does not necessarily produce an adequate emergence angle for a particle (figure $4(c))$. For a spherical particle with the beam directed towards the particle center, the emergence angle remains effectively $0^{\circ}$, even when the substrate carrying the particle is highly tilted. The degree to which this situation influences the measured $x$-ray intensity depends on the size of the interaction volume relative to the particle size. For very small particles, the extra $x$-ray path length caused by the $0^{\circ}$ emergence angle is not significant (figure $5(\mathrm{a})$ ). When the interaction volume becomes smaller than the particle size, figure $5(b)$, the added $x$-ray absorption path length can profoundly influence the measured x-ray intensity, particularly for radiation of long wavelength.

Monte Carlo simulations have been used to calculate the $x$-ray intensity which is emitted as a function of particle size for the situations illustrated in figures $4(\mathrm{c}$ ) and (d) with the beam incident at the center of the particle [8]. The intensities have been normalized with respect to the intensity calculated for a bulk substrate under the same conditions. The calculated response for a low energy x-ray line, silicon $\mathrm{K} \alpha(1.74 \mathrm{keV})$, in an iron matrix is shown in figure 6 for a cylindrical particle. For a $0^{\circ}$ emergence angle, a range of particle size is observed for which the normalized intensity is greater than unity; i.e., the particle emits more $x$-rays than the bulk sample. For larger particle sizes, the intensity rapidly drops off as the effect of the added absorption path length becomes significant. This behavior leads to an intensity function in which particles of two widely different diameters emit the same $x$-ray intensity. To the contrary, for a high emergence angle the $x-$ ray intensity does not fall off at large particle diameter, but it becomes identical with that observed from a bulk material.

The importance of the proper choice for the $x$-ray detector orientation in particle analysis is demonstrated by figure 6 . The dependence of the $x$-ray intensity on the sample geometry should be monotonic with respect to particle size since the intensity is also a complicated function of specimen composition, the determination of which is the analyst's goal. The sharp drop-off in intensity with increasing particle diameter observed for the $0^{\circ}$ angle case would make quantitative analysis unnecessarily difficult and therefore this configuration must be avoided.

\section{B. Particles and Films on Substrates}

To produce a specimen which is suitable for the analysis of individual particles, the particles are often dispersed on a massive substrate of a low atomic number material such as beryllium or carbon. Similarly, in order to analyze a material in the form of a thin film or to create thin film standards, the material must be supported. A massive, low atomic number material can be used for this purpose. Monte Carlo calculations can be useful in understanding the signals observed from particles or films on substrates. The trajectories of electrons and the signals which they generate can be calculated for the particle and film as well as the substrate. Thus, the case in which an electron penetrates through a particle or $f i 1 \mathrm{~m}$, enters the substrate, and is subsequently scattered out of the substrate to re-enter the particle or film can be be adequately treated. 

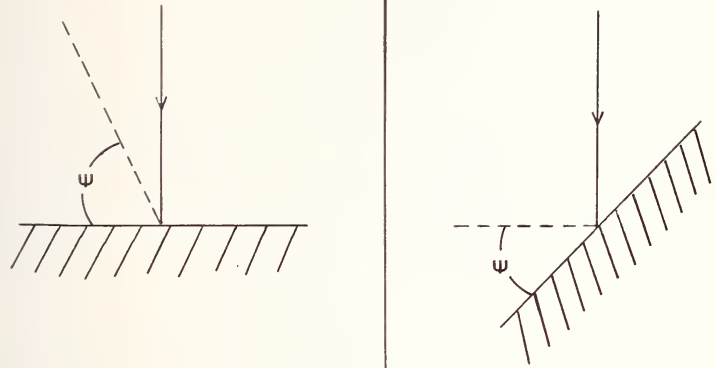

(a)

(b)
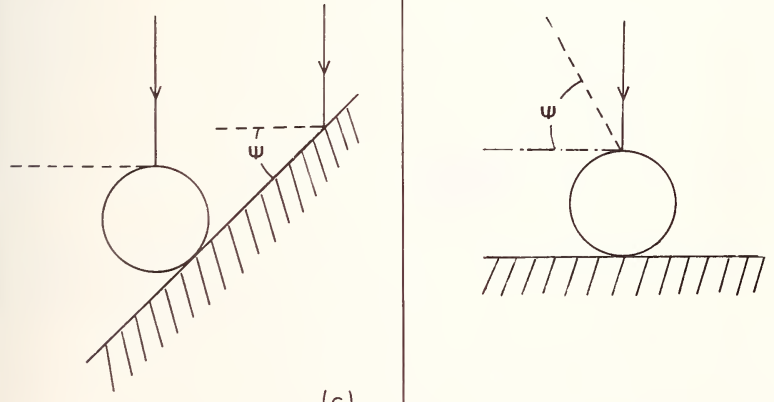

(c)

(d)

Figure 4. X-ray emergence angle in:

a. Solid specimen in electron probe microanalyzer.

b. Solid specimen in scanning electron microscope (detector at a right angle to beam axis).

c. Particle observed with SEM x-ray geometry.

d. Particle observed with electron probe x-ray geometry. 

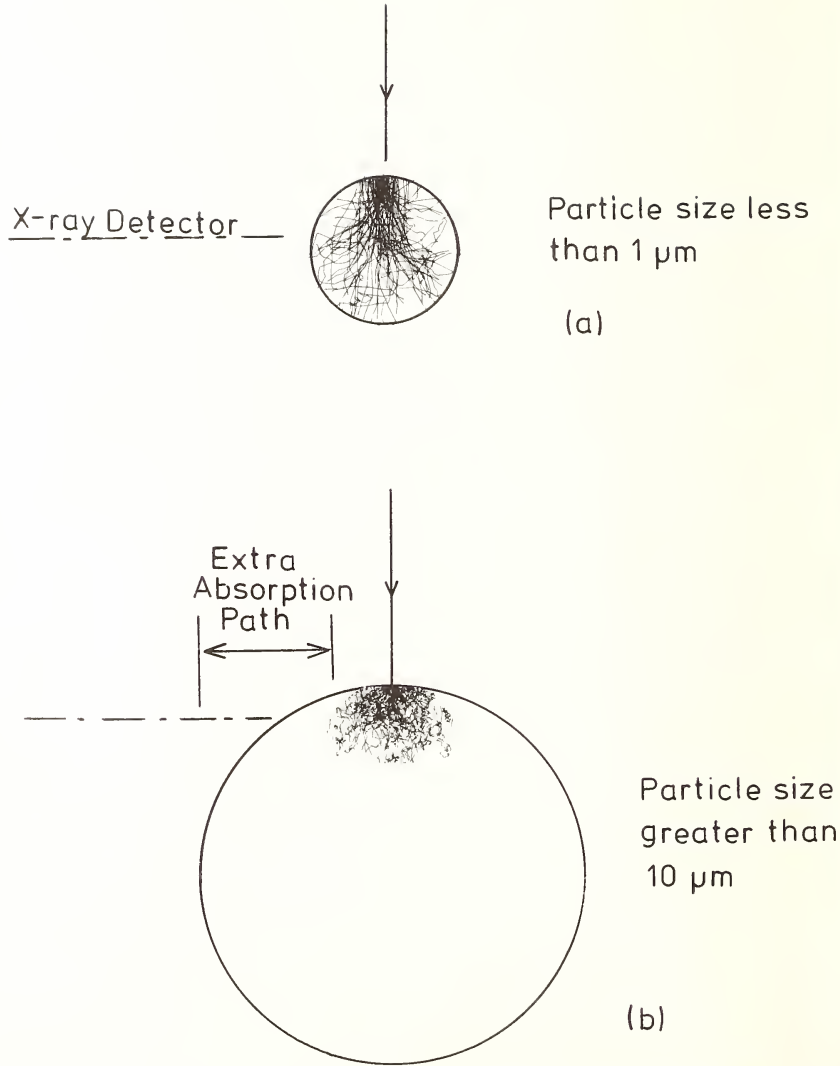

Figure 5. Illustration of the extra x-ray absorption path length in a large particle (b) viewed with an $x$-ray detector at a right angle to the beam axis compared to a small particle (a) which is totally excited by the beam. 


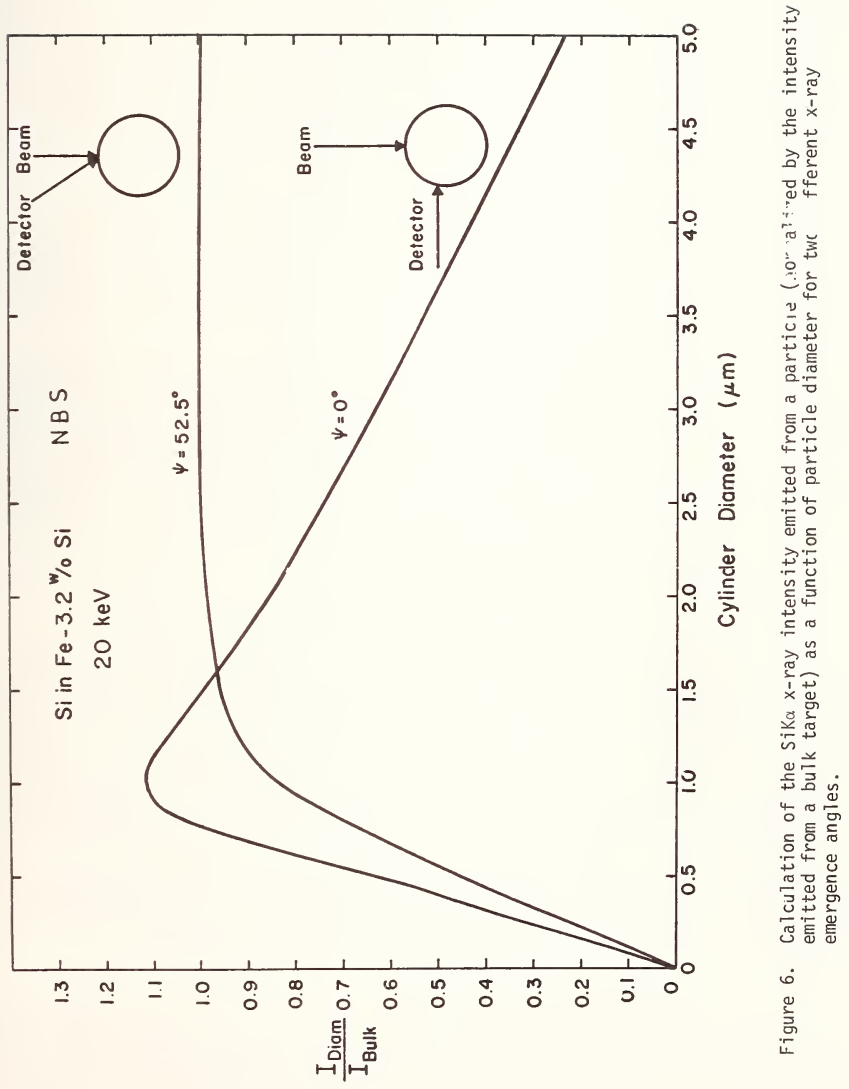


As an example of such an application, the characteristic x-ray signals emitted from thin sodium chloride layers on a beryllium substrate have been calculated by Monte Carlo techniques. Experimental results on similar specimens are reported by Roinel et al. [17] elsewhere in these proceedings. The target was assumed to consist of a matrix of sodium chloride doped with low levels of magnesium, potassium, and phosphorus, and the specimen surface was normal to the beam. Films of various thicknesses were considered and the $x$-ray intensities per unit thickness were normalized with respect to those calculated for the minimum thickness considered $\left(0.025 \mathrm{mg} / \mathrm{cm}^{2}\right)$. The observed working curves, figure 7 , reveal an initial increase in the relative efficiency of $x$-ray production as the thickness increases, followed by a decrease at greater thicknesses. The calculated results are similar for films on a beryllium substrate and without a substrate, except that the increase in efficiency is more exaggerated for the films without a substrate. The experimental results in the paper of Roinel et al. [17] do not show the initial increase in efficiency.

On a theoretical basis, the increase in efficiency with layer thickness can be understood to occur as a result of elastic scattering. Thus, the electron path through a film of twice the thickness increases by more than a factor of two since elastic scattering causes the electrons to deviate from their initial path normal to the foil. The additional path length leads to greater $x$-ray production and hence, more efficient production per unit thickness. The eventual decrease which is observed occurs because of absorption effects and loss of electron energy. An increase in efficiency is observed as a function of $x$-ray energy; this can be explained in terms of overvoltage effects.

The deviation of the Monte Carlo calculations from the results of the experiments of Roinel et a1. [17] indicates that the film model is in disagreement with the experimental situation. Scanning electron micrographs of the microdroplet targets made by Roinel et al. reveal that the specimen is formed of small $(\sim 1 \mu \mathrm{m}$ diameter) crystallites rather than being a uniform thin film. If the main effect of mass increase of the specimen is an increase of the number of particles of approximately constant diameter, a wide range of linearity of the calibration curve, with gradual decrease of relative efficiency, can be predicted. Such a behavior was, in fact, observed experimentally by the above authors [17].

Monte Carlo calculations are thus useful in understanding the behavior of $x$-ray signals emitted from complex targets.

\section{Calculations of Ratios of Characteristic-to-Continuous X-ray Intensities}

It was reported simultaneously by this group [9] and by Statham and Pawley [10] that experimental measurements of the ratio of characteristic $x$-rays to continuous $x$-rays of similar energy (peak-to-background ratio) are nearly identical in particles and bulk material of the same composition over a wide range of particle size. This experimental observation has been used to develop a scheme for particle analysis based on the ZAF (atomic numberabsorption-fluorescence) matrix correction method. The particle analysis scheme has been reported previously in outline form $[9,11]$ and is described in detail elsewhere in this volume [12]. In order to aid the development and refinement of this method, the Monte Carlo technique has been applied to the problem of calculating the peak-to-background ratios for $x$-rays emitted from particles.

To carry out these calculations, an appropriate cross section is needed for the production of both the continuous $x$-rays and the characteristic x-rays. Selection and testing of cross sections for Monte Carlo simulations to calculate absolute $x$-ray yields have not been previously reported, and therefore these topics will be considered briefly.

\section{(1) Continuum generation cross sections}

Kirkpatrick and Wiedmann [13] have derived algebraic expressions for the production of $x$-ray continuum intensity as a function of continuum energy and beam energy. Statham [14] has modified slightly the expressions of Kirkpatrick and Wiedmann over certain ranges and incorporated the resulting cross section in a Monte Carlo simulation. In the present work, we have used the original Kirkpatrick and Wiedmann [13] expressions to calculate the intensity of the continuous x-radiation emitted from bulk and particle targets. Since the generation of continuous radiation is related to the direction of electron travel, the 


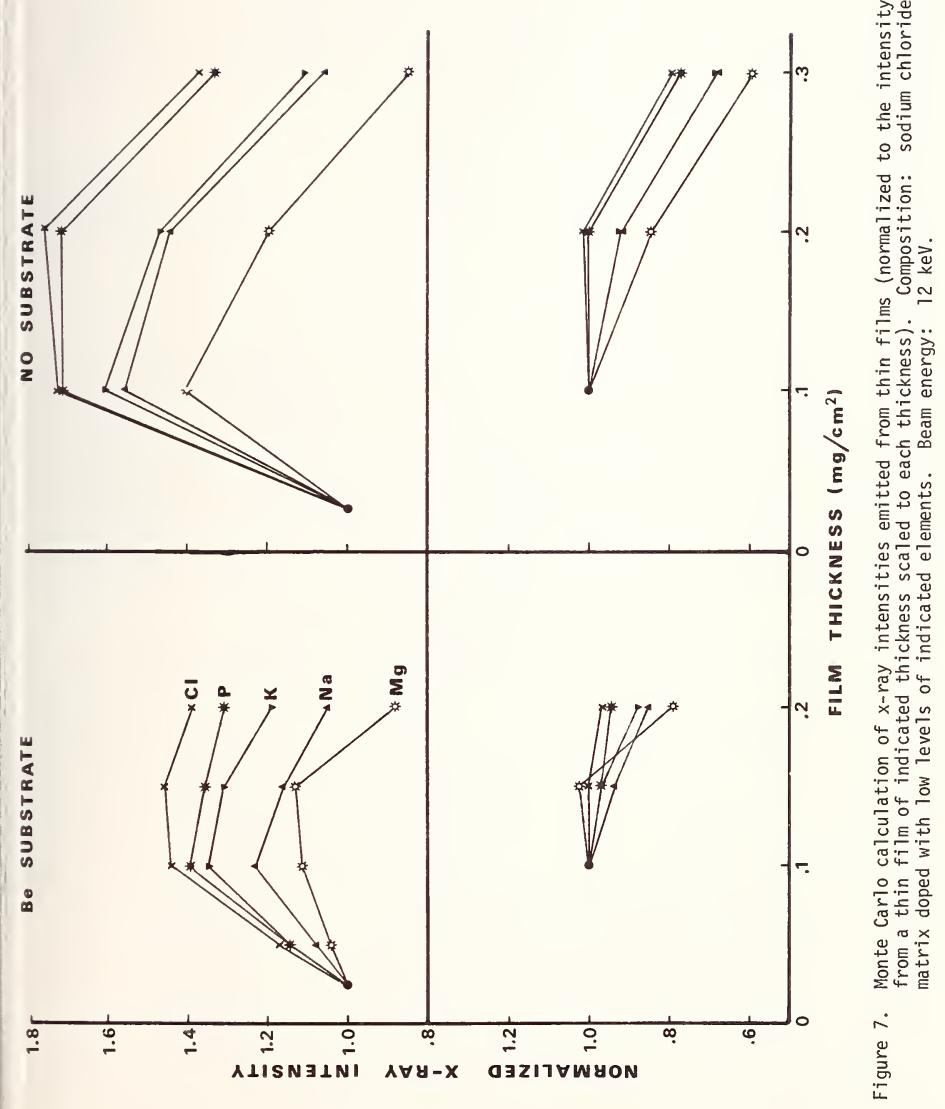


anisotropic form of the continuum cross section has been used. For comparison purposes, the cross section obtained when the emission is assumed to be isotropic has also been calculated.

The correspondence of the calculated continuous spectrum derived from the anisotropic cross section with that measured for a bulk target is found to be quite good. Our calculated and experimentally determined continuum spectra for titanium are shown in figure 8. The calculated spectrum has been corrected for absorption in the sample and for absorption in the beryllium window and silicon dead layer of the energy dispersive spectrometer. Similar correspondence between calculation and experiment has been found for bulk targets of copper and gold. These studies suggest that the technique for calculation of the continuum spectrum is satisfactory for the present studies.

\section{Characteristic X-ray Emission Cross Section}

In contrast with the situation for the continuous $x$-rays, the calculation of the intensity of characteristic $x$-rays generated in the sample is somewhat less satisfactory. A number of expressions for the cross section are available in the literature [15]. These have the form

$$
Q=\frac{c_{1} Z_{n 1} C_{2}}{E_{c}^{2}} \frac{\log _{e}\left(c_{3} U\right)}{C_{4}\left(U+c_{5}\right)} \quad C_{6}
$$

where $C_{1}=6.51 \times 10^{-20} \mathrm{~cm}^{2} \mathrm{keV}^{2}$ (where $C_{1}$ is $\pi \mathrm{e}^{4}$, with e the electronic charge), $Z_{n 1}$ is the number of electrons in the shell with critical ionization energy $E_{C}(k e V), U$ is the overvoltage ( $U=E / E_{C}$, where $E$ is the energy of the electron), and $C_{2}, C_{3}, C_{4}, C_{5}$, and $C_{6}$ are constants with different values depending on the choice of cross section model. By setting some of these constants equal to unity, all of the models can be realized. Lifshin et al. [16] recently reported experimental values of the total characteristic intensity generated in bulk, pure element targets as a function of overvoltage. We have calculated the total characteristic intensity predicted by the Monte Carlo simulation for similar sample and beam conditions with several different choices of expression for the ionization cross section. The values are compared in Table 1. In general, for the cross sections tested, no completely satisfactory fit to the experimental data is obtained.

Table 1. Characteristic $x$-ray generation (photons/electron)

$$
E=20 \mathrm{keV} \text {. }
$$

Monte Carlo Calculations

$\begin{array}{lcc} & \text { Nickel } & \text { Titanium } \\ \text { Cross Section } & \left(\times 10^{-4}\right) & \left(\times 10^{-4}\right) \\ \text { Fabre } & 0.80 & 2.38 \\ \text { Bethe (Mott and Massey) } & 1.54 & 2.84 \\ \text { Green and Cosslett } & 0.981 & \\ \text { Experimental Measurements } & & 3.36 \\ \text { Lifshin } & 1.53 & 2.4 \\ \text { Green } & - & \end{array}$




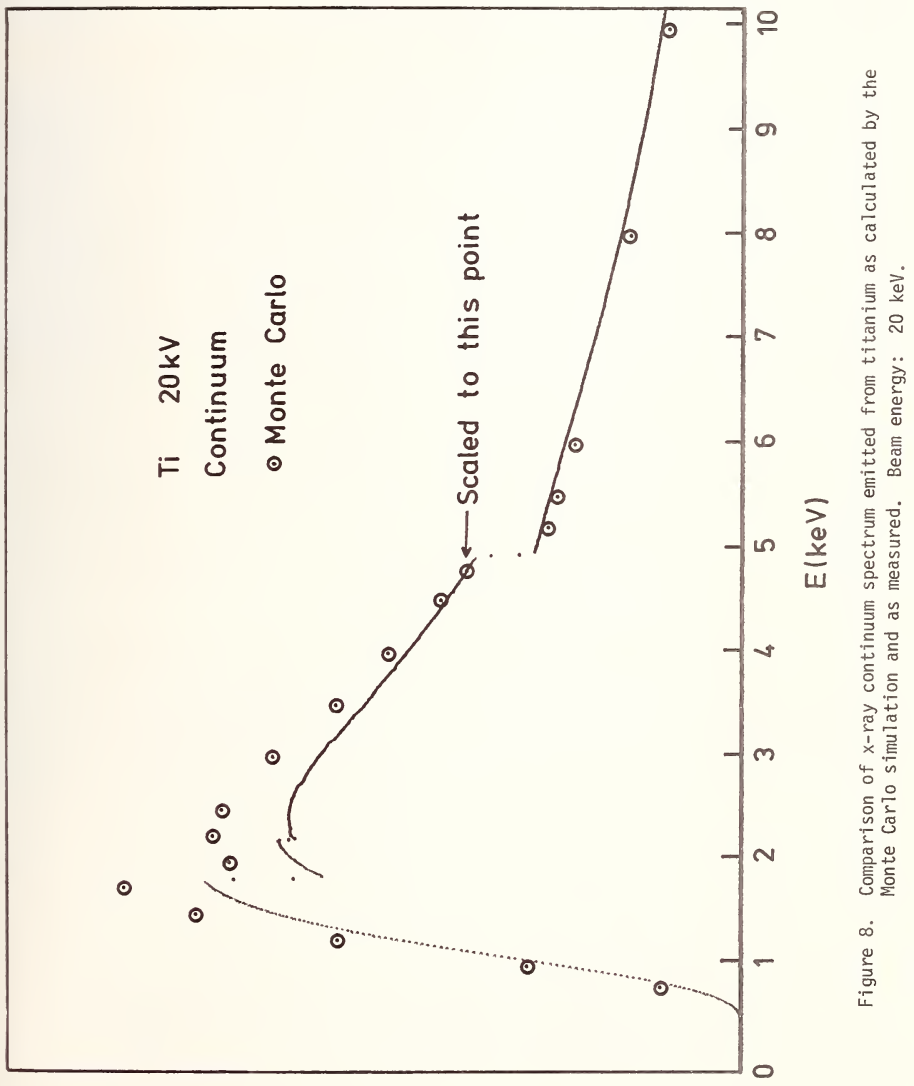


For the particle calculations, ratios of characteristic intensities are employed, and therefore the effects of the choice of constants which regulate the absolute intensity of the calculated characteristic $x$-rays tend to cancel within the accuracy required for the present case. Similarly, the choice of the stopping power has 1ittle effect in the case of calculating ratios. Several different models for cross sections for characteristic $x$-ray production have been examined in the following work, and the results for the ratios have been found to be essentially independent of the exact model.

\section{Comparison of Peak-to-Background Ratios in Particle and Bulk Material}

To test the experimental observation that the peak-to-background is similar in particles and bulk material, a hypothetical target of the same composition as NBS Glass K-309 was considered. This composition has the advantage of providing characteristic $x$-ray energies spanning the range $1.5-6.4 \mathrm{keV}$ and resembles natural silicate particles. Peak-to-background $(P / B)$ calculations were made for the bulk material and for spherical particles of various diameters. Al1 Monte Carlo calculations were made with a beam with a constant current density of the same size as the particle. This condition corresponds closely to the situation achieved experimentally with raster scanning of particles [9]. The results for a particle of $6 \mu \mathrm{m}$ diameter, reported as a ratio of $(\mathrm{P} / \mathrm{B})_{\text {particle }}$ to $(\mathrm{P} / \mathrm{B})_{\text {bulk, }}$, are given in Table 2. For this $6 \mu \mathrm{m}$ diameter particle, the $(P / B)$ values at $20 \mathrm{keV}$ are found to be quite similar to that of the bulk, with a value slightly higher by 2-6 percent for the particle. The ratios of peak intensities emitted by the particle and bulk, also listed in Table 2, show much larger deviations, with low energy lines actually being emitted with greater intensity from the particle than from the bulk.

Table 2. Comparison of $6 \mu \mathrm{m}$ particle to bulk (K309)

$$
\frac{(P / B)_{\text {particle }}}{(P / B)_{\text {bulk }}}
$$

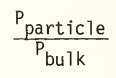

Element

$\begin{array}{lll}\text { A1 } & 1.03 & 1.12 \\ \mathrm{Si} & 1.00 & 1.06 \\ \mathrm{Ca} & 1.02 & 0.86 \\ \mathrm{Ba} & 1.06 & 0.86 \\ \mathrm{Fe} & 1.05 & 0.87 \\ \mathrm{E}_{0}=20 \mathrm{keV} & & \\ \text { emergence angle }=40^{\circ} & & \end{array}$

Note: Calculated with Monte Carlo simulation with beam at the center of the particle. 


\section{(a) Effect of Particle Size on (P/B) Ratios}

When the ratio of $(P / B)$ ratios is calculated as a function of particle size (Table 3 ), 1 systematic deviation is observed. The $(P / B)$ ratio of the particle increases relative to hat of the bulk material as the particle size decreases. This increase in the ( $P / B$ ) ratio if the particle occurs over the entire energy range which was studied.

\begin{tabular}{|c|c|c|c|c|c|c|}
\hline \multirow[b]{2}{*}{ Element } & \multirow{2}{*}{$\begin{array}{c}\text { Line } \\
\text { Energy } \\
\mathrm{keV} \\
\end{array}$} & \multicolumn{5}{|c|}{ Diameter $(\mu \mathrm{m})$} \\
\hline & & $\underline{6}$ & $\underline{3}$ & $\underline{2}$ & 1 & $\underline{0.5}$ \\
\hline Al & 1.48 & 1.03 & 1.10 & 1.21 & 1.39 & 1.51 \\
\hline Si & 1.74 & 1.00 & 1.05 & 1.14 & 1.29 & 1.38 \\
\hline $\mathrm{Ca}$ & 3.69 & 1.02 & 1.10 & 1.22 & 1.43 & 1.54 \\
\hline $\mathrm{Ba}$ & 4.46 & 1.06 & 1.14 & 1.30 & 1.55 & 1.67 \\
\hline $\mathrm{Fe}$ & 6.40 & 1.05 & 1.11 & 1.31 & 1.54 & 1.67 \\
\hline
\end{tabular}

Electron range at $20 \mathrm{kV}$ is approximately $3.1 \mu \mathrm{m}$.

(b) Effect of Shape

Calculations of the $(P / B)$ ratio have been made for both spheres and cylinders of nfinite length, of two different diameters, as compared to bulk material (Table 4). The $P / B$ ) values for the cylinders do not show as large a deviation from bulk values as do the sheres. This behavior occurs because the cylinder axis is effectively infinite as in a ulk specimen. 
Table 4. Comparison of spheres (SPH) and cylinders (CYL) of glass $\mathrm{K} 309$ at $20 \mathrm{kV}$ (emergence angle $=40^{\circ}$ )

$\frac{\text { Peak } / \mathrm{Bg}_{\text {Particle }}}{\text { Peak/Bg Bulk }}$

\begin{tabular}{|c|c|c|c|c|c|}
\hline \multirow[b]{3}{*}{ Element } & \multirow{3}{*}{$\begin{array}{c}\text { Line } \\
\text { Energy } \\
\text { keV } \\
\end{array}$} & \multicolumn{4}{|c|}{ Diameter $(\mu \mathrm{m})$} \\
\hline & & \multicolumn{2}{|c|}{6} & \multicolumn{2}{|c|}{1} \\
\hline & & $\underline{\mathrm{SPH}}$ & $\underline{C Y L}$ & $\underline{\mathrm{SPH}}$ & $\underline{\mathrm{CYL}}$ \\
\hline Al & 1.48 & 1.03 & 1.01 & 1.39 & 1.21 \\
\hline Si & 1.74 & 1.00 & 0.98 & 1.29 & 1.14 \\
\hline $\mathrm{Ca}$ & 3.69 & 1.02 & 1.00 & 1.43 & 1.24 \\
\hline $\mathrm{Ba}$ & 4.46 & 1.06 & 1.02 & 1.55 & 1.31 \\
\hline $\mathrm{Fe}$ & 6.40 & 1.05 & 1.01 & 1.54 & 1.33 \\
\hline
\end{tabular}

\section{(c) Effect of Beam Energy}

The systematic deviation in the $(\mathrm{P} / \mathrm{B})$ ratio of the particle is a function of the energy of the incident beam, and hence the range of the electron in the target. Calculations for three different particle sizes at incident beam energies of 10 and $20 \mathrm{keV}$ are given in Table 5. A reduction in the beam energy by a factor of two reduces the deviation from bulk behavior by at least a factor of two.

Table 5. Effect of beam energy on peak-to-background ratios ( $\mathrm{K} 309 \mathrm{glass}$, emergence angle $=40^{\circ}$ )

$$
\frac{\text { Peak/Bg }}{\text { Peak/Bghere }}
$$

\begin{tabular}{|c|c|c|c|c|c|c|c|}
\hline \multirow[b]{3}{*}{ Element } & \multirow{3}{*}{$\begin{array}{c}\text { Line } \\
\text { Energy } \\
\text { keV }\end{array}$} & \multicolumn{6}{|c|}{ Diameter $(\mu \mathrm{m})$} \\
\hline & & \multicolumn{2}{|c|}{3} & \multicolumn{2}{|c|}{1} & \multicolumn{2}{|c|}{0.5} \\
\hline & & $20 \mathrm{kV}$ & $10 \mathrm{kV}$ & $20 \mathrm{kV}$ & $10 \mathrm{kV}$ & $20 \mathrm{kV}$ & $10 \mathrm{kV}$ \\
\hline Al & 1.48 & 1.10 & 1.01 & 1.39 & 1.06 & 1.51 & 1.21 \\
\hline Si & 1.74 & 1.05 & 1.01 & 1.29 & 1.05 & 1.38 & 1.18 \\
\hline $\mathrm{Ca}$ & 3.69 & 1.10 & 1.01 & 1.43 & 1.08 & 1.54 & 1.27 \\
\hline $\mathrm{Ba}$ & 4.46 & 1.14 & 1.01 & 1.55 & 1.11 & 1.67 & 1.33 \\
\hline $\mathrm{Fe}$ & 6.40 & 1.11 & 1.01 & 1.54 & 1.08 & 1.67 & 1.31 \\
\hline
\end{tabular}




\section{Origin of Deviation}

The deviation in the $(P / B)$ ratio of the particle from $(P / B)$ ratio of the bulk apparitly occurs for the following two reasons:

\section{Anisotropy of Generation of Continuous X-rays}

The generation of $x$-ray continuum along a segment of electron trajectory is anisotropic cause it is related to the direction of flight of the electron and is peaked in a forward rection. In a bulk target, the effects of elastic scattering lead to a randomization of e directions of flight of the beam electrons, so that although the generation of continuum anisotropic along an individual electron trajectory, the generation averaged over the tire interaction volume appears nearly isotropic to an external detector. In a small rticle or thin film, the effects of elastic scattering are reduced when the dimensions come significantly less than the bulk interaction volume. The trajectory segments are re parallel, so that the anisotropic effects are not cancelled by randomization. Moreer, this anisotropy also explains the behavior of $(P / B){ }_{\text {particle }} /(P / B)_{b u l k}$ as a function beam energy. At high beam energy, the cross section for elastic scattering is reduced, e mean free path and total range increase, and the trajectory segments in a small rticle become more parallel (figure 3 ). The anisotropic generation effects are thus less bject to randomization by elastic scattering at high energy as compared to low energy.

This hypothesis can be tested by comparing the calculated behavior of (P/B) particle' (B) bulk as a function of particle size and considering both anisotropic and isotropic ntinuum generation. In figure 9, this comparison has been calculated for three different ements ( $\mathrm{Ca}, \mathrm{Fe}$, and $\mathrm{A} 1$ ) in $\mathrm{K}-309 \mathrm{glass}$ spheres referenced to bulk material. In all cases, can be seen that about half the deviation of $(P / B)_{\text {particle }}$ from $(P / B)_{b u l k}$ is related to e anisotropy of the continuum generation.

\section{Difference in Cross Sections for Characteristic and Continuum Rays}

The remaining portion of the deviation from bulk $(P / B)$ values occurs as a result of the mplicated relation between the cross sections for characteristic and continuum cross ctions and the energy spectrum of electrons emitted from the particle. The cross sections $r$ characteristic and continuum $x$-radiation as a function of electron energy are shown in gure 10. As the electron energy is reduced and approaches the $x$-ray energy of interest, e cross section for characteristic $x$-ray production decreases while the cross section for ntinuum $x$-ray production increases. Beam electrons which have lost sufficient energy to ach the range of a few $\mathrm{keV}$ above the $x$-ray energy of interest make contributions prinpally to the continuum and not to the characteristic x-radiation. These lower energy ectrons therefore tend to reduce the peak-to-background ratio. In a particle, the effect electron penetration is to allow the escape of high energy electrons before ihey have the portunity to lose large amounts of energy. The energy spectrum of electrons backscattered om a particle as calculated with our Monte Carlo simulation is seen to be more sharply aked at a higher fraction of the initial energy than that from the bulk specimen (figure ). Thus, in a particle, most electrons do not reach the lower energies necessary to ficiently create continuous $x$-rays of the energy of interest. The (P/B) ratio for the rticle is therefore expected to be higher than that for the bulk since less continuum is eated relative to the characteristic emission. This effect increases as the particle size comes smaller.

The deviation of $(P / B)_{\text {particle }}$ from $(P / B)_{\text {bulk }}$ therefore originates from two effects, e anisotropy of continuum emission and the difference in the relative behavior of the oss sections for continuous and characteristic $x$-ray generation. Both of these effects e a function of the particle size as a fraction of the interaction volume in the bulk terial. The deviation of $(P / B)_{\text {particle }}$ from $(P / B)_{\text {bulk }}$ should thus have the form 


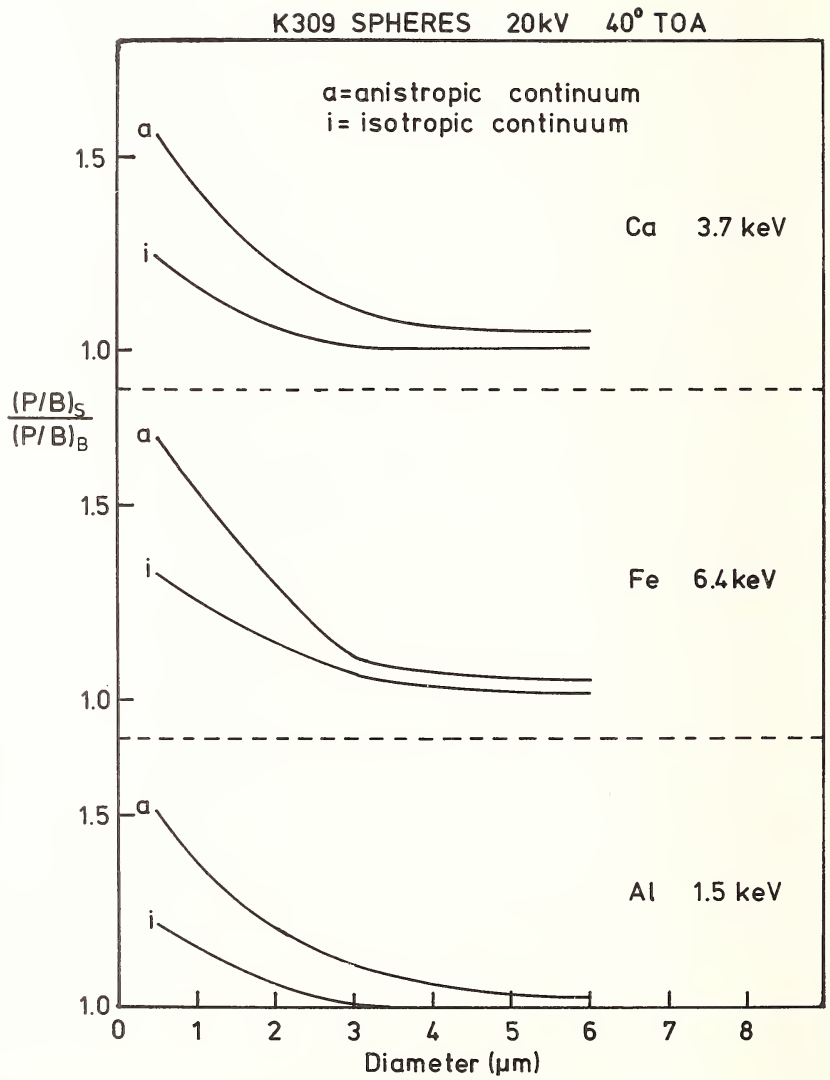

Figure 9. Monte Carlo calculations of the peak/background ratio emitted from a spherical particle (normalized by $(P / B)$ for a bulk target) as a function of particle diameter. Both the isotropic and the anisotropic continuum cases are considered. Target: K-309 glass; beam energy: $20 \mathrm{keV}$; x-ray emergence angle $40^{\circ}$. 


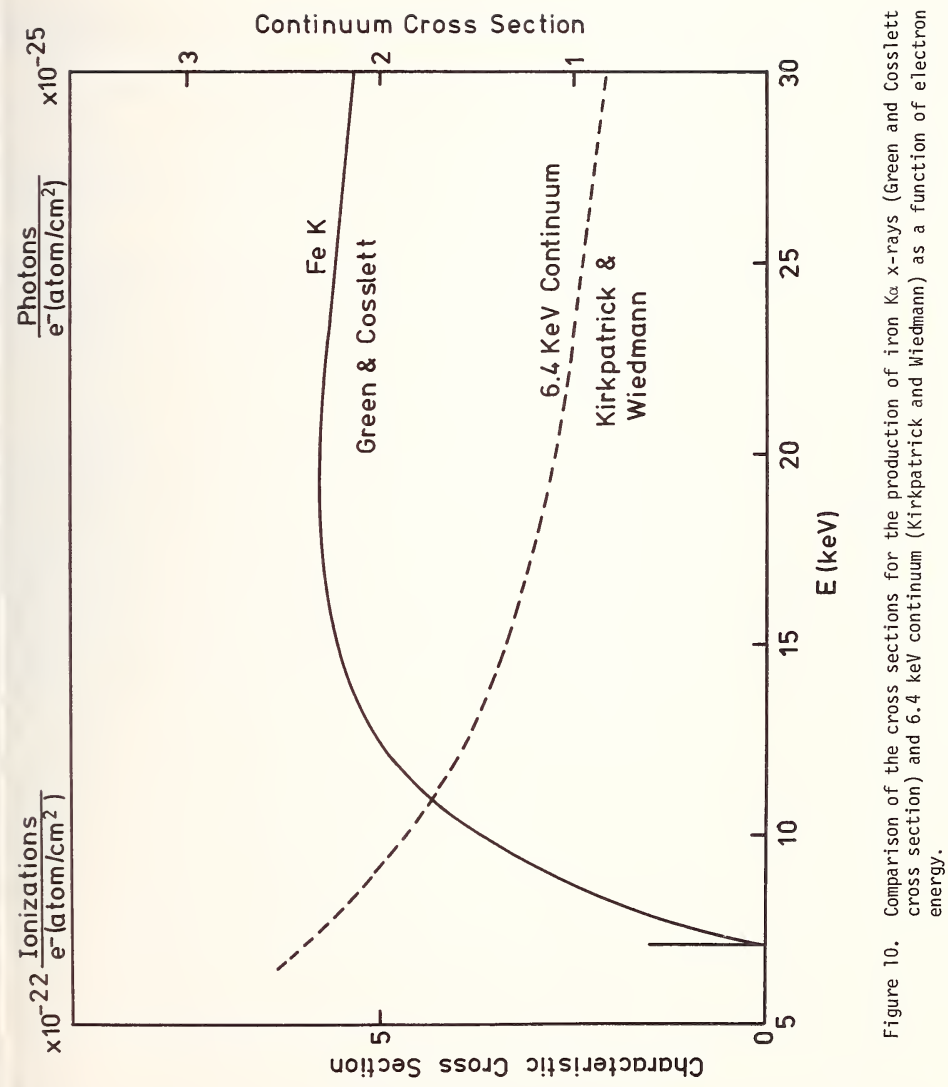




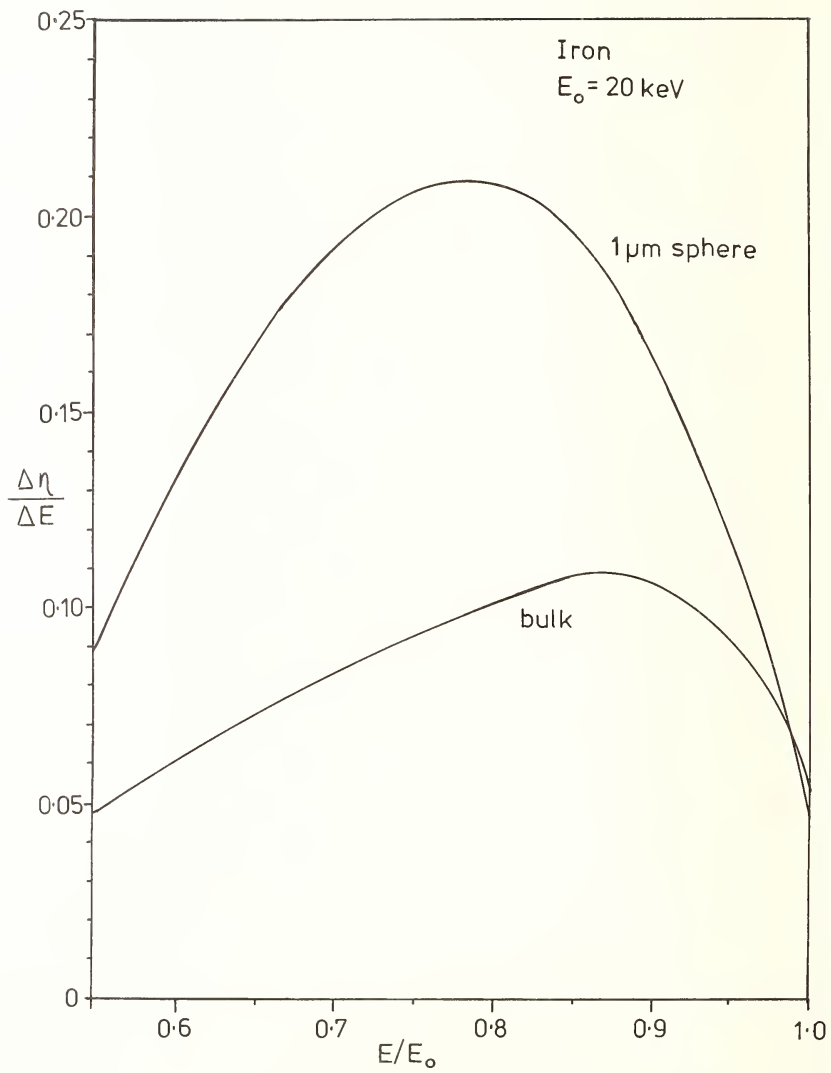

Figure 11. Energy spectra of beam electrons which escape a bulk iron target and an iron spherical particle ( $1 \mu \mathrm{m}$ diameter). Beam energy: $20 \mathrm{keV}$. Monte Carlo calculation. 


$$
\frac{(P / B)_{\text {bulk }}}{(P / B)_{\text {particle }}}=f\left(\frac{\text { diameter particle }}{\text { range }_{\text {bulk }}}\right) \text {, diameter }<\text { range. }
$$

a first approximation, the behavior is similar for all $x$-ray energies of interest, and erefore one factor can be used to adjust the $(P / B)$ particle value measured from the actual
ectrum for the deviation:

$$
\left.\left[(\mathrm{P} / \mathrm{B}){ }_{\text {particle }}\right] \underset{\left(\begin{array}{l}
\text { anisotropy } \\
\text { energy }
\end{array}\right.}{\text { and }}=\left[(\mathrm{P} / \mathrm{B})_{\text {particle }}\right] \text { (measured }\right) \times \mathrm{F}
$$

can be observed by examining the form of the curves in figure 9, the correction $F$ is osely approximated by the function:

$$
F=A e^{-B X}+C
$$

ere $X=D / R_{B}, D$ is the particle diameter, and $R_{B}$ is the Bethe range in the bulk material. $r$ the example of the $K-309$ glass in figure 9, the values of the constants are $A=0.75$, $=2$, and $C=1$. The generality of this equation as a modification to the particle alys is scheme is currently being tested.

\section{Summary}

The technique of Monte Carlo electron trajectory simulation forms a valuable aid to rticle analysis. The cost of computation makes the direct application of the Monte Carlo mulation to a particular analysis problem justifiable in only a few cases. The principal lue of Monte Carlo simulation is as a tool for the development of direct computational hemes for particle analysis. The Monte Carlo simulation can provide calculated data where | perimental data are difficult to obtain. In addition, the Monte Carlo simulation can equently be employed to separate effects, such as the influence of the anisotropy of the ntinuum $x$-ray emission in the present studies, which would be virtually impossible to parate experimentally from other factors which effect the $x$-ray generation. In applying e Monte Carlo simulation, it is important to remember that the calculated results are eatly influenced by the choice of scattering models, mean ionization potential, and other rameters. It is therefore necessary to test the calculational scheme as completely as ssible before confidence can be placed in the results of the simulation. 
[1] Heinrich, K. F. J., Newbury, D. E., and Yakowitz, H., eds., Use of Monte Carlo Calculations in Electron Probe Microanalysis and Scanning Electron Microscopy, NBS (U.S.), Spec. Publ. 406 (Washington, D.C. 1976).

[2] Berger, M. J., Methods in Computational Physics, Vol. 1, Alder, B., Fernback, S., and Rothenberg, M., eds., Academic Press, New York (1961).

[3] Myklebust, R. L., Newbury, D. E., and Yakowitz, H., Use of Monte Carlo Calculations in Electron Probe Microanalys is and Scanning Electron Microscopy, NBS (U.S.), Spec. Publ. 406, 105 (Washington, D. C. 1976).

[4] Reimer, L. and Krefting, E. R., Ibid., 45.

[5] Bishop, H. E., Ibid., 5.

[6] Shimizu, R., Kataoka, Y., Ikuta, T., Koshikawa, T., and Hashimoto, H., J. Phys. D: Appl. Phys. $\underline{9}, 101$ (1976).

[7] Newbury, D. E., Myklebust, R. L., and Heinrich, K. F. J., in Eighth International Conference on X-ray Optics and Microanalysis, Ogilivie, R. and Wittry, D., eds., (Boston, MA 1976) 27A.

[8] Myklebust, R. L., Newbury, D. E., and Heinrich, K. F. J., Proc. 11th Annual Conference, Microbeam Analysis Society (Miami, FL 1976) 23A.

[9] Small, J. A., Heinrich, K. F. J., Fiori, C. E., Myklebust, R. L., Newbury, D. E., and Dilmore, M. F., Scanning Electron Microscopy, Johari, 0., ed., Vol. 1, 445 (1978).

[10] Statham, P. J. and Pawley, J. B. , Ibid, 469.

[11] Smal1, J., Heinrich, K. F. J., Fiori, C., Newbury, D., and Myklebust, R., Proc. 13th Annual Conf. Microbeam Analysis Society (Ann Arbor, MI 1978) 61.

[12] Smal1, J., Heinrich, K. F. J., Myklebust, R. L., and Newbury, D., this volume.

[13] Kirkpatrick, P. and Wiedmann, L., Phys. Rev., 67, 321 (1945).

[14] Statham, P. J., X-ray Spectr., 5, 154 (1976).

[15] Powe11, C. J., Use of Monte Carlo Calculations in Electron Probe Microanalysis and Scanning Electron Microscopy, NBS (U.S.), Spec. Pub1. 406, 97 (Washington, D. C. 1976)

[16] Lifshin, E., Ciccarelli, M. F., and Bolon, R. B., 12th Annual Microbeam Analysis Society (Boston, MA 1977) 104A.

[17] Roine1, N., Champigny, M. , Meny, L., and Henoc, J., this volume. 
tional Bureau of Standards Special Publication 533. This Publication Originated from a ecial Session on Particle Analysis, 13th Annual Conference of the Microbeam Analysis ciety, Ann Arbor, MI, June 22, 1978. (Issued Apri1 1980)

\title{
QUANTITATIVE CHARACTERIZATION OF PARTICULATES BY SCANNING AND HIGH VOLTAGE ELECTRON MICROSCOPY
}

\author{
R. J. Lee and R. M. Fisher \\ U. S. Steel Corporation \\ Research Laboratory \\ Monroeville, PA 15146
}

\begin{abstract}
The development of a minicomputer-based automatic image analysis (AIA) method for scanning electron microscopy (SEM) now makes it possible to determine the size, shape, and composition of a large number of particles in a very short time. Thus, it is feasible to obtain statistically significant estimates of the bulk concentration and size distribution of specific classes of particles in powders, airborne dusts, and other particulate samples. This new technique provides an important link between bulk measurements by sieving and other methods and detailed studies of the morphology, internal structure, and composition of individual particles.
\end{abstract}

\section{Introduction}

"Analytical" microscopy is used to describe studies in which one or more of the ailable signals is recorded and interpreted in detail for a small number of particles or atures $[1,2]^{1}$. However, there is a large class of problems for which it is desirable to uantitatively" characterize a large number of particles. Powders, pigments, and airborne sts, as well as inclusions or second phases in steels, minerals, or coal, are just a few the many examples of particulate problems encountered in quality-control or processnitoring programs. Very often the differences between samples or batches are rather btle and can only be detected when statistical criteria are used to compare descriptive rameters such as size, shape, and area. In this case, the measurement and analysis of veral thousand particles may be required if the sample contains a mixture of several fferent types of particles having different size distributions.

The quantitative particulate analysis procedures to be described include three stinct phases: (1) automated measurement of particle parameters amenable to rapid alysis, such as size, shape, and composition, and assignment to type classes [3-6]; ) verification of the homogeneity and specific identity of the particle type comprising e class [7]; and (3) detailed analysis of the morphology, composition, crystal structure nd occurrence of defects) of particles belonging to a specific class of interest [8]. e ultimate goals of these methods are commensurate with those of image analysis and croscopy in general - to maximize the information obtained per particle while reducing the mplexity of the procedures, and to minimize the time required for analysis.

\section{Automatic Image Analysis by Scanning Electron Microscopy}

Figure 1 is a photograph showing a scanning electron microscope, Tracor Northern ftware-based $x$-ray analyzer, LeMont Scientific digital beam scan controller, display rminal, floppy disk data storage, and other computer peripherals comprising the SEM-AIA cility. A schematic diagram of the signal and information flow in the automatic image

igures in brackets indicate the literature references at the end of this paper. 


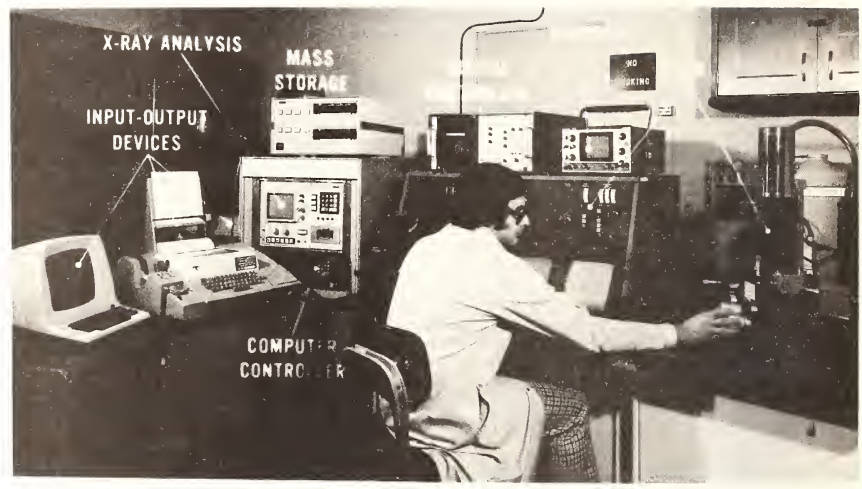

Figure 1. Scanning electron microscope equipped for automatic image analysis as installed in the USS Research Laboratory.

analysis mode is shown in figure 2. The minicomputer shown at the top of the figure directs the digital beam-control system for real-time picture-point by picture-point analysis (up to 4096 by 4096 matrix) as described below.

The energy-dispersive spectrometer (EDS) and software-based multichannel analyzer shown at the bottom of the illustration are used for $x$-ray microanalysis and classification of features. This system makes use of the same input/output and mass-storage devices as the minicomputer that controls the electron beam, which permits simultaneous collection of size, shape, and composition information from image features. A teletype (TTY) high-speed printer, magnetic-tape cassette, and dual floppy disk (not shown) are used as input/output and mass-storage devices. 


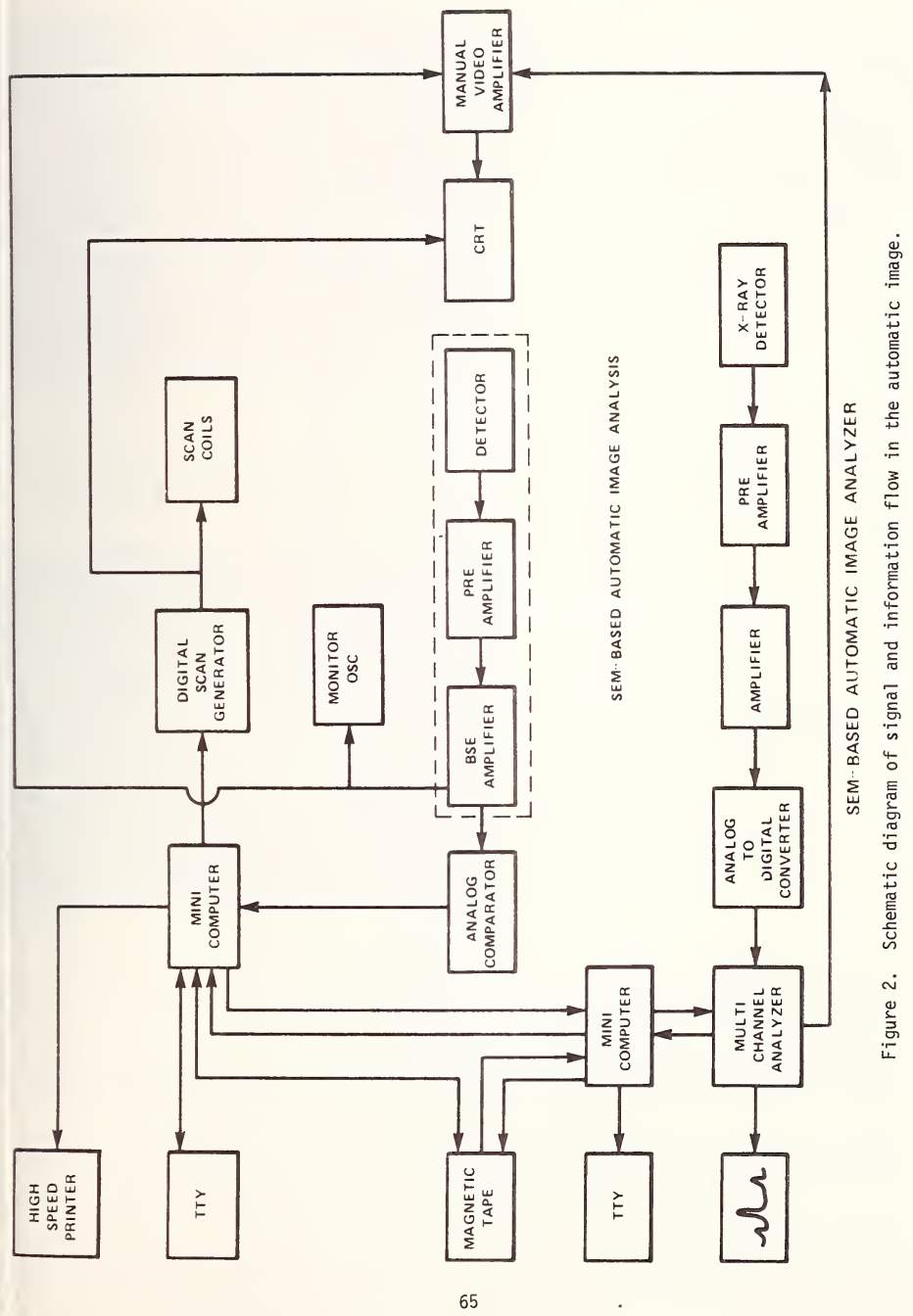


The time sequence of events comprising the automatic image analysis procedure is illustrated schematically in figure 3. The digital scan generator (DSG) moves the incident electron beam in a stepwise fashion over the specimen surface. If the signal received by the electron detector is close to background level (no image contrast), the computer selects the next $(x, y)$ coordinates in the scan sequence and moves the beam to the next picture point [9]. As illustrated in figure 4, when image contrast above a preset threshold is encountered (or below if adjusted for negative contrast) an auxiliary subroutine drives the beam in a preset pattern to determine the dimensions and shape of the feature (such as an inclusion particle).

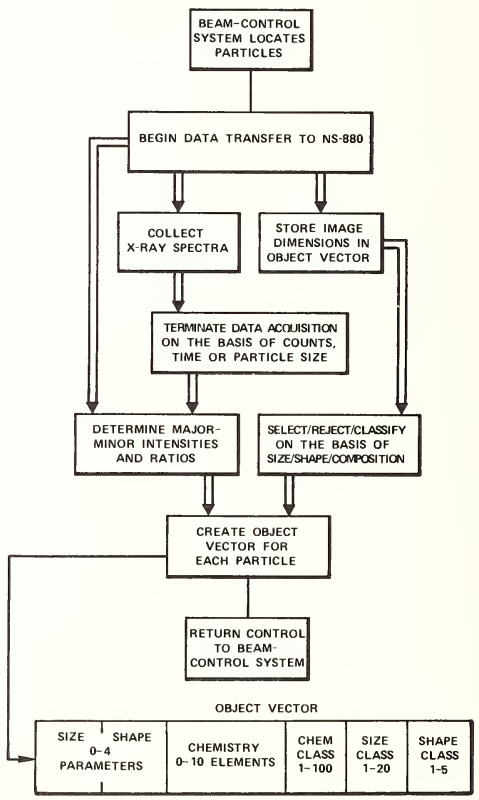

Figure 3. Block diagram of Flextran programs for computer-controlled size measurement, chemical analysis, and classification of image features. 


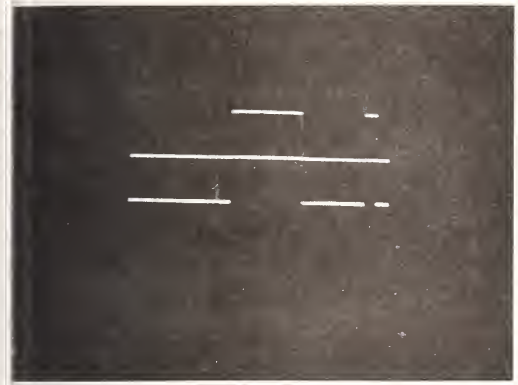

Figure 4. Illustration of use of threshold setting to trigger and terminate digital scan drive.

The preset pattern shown in figure 5 consists of four pairs of diagonals; each diagnol is terminated when the signal falls below the reference voltage. The pattern is repeated twice, once to locate the feature and once to determine the lengths of the diagonals hrough the centroid. The maximum, minimum, and average diagonals are stored; the centroid $s$ retained and used in a portion of a subroutine designed to prevent double counting.

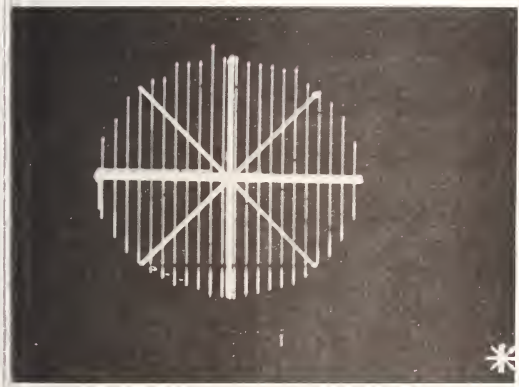

Figure 5. Illustration of preset pattern followed in AIA particle size and shape measurements.

As shown in figure 5, a low picture-point density is used in searching, thereby 17 lowing a large area to be analyzed per unit time. Simultaneously, the precision of the leasurement is maintained by using a higher point density (the on-point density) once a eature is located. This example is for an off-point density of 50 , and an on-point lensity of 200. Once the maximum, minimum, and average diagonals of the feature are determined and stored, the minicomputer returns to the search mode, or, alternatively, ositions the beam at the centroid of the feature and signals a second minicomputer to nitiate EDS $x$-ray analysis.

Beam-control programs are written in an interactive Flextran and supplied by LeMont cientific. One was written for particle-size measurement and plotting size-distribution fata. The second was written to measure the size and diameter of long straight features, a leasurement not satisfactorily performed with the preset-diagonal measurement.

Basically, the user specifies the magnification and the off-point and on-point lensities used in the search and analysis portions of the program, the portion of the image io be analyzed, and the type of picture-point matrix - $(n \times m)$, where $n$ specifies the 
horizontal off-point density, and $m$ specifies the vertical off-point density. At the end of each frame or scan of the image the operator can ask for a new frame, interim plots of the data, or new analyses.

The signals used for imaging, that is, the secondary (SE), backscattered (BSE), or transmitted (TSE) electron intensities can also be used for image analysis. The SE image, with a resolution of $\sim 100 \AA$, is useful for analyzing free particulates on a flat substrate. The TSE signal, with a resolution of $75 \AA$, is good for fine powders and air samples supported by a thin film of carbon. The BSE is especially useful for analysis of particles in a matrix or on a substrate which has a significantly different mean atomic number. The micrographs in figure 6 were taken with a BSE detector especially designed for AIA for a resolution of $200 \AA$ and show mixed $\mathrm{Al}_{2} \mathrm{O}_{3}$ and $\mathrm{MnS}-\mathrm{Al}_{2} \mathrm{O}_{3}$ inclusions in steel. In this case, AIA is carried out in two steps: first with a low threshold setting to measure the total inclusion content and size distribution, and then, with the higher setting as illustrated to restrict the analysis to "brighter" $\mathrm{Al}_{2} \mathrm{O}_{3}$ component.

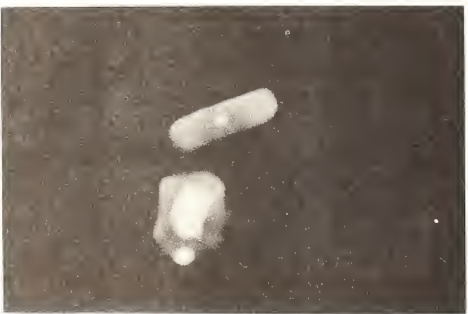

(a)
$10,000 \mathrm{x}$

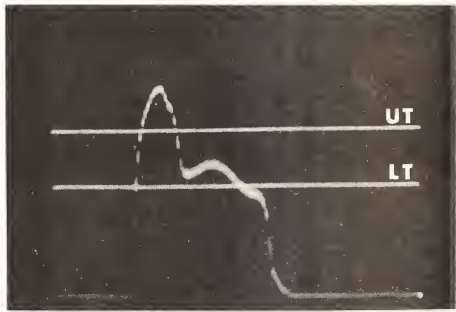

(b)

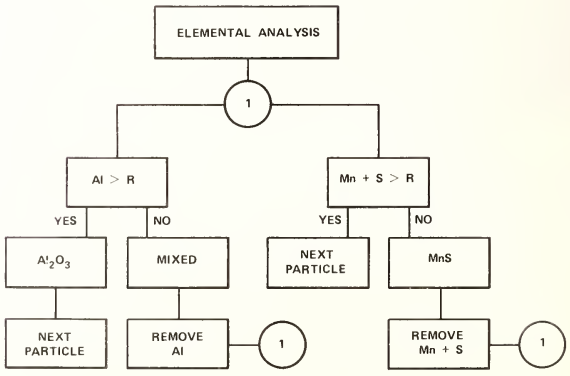

(c)

Figure 6. High resolution BSE micrographs. (a) $\mathrm{MnS}_{-} \mathrm{Al}_{2} \mathrm{O}_{3}$ inclusions; (b) monitor oscilloscope trace of BSE signal as the beam traversed a dual-phase inclusion. Extent of feature is defined by portion of the signal above lower threshold (LT) value; (c) block diagram of $x$-ray classification scheme. 
Measurements of high atomic number $(Z)$ particles on a low- $Z$ substrate made with any the three signals agree very well with each other within the limits of measurement by and ( $100 \mathrm{~A}$ at $1000 \mathrm{X}$ magnification). Measurements of $10 \mathrm{w}-\mathrm{Z}$ features in a high-Z matrix, uch as MnS particles in three percent Si steel, tend to be larger $(\sim 0.1 \mu \mathrm{m})$ when made with IE than when made with SE. This appears to be the result of edge effects and the greater epth from which the BSE signal is extracted.

The results of tests of the reproducibility of measurements made with AIA are ummarized in Table 1. A single MnS particle in a polished section of silicon steel was easured repeatedly at four magnifications. The mean and standard deviations were computed or the maximum diameter, minimum diameter, and average diameter. The accuracy, as epresented by the standard deviation, is seen to improve only slightly at higher magnificaion. This is an important result because it is more efficient to scan at low magnification , increase the number of particles encountered in a single field of view.

Table 1. Reproducibility of AIA measurements made on the same particle at four magnifications.

\begin{tabular}{|c|c|c|c|c|c|c|c|c|c|}
\hline \multirow[b]{2}{*}{$\begin{array}{l}\text { agnifi- } \\
\text { :ation }\end{array}$} & \multirow{2}{*}{$\begin{array}{c}\text { Number of } \\
\text { Measure- } \\
\text { ments }\end{array}$} & \multicolumn{2}{|c|}{$\begin{array}{c}\text { Average Diameter } \\
\mu \mathrm{m}\end{array}$} & \multicolumn{2}{|c|}{$\begin{array}{c}\text { Maximum Diameter } \\
\mu \mathrm{m}\end{array}$} & \multicolumn{2}{|c|}{ Aspect Ratio } & \multirow{2}{*}{$\begin{array}{l}\text { Maximum } \\
\text { Diameter } \\
\mu \mathrm{m} \\
\end{array}$} & \multirow{2}{*}{$\begin{array}{c}\text { Minimum } \\
\text { Diameter } \\
\mu \mathrm{m} \\
\end{array}$} \\
\hline & & Mean & $\begin{array}{l}\text { Standard } \\
\text { Deviation }\end{array}$ & Mean & $\begin{array}{l}\text { Standard } \\
\text { Deviation }\end{array}$ & Mean & $\begin{array}{l}\text { Standard } \\
\text { Deviation }\end{array}$ & & \\
\hline 1000 & 11 & 0.73 & 0.03 & 0.88 & 0.05 & 0.60 & 0.083 & 0.960 & 0.480 \\
\hline 2000 & 14 & 0.74 & 0.05 & 0.83 & 0.11 & 0.80 & 0.136 & 1.050 & 0.540 \\
\hline 4000 & 12 & 0.73 & 0.02 & 0.83 & 0.07 & 0.81 & 0.080 & 0.960 & 0.600 \\
\hline 8000 & 12 & 0.72 & 0.01 & 0.84 & 0.02 & 0.77 & 0.027 & 0.870 & 0.630 \\
\hline
\end{tabular}

\section{Illustrative Applications}

The numerous applications of SEM-AIA may be grouped into four general categories: ) single-inclusion type in a solid matrix for which very accurate size and distribution ita are of major interest (for example, MnS inclusions in stee1); (2) mixtures of iclusion types in a solid matrix, for which quantitative information about both the undance and the sizes of the various types are of primary interest (for example, mineral instituents in coal); (3) single-component powders, for which mean size and distribution 'e sought (for example, pigments); (4) multiple-component dusts, for which phase identificaon as well as size and abundance of the various components are required (for example, ispended particulates in air samples). An example of each of these categories is described , brief to illustrate salient features of AIA techniques.

Iclusions in Steels.

The size and nature of inclusions are important factors when interpreting or predicting le properties of steels or other metals. Although this subject has been investigated tensively by optical and electron microscope techniques, many problems require a level of curacy beyond that which can be achieved by conventional methods. For example, informaon about changes in size of inclusions in steels during prolonged heating at elevated mperatures is of considerable value for predicting the properties of finished steels as 11 as for basic studies of the growth mechanism. A typical computer printout of an A-SEM analysis of MnS inclusions in a three percent Si steel is shown in figure 7 . The ta can also be listed on a particle-by-particle basis if desired. 


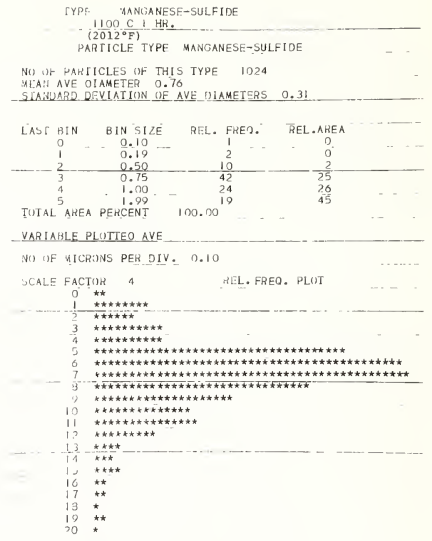

Figure 7. Example computer printout of an automatic image analysis of MnS inclusions in steel.

The results of analyses of a series of samples are presented in Table 2 and also plotted in figure 8 . The metallographic effects of heating time and temperature, although significant, are not large and could not be determined by conventional electron-microscope methods [10]. An example involving duplex $\mathrm{Al}_{2} \mathrm{O}_{3}-\mathrm{MnS}$ inclusions is shown in Table 3 . It is apparent that the effects of the special treatment could not be detected by conventional quantitative television microscopy, although they are quite evident by AIA-SEM analysis.

Table 2. Isothermal growth of MnS precipitates in three percent silicon steel as a function of annealing time and temperature.

Temperature

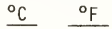

$850 \quad 1562$

$950 \quad 1742$

$1000 \quad 1832$

$1100 \quad 2012$
Average Diameter of MnS Particles, $\mu \mathrm{m}$

\begin{tabular}{|c|c|c|c|c|c|c|}
\hline \multicolumn{7}{|c|}{ Time, hours } \\
\hline $1 / 2$ & 1 & 4 & 16 & 24 & 64 & 240 \\
\hline - & 0.38 & 0.43 & 0.44 & - & 0.46 & 0.47 \\
\hline - & 0.43 & 0.45 & 0.49 & - & 0.48 & - \\
\hline - & 0.46 & 0.50 & 0.53 & - & 0.63 & - \\
\hline 0.76 & 0.76 & 0.80 & - & 0.82 & - & - \\
\hline
\end{tabular}




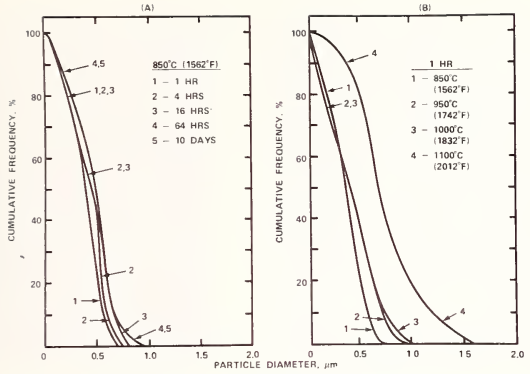

Figure 8. Illustration of AIA measurements of the growth of MnS inclusions in $\mathrm{Fe}-3$ percent $\mathrm{Si}$ steel.

Table 3. Weight percent $\mathrm{Al}_{2} \mathrm{O}_{3}$ and $\mathrm{MnS}$ in 4027 steels determined by automatic image analysis (AIA).

\begin{tabular}{|c|c|c|c|}
\hline & \multicolumn{2}{|c|}{ AIA } & \multirow[b]{2}{*}{ QTM } \\
\hline & $\mathrm{A}_{2} \mathrm{O}_{3}$ & $\mathrm{MnS}$ & \\
\hline Treated & 45.22 & 41.45 & $0.12-0.14$ \\
\hline Untreated & 77.30 & 11.82 & $0.12-0.14$ \\
\hline
\end{tabular}

ineral Constituents in Coal.

Most of the coal mined in the U. S. contains between 10 and 20 weight percent mineral atter. Some of this inorganic material can be removed by comminution and washing, but the emainder is so intimately associated with the coal macerals that its complete removal is ot feasible. This residual mineral matter affects the properties of coke after carbonizaion of the coal, and the nature of ash and slag formed during combustion. Detailed studies $f$ the mineral species, abundance, and size distribution of the included minerals in coal re needed to develop a basic understanding of their role in specific applications and ermit optimum use of various coals.

SEM-AIA has proven to be a valuable adjunct to other analytical techniques such as ptical petrography, chemical analysis, and Mossbauer spectroscopy, since it provides mineral dentification and size information on a particle-by-particle basis. Some 29 categories of ineral phases were established on the basis of the actually observed x-ray intensities of he 10 most common elements ( $\mathrm{Na}, \mathrm{Mg}, \mathrm{Al}, \mathrm{Si}, \mathrm{S}, \mathrm{Cl}, \mathrm{K}, \mathrm{Ca}, \mathrm{Ti}, \mathrm{Fe}$ ) found in coal ash. ifteen categories corresponded to common minerals, another nine represented mixtures of wo or more common minerals in which mineral grains formed a compound particle, and the emainder are various mixed but unidentified mineral constituents. A computer printout of he size and volume distribution of illite (clay) in a Pittsburgh Seam coal is shown in igure 9. A modification of the classical normative analysis scheme using chemical, ossbauer, and SEM-AIA data was used to interpret the results of all the analyses as preented in Table 4. Further details of this procedure can be found in the references ited $[11,12]$. 


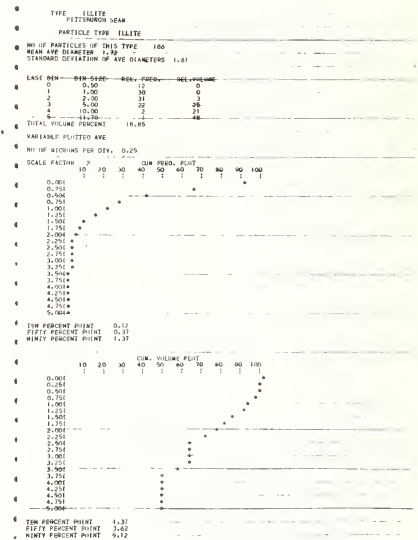

Figure 9. Computer printout of AIA analysis of a selected mineral constituent in a coal sample.

Table 4. AIA analysis of mineral constituents in coal.

\begin{tabular}{|c|c|c|c|c|c|c|c|c|}
\hline \multirow[b]{2}{*}{ Mineral } & \multicolumn{2}{|c|}{$\begin{array}{l}\text { Illinois } \\
\text { \#6 Seam }\end{array}$} & \multicolumn{2}{|c|}{$\begin{array}{c}\text { Pittsburgh } \\
\text { Seam, PA } \\
\end{array}$} & \multicolumn{2}{|c|}{$\begin{array}{c}\text { Pocahontas } \\
\text { Seam, WV } \\
\end{array}$} & \multicolumn{2}{|c|}{$\begin{array}{l}\text { Gholson } \\
\text { Seam, AL } \\
\end{array}$} \\
\hline & $\underline{\text { SEM }}$ & NORM & $\underline{S E M}$ & NORM & $\underline{\text { SEM }}$ & NORM & SEM & $\underline{\text { NORM }}$ \\
\hline Silicates & 83 & 75 & 59 & 76 & 87 & 74 & 26 & 27 \\
\hline Carbonates & 0 & 3 & 6 & 5 & 5 & 11 & 71 & 66 \\
\hline Pyrite & 15 & 15 & 26 & 19 & 2 & 3 & 2 & 7 \\
\hline Sulfates & 1 & 5 & 6 & ID & 2 & ID & $<1$ & $O D$ \\
\hline Other & 0 & 2 & 0 & 2 & 3 & $3^{\circ}$ & 0 & 1 \\
\hline
\end{tabular}

Single Component Powders.

Knowledge of the particle size distribution in fine powders produced by grinding or other processes for pigments, catalysts, or other application is frequently required for control or specification purposes. Sieving is commonly used to measure size distributions of particles greater than 40 micrometers in diameter, but a relatively large amount of sample material is required and the quality of sieves deteriorates with use so that the tolerances are degraded. SEM-AIA provides a very attractive alternative procedure.

Scanning electron micrographs of the -400 mesh and $+400-325$ mesh fractions of a dispersed industrial catalyst are shown in figure 10. Histographs of the diameters of these two fractions, shown in figure 11, are essentially normal distributions with mean 


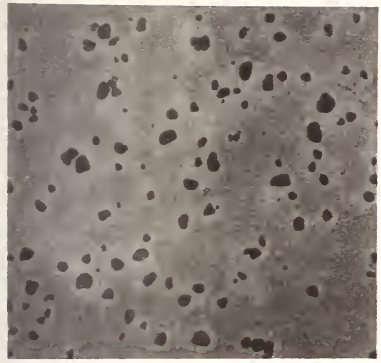

-400 Mesh

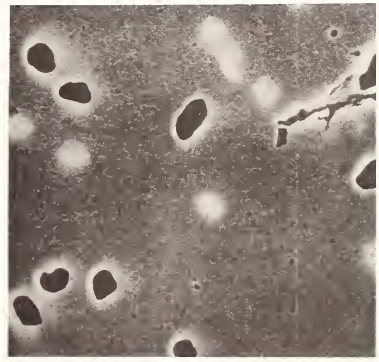

$+400-325$ Mesh

Figure 10. Typical micrographs of two size fractions obtained by sieving.
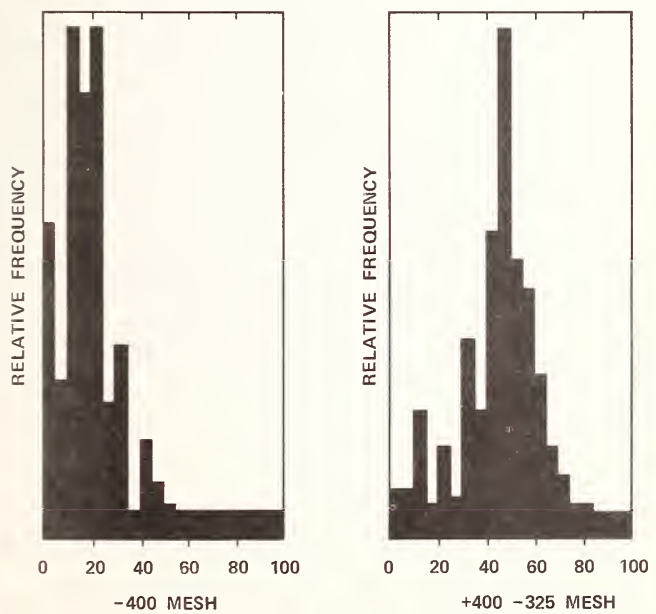

PARTICLE DIAMETER, $\mu \mathrm{m}$

Figure 11. Size distribution for two sieve fractions shown in figure 10. 
particle diameters approximately equal to the nominal sieve sizes, and 95 percent of th particles within \pm two standard deviations as expected. However, closer examination 0 results brings out several pertinent points relating to the accuracy of sieving techniques The weaving procedures used in the manufacture of screens cause the dimensions of the siev openings to vary somewhat, resulting in a range of openings in individual sieves and variation in average opening from sieve to sieve. ASTM specifications for U. S. standar test sieves in the SEM range allow a maximum variation of average opening of \pm 6 percent fo sieve sizes Nos. 80 and 100 , and \pm 7 percent for Nos. 200, 325, and 400 . In addition, not mor than 5 percent of the opening shall exceed the nominal opening by more than 30 percent fo the large sizes and 45 percent for the fine-mesh screens.

To check on the variability of a typical laboratory sieve, shadow "replicas" of th sieve were prepared by evaporating gold onto a carbon disk from a point source. A SE micrograph of the replica is shown in figure 12, and the deviations from square opening are quite evident. A cumulative frequency plot obtained by AIA on such a sieve replica i shown in figure 13. The maximum diameter of $67 \mu \mathrm{m}$ is close to specification; however, th fraction oversize, i.e., more than $57 \mu \mathrm{m}$, is appreciably greater than the 5 percen prescribed. It is clear that the accuracy of sieve testing could be improved appreciabl by calibrating each standard screen by AIA to determine calibration and quality parameters.

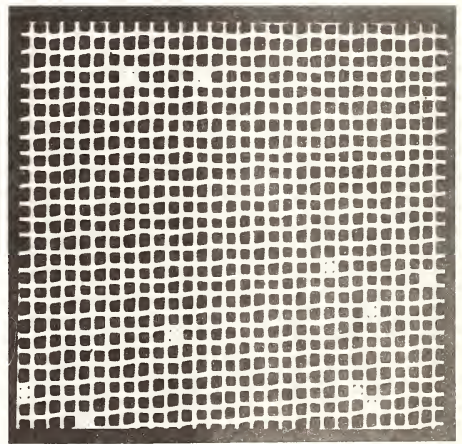

Figure 12. Scanning electron micrograph of a gold shadow "replica" of a used standard test sieve. 
A() OF MICKOUS PER DI\%. 3.n0

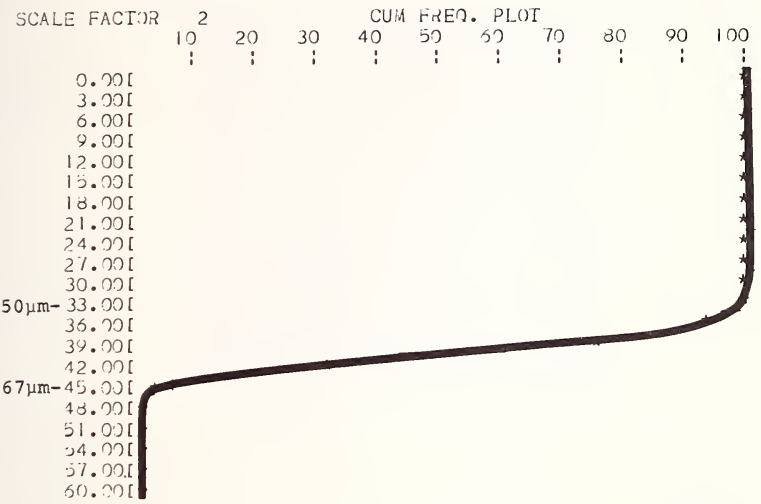

Figure 13. AIA derived cumulative frequency plot of opening size on a used standard test sieve.

The results of sieve and SEM-AIA analysis of a sample catalyst powder are summarized in Table 5. Some 3000 particles were analyzed by AIA, and to make a comparison on the basis of sieve sizes it was necessary to assign each to a specific sieve fraction according to ninimum diameter rather than average diameter (minimum and maximum diameter are determined oy AIA and average diameter is computed for each particle). Presumably the generally Illipsoidal particles pass through along their major axis.

Table 5. Comparison of size distribution of phthalic anhydride catalysts obtained by automatic image analysis and sieving.

\begin{tabular}{|c|c|c|c|c|c|}
\hline \multirow[b]{2}{*}{ Mesh } & \multirow{2}{*}{\multicolumn{3}{|c|}{ Opening Spec., $\mu \mathrm{m}$}} & \multicolumn{2}{|c|}{ Weight Percent } \\
\hline & & & & SEM & SIEVE \\
\hline-400 & $38,{ }^{a}$ & $48, b$ & $57^{c}$ & 2 & 3 \\
\hline-325 & 45 , & 57 , & 66 & 4 & 7 \\
\hline 200 & 75 & 91, & 103 & 8 & 3 \\
\hline-100 & 150, & 174, & 192 & 48 & 50 \\
\hline-80 & 180 & 207 , & 227 & 17 & 13 \\
\hline 60 & 250 & 283 & 306 & 21 & 20 \\
\hline
\end{tabular}

$\overline{\text { anominal size. }}$.

${ }^{b}$ Not to be exceeded by five percent of the openings.

Maximum. 
Particle sizing by SEM-AIA is limited to particles greater than about $0.1 \mu \mathrm{m}$ diameter and is not very satisfactory for particles much above $50 \mu \mathrm{m}$. Clearly, the technique is most useful for the small sizes for which sieving cannot be used and other available techniques are less straightforward. An example of determining a size distribution curve by combined SEM-AIA and use of a Sonic Sifter is shown in Table 6 . The -325 sieve fraction was analyzed by SEM-AIA and the +325 portion was sized by means of an Allen-Bradley model L3P Sonic Sifter. This device combines oscillating air and mechanical movement to determine sieve fractions methodically. As can be seen, significant differences occur within the fine particle fraction which would be difficult to observe by other techniques.

Table 6. Size measurements of catalyst particles.

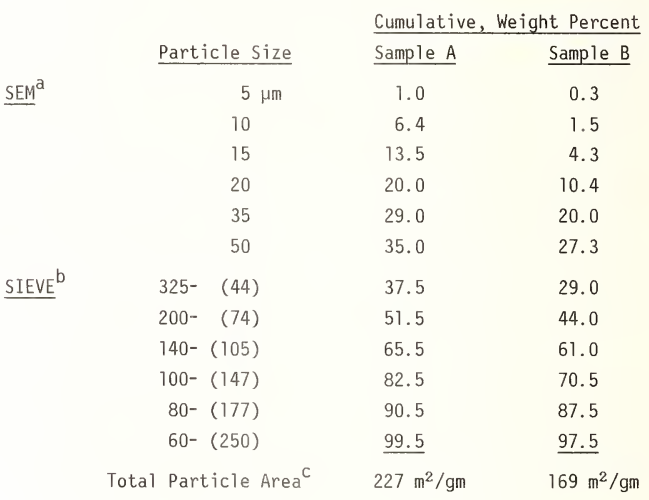

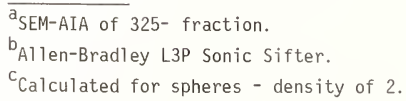

Suspended Particulates.

Analysis of particulates in air samples is an example of multiple-component dusts for which both particle identification and sizing are required. The sample mass is usually very small and a large number of different compounds are often present. SEM-AIA is ideal for this application, as $x$-ray spectroscopy can be used to classify particles according to the relative intensities of the elements they contain by computerized matching with a stored library of reference materials.

A SEM micrograph of a representative air sample from an exploratory study to evaluate the AIA technique is shown at low magnification in figure 14. Micrographs of individual particles and their x-ray spectra are shown in figure 15. The preliminary results of SEM-AIA analysis of several thousand particles are shown in Table 7 . When fully developed, more detailed studies with this technique will provide a means of identifying the contributions of various sources to the total suspended particulates in ambient air in industrial areas. 


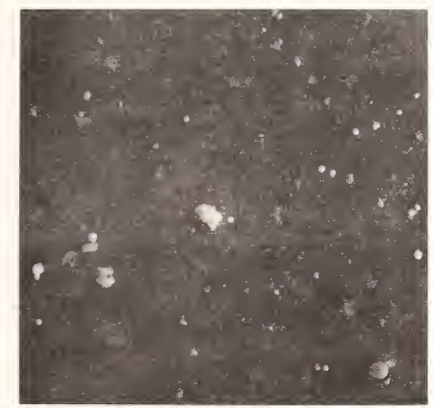

Figure 14. Low magnification scanning electron micrograph of a suspended particulate air sample. ( $800 \mathrm{X}$ )

Table 7. SEM-AIA analysis of air samples (wt. \%). ${ }^{a}$

\begin{tabular}{lcccc} 
Particle Type $^{b}$ & \multicolumn{2}{c}{ Location } & \\
\cline { 2 - 3 } Clays & 41 & 8 & Ambient \\
Slag & - & 60 & 27 \\
Fly Ash & 16 & - & 4 \\
Carbon & 33 & 2 & 5 \\
Fe-rich & & 6 & 30 & 2 \\
Misc. & 4 & - & 16
\end{tabular}

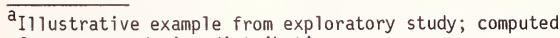
from measured size distribution.

${ }^{b}$ Determined from $x$-ray intensities of $\mathrm{Na}, \mathrm{Mg}, \mathrm{Al}, \mathrm{Si}$, $\mathrm{S}, \mathrm{Cl}, \mathrm{K}, \mathrm{Ca}, \mathrm{Ti}, \mathrm{Fe}$, and other metallic elements.

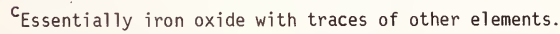




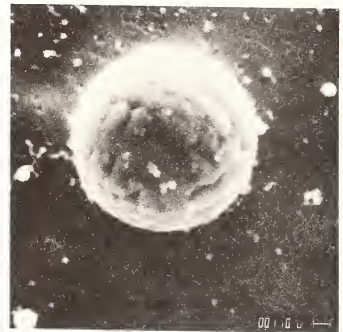

A) Iron Oxide (Steel)

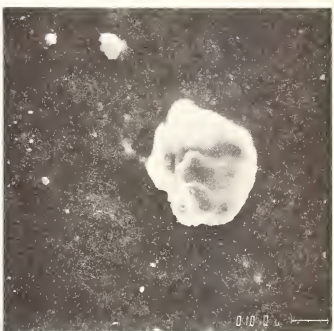

B) Clay (Road Dirt)

A,B) Secondary Electron Micrographs of Iron Oxide and IIIite Clay Particles.

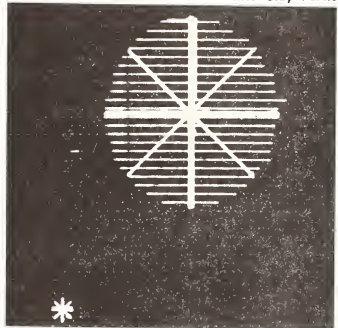

C) Digıtal Measurement

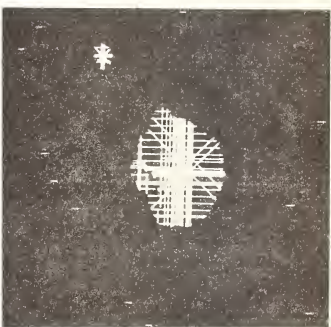

D) Digital Measurement

C,D) Digıtal Measurement of Particle Diameters.

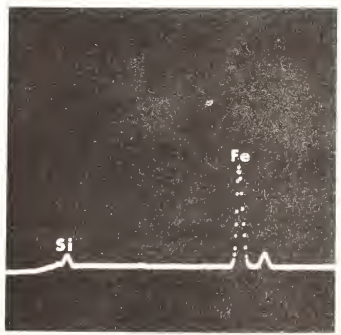

E) X-Ray Spectrum

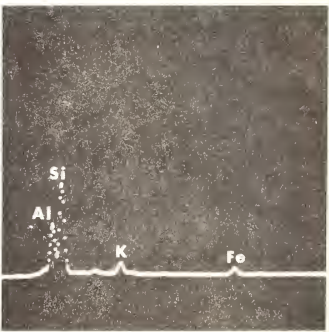

F) X-Ray Spectrum

E,F) X-Ray Spectra Used to Classify Particles by Composition.

Figure 15. Illustration of AIA size measurement and identification of particles in an air sample. 
Identification of particles by SEM-AIA is based on comparison of their $x$-ray spectra th spectra from standard compounds. As in other applications of electron-probe microalysis, the excitation and absorption of $x$-rays is affected by geometrical factors so that ie relative intensities of the various peaks depend on particle size and shape. Another surce of difficulty with particle identification is the similarity in composition which ten occurs with minerals and other complex phases, as shown in Table 8 . If positive lentification of very similar phases is demanded, then AIA must be supplemented by lected-area electron diffraction analysis [8]. This is a time-consuming operation and ans of automating the procedures are required.

Table 8. Dimensions and composition of particle "type" classes.

\begin{tabular}{|c|c|c|c|c|c|c|c|c|c|c|}
\hline \multirow[b]{2}{*}{ Type } & \multirow{2}{*}{$\begin{array}{l}\text { Average } \\
\text { Size, } \mu \mathrm{m}\end{array}$} & \multirow{2}{*}{$\begin{array}{l}\text { Aspect } \\
\text { Ratio } \\
\end{array}$} & \multicolumn{8}{|c|}{ Composition, ${ }^{\mathrm{a}}$ Percent } \\
\hline & & & $\underline{\mathrm{Na}}$ & $\underline{\mathrm{Mg}}$ & Al & $\underline{\text { Si }}$ & $\underline{K}$ & $\underline{\mathrm{Ca}}$ & $\underline{\mathrm{Ti}}$ & $\underline{\mathrm{Fe}}$ \\
\hline A (Serpentine) & 8 & 30 & - & 32 & - & 62 & - & - & - & 6 \\
\hline B (Pyroxene) & 5 & 5 & - & 16 & 4 & 60 & - & - & - & 20 \\
\hline C (Clay) & 7 & 19 & - & 1 & 40 & 50 & 1 & - & - & 8 \\
\hline O (Chlorite) & 5 & 7 & - & 12 & 28 & 38 & 3 & 1 & - & 19 \\
\hline$E$ (Mica) & 6 & 8 & - & 10 & 24 & 40 & 9 & 1 & 1 & 14 \\
\hline F (Silica) & 7 & 8 & 6 & - & 9 & 80 & 1 & 3 & 1 & 1 \\
\hline G (Augite) & 5 & 6 & - & 8 & - & 49 & - & 22 & - & 21 \\
\hline$H$ (Grunerite) & 5 & 7 & - & 11 & 2 & 61 & - & 1 & - & 26 \\
\hline
\end{tabular}

Detailed descriptions of the identification of microcrystals from single crystal ffraction patterns are available in texts and the research literature [13]. In brief, iterpretation of SAD patterns is accomplished by measuring two primary d spacings on the ittern (largest d spacing or smallest reciprocal lattice vectors) and the angle between Iem, as illustrated in figure 16. The measured spacings are compared by computer with 17culated interplanar spacings and angles from preselected known structures. Accurate llibration of the camera constant is essential, and this is usually achieved by vacuum lating the sample with a thin $f i 1 \mathrm{~m}$ of gold so that a standard pattern is superimposed on Ie $S A D$ image. Because of the greater penetrating power and higher resolution, it is Ivantageous to use the million volt microscope for SAD analysis.

A computer logic program, designated SEARCH, was developed to expedite comparison of Ie $S A D$ data with a large number of reference compounds [14]. It includes the effects of ittice symmetry. A typical set of data input statements required to run the SEARCH ogram is illustrated in figure 17. 


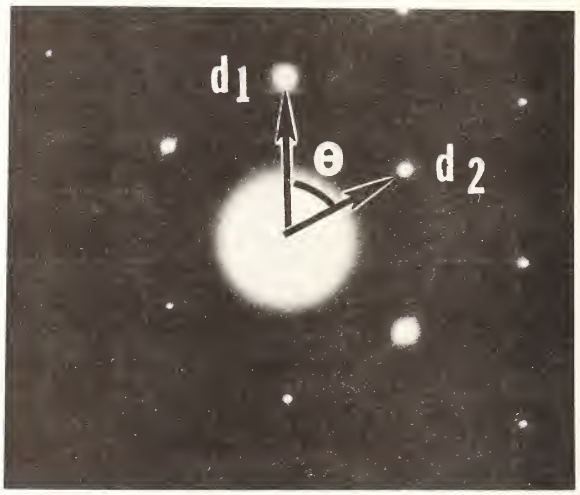

Figure 16. Illustration of basic measurements required to identify electron diffraction patterns.

\section{PROGRAM SEARCH}

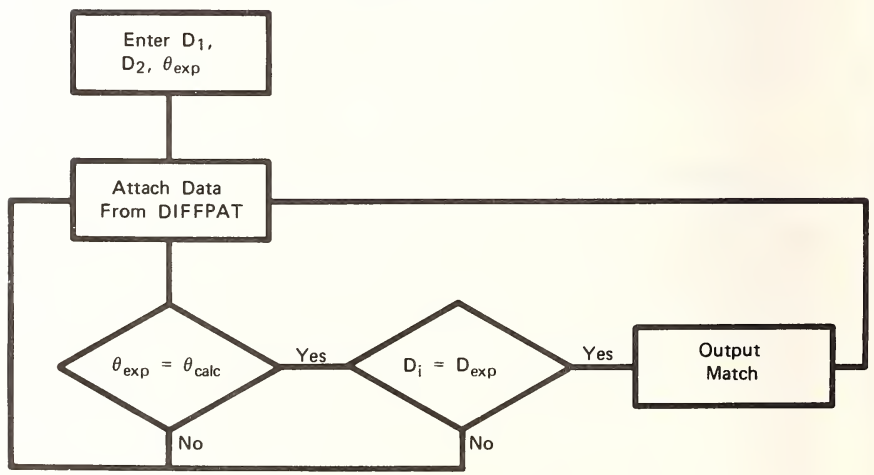

Figure 17. Computer logic designated SEARCH, to aid interpretation of electron-diffraction patterns. 
The combined use of $x$-ray spectrochemical and electron-diffraction methods, as illustrated in figure 18, is essential in the analysis of air samples. For example, the nisidentification of amphiboles and their erroneous association with asbestos has generated nuch confusion [15]. However, even with computer-associated pattern interpretation, it is just not practical to identify a large number of unknown particles by electron diffraction. A satisfactory compromise is to classify a large number of particles according to type classes on the basis of $x$-ray spectroscopy and aspect ratio by SEM-AIA [7]. Representative Jarticles in type classes are then positively identified by the electron-diffraction orocedures outlined above. This method introduces a statistical factor into particulate analysis, but does permit assignment of a probable error to the apparent concentration of zritical constituents, such as amphiboles, on a valid and verifiable basis.

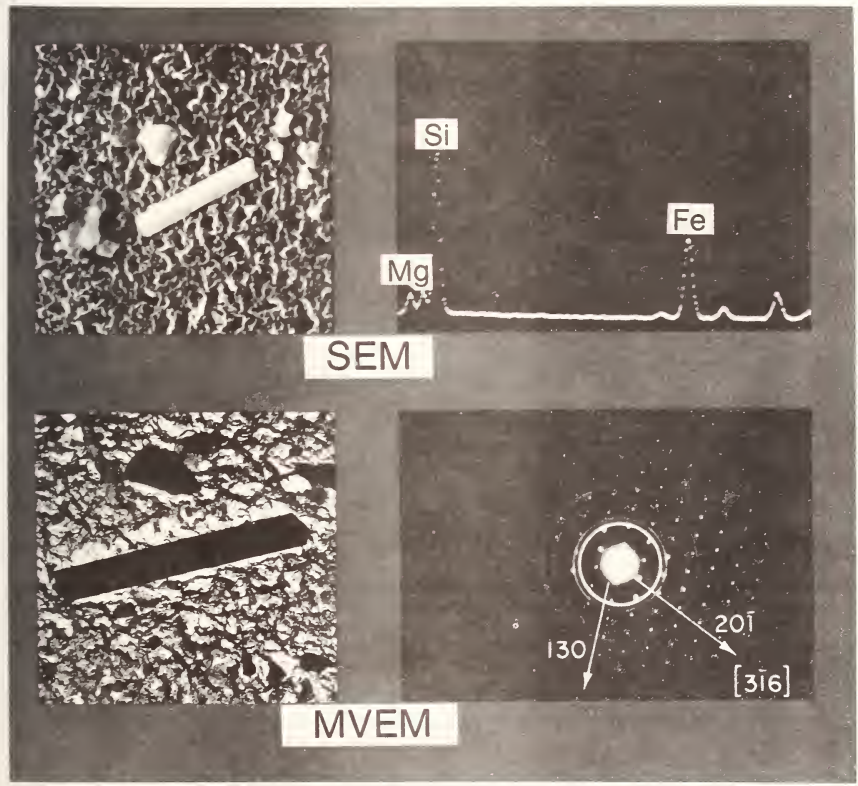

Figure 18. Illustration of combined use of AIA and SAD to identify particles. 
As illustrated by examples, AIA methods extend the capabilities of the SEM into the realm of quantitative characterization of particulates. Data can be obtained very rapidly, and no more than an hour is required to analyze several thousand particles and store the data for detailed interpretation by computer. The ability to determine statistically significant means, standard deviations, and other size-distribution parameters permits detection of small differences in characteristics that may show up as significant differences in bulk properties. Such data allow the microscopist to correlate his measurements with the results of conventional analytical procedures, or to select individual particles of special interest for more detailed microscopic studies. The tedium of repetitive measurements is eliminated, as well as subjective judgment by the operator. The investment in equipment and software development will be amply repaid by reduced analysis costs and more accurate and complete data.

Development of some of the techniques and illustrations described in this report (references cited) in collaboration with J. S. Lally, J. F. Kelly, A. Szirmae, S. Lentz, N. J. Panseri, G. M. Demchsin, Hsun Hu, G. P. Huffman, and F. E. Huggins is gratefully acknowledged.

\section{References}

[1] Isaacson, M. S. and Silcox, J., Report of a Workshop on Analytical Electron Microscopy, U1tramicroscopy, 2, 89 (1976).

[2] Silcox, J., Analytical Electron Microscopy Workshop Report, Cornell, July 1978.

[3] Oron, M. and Gilbert, D., "Combined SEM-Minicomputer System for Digital Image Processing," Proc. 9th Ann. SEM Symp., IITRI, Vol. 1, 1976, pp. 121-128.

[4] Ekelund, S. and Werlefors, T., "A System for the Quantitative Characterization of Microstructures by Combined Image Analysis and X-Ray Discrimination in the SEM," Proc. 9th Ann. SEM Symp., IITRI, Vo1. 1, 1976, pp. 419-424.

[5] Grant, G., Hall, J. S., Reid, A. F., and Zuiderwyk, M., "Multicompositional Particle Characterization Using the SEM-Microprobe," Proc. 9th Ann. SEM Symp., IITRI, Vol. 1, 1976, pp. 401-408.

[6] Hoover, M. R., White, E. W., Lebiedzik, J., and Johnson, Jr., G. G., "Automated Characterization of Particulates and Inclusions by Computer Controlled SEM/Probe," Proc. 10th Ann. Conf., Microbeam Anal. Soc., 1975, pp. 54A-54B.

[7] Lee, R. J., Lally, J. S., and Fisher, R. M., "Identification and Counting of Mineral Fragments," NBS Spec. Pub. 506, Workshop on Asbestos: Definitions and Measurement Methods, 1978, p. 387.

[8] Lee, R. J. and Fisher, R. M., "Identification of Fibrous and Non-Fibrous Amphiboles in the Electron Microscope," Annals of the New York Academy of Sciences, 1978, in press.

[9] Lebiedzik, J., "Multiple Electron Detector Method for Quantitative Microtopographic Characterization in the SEM," Doctoral Thesis, Penn State University, University Park, PA 16802, March 1975.

[10] Lee, R. J. and Hu, Hsun, "Automatic Image Analysis of MnS Precipitates in $3 \%$ Si Steel," 1978 , to be published.

[11] Lee, R. J., Huggins, F. E., and Huffman, G. P., "Correlated SEM-Mossbauer Studies of Coal Mineralogy," Scanning Electron Microscopy, I, 561-568 (1978). 
12] Huggins, F. E. and Huffman, G. P., "Mossbauer Analysis of the Fe-Bearing Phases in Coal, Coke, and Ash," Rev. Chapter to be published in Analytical Methods for Coal and Coke, Vol. 3, ed. Clarence Karr, Academic Press.

13] Lee, R. J., "Basic Concepts of Electron Diffraction and Asbestos Identification Using Selected Area Diffraction. Part I: Current Methods of Asbestos Identification Using SAD. Part II: Single Crystal and SAD," Proc. Scanning Electron Microscopy, 1978, Vol. 1, SEM, Inc., 1978.

14] Lally, J. S. and Lee, R. J., "Computer Indexing of Electron Diffraction Patterns Including the Effect of Lattice Symmetry," Proc. EMSA, 1977, p. 22.

15] Gravatt, C. C., Lafleur, P. D., and Heinrich, K. F. J., NBS Spec. Pub. 506, Proc. of Workshop on Asbestos: Definitions and Measurement Methods," November 1978. 
lational Bureau of Standards Special Publication 533. This Publication Originated from a pecial Session on Particle Analysis, 13th Annual Conference of the Microbeam Analysis ociety, Ann Arbor, MI, June 22, 1978. (Issued A.pri1 1980)

CHARACTERIZATION OF COAL GASIFICATION PARTICULATES BY SEM, EDS, AES, XPS, AND ITS RELEVANCE TO INHALATION TOXICOLOGY

D. L. Davidson and E. M. Gause

Southwest Research Center

P. 0. Drawer 28510

San Antonio, Texas 78284

\section{Introduction}

The initial site of deposition of an inhaled particle depends primarily upon its size; articles larger than $10 \mu \mathrm{m}$ in diameter are trapped in the nose, while particles etween 10 and $3 \mu \mathrm{m}$ are deposited in the upper respiratory tract, and particles elow $3 \mu \mathrm{m}$ reach the deep lung or alveolar region before being deposited. There re some exceptions to these generalizations as very long, narrow asbestos fibers have been ound in the deep lung upon autopsy $[1]^{1}$. Particles smaller than $3 \mu \mathrm{m}$ are generally eferred to as being in the respirable size range.

Once inspired particles are deposited in the deep lung, clearance mechanisms involve hagocytosis by alveolar macrophages or solubilization by lung fluids. In the phagocytic rocess, particles are ingested by alveolar macrophages which then attempt to degrade the articles enzymatically, figure 1. If there are nonpolar organic compounds present on the

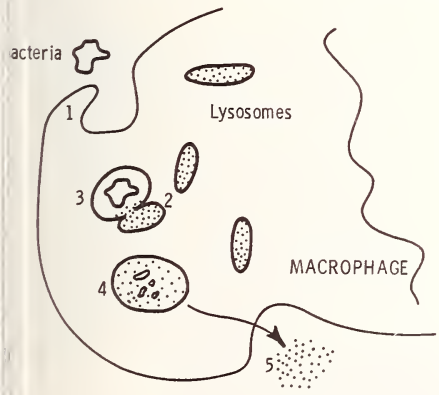

Figure 1. Schematic representation of the process of macrophage phagocytosis: (1) Ingestion of the bacteria, (2) Lysosomes containing digestive enzymes, (3) Release of the enzymes into the phagosome surrounding the bacteria, (4) Digestion, (5) Elimination.

irfaces of the particles, they may be converted into aqueous-soluble forms by the action monooxygenases (mixed function oxidases) in preparation for excretion in urine; the ater-soluble forms may be more or less toxic than the original form depending upon the ature of the starting material - i.e., metabolites produced may be mutagenic or carcinonic. Metallic and organo-metallic species adsorbed on particle surfaces may be removed ither intracellularly or extracellularly by binding to proteins or dissolution in lung luids; toxicity will depend upon the specific metals and oxidation states of the metals 
involved. After removal of substances adsorbed on particle surfaces, the composition of the residual particle may be primarily inorganic salt or mineral, or graphitic carbon; however, if the residue is insoluble in lung fluids, the only mechanism by which it can be cleared is phagocytosis and transport out of the lung by alveolar macrophages. Since the macrophage will not be able to digest particle residue in the same way it degrades ingested microorganisms, through the action of lysosomal hydrolytic enzymes, the undigested particle may be released to be taken up by another macrophage in a futile cycle; the release of the particle (exocytosis) will be accompanied by the release of hydrolytic enzymes which can damage lung tissue, figure 2. Alternatively, if substances adsorbed on surfaces of the particles, or

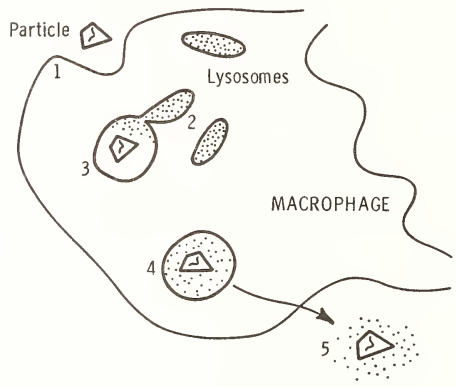

Figure 2. Schematic representation of what may occur when a macrophage cannot phagocytize a particle: (1) Ingestion, (2) Lysosomes containing digestive enzymes, (3) Which are released into the phagosome, (4) Digestion cannot proceed, (5) The particle and the enzymes are eliminated.

or the particles themselves (e.g., silica), are toxic to macrophages, they will cause death of the macrophages resulting in cell lysis and release of both hydrolytic enzymes and the toxic particles which can then be taken up by another macrophage to repeat the cycle. This effect can result in foci of dead and dying macrophages - an observation which has been linked to tumor formation and to tissue necrosis [2]; it will also result in local concentrations of the phagocytized toxic particles or "hot spots." There is evidence that particles which cannot be degraded by alveolar macrophages are eventually cleared from the lung only by dissolution in lung fluids and transport into the blood system - a process which may take years, or may never be successful, depending upon the solubility properties of the particle.

Toxic chemical species can be concentrated on the surfaces of respirable particles by condensation and adsorption during the cooling of combustion products, resulting in much higher biologically available levels of such species than would be anticipated from bulk particle analysis [3]. In fact, high surface concentrations of species not even detectable by conventional bulk analysis or by single particle $x$-ray microprobe analysis can be attained. This surface form of concentration also maximizes biological uptake and biological impact of the toxic species, in that leaching by biological fluids is facilitated and contact of cells with the surface-bound species is maximal. Surface deposition also results in a greater concentration of toxic trace elements the smaller the particle diameter; and the smaller the particle diameter, the more likely it is to be inhaled and deposited within the deep lung [3].

Chemical nature, oxidation state and concentration of species bound to the surface of respirable particles are determined by the inorganic components of fuels which may be present in extremely low concentrations and by the conditions of the combustion process. The combustion process may result in condensation of minor fuel components upon surfaces of effluent particles leading to relatively high "environmentally effective concentrations." The capacity of particles resulting from a given process for producing serious adverse health effects is presently not understood, but is growing in importance as energy technology switches to alternate fuel sources, both stationary and mobile. 
Particles described in this study were obtained from a European coal gasification "lant. Details of the particle collection procedure are unknown. The particles were sieved o separate particles in the respirable size range from the bulk sample, and only the fracFion below $20 \mu \mathrm{m}$ in diameter was examined. The larger size fractions were ground in a ball :ill with alumina grinding balls and re-sieved. The fraction of this ground material below $0 \mu \mathrm{m}$ in diameter was also examined by surface analysis techniques.

For examination, a sample of particles was placed into reagent grade isopropyl alcohol, nd this slurry was then flooded onto sintered silver filters (Selas Co.) having a pore iameter of $0.45 \mu \mathrm{m}$.

\section{Bulk Analysis}

Because of the small size of the particles, electron penetration at the working voltges necessary for $x$-ray microprobe analysis causes fluorescence from most parts of the article volume, as opposed to surface analysis by electron spectroscopy. The $x$-ray nalysis was carried out at the University of Texas Health Science Center in San Antonio sing a JSM-35 Scanning Electron Microscope (SEM) equipped with a Tracor Northern model 880 tergy dispersive $x$-ray spectrometer. Numerous particles were individually analyzed and "s hotographed. The photographs of three typical particles are shown in figure 3 , and the - ray spectroscopy of these particles is shown in figure 4. The physical appearance of the leilver filter may also be seen in the photographs, and the chemical composition of the aliter is shown in figure 5. From these illustrations and data taken from a number of ther particles, the particles were found to contain principally aluminum, calcium, iron, agnesium, silicon, sulfur, and perhaps small amounts of titanium or barium, chlorine and opper. The particles shown in figures $3 a$ and $3 b$ have sufficient quantitites of magnesium $r$ aluminum, silicon, and iron that they might be considered to be in the asbestos family $f$ minerals. A quantitative analysis of the particle shown in figure $3 b$ indicated the onstituents shown in Table 1 (excluding the silver filter).
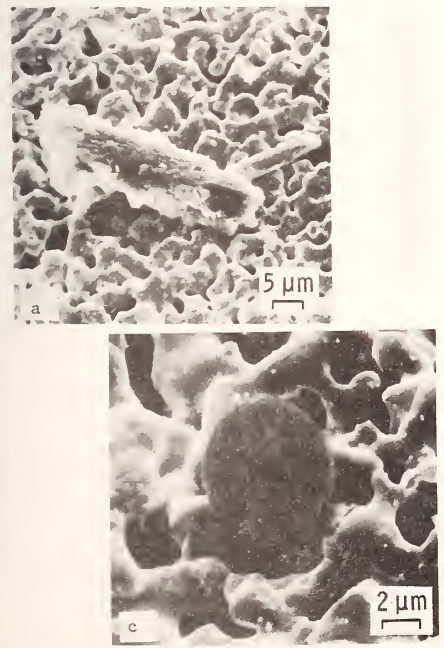

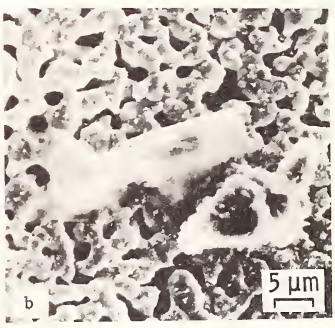

Figure 3. SEM micrographs of three of the particles. 


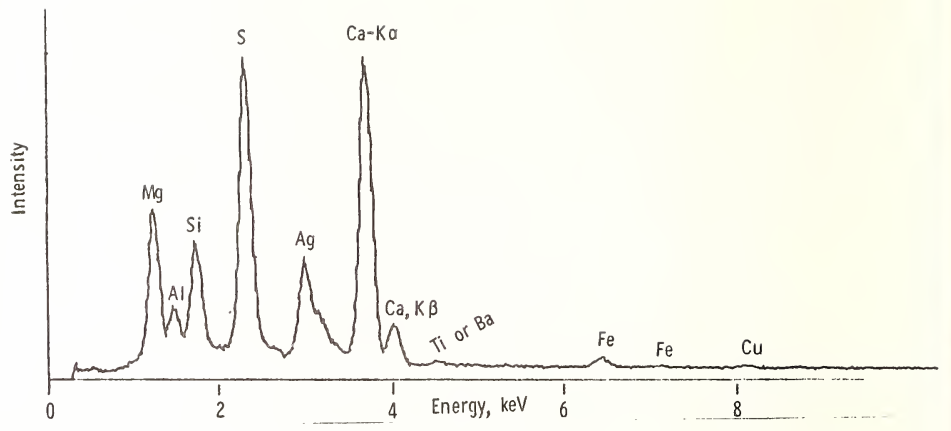

Figure $4 a$.

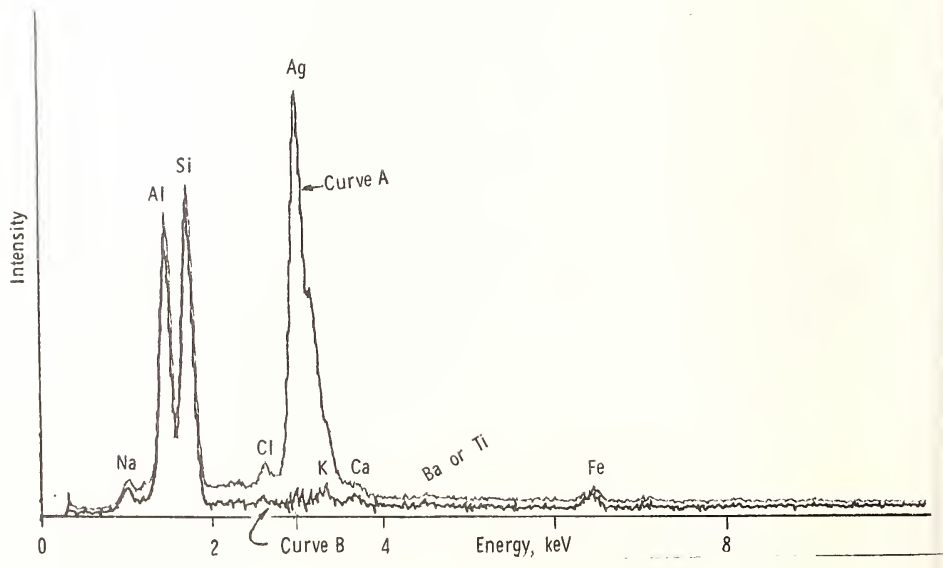

Figure 4b. 


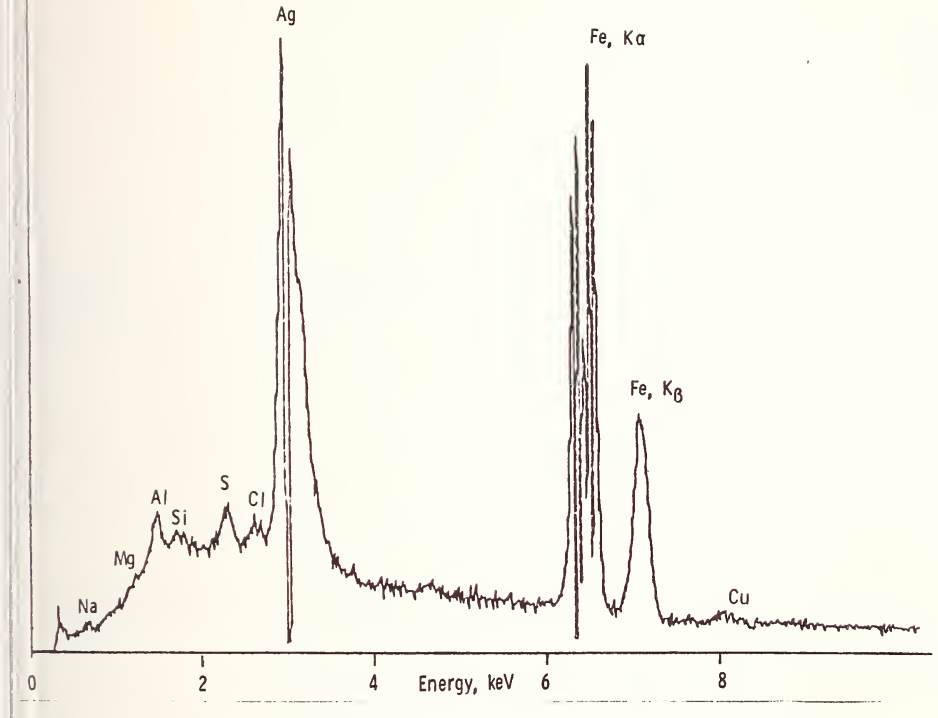

Figure $4 c$.

Figure $4 a, b$, and $c$. Energy spectrum of the three particles shown in figure 3 showing their bulk elemental chemistry. 


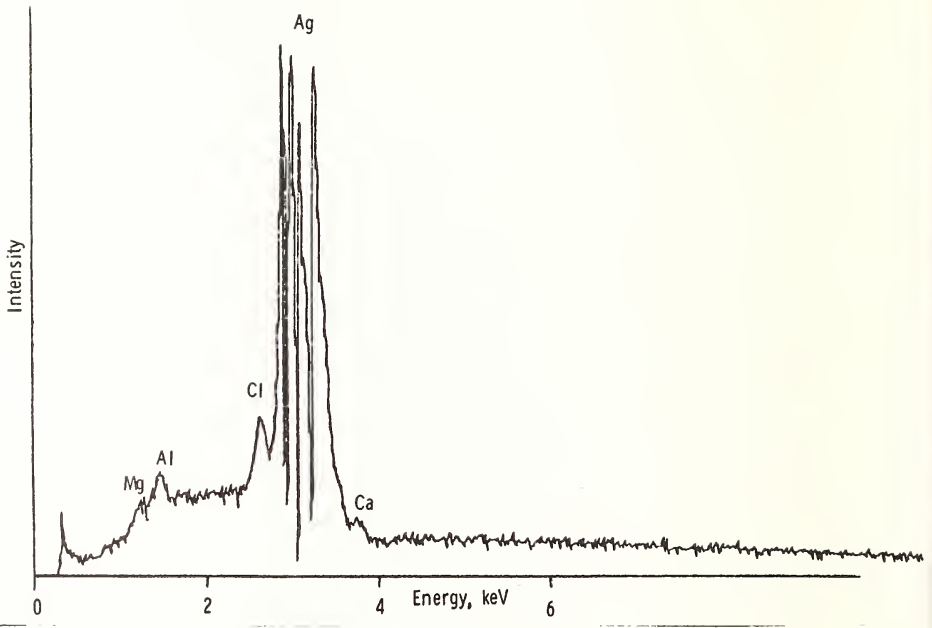

Figure 5. Energy spectrum of the silver filter carrier for the particles.

Table 1

\section{Element}

A1

Si

$\mathrm{Fe}$

$\mathrm{Na}$

Cl

\section{Concentration (\%)}

31.8

30.6

3.5

14. 1

13.9

The techniques used in this analysis were the standard background subtraction and ZAF correction techniques furnished by the equipment manufacturer. The curve with silver filter spectrum subtracted is shown in figure $4 b$ as curve $B$. 


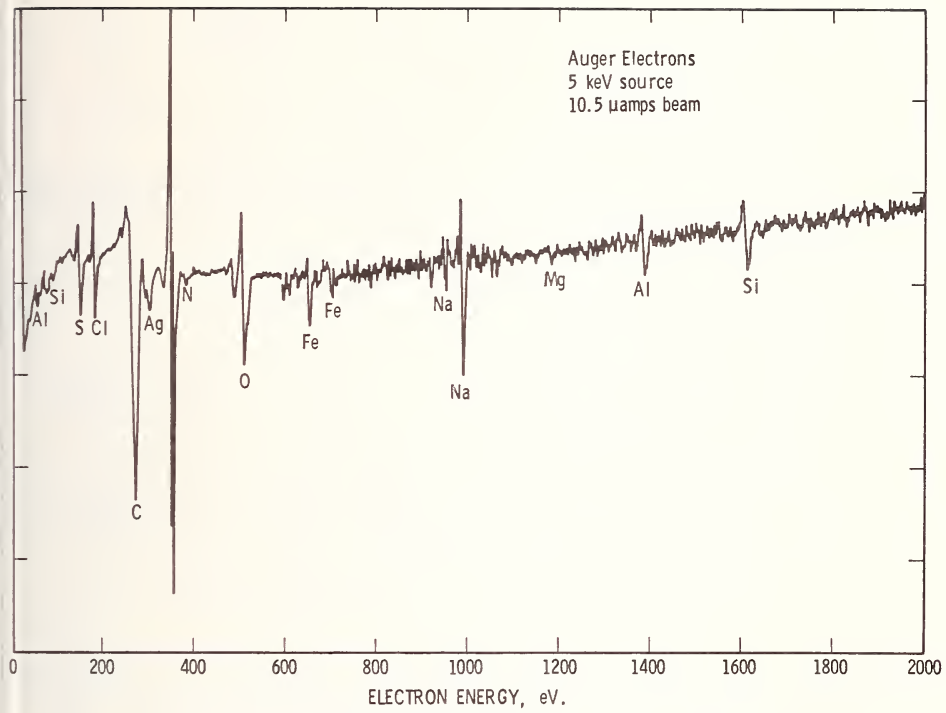

Figure 6. Auger electron spectrum of the unground particles, showing their surface chemistry.

\section{Surface Analysis}

The same particulate material (but samples different than those examined by energy ipersive $x$-ray analysis) was also subjected to surface analys is by Auger Electron Spectroupy (AES) and x-ray Photoelectron Spectroscopy (XPS), also called ESCA. Because both of ese techniques [4] depend on the energy spectrum of relatively low energy electrons, only face and near-surface material is included in the analysis, as opposed to the larger lume of material from which $x$-rays originate in the microprobe analysis. Equipment lufactured by Physical Electronics Industries was used for both the AES and XPS analyses.

The Auger electron spectra of the particulate material, figure 6 , and of the ground 1 sieved material, figure 7 , show that the unground material has a greater surface conitration of constituent elements than does the ground material, which has a higher conitration of carbon. As with the bulk analyses, aluminum, magnesium, and silicon are ain prominent elements, although it is not possible to know if they are in the correct icentrations to be considered as any of the minerals constituting asbestos. The presence sulfur in both spectra is also considered significant, even though the concentration is 


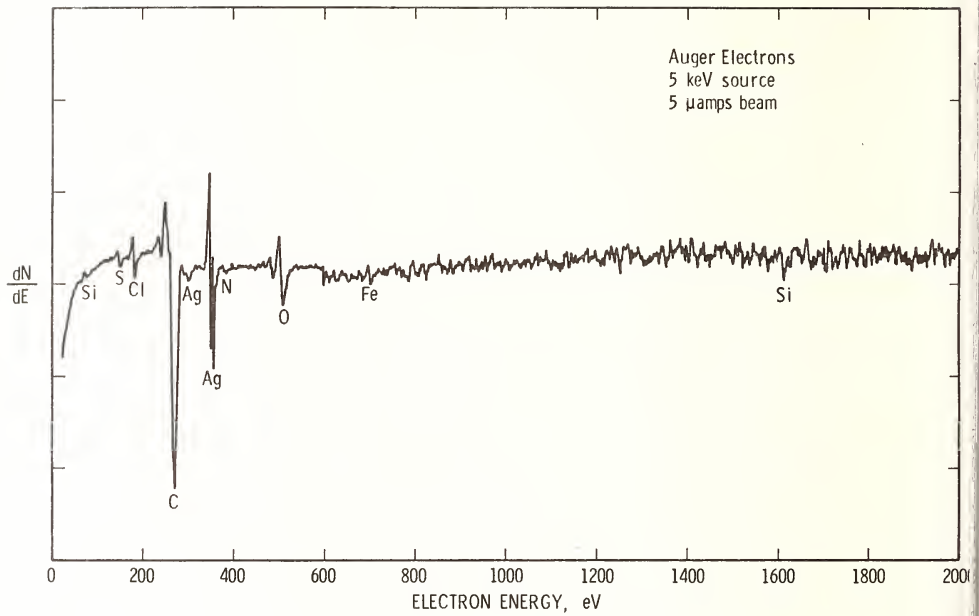

Figure 7. Auger electron spectrum of the ground particulates.

The $x$-ray photoelectron spectra of the unground and ground particulates are shown in figures 8 and 9 . The most significant finding from these spectra is the presence of the heavy metals, lead, selenium, and molybdenum, on the surface of the unground material; lead is indicated to be in fairly high concentration. For the ground material there is comparatively a higher concentration of carbon and less of the other significant elements. In this spectra, the presence of a small nitrogen peak is clearly indicated, while it was not clearly found for the underground material; whereas there may, in fact, be a nitrogen peak in the unground material spectra, the concentration is very low compared to that for the ground material. 


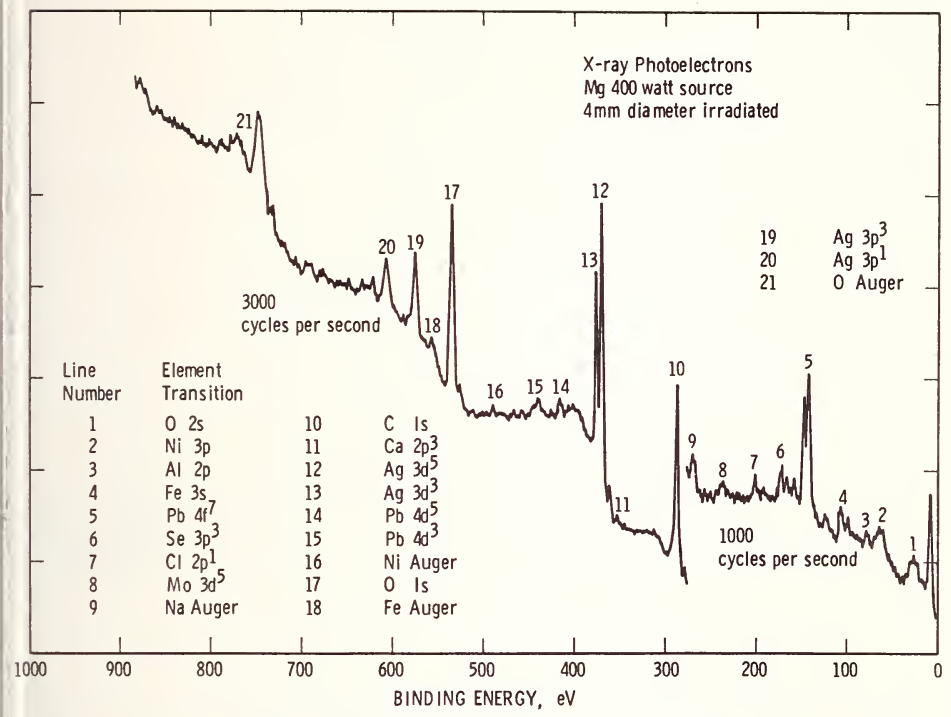

Figure 8 . X-ray photoelectron spectrum of the unground particles. 
X-ray Photoelectron

SPECTROSCOPY

GRAPHITE

\author{
Carbon Peak
}

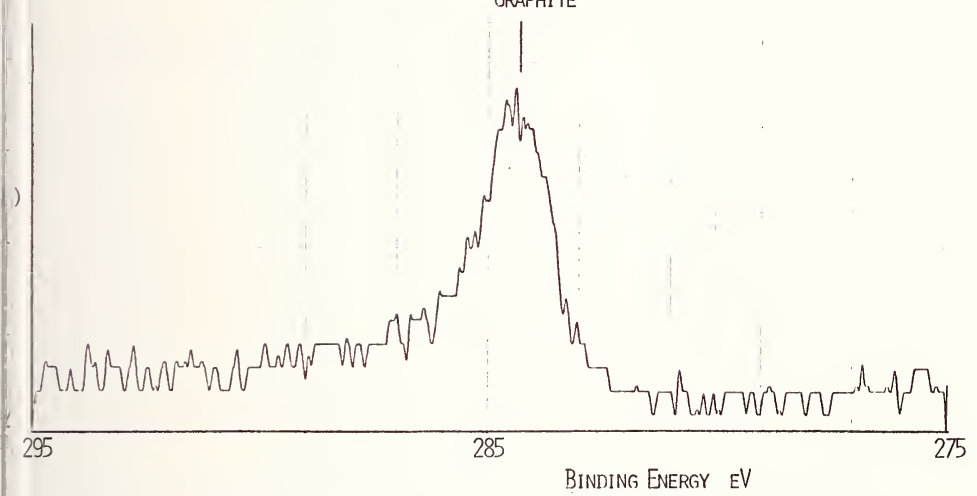

Figure 10. X-ray photoelectron spectrum of the unground carbon is peak. 


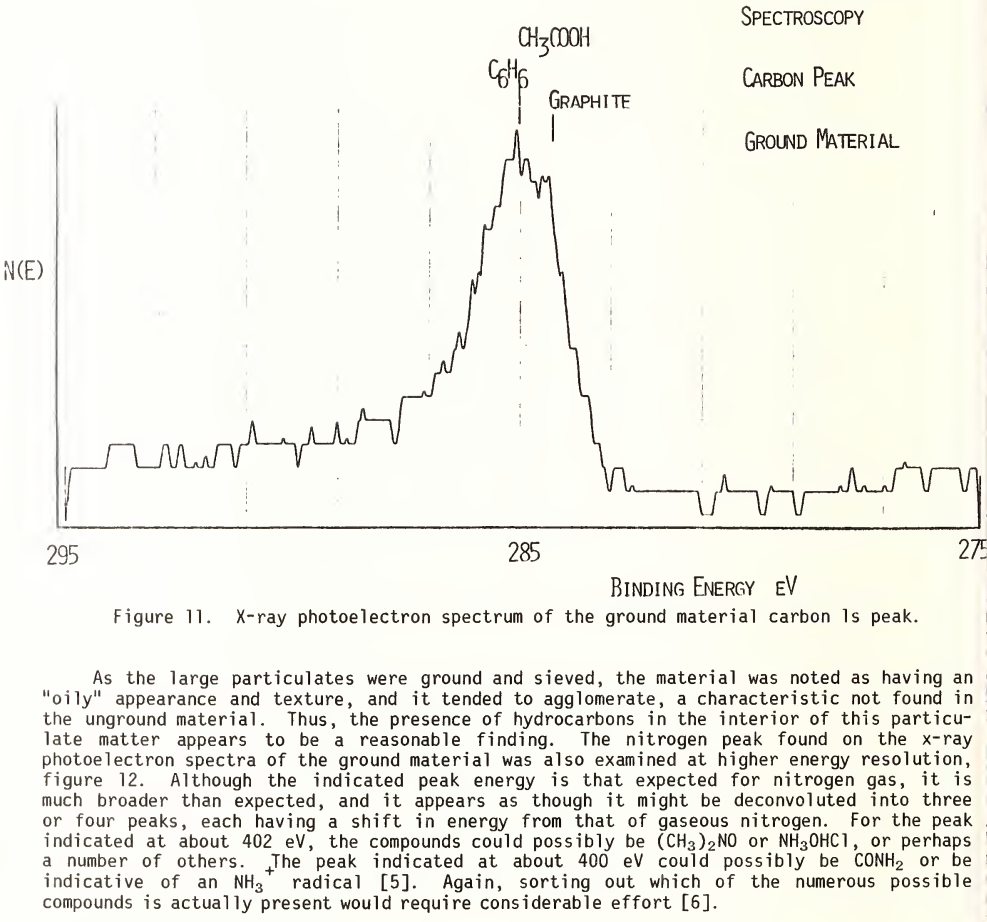

$X$-ray PHOtOElECtRoN SPECTROSCOPY

Carbon Peak

ground Material

Binding Energy eV 


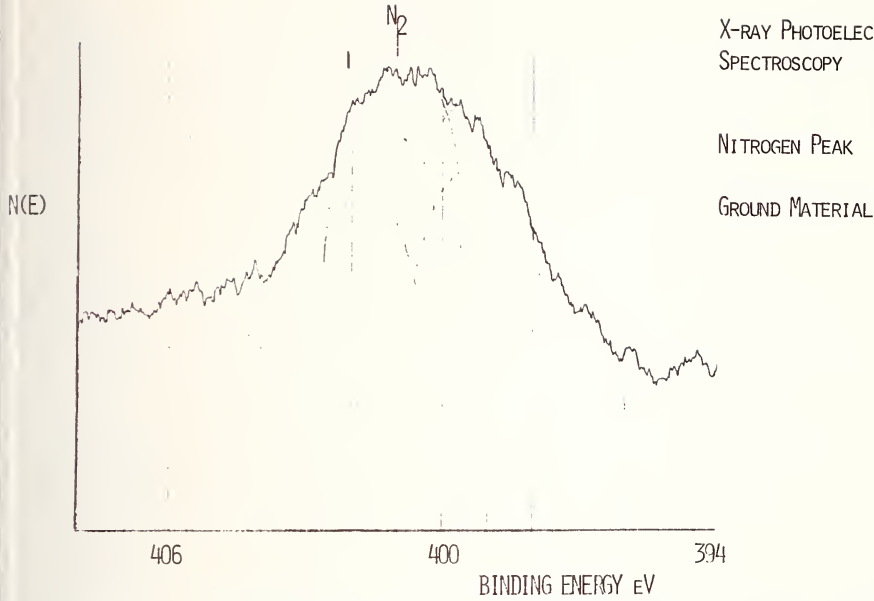

Figure 12. X-ray photoelectron spectrum of the ground material nitrogen is peak.

The Auger electron spectra of the ground material was examined after removal of approximately $100 \AA$ of material by argon ion bombardment (sputtering). The aluminum, iron, magnesium, and silicon peaks, found to be largely suppressed as compared to their amplitude for the unground material, were found to again be present in small concentrations, and the carbon peak was somewhat attenuated in comparison to the unsputtered peak. This finding appears to indicate that there is a thin covering of carbon, possibly in the form of hydrocarbons, on the surface of the ground material, which would correlate well with the XPS and visual observations.

\section{Discussion}

Results of the present study indicate that there is a large degree of variability in bulk composition and physical properties between individual particles originating from a common source, suggesting that careful attention must be given to obtaining representative samples. While the surface analysis techniques employed here do not allow examination of individual particles, it is likely that the observed differences in particle bulk composition are reflected in the nature of chemical species adsorbed on the surfaces of the particles due to variations in temperature of volatilization and condensation, position within the stack at which particle cooling allows condensation/adsorption onto surfaces, reducing or oxidizing atmospheres, etc.

Surface anlaysis revealed the presence of lead and selenium, both of which have wel1documented toxicities associated with inhalation exposure [7]. Lead and selenium exhibit neurotoxicity with central nervous system involvement in addition to causing damage to various other organs such as kidney, liver, and spleen; selenium is also considered to be embryotoxic and teratogenic. The observation that the concentration of these two elements was greatly attenuated after grinding of the particles offers additional proof of their surface localization and concentration. 
In contrast to lead and selenium which were found only on the particulate surfaces, sulfur was observed both on the surface of the ground and unground particles and in bulk by $x$-ray fluorescence, indicating that it is present throughout the particles. Although it was not determined in these studies, the chemical form of sulfur could be quite significant with respect to potential toxicities of the particles. Other surface changes observed after grinding of the particles were: (1) a shift in the position of carbon peak indicating the presence of hydrocarbons, and (2) surface exposure of reduced forms of nitrogen, some of which were observed to be chemically bound to other elements. The association of combined nitrogen and hydrocarbon-like carbon may well reflect the occurrence of organic nitrogencontaining compounds which would be expected to result from the high temperatures of the coal gasification process. Many organic nitrogen-containing compounds such as aromatic amines have been found to be carcinogenic or mutagenic [8].

Both bulk and surface analyses indicated the presence of elements comprising the various minerals generically known as asbestos although the actual existence of these minerals in samples examined was not verified. Asbestos is, of course, capable of producing grave pulmonary disease, in that asbestos fibers too long to be ingested by macrophages cause collagen production and fibrogenesis or asbestosis; asbestos can also cause lung cancer [9].

Inhalation of particulates is a direct route of entry into the body. It has been reported that approximately 25 percent of inhaled particles in the respirable size range settle in lung tissues, a similar amount is exhaled, and the remaining 50 percent is diverted to the pharynx, where it is swallowed [10]. Combustion-derived particulates apparently possess a variety of chemical species condensed onto or adsorbed on their surfaces, including trace metals, organic compounds, and ionic species. Alveolar absorptive efficiency for most trace elements is 50-80 [3] and efficiency of absorption in the stomach is 5-15 percent [11]. Many trace elements are carcinogenic, mutagenic, cytotoxic, or can disturb vital biochemical equilibria causing a variety of toxicological manifestations. Organic compounds are either metabolized by phagocytic cells or solubilized by binding to proteins and transported to other sites; many representatives of this class of compounds are either cytotoxic (causing cell death), or carcinogenic and therefore capable of causing cancer of the lung or of other organs upon transport to distal sites in the body. The processes of carcinogenesis and mutagenesis are characterized by very long lag times (decades) between exposure and appearance of effect; there processes may also be initiated by extremely small amounts of material, so that low doses of inhaled particulates may be responsible for cancers or other irreversible toxicities appearing many years later.

The presence of several toxic metal species on the suifaces of the particles described herein and exposure of other potentially toxic material upon grinding of the particles, indicating that these substances could be gradually released or extracted by tissues, illustrate the need for careful consideration and quantification of the concentrations and distributions of these surface-bound species and of tissue residence times and bioavailability of occult bulk species within a representative sample of respirable particulates. This information could then be employed for estimation of extent of exposure; and when relatistic information as to atmospheric concentration becomes available, and when doseresponse data is obtained from animal exposure studies, it will be possible to estimate risks involved in human inhalation exposures.

\section{Summary and Conclusions}

Surface analytical techniques (Auger electron and x-ray photoelectron spectroscopies) have found biologically important chemical species not detected by bulk ( $x$-ray microprobe) analysis.

From consideration of the chemical nature of surface-bound species, together with their concentration and biological accessibility, there is reason to believe that, for most types of respirable particles, the chemical species found on their surfaces may be more significant with respect to potential toxicological effects than the particles themselves. 
This work was supported in part by the U. S. Environmental Protection Agency under ontract No. 68-02-2286 MDD2. We very much appreciate access to the Health Science Center EM Laboratory equipment, and the help of Dr. Nancy Smith of that Laboratory. The assisance of Carl Hildebrand of Physical Electronics Industries in arranging access to the AES nd XPS surface analysis equipment is a'so gratefully acknowledged. A useful discussion ith John Funnell, Institute Scientist, is also acknowledged.

\section{References}

[1] Timbrell, V., Inhalation and Biological Effects of Asbestos, in Assessment of Airborne Particles, T. T. Mercer, et al., editors, Thomas Publishers, Springfield, IL, (1972) pp. $429-441$.

[2] Mohr, U., Reznik-Schuller, H., Reznik, G., Grimmer, G., and Misfeld, J., Investigations on the Carcinogenic Burden by Air Pollution in Man. XIV. Effects of Automobile Exhaust Condensate on the Syrian Golden Hamster Lung, Zbl. Bakt. Hyg., I. Abt. Orig. B $, \underline{163}, 425-432$ (1976).

[3] Natusch, D. F. S., Wallace, J. R., and Evans, C. A., Jr., Toxic Trace Elements: Preferential Concentration in Respirable Particles, Science, 183, 202-204 (1974).

[4] Characterization of Metal and Polymer Surfaces, Vol. I and II, ed. by Lieng-Huang Lee, Academic Press, New York, 1977, pp. 105 and 399.

[5] Grieger, G. R. , American Laboratory, 8(4), 77 (1976).

[6] Novakov, T., Chang, S. G., and Harker, A. B., Science, 186, 259 (1974).

[7] Beliles, R. P., Metals, in Toxicology, The Basic Science of Poisons, L. J. Casarett and J. Doull, editors, Macmillan, New York (1975) pp. 454-502.

[8] Clayson, D. B. and Garner, R. C., Carcinogenic Aromatic Amines and Related Compounds, in Chemical Carcinogens, ACS Monograph 173, G. E. Searle, editor, American Chemical Society, Washington, D. C. (1976) pp. 366-461.

[9] Harrington, J. S. and,Allison, A. C., Tissue and Cellular Reactions to Particles, Fibers, and Aerosols Retained After Inhalation, in Handbook of Physiology, Sec. 9, Reactions to Environmental Agents, D. H. K. Lee, editor, American Physiol. Soc., Bethesda, MD (1977) pp. 263-283.

[10] Schroeder, H. A., Environment, 13, 18 (1971).

[11] Patterson, C. C., Arch. Environ. Health, 11, 344 (1965). 
National Bureau of Standards Special Publication 533. This Publication Originated from a Special Session on Particle Analysis, 13th Annual Conference of the Microbeam Analysis Society, Ann Arbor, MI, June 22, 1978. (Issued April 1980)

ACCURACY OF ELECTRON MICROPROBE ANALYSIS OF BIOLOGICAL FLUIDS: CHOICE OF STANDARD SOLUTIONS, AND RANGE OF LINEARITY OF THE CALIBRATION CURVES

\author{
Nicole Roine1, Lucienne Meny, \\ CEA, CEN Saclay 91190 Gif sur Yvette, France
}

and

Jean Henoc

CNET 92220 Bagneux, France

\title{
Introduction
}

In investigations of cellular mechanisms, the volume of biological fluids for analysis is often too small for conventional analytical techniques. The sensitivity of the electron nicroprobe, however, is adequate to determine elements in fluids of picoliter volumes $[8]^{1}$. The principle of the use of the electron microprobe in this case is simple. The $x$-ray intensity of an element, determined in unknown solutions, is compared with that obtained from identical volumes of standard solutions of known composition. One assumes that the emerging intensity is proportional to concentration. Absorption of $x$-rays and electrons is the main limitation to the validity of this assumption. To minimize absorption, solutions are rapidly frozen and lyophilized, which results in samples composed of submicron size crystals; in such samples, the measured x-ray intensity of an element is proportional to its concentration, even for elements of rather soft $x$-rays such as sodium [11]. The concentrations of various elements in unknown samples are directly deduced from this linear relationship, without correction for absorption, fluorescence, or atomic number effect.

This technique has now been used by many groups for the determination of concentrations of elements $[1,5,9,10,12,13]$ or organic compounds [2] in biological solutions, using either wavelength dispersive, or the energy dispersive $x$-ray detection systems $[14,15,17]$. The accuracy of this kind of analysis rests upon the assumption that the proportionality factor between background corrected signal and concentration be the same for standard and unknown solutions, at least within some range. Questions remain, however. For example: what are the limits within which the response is linear? Can any solution of known composition be used as a standard? What is the influence of the matrix upon the emerging $x$-ray intensity of the various elements? Without any theoretical formulation, these questions must be solved empirically; more particularly, each biological fluid to be analyzed necessitates a complete study of the more optimal standard solutions to insure accuracy.

The present paper attempts to answer a few of these questions and to consider the choice of the composition of the standard solutions. We compare intensities measured from real samples to those calculated for a thin film of same composition, in order to see whether the range of proportionality of the measured $x$-ray intensity to the element concentration can be deduced from a theoretical model.

${ }^{1}$ Figures in brackets indicate the literature references at the end of this paper. 
The preparation technique presently used at Saclay comprises two steps [19]: the deposit of biological and standard solutions on a specimen holder and the treatment of the dried deposits for electron microprobe analysis. This two-step procedure allows the dried samples to be stored without any special precaution and the same holder to be used repeatedly for various experiments until its surface is completely covered with samples. Subsequent electron probe analysis is automatic, directed by means of a computer program [18] which facilitates the analysis of large series of samples.

The solutions are deposited using a constriction micropipette on the polished surface of a beryllium holder. The holder surface is covered with paraffin oil to avoid spontaneous evaporation at the tip of the pipette during deposit of droplets. The oil is then washed off in a chloroform bath and the samples are allowed to dry in the open air.

Prior to electron microprobe analysis, the droplets are rehydrated by cooling the holder to $4{ }^{\circ} \mathrm{C}$ on a Peltier plate to cause water to condense from the cooled air; the atmospheric water is trapped by the salt crystals. The holder is then placed on a piece of dry ice causing the droplets to freeze immediately; they are then lyophilized at $-40{ }^{\circ} \mathrm{C}$ in a $10^{-2}$ Torr vacuum. Samples are now ready for analysis. They consist of a multitude of crystals spread over a circular area. The topography of the dried samples will be described below. The area is a function of the sample volume and of the hydrophilic property of the surface holder, which may vary from one experiment to the next. A mean sample mass thickness is calculated as the ratio of the deposited dry mass of salts to the surface area.

This technique has been applied mainly to renal physiological investigations for analysis of fluids obtained from kidney tubules by micropuncture. The principal elements are $\mathrm{Na}$ and $\mathrm{Cl}$, plus $\mathrm{K}, \mathrm{Ca}, \mathrm{P}$, and $\mathrm{Mg}$, elements whose atomic number exceeds 10 . Standard solutions are designed to have a composition as similar as possible to that of the biological samples.

The samples are fully irradiated by a defocused electron beam. Linear calibration curves are routinely obtained over the biological range of concentrations as shown in figure 1 for various elements, where x-rays are measured by crystal spectrometers. The intercept at the ordinate of the curves is the background value measured on the beryllium holder, between the samples, at the same Bragg angle. The background value is proportional to the beam intensity, and increases as a function of the atomic number of the holder and of the beam voltage. The slope of the calibration curves is also proportional to the beam intensity, the sample volume, and the inverse of the beam cross section (provided the sample is fully irradiated by the beam). The slope decreases with increasing accelerating voltage when the overvoltage ratio (ratio of accelerating voltage to the excitation potential of the analyzed line) is more than two or three. The calibration curves are linear at $12 \mathrm{kV}$ for samples of calculated mean mass thickness of $0.09 \mathrm{mg} / \mathrm{cm}^{2}$ or less. Table 1 gives typical values measured from a series of experiments of the slope-to-background ratio, which represents the ratio of the characteristic signal of $1 \mathrm{~mm} / 1$ of an element (sample volume $=0.15 \mathrm{nl}$ ) to the background signa 1 ; this ratio measured with a Cameca MS- 46 electron probe is obtained for a mass thickness of $10^{-4} \mathrm{mg} / \mathrm{cm}^{2}$ of this element. 


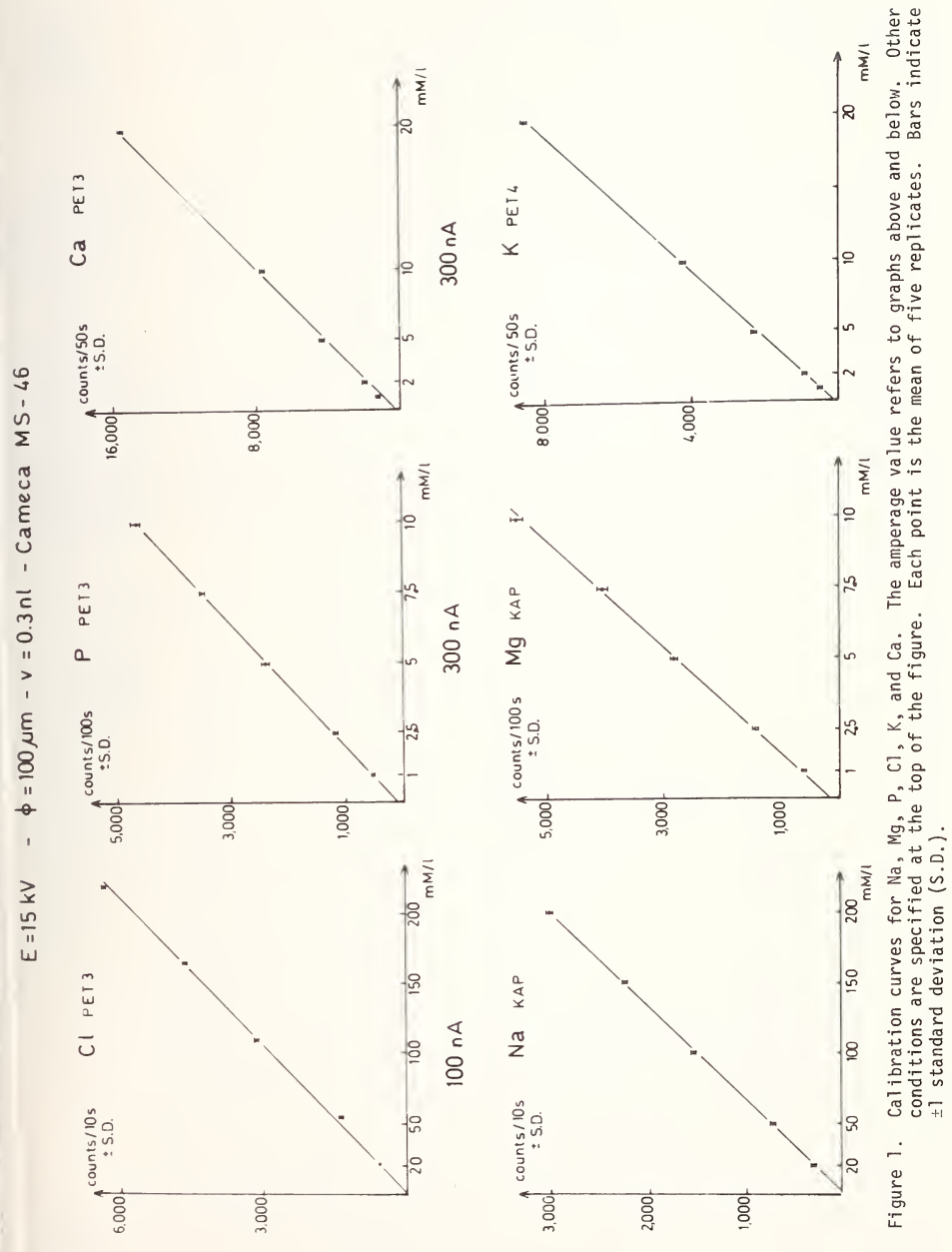


Table 1. Ratios of $1 \mathrm{mM} / 1$ concentration of an element to the background.

\begin{tabular}{|c|c|c|c|c|c|c|}
\hline \multirow{2}{*}{$\begin{array}{l}E=15 \mathrm{kV} \\
\Phi=70 \mu \mathrm{m} \\
V=0.15 \mathrm{nl}\end{array}$} & \multicolumn{2}{|c|}{$I=100 \mathrm{nA}_{j} \quad t=10 \mathrm{~s}$} & \multicolumn{2}{|c|}{$I=300 \mathrm{nA} ; t=50 \mathrm{~s}$} & \multicolumn{2}{|c|}{$I=300 n A ; t=100 \mathrm{~s}$} \\
\hline & $\begin{array}{c}\mathrm{Cl} \\
\text { PET } 3\end{array}$ & $\begin{array}{l}\mathrm{Na} \\
\mathrm{KAP}\end{array}$ & $\begin{array}{c}\text { Ca } \\
\text { PET } 3\end{array}$ & $\begin{array}{c}\text { K } \\
\text { PET } 4\end{array}$ & $\begin{array}{c}\text { P } \\
\text { PET } 3\end{array}$ & $\begin{array}{l}\mathrm{Mg} \\
\mathrm{KAP}\end{array}$ \\
\hline Background & 20 & 10 & 435 & 210 & 130 & 335 \\
\hline $1 \mathrm{mM} / \mathrm{l}$ signal & 2.0 & 1.9 & 2.6 & 2.7 & 4.1 & 2.2 \\
\hline $\begin{array}{l}\text { Background } \\
: S E ; n=13\end{array}$ & \pm 0.2 & \pm 0.1 & \pm 0.2 & \pm 0.2 & \pm 0.3 & \pm 0.2 \\
\hline $\begin{array}{l}1 \mathrm{mM} / \mathrm{l} \text { mass } \\
\text { thickness } \\
\mathrm{mg} / \mathrm{cm}^{2}\end{array}$ & $1.4 \times 10^{-4}$ & $9 \times 10^{-5}$ & $1.6 \times 10^{-4}$ & $1.5 \times 10^{-4}$ & $1.2 \times 10^{-4}$ & $9.5 \times 10^{-5}$ \\
\hline
\end{tabular}

Table 2 gives the 95 percent confidence level for precision of $1 \mathrm{~mm} / 1$ of an element measured under the conditions described in the table. The geometry of the lyophilized samples does not affect the counting rate, as shown by the fact that the standard deviation of the counting rates of five replicates of a solution is not larger than the standard deviation expected from Poisson's law [11].

Table 2. The 95 percent confidence level precision over $1 \mathrm{~mm} / 1$ measurement.

\begin{tabular}{|c|c|c|c|c|c|c|}
\hline & $\begin{array}{l}\mathrm{Na} \\
\mathrm{KAP}\end{array}$ & $\begin{array}{c}\mathrm{Cl} \\
\text { PET }\end{array}$ & $\begin{array}{l}\mathrm{Ca} \\
\mathrm{PET}\end{array}$ & $\underset{\mathrm{PET}}{\mathrm{K}}$ & $\underset{P E T}{P}$ & $\begin{array}{l}\mathrm{Mg} \\
\text { KAP }\end{array}$ \\
\hline$B G=$ background & 300 & 200 & 870 & 400 & 130 & 335 \\
\hline $\mathrm{N}=$ signal $1 \mathrm{mM} / \mathrm{l}$ & 570 & 400 & 2260 & 1080 & 533 & 737 \\
\hline$\sigma=\sqrt{N+2 B G}$ & 34.2 & 28.3 & 63.2 & 43.3 & 28.2 & 38.8 \\
\hline$\frac{t 0.05 \times 6}{\sqrt{10}}$ & 24.4 & 20.2 & 45.2 & 30.9 & 20.2 & 27.7 \\
\hline Precision \% & 4.3 & 5.0 & 2.0 & 2.9 & 3.8 & 3.8 \\
\hline $3 \sqrt{B G}$ & 52.0 & 42.2 & 88.5 & 60.0 & 34.2 & 55.0 \\
\hline $\begin{array}{l}\text { minimum detecta } \\
\text { ble concentration } \\
\mathrm{mM} /\end{array}$ & 0.09 & 0.10 & 0.04 & 0.05 & 0.06 & 0.07 \\
\hline $\begin{array}{l}\text { mass thickness } \\
\mathrm{mg} / \mathrm{cm}^{2}\end{array}$ & $8 \times 10-6$ & $1.4 \times 10^{-5}$ & $6 \times 10^{-6}$ & $7 \times 10-6$ & $7 \times 10^{-6}$ & $7 \times 10^{-6}$ \\
\hline
\end{tabular}

probe: $15 \mathrm{kV}-70 \mu \mathrm{m}-100$ seconds - sample volume : $0.15 \mathrm{nl}$

$\mathrm{Cl}: 100 \mathrm{nA}$ - others : $300 \mathrm{nA}$

Table 2 gives, under the same analysis conditions, the minimum detectable concentration as defined by a signal equal to three times the standard deviation of the background. The minimum detectable concentration corresponds to an element mass thickness of $10^{-16}$ $\mathrm{g} / \mu \mathrm{m}^{2}$, or $70^{-5} \mathrm{mg} / \mathrm{cm}^{2}$. It can be improved by increasing the slope-to-background ratio, or 
ncreasing the number of counts. The slope-to-background ratio is increased by lowering che beam voltage, provided this does not affect the linearity of the calibration curves (by absorption of the electrons within the samples). The number of counts is increased by ncreasing the analysis time or the beam intensity, provided this does not affect the tability of the counting rate.

\section{Accuracy of Results}

The validity of using calibration curves without correction for absorption, fluorescence, and atomic number depends upon: 1) the identity of the oxidation degree when it affects the energy of the line to be analyzed; 2) the stability of the counting rate of the various elements; and 3 ) the similarity of standard and unknown solutions in terms of the nain matrix. Only the first and second points will be considered here.

\section{Influence of the degree of oxidation}

Among the various elements of interest for the biologist, sulfur exhibits different degrees of oxidation, either as sulfate in the tubular fluid of the kidney, or with an ; $-\mathrm{H}$ bond in the amino acids cysteine, cystine and methionine. According to Faessler and joehring [4], an increase of the $K \alpha$ energy of $1.2 \mathrm{eV}$ from $\mathrm{S}^{\circ}$ to $\mathrm{S}^{6^{+}}$results in a decrease of $0.003 \AA$ of the wavelength of sulfur. This decrease is easily observed with the PET crystal of a CAMECA MS-46 microprobe. Figure 2 illustrates the $S$ signal, measured from julfate, cysteine, and methionine samples, as a function of the PET crystal position. The

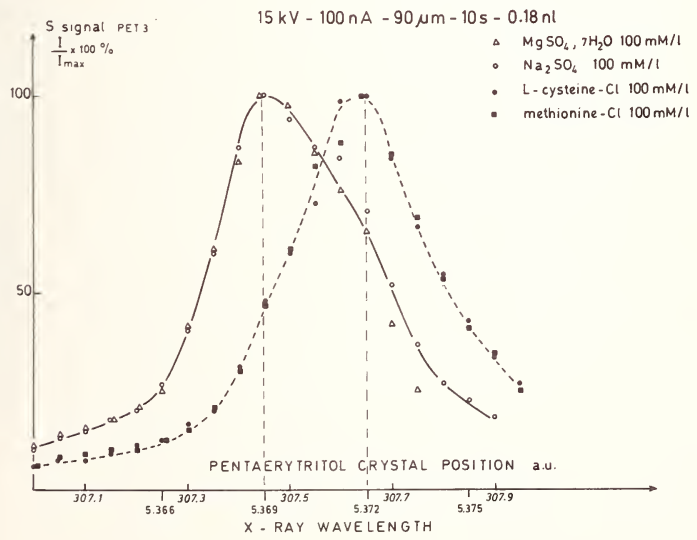

Figure 2. The characteristic signal of sulfur from various sulfur containing solutions as a function of the crystal spectrometer position (higher figures) and of the corresponding wavelength value (lower figures). a.u. = arbitrary units. 


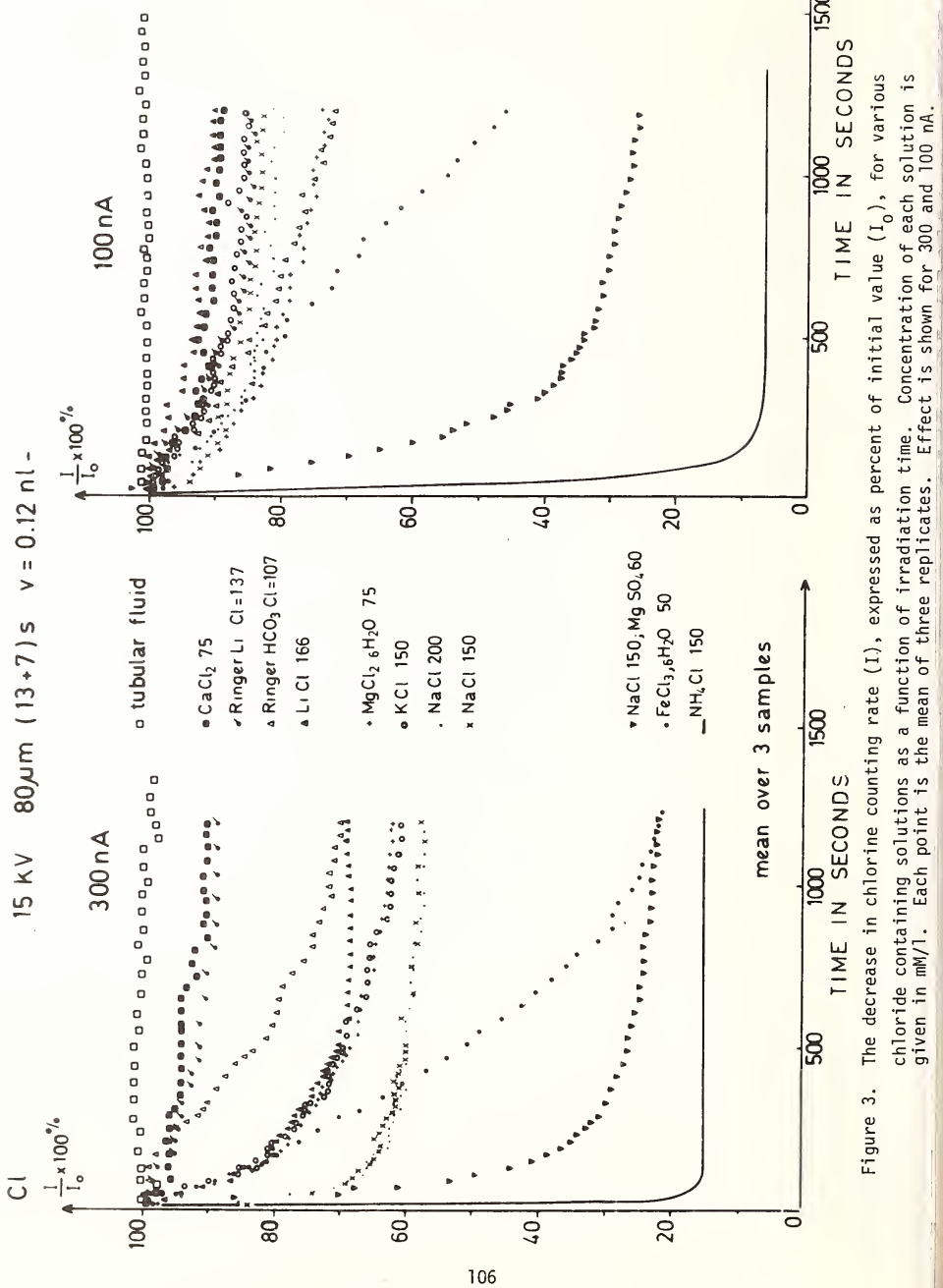


ignal is expressed as a percentage of the highest counting rate. Samples are measured at beam current density which does not affect the stability of the sulfur signal counting ate. The sulfate curve is clearly different from the methionine and cysteine curves. nly 50 percent of the maximal cysteine and methionine signals are measured when the crystal pectrometer is positioned for the maximal sulfate signal [20]. The measurement of sulfur n samples containing various sulfur compounds must take this into account.

\section{Stability of the counting rates}

The counting rates of $\mathrm{Na}, \mathrm{Mg}, \mathrm{P}, \mathrm{Ca}$, and $\mathrm{K}$ are stable in both biological and mineral olutions (when they are mixtures of salts) for at least $1000 \mathrm{~s}$ irradiation with a beam urrent density as high as $0.008 \mathrm{~A} / \mathrm{cm}^{2}$. The chlorine counting rate is stable in biological luids such as plasma, urine, and kidney tubular fluid. It decreases with time, however, n mineral solutions when the probe current density and the sample mass thickness increase 19]. This effect is largely dependent on the nature of the accompanying cation (figure i). For example, ammonium chloride has a particularly volatile chlorine signal. On the ame figure is shown the influence of the beam intensity on the time-dependent decrease in hlorine counting rate.

Figure 4 shows that when a pure mineral chloride salt is irradiated, the cation ounting rate decreases, along with chlorine, but to a lesser extent. Moreover, as shown $n$ the same figure, samples obtained from identical volumes of the same solution do not ose their chlorine and cation signals at the same rate, probably due to variations in the eplicate mass thickness.

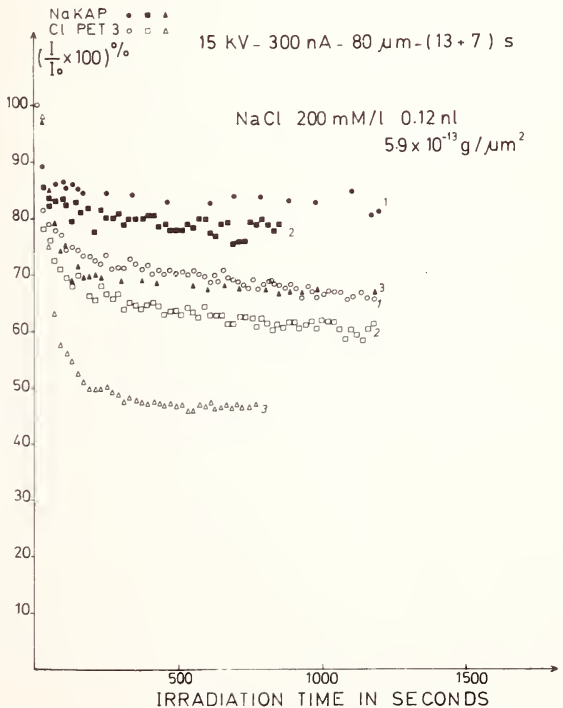

Figure 4. The decrease in chlorine and sodium counting rate as a function of irradiation time. Number at the end of each line identifies replicates. 
Bromine and iodine behave like chlorine; their counting rates decrease with the time in mineral solutions (figures 5 and 6 ). In a solution of a pure salt, the counting rate of the accompanying cation decreases too, but always to a lesser extent.

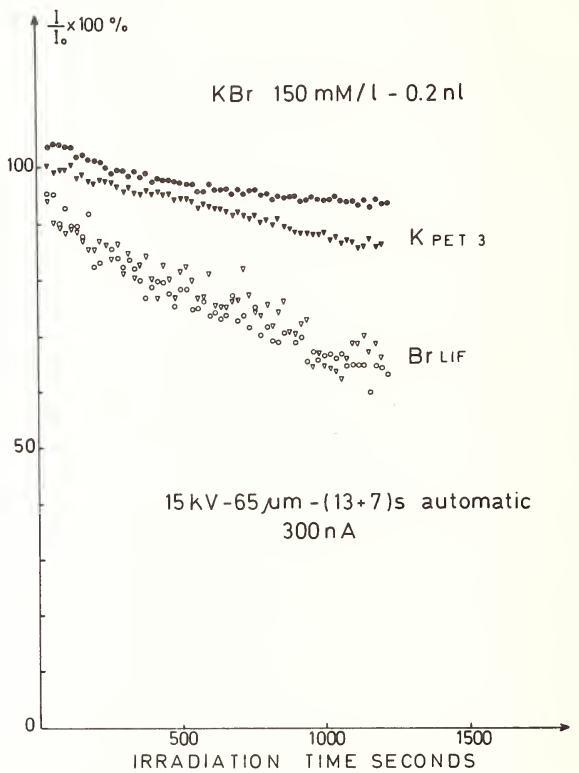

Figure 5. The decrease in bromine and petassium counting rate as a function of irradiation time. Open symbol - bromine signal. Black symbol - potassium signal. The same symbols refer to the same replicates. 


$$
\frac{1}{1} \times 100 \%
$$

\section{$\mathrm{KI} 150 \mathrm{mM} / \mathrm{l} 0.2 \mathrm{nl}$}

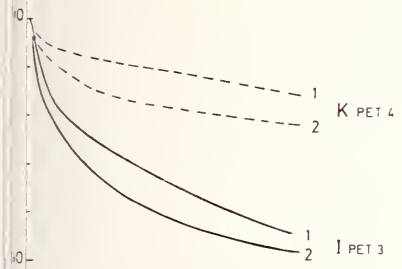

$15 \mathrm{kV}-65 \mu \mathrm{m} \cdot(13+7) \mathrm{s}$
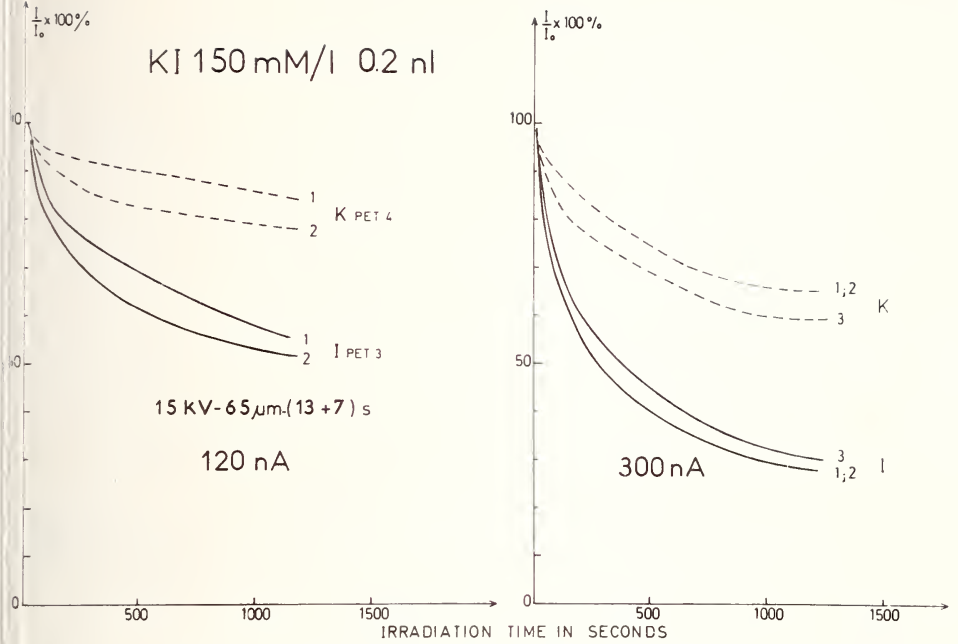

Figure 6. The decrease in potassium and iodine counting rate as a function of irradiation time. Effect is shown for 120 and $300 \mathrm{nA}$. Number at the end of the lines identify replicates.

Instability with irradiation time of the sulfur signal is also observed in sulfate solutions, but not in methionine or cysteine solutions (figure 7). A non-hydrated sulfate salt always exhibits a decrease of its $S$ signal, and to a lesser extent of its cation signal, with time (figure 8 ). Hydrated sulfate salts, such as $\mathrm{MgSO}_{4}, 7 \mathrm{H}_{2} \mathrm{O}$, exhibit either a decrease (figure 9) or an increase (figure 10) of their S signal, with a corresponding change of the cation signal (figure 10). 


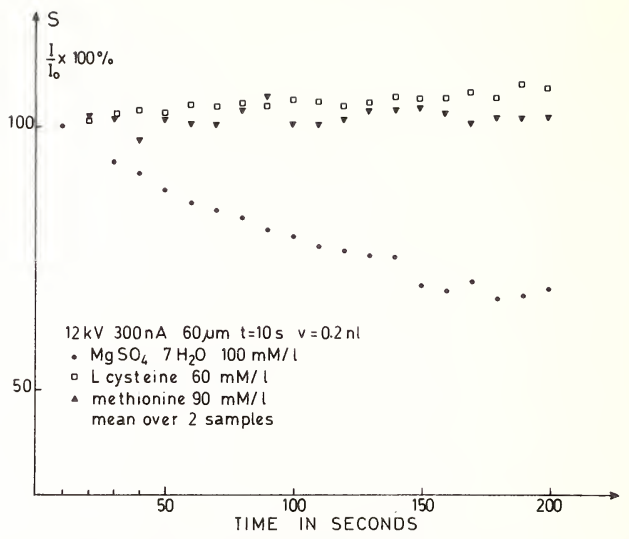

Figure 7. The variation in the sulfur counting rates as a function of irradiation time for various sulfur containing solutions.

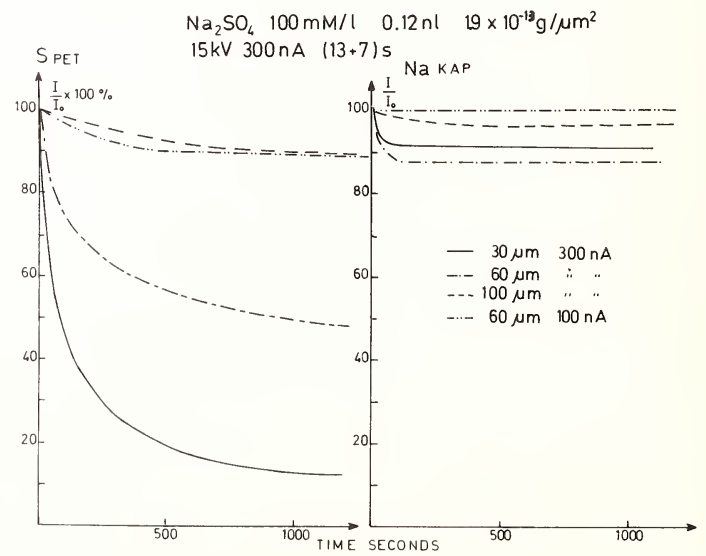

Figure 8. The decrease in sulfur and sodium counting rate as a function of irradiation time in a solution of sodium sulfate. Lines refer to various probe current densities. 
$\mathrm{Mg} \mathrm{SO}_{4,7} \mathrm{H}_{2} \mathrm{O} \quad 10 \mathrm{~mm} / \mathrm{l}$

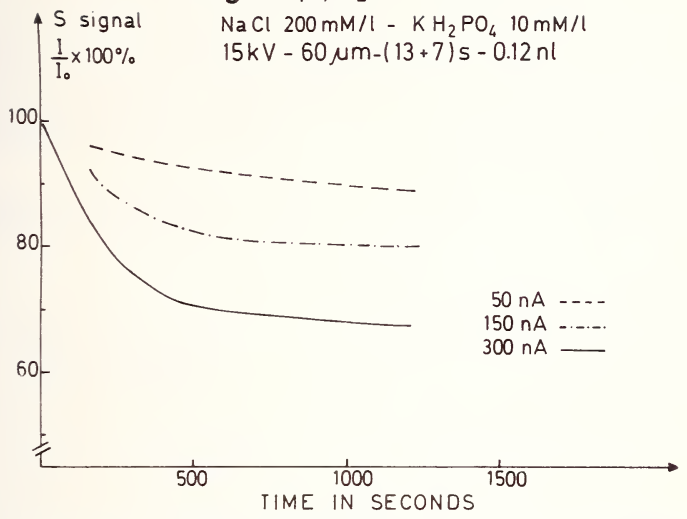

Figure 9. The decrease in sulfur counting rate as a function of irradiation time from a hydrated sulfate solution mixed with other salts. Lines refer to various probe current densities.

$\mathrm{Mg} \mathrm{SO}_{4} \mathrm{H}_{2} \mathrm{O} \quad 100 \mathrm{~mm} / / \quad 0.12 \mathrm{nl}$

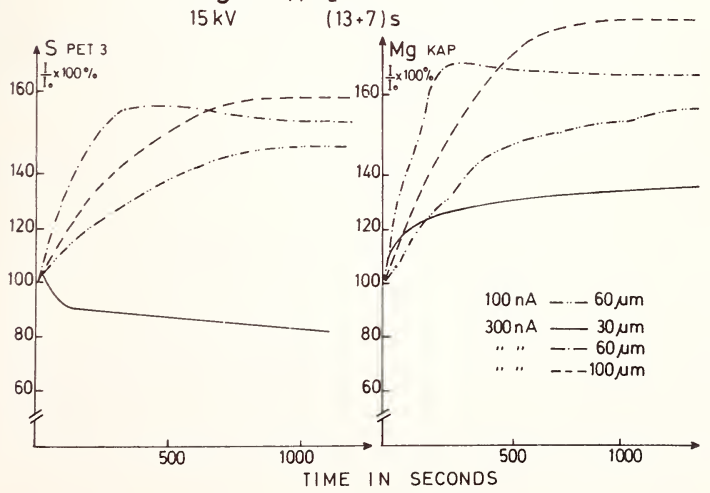

Figure 10. The change in sulfur and magnesium counting rate as a function of irradiation time from a hydrated sulfate solution. Lines refer to various probe current densities. 
Because of this variability, comparison of signals of biological solutions, which are stable with time, to calibration curves obtained from mineral solutions, which are not, would lead to errors in the determination of the concentrations of certain elements in biological fluids. To obtain an accurate analysis, it is essential to have stable counting rates. We have achieved stability either by: 1) using lower beam current densities $\left(2 \times 10^{-3} \mathrm{~A} / \mathrm{cm}^{2}\right.$ is correct for chlorine measurement within samples with a mass thickness of $0.06 \mathrm{mg} / \mathrm{cm}^{2}$ ); 2) cooling the samples (a cooling to only $-20^{\circ} \mathrm{C}$ is sufficient); or 3 ) adding organic material to the mineral solutions (e.g. urea). Problems of instability are specific to the wavelength dispersive system, which requires higher beam densities than the energy dispersive system; neither Rick et a1. [17] nor Quinton $[14,15]$ observed instability of the signal with the latter system.

Stability of the counting rates of the various elements is obtained with the mixtures of salts shown in figure 11 which are used as standard solutions in our laboratory.

$\mathrm{NaCl} 200 \mathrm{mM} /-\mathrm{MgCl}_{2},{ }_{6} \mathrm{H}_{2} \mathrm{O} 10 \mathrm{mM} / \mathrm{l}$ $\mathrm{KH}_{2} \mathrm{PO}_{4} 10 \mathrm{mM} / \mathrm{l}$
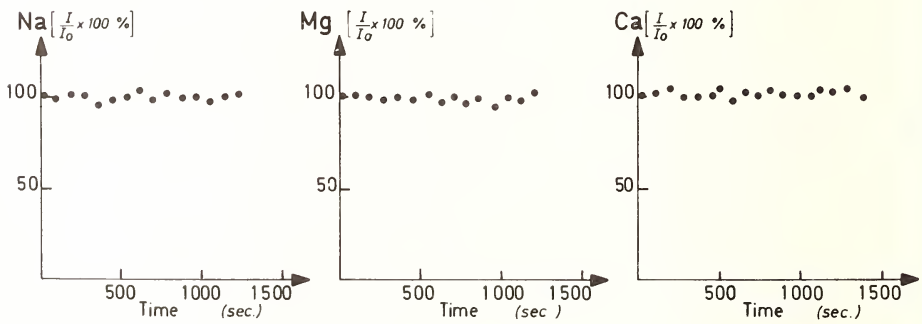

\section{$\mathrm{CaCl}_{2}=\mathrm{KCl}=20 \mathrm{mM} / \mathrm{l}$}

$\mathrm{NaCl}=100 \mathrm{mM} / \mathrm{l}$

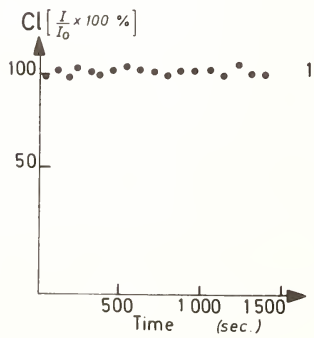

$v=0.2 \mathrm{ml} .15 \mathrm{kV} .80 \mu \mathrm{m}$.

Cl: $100 \cap \mathrm{A}$ - others: $300 \cap \mathrm{A}$
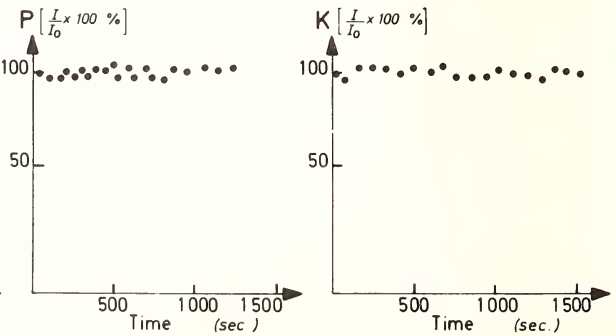

Figure 11. Stability of the counting rates of the elements from solutions used as "standard solutions". 
Chlorine is the first element measured, with a beam density of $0.002 \mathrm{~A} / \mathrm{cm}^{2}$. The accuracy of the measurement of the chlorine signal within tubular fluid extracted from kidney structures is shown by figure 12 which compares the results of the quantitative analysis of chlorine performed both by electron microprobe and by the Ramsay microcoulometric method [16]. The equation of the regression line is: $y=0.94 x+52, n=72, r=0.94$. The probability for the slope to be different from unity is smaller than 10 percent. The range of chlorine concentration is larger than that of the calibration curves; samples were therefore diluted for microprobe analysis, to fit within the range of concentration of the calibration curves.

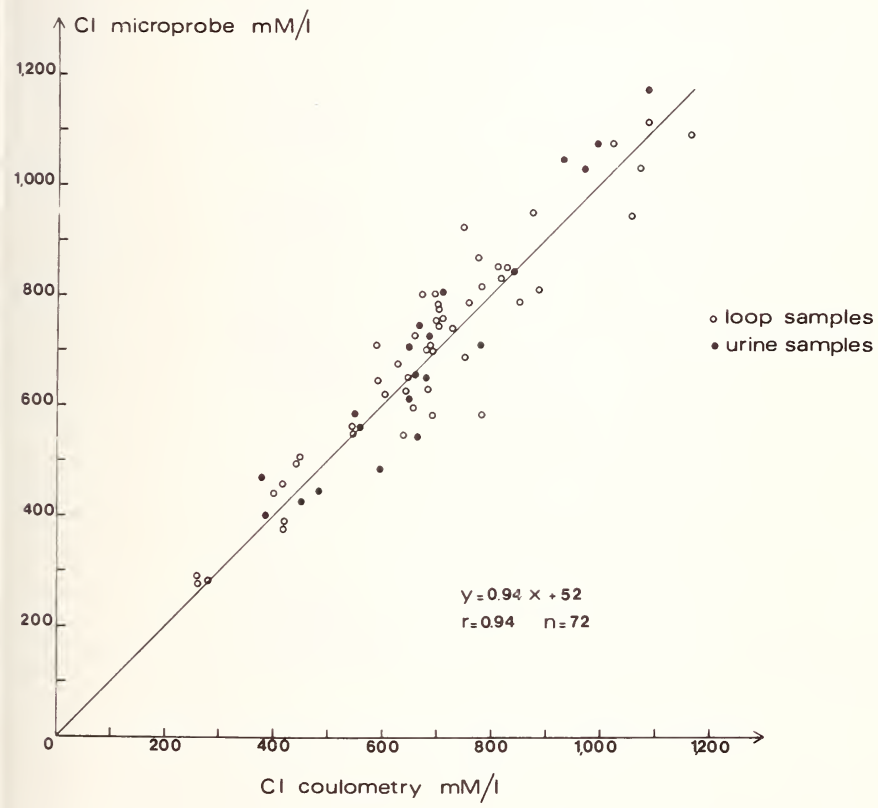

Figure 12. Comparison of chlorine measurements obtained by electron microprobe analysis and Ramsay coulometric method. Line indicates identity. "Loop samples" refer to tubular fluid samples from loops of Henle.

The Range of Linearity for the Calibration Curves

Standard solutions for measurement of $\mathrm{Na}, \mathrm{Cl}, \mathrm{P}$, and $\mathrm{Mg}$ signals ( $\mathrm{H}$ series) consist of a mixture of $\mathrm{NaCl}, \mathrm{KH}_{2} \mathrm{PO}_{4}$ and $\mathrm{MgCl}_{2}, 6 \mathrm{H}_{2} \mathrm{O}$ salts in constant percentage, diluted to vary the concentrations by a factor of 10 . Standard solutions for measurement of $\mathrm{Ca}$ and K (G series) consist of solutions of $100 \mathrm{mM} / 1 \mathrm{NaCl}$, plus concentrations of $\mathrm{CaCl}_{2}$ and $\mathrm{KCl}$ varying by a 
factor of 20. Details are given in Table 3. Aside from the necessary precautions to insure stability of the counting rates of the elements, practical considerations guided the choice of the composition of the standard solutions: 1) calcium and phosphorus tend to precipitate together when their concentrations increase, so that each is present in only one series; 2) only two elements of atomic number less than 20 can be measured simultaneously with our microprobe; 3 ) these two elements are chosen according to the ranges of crystal spectrometers and the required analysis time.

Table 3. Composition of the standard solutions.

\begin{tabular}{|c|c|c|c|c|c|c|c|c|}
\hline H solutions & $\begin{array}{l}\mathrm{NaCl} \\
\mathrm{mM} / 1\end{array}$ & $\begin{array}{c}\mathrm{KH}_{2} \mathrm{PO}_{4} \\
\mathrm{mM} / 1\end{array}$ & $\begin{array}{l}\mathrm{MgCl}_{2} \\
6 \mathrm{H}_{2} \mathrm{O} \\
\mathrm{mM} / 1\end{array}$ & $\begin{array}{l}\text { Total } \\
\text { dry mass } \\
\mathrm{g} / 1\end{array}$ & $\begin{array}{r}\mathrm{Na} \\
\%\end{array}$ & $\begin{array}{l}\mathrm{Cl} \\
\%\end{array}$ & $\begin{array}{l}\mathrm{Mg} \\
\%\end{array}$ & $\begin{array}{l}k \\
\%\end{array}$ \\
\hline 1 & 20 & 1 & 1 & 1.48 & 31.1 & 52.8 & 1.6 & 2.7 \\
\hline 2 & 50 & 2.5 & 2.5 & 3.69 & " & $"$ & $"$ & " \\
\hline 3 & 100 & 5 & 5 & 7. 38 & $"$ & " & $"$ & " \\
\hline 4 & 150 & 7.5 & 7.5 & 11.07 & " & " & " & " \\
\hline 5 & 200 & 10 & 10 & 14.76 & $"$ & $"$ & $" 1$ & $"$ \\
\hline \multirow{2}{*}{ G solutions } & $\mathrm{NaCl}$ & $\mathrm{KCl}$ & $\mathrm{CaCl}_{2}$ & \multirow{2}{*}{$\begin{array}{l}\text { Total } \\
\text { dry mass } \\
\mathrm{g} / 1\end{array}$} & $\mathrm{Na}$ & $\mathrm{Cl}$ & k & $\mathrm{Ca}$ \\
\hline & $\mathrm{mM} / 1$ & $\mathrm{mM} / 1$ & $\mathrm{mM} / 1$ & & $\%$ & $\%$ & $\%$ & $\%$ \\
\hline 1 & 100 & 1 & 1 & 6.03 & 38.1 & 60.6 & 0.65 & 0.66 \\
\hline 2 & 100 & 2 & 2 & 6.22 & 37.0 & 60.45 & 1.26 & 1.29 \\
\hline 3 & 100 & 5 & 5 & 6.77 & 34.0 & 60.1 & 2.9 & 3.0 \\
\hline 4 & 100 & 10 & 10 & 7.70 & 29.9 & 59.8 & 5.1 & 5.2 \\
\hline 5 & 100 & 20 & 20 & 9.55 & 24.1 & 59.3 & 8.2 & 8.4 \\
\hline
\end{tabular}

\section{Scanning electron microscope images of standard solutions}

After lyophilization, the standard solutions and the biological fluids appear as innumerable submicron size crystals distributed over a circular surface. Photographs were taken of the samples with a Cambridge Stereoscan scanning electron microscope operated at $10 \mathrm{kV}$, with a sample current measured on the support of $5 \times 10^{12} \mathrm{~A}$. The resolution is 25 $\mathrm{nm}$. The holder was tilted at 30 degrees.

$\mathrm{H}$ series. Figure 13 shows the final appearance of samples with a volume of $0.08 \mathrm{nl}$. It is to be noted that the sample surface increases with dry mass for the first two solutions and then remains constant for the last three solutions. The mean value of the sample mass thickness is clearly only a rough estimate of the mass thickness, as samples are composed of piles of discrete crystals. Figure 14 shows a picture of those samples at higher magnification. The size of the discrete crystals ranges from $25 \mathrm{~nm}$ to $150 \mathrm{~nm}$, independently of mean mass thickness. Thus, the increase in mass thickness is manifested by a higher number of particies per unit of surface area. Furthermore, the number of crystals per unit of surface is smaller at the extreme periphery than in the center. Figure 15 shows pictures taken of $0.3 \mathrm{nl}$ volumes of the same solutions. When the volume increases, the sample area increases, so that the increase in mean mass thickness is not proportional to the increase in volume. At higher magnification (figure 16) the range of crystal size is seen to be the same as that of samples of $0.08 \mathrm{nl}$ volumes. For the $0.3 \mathrm{nl}$ samples, the increase in the mass thickness is due to a higher number of crystals per unit of surface area. 

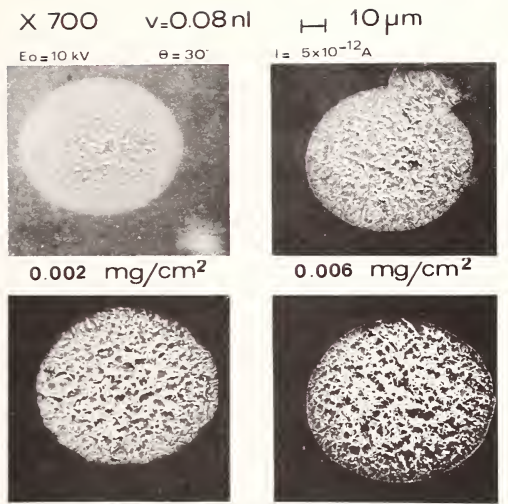

$$
0.010 \mathrm{mg} / \mathrm{cm}^{2}
$$

$$
0.015 \mathrm{mg} / \mathrm{cm}^{2}
$$

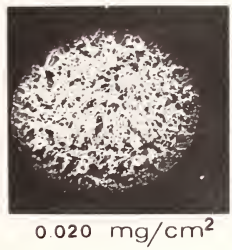

$$
\begin{aligned}
& \mathrm{Na}=31.1 \% \\
& \mathrm{Cl}=52.8 \% \\
& P=2.1 \% \\
& \mathrm{Mg}=1.6 \% \\
& \mathrm{~K}=2.7 \% \\
& \mathrm{H} \text { and } \mathrm{O}=9.8 \%
\end{aligned}
$$

Figure 13. SEM (scanning electron microscope) images of $0.08 \mathrm{nl}$ of $\mathrm{H}$ solutions; magnification X700. 


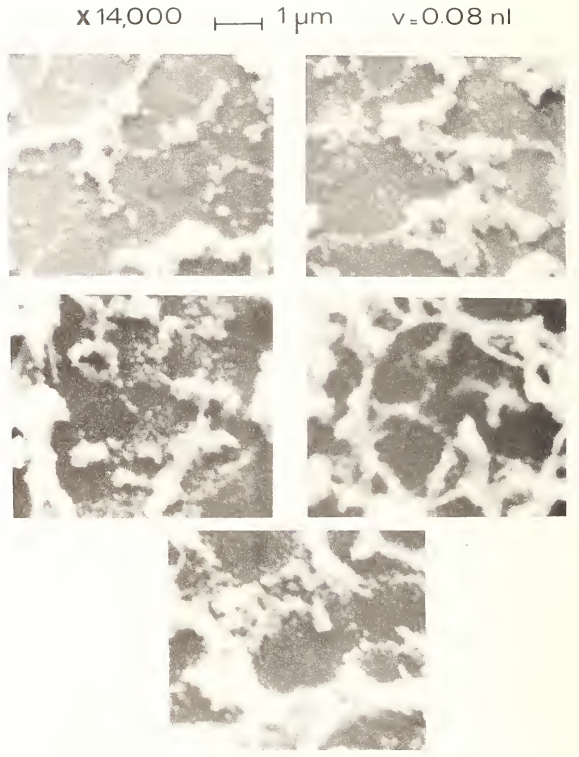

Figure 14. SEM images of $0.08 \mathrm{nl}$ of $\mathrm{H}$ solutions; magnification $\mathrm{X} 14,000$. 
$x=700 \quad v=0.3 \mathrm{nl}$

$E_{0}=10 \mathrm{kV}$

$\theta=30^{\circ}$
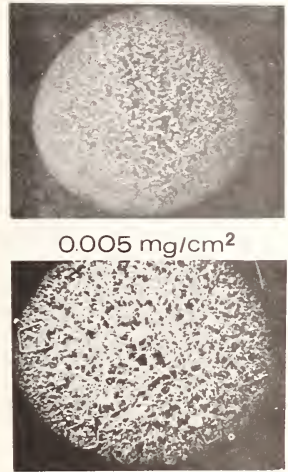

$0.020 \mathrm{mg} / \mathrm{cm}^{2}$

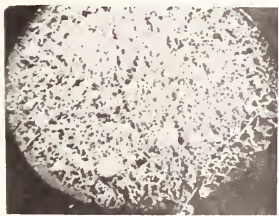

$0034 \mathrm{mg} / \mathrm{cm}^{2}$ $\longmapsto 10 \mu \mathrm{m}$

$1=5 \times 10^{-12} \mathrm{~A}$

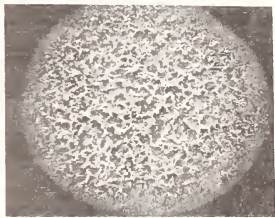

$0.010 \mathrm{mg} / \mathrm{cm}^{2}$

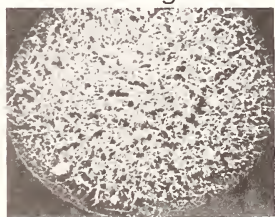

$0.026 \mathrm{mg} / \mathrm{cm}^{2}$

$\mathrm{Na}=31.1 \%$

$\mathrm{Cl}=52.8 \%$

$P=2.1 \%$

$\mathrm{Mg}=1.6 \%$

$K=2.7 \%$

$\mathrm{H}$ and $\mathrm{O}=9.8 \%$

Figure 15. SEM images of $0.3 \mathrm{nl}$ of $\mathrm{H}$ solutions; magnification $X 700$. 


\section{$0.005 \mathrm{mg} / \mathrm{cm}^{2}$}
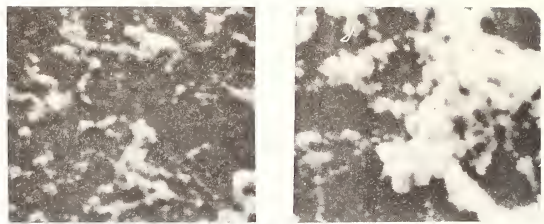

$0.035 \mathrm{mg} / \mathrm{cm}^{2}$
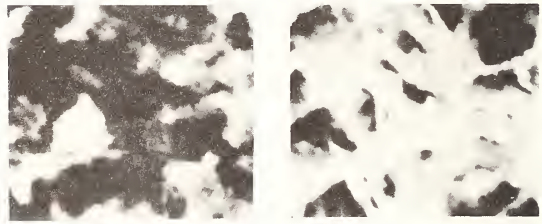

$v=0.3 n !$

$\mathbf{X} 14,000$

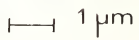

Figure 16. SEM images of $0.3 \mathrm{nl}$ of $\mathrm{H}$ solutions; magnification $\times 14,000$. Left: periphery of the samples; right: center of the samples.

G series. The five standard solutions ( $G$ series) differ in appearance from those of $H$ series and from each other (figure 17). The surface is smaller at equivalent volumes than for $\mathrm{H}$ solutions. This is due to the lack of phosphate ion - this ion reduces surface tension of a solution. The picture at higher magnification (figure 18) shows that, in contrast to what occurs in samples of $\mathrm{H}$ series of constant composition, the size of the crystals varies as the concentrations of $\mathrm{Ca}$ and $\mathrm{K}$ increase; the size of crystals increases from a range of 25-150 nm (lowest standard) to a range of 200-500 nm (highest standard). Figures 19 and 20 represent samples $0.3 \mathrm{nl}$ in volume. The range of crystal size is the same as that with the smaller pipette volume for each solution. The increase of mass thickness is associated with a corresponding increase in the number of particles per unit of surface area. Again the number of crystals per unit of surface is smaller at the extreme periphery than in the center. 


$$
\begin{array}{lrr}
X 700 & \mapsto 10 \mu \mathrm{m} \\
E_{0}=10 \mathrm{kV} & \mathrm{I}=5 \times 10^{-12} \mathrm{~A} & \theta=30^{\circ}
\end{array}
$$

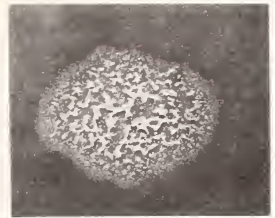

$0.012 \mathrm{mg} / \mathrm{cm}^{2}$
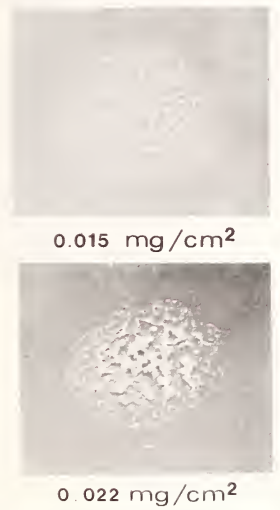

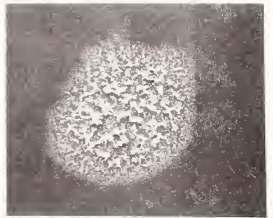

$$
0.014 \mathrm{mg} / \mathrm{cm}^{2}
$$

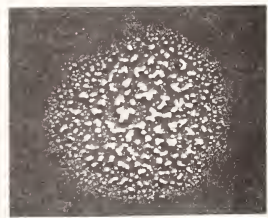

$0.015 \mathrm{mg} / \mathrm{cm}^{2}$

$\mathrm{NaCl} 100 \mathrm{~mm} / \mathrm{l}$

$\mathrm{CaCl}_{2}, 1,2,5,10$, $\mathrm{KCl} 20 \mathrm{mM} / \mathrm{I}$

Figure 17. SEM images of $0.08 \mathrm{nl}$ of $E$ solutions; magnification $X 700$. 


\section{$\times 14,000 \longmapsto r^{1} \mu \mathrm{m} \quad v=0.08 \mathrm{nl}$}
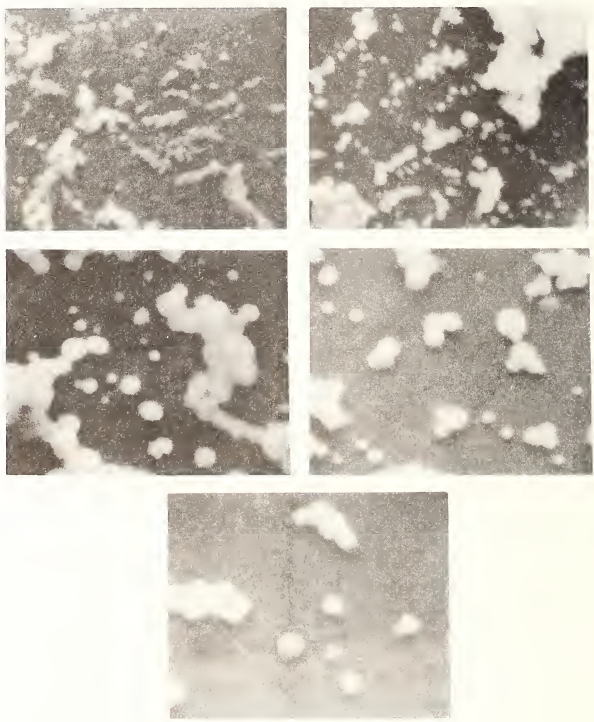

Figure 18. SEM images of $0.08 \mathrm{n} 1$ of $G$ solutions; magnification $X 14,000$. 


\section{$X 700 \quad \mapsto 10 \mu \mathrm{m} \quad v=0.3 \mathrm{nl}$ \\ $E_{\circ}=10 \mathrm{kV}$ \\ $\mathrm{I}=5 \times 10^{-12} \mathrm{~A} \quad \theta=30^{\circ}$}
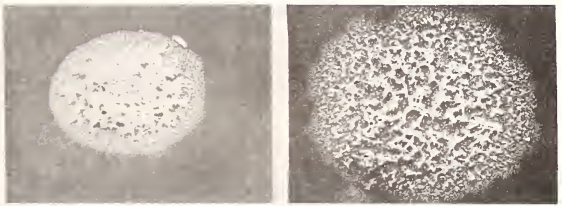

$0.046 \mathrm{mg} / \mathrm{cm}^{2}$

$0.024 \mathrm{mg} / \mathrm{cm}^{2}$
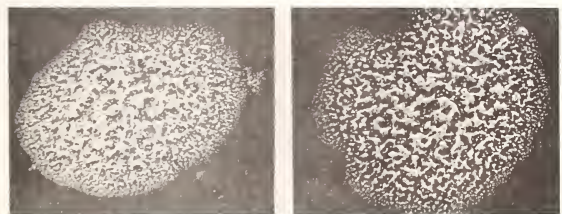

$0.026 \mathrm{mg} / \mathrm{cm}^{2}$

$0.030 \mathrm{mg} / \mathrm{cm}^{2}$

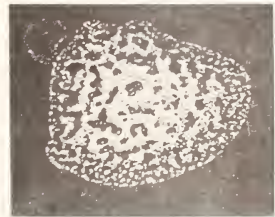

$\mathrm{NaCl} 100 \mathrm{mM} / 1$

$\mathrm{CaCl}_{2}, 1,2,5,10$. $\mathrm{KCl} 20 \mathrm{mM} / \mathrm{I}$

$0.047 \mathrm{mg} / \mathrm{cm}^{2}$

Figure 19. SEM images of $0.3 \mathrm{n} 1$ of $G$ solutions; magnification $\times 700$. 


\section{$\times 14,000 \longmapsto 1 \mu m \quad v=0.3 \mathrm{nl}$}
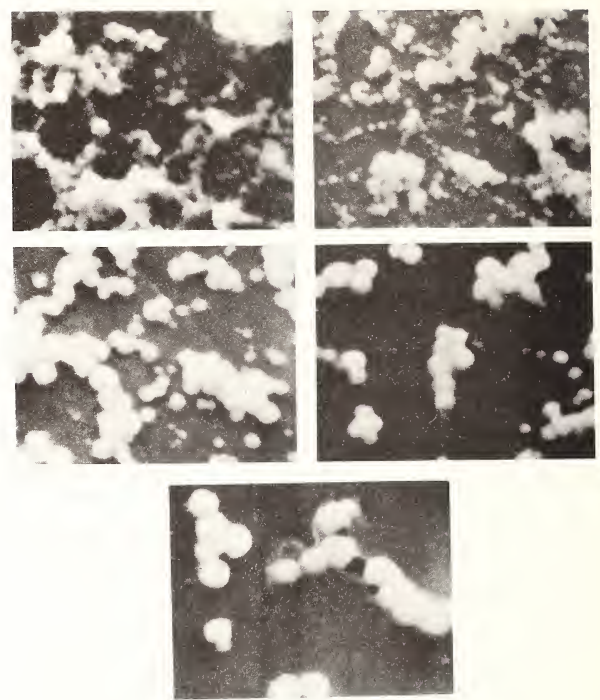

Figure 20. SEM images of $0.3 \mathrm{nl}$ of $\mathrm{G}$ solutions; magnification $\mathrm{X} 14,000$.

These observations show that the size of the crystals seems to depend upon the composition of the solution independently of its dry mass. The increase in dry mass is associated with an increase in the number of crystals per unit of surface area; moreover the number of crystals per unit of surface area is larger in the center than at the periphery of the samples.

\section{Experimental range of linearity of calibration curves}

The range of linearity of calibration curves is shortened by electron and/or $x$-ray absorption [11]. Electron absorption, which causes saturation curves for the thickest samples, is suppressed by increasing accelerating voltage. X-ray absorption depends on the energy of the line and on the matrix; the loss of linearity of the calibration curves cannot be corrected by an increase of the accelerating voltage.

Experimentally, the calibration curves are linear at $10 \mathrm{kV}$ for a mass thickness up to $0.07 \mathrm{mg} / \mathrm{cm}^{2}$, and at $12 \mathrm{kV}$ for a mass thickness up to $0.09 \mathrm{mg} / \mathrm{cm}^{2}$. (For a given solution, the final mass thickness can be varied by changing the volume of the sample and the hydrophilic property of the holder surface - the latter is accomplished by using chloroform to remove grease). It is important to note that calibration curves are linear for solutions of $400 \mathrm{milliosmols} / \mathrm{kg}$ water or less, i.e. approximately iso-osmotic or hypo-osmotic to mammalian plasma. Some fluids, notably those of the renal medulla, are hyper-osmotic. It is necessary to reduce their osmolality by diluting them with water. 
To investigate the extent of linearity of calibration curves, solutions with $\mathrm{HI}-\mathrm{H} 5$ and composition and increasing dry mass are used. The surface area of those samples is arly constant, so that their mass thickness increases with the dry mass. The samples are radiated with discrete high voltage values (12 kV - $15 \mathrm{kV}-20 \mathrm{kV}-25 \mathrm{kV}$ ) in a CAMECA -46 microprobe with a $18^{\circ}$ take-off angle.

For each element, the measured intensity is converted to "apparent concentration" by near extrapolation of the calibration curves of figure 1 to the higher concentrations dicated in figures 21 and 22. This "apparent concentration" is reported as a function of e real concentration for the different high voltage values and for any element on the gures 21 and 22 . The deviation from linearity (represented by the continuous 1 ine) is st marked for the low voltage values, where a saturation effect is obtained for each ement. When the voltage increases, the measured intensities are proportional to concenation within a larger range, especially for elements with higher atomic numbers.

$$
\begin{aligned}
& -25 \mathrm{kV}=20 \mathrm{kV} \quad+15 \mathrm{kV} \quad 012 \mathrm{kV} \quad 0.3 \mathrm{nl} \quad 110 \mu \mathrm{m} \\
& \mathrm{Na}=31.1 \% \quad \mathrm{Cl}=52.8 \% \quad \mathrm{P}=2.1 \% \quad \mathrm{Mg}=1.6 \% \quad \mathrm{~K}=2.6 \% \quad \mathrm{H} \text { and } \mathrm{O}=9.8 \%
\end{aligned}
$$
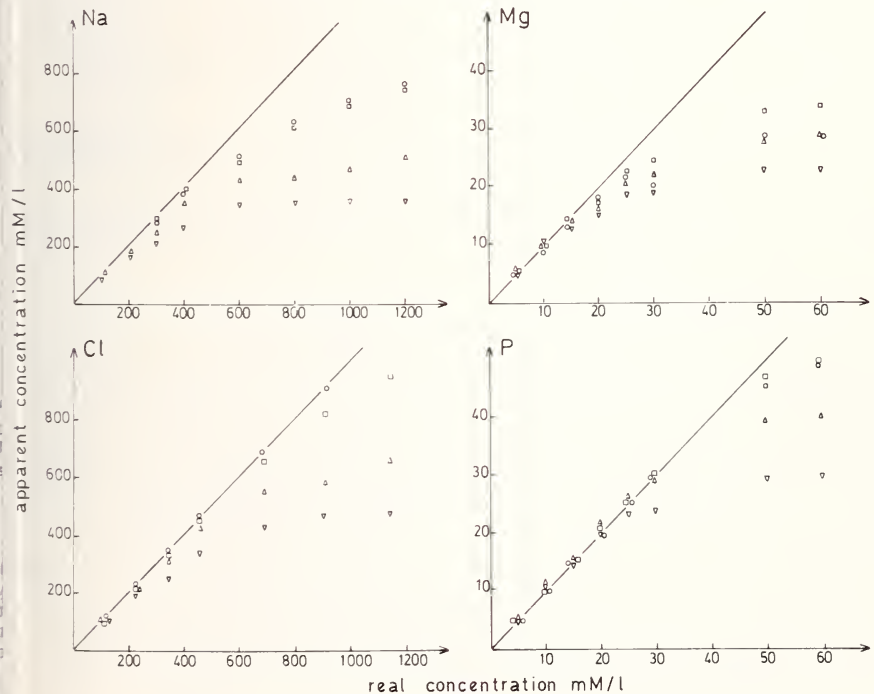

gure 21. The apparent concentration, measured by extrapolation of calibration curves like those of figure 1 , is shown as a function of the actual for $H$ solutions of increasing dry mass. 

$I=300 \mathrm{nA}$
$\phi=110 \mu \mathrm{m} \quad v=0.3 \mathrm{nl}$
$025 \mathrm{kV}$
$020 \mathrm{kV}$
$\mathrm{Na}=34 \%$$$
\mathrm{Cl}=60.1 \%
$$
$\Delta 15 \mathrm{kV}$
$\nabla 12 \mathrm{kV}$
$110 \mu \mathrm{m}$

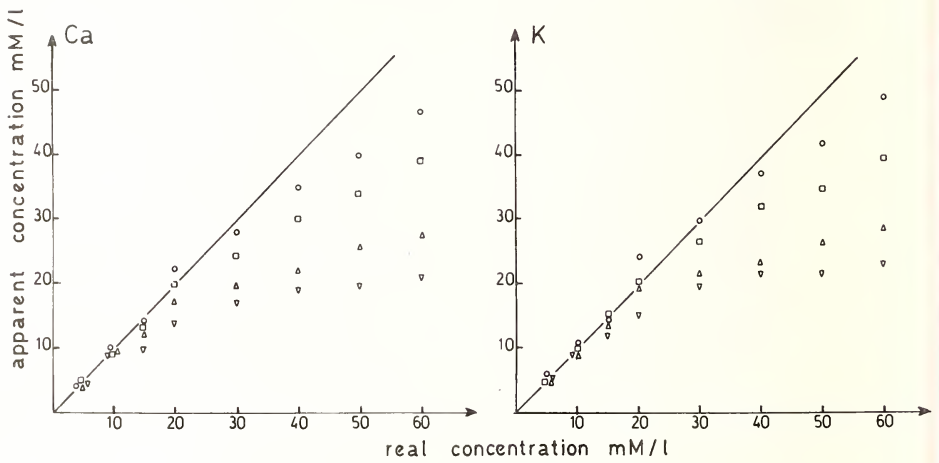

Figure 22. The apparent concentration, measured by extrapolation of calibration curves like those of figure 1 , is shown as a function of the actual for G3 solutions of increasing dry mass.

Curves as depicted in figures 21 and 22 cannot be used to correct for deviation fron linearity, since they vary from one preparation to another, depending on the surface arei of the samples. The ratio of the apparent to actual concentration has been investigatec for each element at different accelerating voltages as a function of the calculated sample mass thickness. For each element a curve is then obtained, which indicates the lack of linearity of the extrapolated calibration curves as a function of the sample mass thickness and of voltage (figures 23 and 24 ). At $12 \mathrm{kV}$ the deviation from linearity of the extrapolatec calibration curves is the same for all the elements. At $15 \mathrm{kV}$ the deviation from linearits. starts below $0.15 \mathrm{mg} / \mathrm{cm}^{2}$ for $\mathrm{Na}$ and $\mathrm{Mg}$, and at higher mass thickness for other elements. At $25 \mathrm{kV}$, the measured intensities of $\mathrm{Cl}, \mathrm{K}, \mathrm{Ca}$, and $\mathrm{P}$ are still proportional to the concentration for an estimated mass thickness of at least $0.3 \mathrm{mg} / \mathrm{cm}^{2}$, while at the same thickness the curves for $\mathrm{Na}$ and $\mathrm{Mg}$ deviate by 20 percent from proportionality. This suggests that, at $12 \mathrm{kV}$, the electron range is the factor limiting the extent of lineal extrapolation of the calibration curves, while at $25 \mathrm{kV}$, the absorption of the softel $x$-rays is the limiting factor. As a practical point, when the mass thickness of a samplt is smaller than $0.1 \mathrm{mg} / \mathrm{cm}^{2}$, even $\mathrm{Mg}$ and $\mathrm{Na}$ will be measured accurately by extrapolation o the calibration curves at $12 \mathrm{kV}$. When the mass thickness is 1 arger than $0.1 \mathrm{mg} / \mathrm{cm}^{2}$, only the heavier elements will be measured accurately, provided the accelerating voltage is appropriately adjusted to the final mass thickness. 

- $\mathrm{Na}=31.1 \%$
$\triangle \mathrm{Cl}=52.8 \%$
$\nabla P=2.1 \% \circ \mathrm{Mg}=1.6 \%$
$\mathrm{K}=2.6 \% \mathrm{H}, \mathrm{O}=9.8 \%$

$12 \mathrm{kV}$
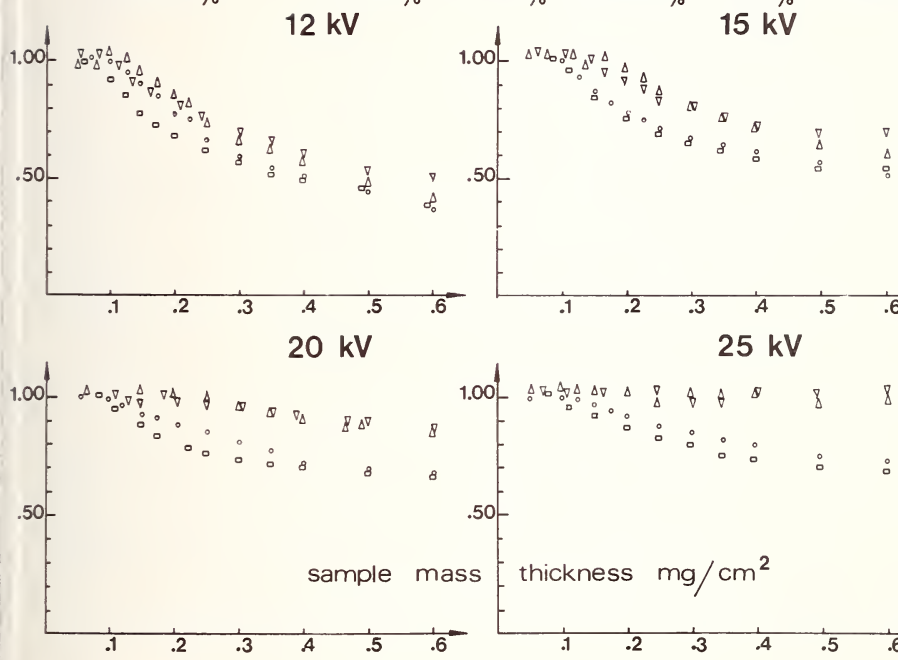

$25 \mathrm{kV}$

$15 \mathrm{kV}$
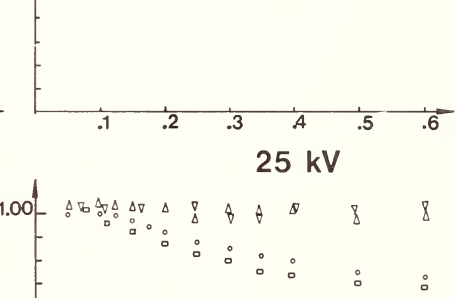

Figure 23. Lack of proportionality of the emerging intensity with the sample mass thickness. Experimental values for samples of the given composition in weight percent. Ordinate - ratio of apparent to real concentration. 

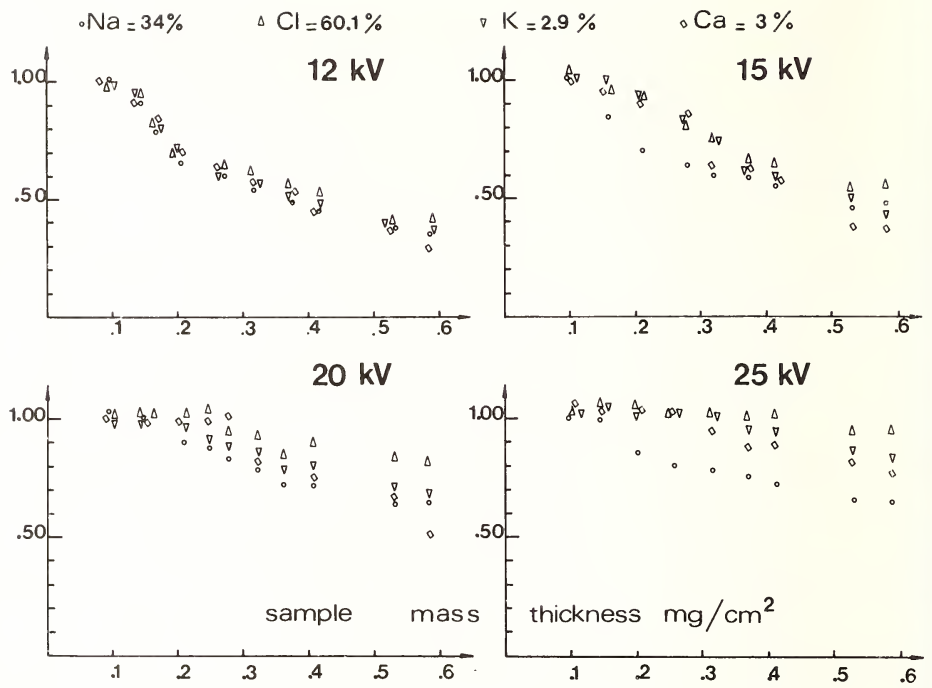

Figure 24. Lack of proportionality of the emerging intensity with the sample mass thickness. Experimental values for samples of G3 composition (weight percent). Ordinate - ratio of apparent to real concentration.

\section{Range of linearity of the calibration curves calculated for a thin film}

The calculated mass thickness of the sample has a physical signification, since the lack of linearity of the calibration curves is reproducible for a given mean mass thickness. A thin film model can therefore be considered as a theoretical model for those lyophilized samples.

The solutions examined in the preceding paragraph mainly contain $\mathrm{NaCl}$. The electron energy distribution is therefore calculated within a $\mathrm{NaCl}$ matrix and for the same voltages experimentally examined ( $12 \mathrm{kV}-15 \mathrm{kV}-20 \mathrm{kV}-25 \mathrm{kV}$ ), according to a Monte Carlo process [3] dealing with 10,000 electrons. The details of the computer program were reported earlier $[6,7]$. The electron range is divided into 20 to 50 steps, according to the accelerating voltage value, to get discrete mass thickness of approximately the same values. We define as relative intensity the ratio of the emerging intensity from a film of a given mass thickness to the intensity emerging from a $f i 1 \mathrm{~m} 0.025 \mathrm{mg} / \mathrm{cm}^{2}$, corrected for the mass thickness of the samples. The relative intensity is calculated for each element, in each solution ( $H$ and G3) and for discrete mass thickness values, and reported in figures 25 and 26 as a function of the mass thickness. These ratios are defined identically to the ratios reported in figures 23 and 24; they should be equal to one if the emerging intensity is proportional to mass thickness, i.e. proportional to the amount of element in the analyzed volume. The scales are the same for both series of graphs. Note that, qualitatively, the loss of proportionality of the emerging intensity to the mass thickness, as latter increases, is in the same order of magnitude for experimental results and for the thin film model. 


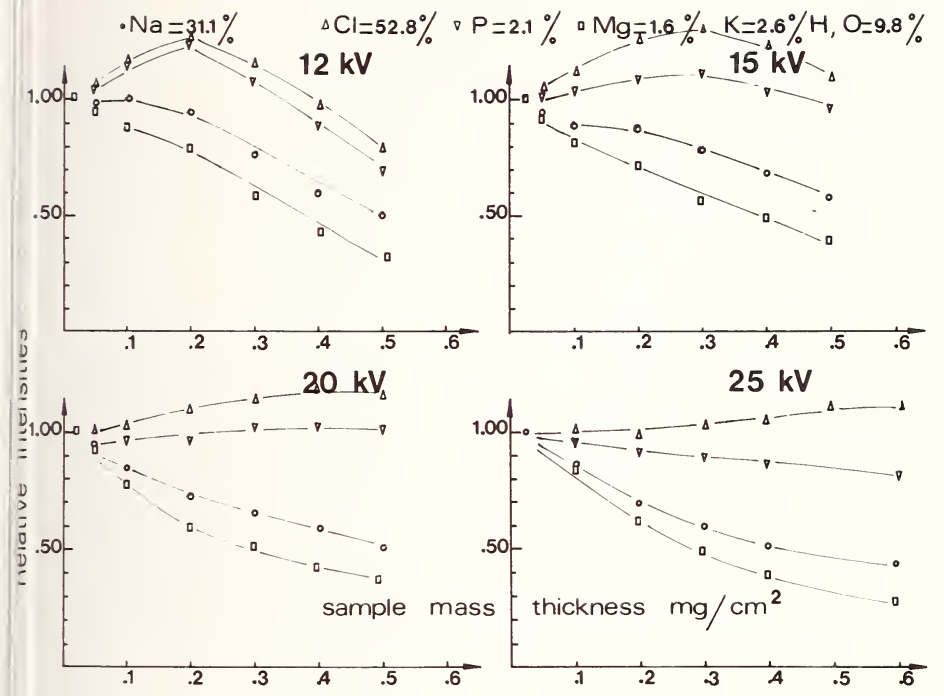

Figure 25. Lack of proportionality of the emerging intensity with the sample mass thickness. Theoretical values for a thin film. Ordinate - for definition of relative intensity, see text. 

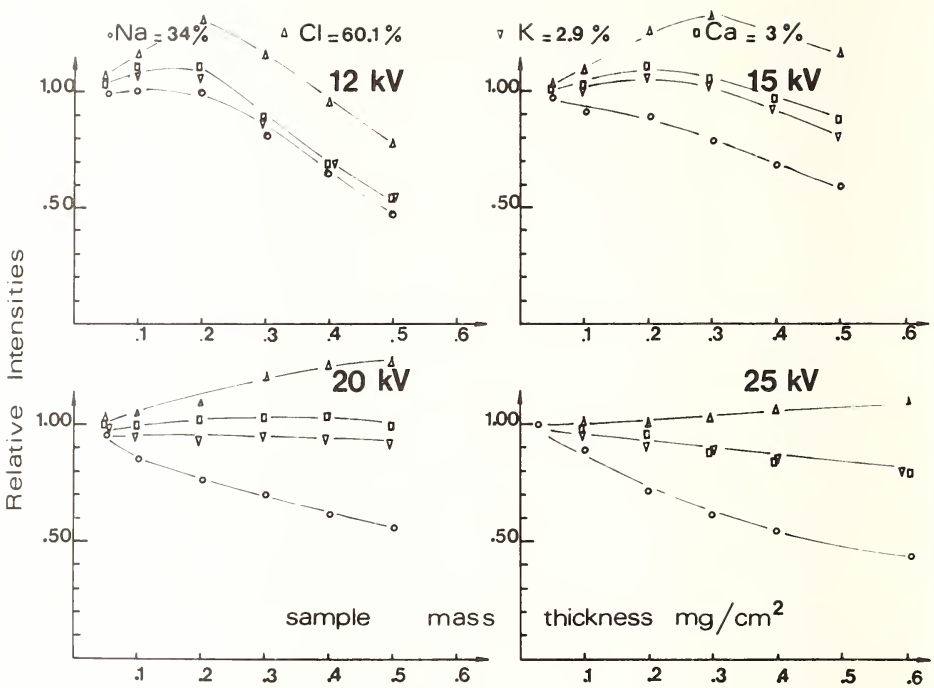

Figure 26. Lack of proportionality of the emerging intensity with the sample mass thickness. Theoretical values for a thin film of the given composition. Ordinate - for definition of the relative intensity, see text.

$H$ solution. At any voltage, both chlorine and phosphorus behave differently from sodium and magnesium. They exhibit a shoulder, characteristic of the x-ray depth distribution function, which does not appear in the experimental results. The emerging magnesium and sodium intensities decrease when the mass thickness increases, but the effect is greater for magnesium which has a higher absorption coefficient than sodium. The phosphorus and chlorine signals should be enhanced at 12 and $15 \mathrm{kV}$, contrary to what is experimentally observed, while at 20 and $25 \mathrm{kV}$, the emerging intensities should be roughly proportional to sample mass thickness up to $0.4 \mathrm{mg} / \mathrm{cm}^{2}$, which is experimentaily observed. For sodium and magnesium, the lack of proportionality of the emerging intensity with the sample mass thickness is depicted by the thin film model at 12 and $15 \mathrm{kV}$; while, at 20 and $25 \mathrm{kV}$, the lack of proportionality of the emerging intensity with the sample mass thickness is not so severe as predicted by the model.

G3 solution. Chlorine and sodium exhibit the same theoretical response as in the $H$ solution (compare figures 25 and 26 ). The potassium and calcium lines follow very closely that of sodium at $12 \mathrm{kV}$, and, when the voltage increases, fall intermediately between the chlorine and the sodium curves. For chlorine, the difference between experimental and theoretical results is the same as in the $H$ solution. For sodium, the theoretical model again predicts less absorption than that experimentally observed at 12 and $15 \mathrm{kV}$, and more absorption than that observed at 20 and $25 \mathrm{kV}$. The potassium and calcium experimental 
sults agree with the model at 20 and $25 \mathrm{kV}$, but disagree at 12 and $15 \mathrm{kV}$ where experintal results decrease more rapidly with the mass thickness than predicted by the model. ${ }^{2}$

The results from thin film model show the influence of $x$-ray absorption upon the loss proportionality of the emerging intensity as mass thickness increases. The small fferences between experimental and theoretical results can be explained by morphologic pearance of the samples - their scanning electron microscope images show piles of crystals ose size is a function of the solution composition. From these images, it seems reasonle to consider the samples as a series of units of various thickness, with a statistical stribution in thickness. Because the experimental curves do not exhibit the shoulder eoretically predicted for the heavier elements $(\mathrm{Cl}, \mathrm{K}, \mathrm{Ca}$, and $\mathrm{P})$, nor a so severe crease of the emerging intensities for the lighter elements ( $\mathrm{Na}$ and $\mathrm{Mg}$ ), it seems obable that samples contain both units of low mass thickness and those of high mass ickness, with the former in majority. The next approach to a theoretical model should gin by considering a statistical distribution of the repartition of mass thickness within e samples.

\section{Conclusion}

From this study, it appears that simply obtaining linear calibration curves linking asured intensity and concentration of an element is not sufficient to insure the accuracy electron probe analysis of fluid. In addition, we must consider identity of the degree oxidation and stability of the counting rate. Taking into account these factors permits e to achieve calibration curves whose range exceeds that normally encountered in biologi.1 specimens, particularly for $\mathrm{P}, \mathrm{Cl}, \mathrm{K}$, and $\mathrm{Ca}$ at voltage 20-25 kV. To predict the fect of matrix on the proportionality range, a theoretical model is necessary. The thin $1 \mathrm{~m}$ model is a good beginning but it needs to be improved. Among other things, the model eds to take into account the heterogeneity in mass thickness of samples, as revealed by ie secondary electron pictures. This will require more study of the distribution and ze of particulates constituting the sample. Such a theoretical model, by predicting the Ifluence of matrix on emerging intensities, will serve as a guide in the preparation of andard solutions, and should further advance the systematic application of the electron croprobe to the analysis of biological fluid.

We thank Dr. Kurt Heinrich, Dr. Rex Jamison, and Dr. Christian de Rouffignac for itical reading of our manuscript.

See paper by Dale E. Newbury et al., Monte Carlo Electron Trajectory Simulation - an Aid for Particle Analysis, this book. 
[1] Agus, Z. A., Gardner, L. B., Beck, L. H., and Goldberg, M., Am. J. Physiol., 224, 1143 (1973).

[2] Beeuwkes, R., Amberg, J. M., and Essandoh, L., Kidney Intern., 12, 438 (1977).

[3] Bishop, H., Thesis, Cambridge University (1966).

[4] Faessler, A. Von and Goehring, M. , Naturwiss, 39, 169 (1952).

[5] Garland, H. 0., Hopkins, T. C., Henderson, I. W., Haworth, C. W., and Chester-Jones, I., Micron, $\underline{4}, 164$ (1973).

[6] Henoc, J. and Maurice, F., Rapport CEA n 4615 (1975).

[7] Henoc, J. and Maurice F., NBS Special Publication 460 (1976).

[8] Ingram, M. J. and Hogben, G. A., Analyt. Bioch., 18, 54 (1967).

[9] Lechene, C., Proc. 5th Nat. Conf. Electron Probe Analysis, New York, 32 A (1970).

[10] Lechene, C., in Microprobe Analysis as Applied to Cells and Tissues (Academic Press, London, 1974) p. 351 .

[11] More1, F. and Roine1, N., J. Chim. Phys. et Phys. Chim., 66, 1084 (1969).

[12] Morel, F., Roinel, N., and Le Grimellec, C., Nephron, $\underline{6}, 350$ (1969).

[13] Quamme, G. and Dirks, J., Can. J. of Spectroscopy, 20, 38 A (1975).

[14] Quinton, P., Proc. 10th Nat. Conference of the Microbeam Analys is Society, Las Vegas, 50A (1975).

[15] Quinton, P., Proc. of the Scanning Electron Microscopy Meeting, Los Angeles, Vol. II, 391 (1978).

[16] Ramsay, J. A., Brown, R. H. B. J., and Crogham, P. C., J. Exp. Biol., 32, 822 (1955).

[17] Rick, R., Horster, M., Dörge, A., and Thurau, K., Pflügers Archiv. , 369, 95 (1977).

[18] Roinel, N., Richard, J. P., Robin, G., and Morel, F., J. Microscopie, 18, 285 (1973).

[19] Roine1, N., J. Microscopie Biol. Cell., 22, 261 (1975).

[20] Roinel, N., Proc. of the XXVIIth International Congress of Physiological Sciences, XII, 144 (1977). 
ational Bureau of Standards Special Publication 533. This Publication Originated from a pecial Session on Particle Analysis, 13th Annual bciety, Ann Arbor, MI, June 22, 1978. (Issued April 1980)

APPLICATION OF AUGER-ELECTRON SPECTROSCOPY AND X-RAY PHOTOELECTRON SPECTROSCOPY TO THE CHARACTERIZATION OF POLLUTANT PARTICLES ${ }^{1}$

\section{J. Powel1}

National Bureau of Standards

Surface Science Division

Washington, D. C. 20234

A short review is given of the use of Auger-electron spectroscopy (AES) and $x$-ray photoelectron spectroscopy (XPS) for surface characterization of pollutant particles from the atmosphere. The significance of the surface composition is emphasized with reference to catalytic transformations of gaseous $\mathrm{SO}_{2}$ and $\mathrm{NO}_{x}$ and to the possible adverse effects on human health of sulfates, toxic trace elements, and carcinogenic polycyclic aromatic matter. AES and XPS have been used to identify elements present on particles and the variation of composition with depch. XPS has been used to identify the chemical state of surface species, to identify toxic trace elements, and to study catalytic reactions involving gaseous pollutants. Areas where further work and developments are needed are (1) kinetic measurements for the significant heterogenous transformations of gaseous pollutants on particle surfaces, (2) analyses of aerosol and particle surfaces under ambient atmospheric rather than vacuum conditions, and (3) surface analysis of selected individual particles.

\section{Introduction}

Over the past two decades there has been considerable public concern over pollution of he atmosphere. Attention was focussed initially on gaseous pollutants, but it is now stablished that air pollution by particulate matter is also a serious problem [1-3] ${ }^{2}$. Pariculate pollutants from coal-fired power plants have been shown to have adverse effects on Iuman health, vegetation, and materials [1-3]. Since this nation and others will depend on nergy generated at coal-fired power plants in the next several decades to a greater extent han now, it is clear that the properties of pollutant particles and the nature of reactions of gaseous pollutants on particles need to be better understood. Particulate emissions from ther sources (asbestos materials, automobiles, industrial furnaces) may also be significant $1-3]$.

This article is a brief review of the applications of Auger-electron spectroscopy (AES) and $x$-ray photoelectron spectroscopy (XPS) or electron spectroscopy for chemical analysis ESCA) for the characterization of the surface regions of particulate pollutants from the itmosphere. The significance of surface characterization is described in Section 2, and the characteristics of AES and XPS are summarized in Section 3. Applications of AES and XPS for sarticle characterization are described in Section 4; more detailed descriptions of the use If AES and XPS for surface analyses of pollutant particles are given in two recent review articles [4-5]. Finally, in Section 5, there is a discussion of outstanding problems and a description of several recent instrumental developments which could be useful in future investigations.

${ }^{1}$ Work carried out at the National Bureau of Standards under the sponsorship of the U.S Department of Energy Division of Biomedical and Environmental Research.

${ }^{2}$ Figures in brackets indicate the literature references at the end of this paper. 
A specialist group has calculated the probability of deposition of particles in different regions of the human respiratory tract as a function of particle diameter [6]. For particle diameters greater than $10 \mu \mathrm{m}$, approximately 80 percent or more of the particles are deposited in the nasopharyngeal compartment. Deposition in the tracheobronchial region has a low probability $(<10 \%)$ almost independent of particle size for diameters greater than $0.1 \mu \mathrm{m}$; the probability does increase to 30 percent for a particle diameter of $0.01 \mu \mathrm{m}$. The probability of deposition in the pulmonary compartment increases from about 10 percent for a particle diameter of $10 \mu \mathrm{m}$ to 60 percent for a diameter of $0.01 \mu \mathrm{m}$. Particles with diameters less than $10 \mu \mathrm{m}$ can therefore be inhaled deeply into the human lungs and have to be considered in epidemiological studies. Although the actual mechanism of deposition is primarily dependent upon the particle size, the shape of a particle can also affect the efficiency of its deposition [6]. The size range of pollutant particles in the atmosphere to be considered are, first, the so-called primary particles with diameters typically between 1 and $20 \mu \mathrm{m}$ which are characteristic of an emission source and, second, the secondary particles with diameters less than $1 \mu \mathrm{m}$ which are produced as a result of atmospheric reactions [1].

An analysis of the recent literature concerned with investigations of particle pollutants in the atmosphere yielded the following conclusions [7].

(1) Smaller particles have longer residence times in the atmosphere.

(2) Fine particles, of diameter less than $1 \mu \mathrm{m}$, can be transported hundreds to thousands of kilometers downward from their point of formation and, because of their high surface/volume ratio, are capable of adsorbing significant quantities of toxic gases.

(3) Toxic elements such as $\mathrm{Pb}, \mathrm{Cd}, \mathrm{Tl}, \mathrm{V}$, and $\mathrm{Ni}$ have higher relative concentrations in smaller particles.

(4) Sulfur dioxide can be catalytically converted to sulfate species on particle surfaces.

(5) Fine particles can act as transport vehicles for adsorbed and reacted gaseous pollutants; synergistic effects on human health are significant [1].

(6) Polycyclic aromatic matter (PAM) is formed in combustion processes and is condensed on particle surfaces; some PAM is carcinogenic.

The nature of the surfaces of pollutant particles and, in particular, the surface composition are important for the following reasons $[4,5,7]$.

(a) Gaseous pollutants in the atmosphere such as $\mathrm{SO}_{2}$ and $\mathrm{NO}_{x}$ can be catalytically converted to sulfate and nitrate species.

(b) Toxic elements can be located in greater relative concentrations in the surface region of a particle than in the bulk.

(c) Carcinogenic PAM may be on the surface.

(d) The surface composition of a particle inhaled into the human respiratory system may have a greater effect on health than the average composition for the entire particle. 
Specific questions which should then be addressed in the characterization of particle faces are [7]:

(i) To what extent is the surface composition different from the bulk composition?

(ii) To what extent is the surface enriched in toxic elements or carcinogenic molecules?

(iii) How does the surface composition correlate with particle size (and, perhaps, with method of formation and subsequent history)?

(iv) What are the reaction products and rates for various gases and gas mixtures interacting with particles?

\section{Surface Analysis by AES and XPS}

There are four techniques presently in common use for surface analyses of a great iety of samples: AES, XPS, secondary-ion mass spectroscopy (SIMS), and ion-scattering ectroscopy (ISS) [8]. The application of SIMS to particle characterization is described a companion article by Newbury but, to the author's knowledge, there has been no appliiion in this area of ISS.

The surface sensitivity of AES and XPS is due to the very short inelastic mean free th (IMFP), typically 5 to $20 \AA$, of the electrons of interest in the sample [9]. The formation volume, that region of the sample from which most of the detected electrons iginate, is a function of the measurement configuration as well as properties of the mple (primarily the IMFP). For the particulate samples of interest here, the information lume is very difficult to define because of the structural complexities which can be sected and the likely lack of chemical homogeneity of the information volume for a parcular measurement. Scanning electron micrographs of coal-derived fly-ash samples from ectrostatic precipitators, for example, show a great variety of structural forms [10].

Ideally, it would be desirable to examine the surface composition of different dividual particles. In practice, tradeoffs have to be made between spatial resolution, ssitivity for the detection of trace elements, and damage to the sample during the course the measurement. Commercial AES instrumentation is now available with which submicroter spatial resolution can be obtained. For particulate samples of possibly poor elecical and thermal conductivities, charging, and electron-beam-induced damage [11] can se severe problems if high spatial resolution was attempted. For XPS, damage by the ray beam is believed to be minor, but the spatial resolution achievable with commercial strumentation is typically a few millimeters. Sensitivity for the detection of trace ements can be improved, in principle, by increasing the measurement time but, in practice, a potential gain is limited by beam-damage effects, adsorption on the sample of moleles from the ambient vacuum, and the stability of the equipment.

\section{Applications of AES and XPS for Particle Characterization}

A brief description will be given here of applications of AES and XPS for surface aracterization of particles collected from power plants and the atmosphere. This descripon is intended to be a guide to the relevant literature and to the nature of the AES and $S$ investigations rather than a comprehensive summary or review $[4,5]$.

T. Novakov has pioneered in demonstrating that XPS is useful for particulate characrization, in speciation studies of particles, and in studies of heterogenous reactions particle surfaces [4]. He and his coworkers have been instrumental in showing the portance of surface characterization of pollutant particles for the atmosphere and the wer of XPS (and, by implication, other methods of surface analysis) for characterizing rticle surfaces and surface processes.

Cheng et a1. [12] have used AES to characterize fly ash from the stacks and precipitars of both coal-fired and oil-fired power plants. The Auger spectrum for the sample from 
the 0i1-fired plant showed a strong vanadium signal in addition to peaks for $\mathrm{C}, \mathrm{S}, 0$, and $\mathrm{Mg}$. The AES data for the sample from the coal-fired plant showed structure due to C, S, $0, \mathrm{Ca}, \mathrm{N}, \mathrm{Fe}$, and $\mathrm{Na}$.

Linton et a1. [13] have measured AES depth profiles, i.e., changes in the relative strengths of different elemental signals as the surface was eroded by sputtering, for fly ash samples collected from coal-fired power-plant stacks. The signals due to $\mathrm{K}$, $\mathrm{Na}$, and $S$ were enhanced in a surface region of approximately $30 \AA$ depth compared to those for deeper regions while the signals due to $\mathrm{Fe}, \mathrm{Al}$, and Si did not vary appreciably with depth.

Lindfors and Hovland [14] have used both AES and XPS to detect elements present on the surface of fly ash from the precipitator of a coal-fired power plant. These authors detected $\mathrm{Al}, \mathrm{Si}, \mathrm{K}, \mathrm{C}, \mathrm{O}, \mathrm{Fe}, \mathrm{Na}, \mathrm{Ca}, \mathrm{S}, \mathrm{Cl}$, and $\mathrm{N}$ on individual particles from different parts of the precipitator, with some elements being present in one region and not in the other. Many of the elements were detected also by XPS analysis of an assemblage of particles. AES depth profiles for a particle showed a predominance of Si and 0 while XPS depth profiles for an assemblage from the precipitator outlet showed surface enrichment of $C, S$, and $\mathrm{Na}$ and surface depletion of $\mathrm{Al}, \mathrm{Fe}$, and $\mathrm{Ca}$.

Keyser et a1. [5] have reported AES and SIMS depth profiles for large (>10 $\mu \mathrm{m})$ automobile exhaust particles. The AES measurements showed an appreciable surface enrichment of $\mathrm{Pb}$ and $\mathrm{Br}$ and $\mathrm{a}$ lesser enrichment of $\mathrm{S}$.

XPS has been used by Hulett and coworkers in several investigations of fly-ash particles. In one study, fly-ash samples from the precipitators of several power plants were analyzed and it was found that the AI and Si XPS signals were enhanced, while the S signal due to sulfate species was diminished after the samples were leached with rainwater to simulate the effects of rain [15].

Grieger [16] has collected particle samples from urban, suburban, and rural areas both 1 day and 14 days after rain. XPS measurements showed that the particles from the rural area had appreciably smaller concentrations of $\mathrm{Pb}, \mathrm{S}$, and $\mathrm{N}$ than the other areas, and that the suburban area had a lesser amount of $\mathrm{Pb}$ than the urban area but comparable amounts of $S$ and $N$. Rain washed away some of the $N$ and $S$ compounds.

Novakov and coworkers have performed many XPS studies of particle samples from the atmosphere [4]. In an early paper [17], the small "chemical shifts" of XPS lines for $\mathrm{N}$ and $\mathrm{S}$ were analyzed to give information on the relative amounts of nitrate-, ammonium-, amino-, and pyridine-nitrogen compounds and of sulfate and sulfite compounds on aerosols of different sizes and collected at different times during a day in Pasadena. Further XPS work [18]. showed the presence of seven separate chemical species of sulfur $\left(\mathrm{SO}_{3}, \mathrm{SO}_{4}{ }^{2}\right.$, $\mathrm{SO}_{2}, \mathrm{SO}_{3}{ }^{2}, \mathrm{~S}$, and two sulfides) on atmospheric aerosols. Chang and Novakov [19] conducted laboratory experiments in which $\mathrm{NO}$ and $\mathrm{NH}_{3}$ were reacted with soot and graphite at various temperatures. XPS measurements showed the presence of ammonium complexes at ambient temperatures while amines, amides, and nitriles were produced at higher temperatures. Comparisons of this data with XPS measurements of aerosol particles led to the assignment of three nitrogen features as being due to ammonium, amine and/or amide, and nitrile species. A similar XPS investigation [20] involving the reaction with $\mathrm{SO}_{2}$ with soot showed that soot particles in the atmosphere can play a major role in the catalytic conversion of $\mathrm{SO}_{2}$ to sulfate species.

Barbaray et a1. [21] have used XPS to study the oxidation of $\mathrm{SO}_{2}$ over $\mathrm{V}_{2} \mathrm{O}_{5}$ particles and carbon. There was little direct interaction between $\mathrm{SO}_{2}$ and $\mathrm{V}_{2} \mathrm{O}_{5}$ for temperatures below $150{ }^{\circ} \mathrm{C}$, but at $200{ }^{\circ} \mathrm{C}$ oxidation proceeded to adsorbed sulfate and sulfur trioxide. In the presence of $\mathrm{NO}_{2}$ or $\mathrm{H}_{2} \mathrm{O}$, however, sulfate is formed at $25{ }^{\circ} \mathrm{C}$.

XPS has been used by Campbell et al. [22] to examine a fly ash designated SRM 1633 by the National Bureau of Standards. These authors determined the oxidation states of a number of elements and found that the concentrations of $\mathrm{Na}, \mathrm{C}, 0,0$ and $\mathrm{S}$ decreased and the concentrations of $\mathrm{Si}, \mathrm{Al}$, and $\mathrm{Fe}$ increased when approximately $50 \AA$ of the sample was removed by sputtering. 
Allegrini and Mattogno [23] have examined particulate emissions from several sources ith XPS. They have identified the chemical states of $S$ and $N$ in the samples from the chemcal shifts of the elemental XPS lines.

Finally, Jach et al. [24] have made XPS analysis of several fly-ash and urban dust amples. By accumulating data with a multichannel analyzer over limited spectral regions nd with multiple sweeps, these authors were able to detect some 30 elements, some of which ere not detected in "bulk" analyses by neutron activation. Amongst the elements detected n a sample from one source were Si, Al, K, Fe, Ca, Ti, $\mathrm{Na}, \mathrm{As}, \mathrm{Co}, \mathrm{Bi}, \mathrm{Cl}, \mathrm{Sr}, \mathrm{Ba}, \mathrm{Zn}, \mathrm{V}$, $r, \mathrm{P}, \mathrm{Sb}, \mathrm{Mo}, \mathrm{Pb}$, and $\mathrm{Ni}$.

\section{Concluding Remarks}

A description has been given of recent applications of AES and XPS to determine the urface composition of pollutant particles from the atmosphere. These techniques have the ensitivity to detect elements present in the outermost $\approx 10 \AA$ of a solid sample and, by emoval of surface atoms by sputtering, to determine the variation of composition with epth. AES has the capability to measure individual particles although it has yet to be emonstrated that this can be done routinely without significant damage to the sample.

The surface composition of pollutant particles is important for several reasons. First, aseous pollutants such as $\mathrm{SO}_{2}$ and $\mathrm{NO}_{x}$ can be catalytically converted to sulfate and nitrate

pecies on the particle surfaces. Sulfates, in particular, have been associated with dverse health effects and damage to vegetation and materials. Second, toxic elements ay be located in the surface region of a particle to a greater relative concentration than he bulk. Third, carcinogenic polycyclic aromatic matter (PAM) may be in the particle urface. The amounts of toxic elements and PAM on inhaled particles need to be known for ealth-effects studies.

AES and XPS have been used to identify elements present on particle surfaces and to etermine the variation of composition with depth. XPS has been used for the identification $f$ chemical species, the detection of toxic trace elements, and the study of catalytic onversions of gaseous pollutants. These studies have demonstrated the basic feasibility $f$ the approach and need to be extended to the examination of pollutant particles from ifferent regions and to the acquisition of kinetic data for the more important reactions.

There are two areas where improvements in measurement capability would be valuable. irst, surface analyses by AES and XPS need to be made with the sample in an ultrahigh acuum. Volatile species can be lost from the sample surface during pumpdown, although this an be minimized by appropriate cooling of the sample [19]. It would be very desirable 0 be able to measure surface composition, with a sensitivity and specificity comparable to hat of AES and XPS, of aerosols and particles at atmospheric pressure. One approach is o use particle-induced x-ray emission (PIXE) with MeV proton beams brought from an ccelerator vacuum through a thin window to a sample in a helium environment at or near tmospheric pressure $[25,26]$. Light elements cannot be detected by PIXE, but protonlastic-scattering can be used for this purpose [25]. Although PIXE does not have the urface sensitivity of AES and XPS, it could be used to detect changes in elemental concenrations as environmental or reaction conditions (partial pressure of $\mathrm{SO}_{2}$ or $\mathrm{NO}_{\mathrm{x}}$, humidity, emperature, etc.) are varied. An alternative approach is to use a combination of $x$-ray nd electron spectroscopies that may be feasible in the near future as more intense sources f variable-energy $x$-rays become available [27].

A second area where instrumental improvements would be desirable is in surface nalyses of selected individual particles. Analyses of this type can now be performed by ES and SIMS, but beam-damage effects may limit the scope of the analysis (e.g., the detecion of trace elements). Several techniques could be useful in the future. First, the damage products" of electron bombardment, namely ions, could be detected as in SIMS [28]. econd, the scanning XPS system proposed by Cazaux [29] and demonstrated by Hovland [30] ould be ideally suited for XPS analyses of selected particles; this technique has a patial resolution of about $10 \mu \mathrm{m}$. Finally, the photoelectron microscope developed by iriffith et al. [31] can detect $\approx 10 \mu \mathrm{m}$ particles. By varying the wavelength of the xciting ultraviolet radiation, it might be possible to detect and selectively image urface 7 igands with differing work function or ionization potential. 
[1] Burchard, J. K., J. Air Pollution Control Assoc. 24, 1141 (1974); ibid. 25, 99 (1975); Harrington, R.E., J. Air Pollution Control Assoc. 24, 927 (1974); Fennelly, P. F., J. Air Pollution Control Assoc. 25, 697 (1975).

[2] Ferris, B. G., J. Air Pollution Control Assoc. 28, 482 (1978); Lave, L. B., and Seskin, E. P., American Scientist 67, 178 (1979).

[3] Rauch, R. J., Chemical and Engineering News 57, 25, 47 (1979); Crandal1, R. W., ibid. 57, 29, 51 (1979); Barber, W. C., ibid. 57, 34 (1979); Lave, L. B., and Seskin, E. P., ibid. 57, 38, 49 (1979); Comar, C. L., ibid. 57, 42, 48 (1979).

[4] Novakov, T., Chang, S. G., and Dod, R. L., in Contemporary Topics in Analytical and Clinical Chemistry, edited by D. M. Hercules, G. M. Hieftze, L. R. Snyder, and M. A. Evenson (Plenum Press, New York, 1977), Volume 1, p. 249.

[5] Keyser, T. R., Natusch, D. F. S., Evans, Jr., C. A., and Linton, R. W., Environ. Sci. and Tech. 12, 768 (1978).

[6] Air Quality Criteria for Particulate Matter, National Air Pollution Control Administration Publication No. AP-49 (U. S. Department of Health, Education, and Welfare, Washington, D. C., January, 1969), Chapter 9.

[7] Powe11, C. J., Surface Composition of Particulate Pollutants in the Atmosphere, unpublished Issue Study, May 1976.

[8] Powe11, C. J., Applications of Surf. Science 1, 143 (1978).

[9] Powe11, C. J., Surf. Science 44, 29 (1974).

[10] Fisher, G. L., Chang, D. P. Y., and Brummer, M., Science 192, 553 (1976).

[11] Isaacson, M. S., in Principles and Techniques of Electron Microscopy, edited by M. A. Hayat (Van Nostrand Reinhold Co., New York, 1977), Volume 7; T. E. Madey and J. T. Yates, Jr., to be published.

[12] Cheng, R. J., Mohnen, V. A., Shen, T. T., Current, M., and Hudson, J. B., J. Air Pollution Control Assoc. 26, 787 (1976); Menotti, F., Hudson, J. B., Cheng, R. J., and Mohnen, V. A., in Proceedings of the Conference on Carbonaceous Particles, Berkeley, Calif., March 20-22, 1978 (Lawrence Berkeley Laboratory Report), edited by T. Novakov, to be published.

[13] Linton, R. W., Loh, A., Natusch, D. F. S., Evans, Jr., C. A. and Williams, P., Science 191, 852 (1976); Linton, R. W., Williams, P., Evans, Jr., C. A., and Natusch, D. F. S., Anal. Chem. $\underline{49}, 1514$ (1977).

[14] Lindfors, P. A., and Hovland, C. T., in Environmental Pollutants, Detection and Measurement, edited by T. Y. Toribara, J. R. Coleman, B. E. Dahneke, and I. Feldman (Plenum Press, N. Y., 1978), p. 349.

[15] Hulett, L. D., Dunn, H. W., Dale, J. M. , Emery, J. F., Lyon, W. S., and Murty, P. S., in Measurement, Detection and Control of Environmental Pollutants, Proceedings of a Symposium, Vienna, 15-19 March 1976 (International Atomic Energy Agency, Vienna, 1976), p. 29.

[16] Grieger, G. R., Am. Laboratory 8, 77 (Apri1, 1976).

[17] Novakov, T., Mueller, P. K., Alcocer, A. E., and Otvos, J. W., J. Colloid Interface Sci. 39,225 (1972). 
[18] Craig, N. L., Harker, A. B., and Novakov, T., Atmos. Environment $\underline{8}, 15$ (1974).

[19] Chang, S. G. , and Novakov, T., Atmos. Environment $\underline{9}, 495$ (1975).

[20] Novakov, T., Chang, S. G., and Harker, A. B., Science 186, 259 (1974).

[21] Barbaray, B., Contour, J. P., and Mouvier, G., Atmos. Environment 11, 351 (1977); Environmental Sci. and Tech. 12, 1294 (1978).

[22] Campbe11, J. A., Smith, R. D., and Davis, L. E., App1. Spectrosc. 32, 316 (1978).

[23] Allegrini, I., and Mattogno, G., Sci. Total Environ. 9, 227 (1978).

[24] Jach, T. J., Powel1, C. J., Smal 1, J. A., and Newbury, D. E. (to be published).

[25] Ziegler, J. F., Physics Today 29, 52 (November, 1976).

[26] Deconninck, G., Nuc]. Instr. Methods 142, 275 (1977).

[27] Powe11, C. J., J. Vac. Sci. Tech. 15, 549 (1978).

[28] Joshi, A., and Davis, L. E., J. Vac. Sci. Tech. 14, 1310 (1977).

[29] Cazaux, J., Rev. Phys. Appl. 10, 263 (1975).

[30] Hovland, C. T., in Proceedings of the Seventh International Vacuum Congress and the Third International Conference on Solid Surfaces, edited by R. Dobrozemsky, F. Rudenauer, F. P. Viehböck, and A. Breth (R. Dobrozemsky et al., Vienna, 1977), Vol. 3, p. 2363.

[31] Dam, R. J., Kongslie, K. F., and Griffith, D. H., Photochemistry and Photobiology 22, 265 (1975). 
tional Bureau of Standards Special Publication 533. This Publication Originated from a ecial Session on Particle Analysis, 13th Annual Conference of the Microbeam Analysis ciety, Ann Arbor, MI, June 22, 1978. (Issued April 1980)

\title{
SECONDARY ION MASS SPECTROMETRY FOR THE ANALYSIS OF SINGLE PARTICLES
}

\author{
Dale E. Newbury \\ National Bureau of Standards \\ Center for Analytical Chemistry \\ Washington, D. C. 20234
}

\section{Introduction}

When particle aggregates are examined by means of electron probe microanalysis, it is equently observed that the compositions of individual particles vary markedly, even when e sample originates from a single source $(1)^{1}$. In examining a single micrometer-sized rticle by electron beam excitation, virtually all of the particle is sampled and the mposition so determined is an average over the sampled volume. The next level of inquiry to consider possible heterogeneity within the particle itself, i. e., to determine the crostructure of the particle. When the sampling volume by electron excitation is a all fraction of the particle volume, heterogeneities in the composition from place to ace in a single particle are frequently observed. Another possible microstructural ature of interest is the distribution of constituents with depth in a particle. That does the surface differ in composition from the interior? The electron interaction lume is of the order of $1 \mu^{3}$, with a sampling depth of $1 \mu \mathrm{m}$ or greater, which makes parate analysis of the surface and the interior virtually impossible. To answer questions ch as this, the technique of secondary ion mass spectrometry offers special capabilities $r$ particle analysis.

Secondary ion mass spectrometry (SIMS) has evolved into a powerful technique for the alysis of a wide range of specimens of interest in the physical and biological sciences $d$ in various technological applications $(2,3,4)$. In the SIMS technique, a beam of enertic ions is used to bombard a target, causing the process of sputtering, in which indivual atoms or clusters of atoms lying near the surface are ejected. A fraction of the uttered particles, the so-called secondary ions, is ejected in a charged state. The condary ions are collected and mass analyzed in a magnetic spectrometer or a quadrupole ss filter. Instrumentation for SIMS has been described extensively in the literature ,5).

Because of the shallow sampling depth of the secondary ion signal, which originates om a depth of about 1-2 nm, SIMS is capable of obtaining information from the surface a target. Along with Auger electron spectroscopy (AES), x-ray photoelectron specoscopy (XRS), and ion scattering spectroscopy (ISS), SIMS is considered one of the major rface analysis techniques (5). In addition, since the primary ion beam can be focussed form a small probe on the sample, restricting the region sampled to an area with dimenons in the micrometer range, SIMS can also be used as a microanalysis technique, with a nimum sampling volume of $0.01 \mu^{3}$.

SIMS offers a number of advantages for the study of individual particles, including e capabilities for detection of all elements, isotope ratio measurements, compound tection, depth profiling, and ion imaging of specific constituents. Each of these pabilities will be described in this paper with special reference to particulate studies.

igures in brackets indicate the literature references at the end of this paper. 
Examination of positive and negative secondary ion spectra from a single urban air particulate, figure 1, reveals the potential of SIMS for characterizing a complicated, multi-element target. All elements in the particle can be detected by SIMS, including hydrogen. The peaks are characterized by a high signal-to-background ratio. In the case of a major element, the peak-to-background ratio can of ten exceed $10^{6}: 1$ per nanoamp of primary ion current. (Note that the background is often independent of the beam current, so that an increase in the beam current can increase the peak counting rate without substantially affecting the background.) This high peak-to-background is observed for many elements, and as a result the SIMS technique can be used to realize very low values for the minimum detection limit. The high sensitivity per unit current makes possible the use of finely-focussed primary ion beams. Useful secondary ion spectra of particles can be obtained from targets as small as $1 \mu \mathrm{m}$ with existing instruments. Improvements in source brightness and ion optics should extend this limit to $0.1 \mu \mathrm{m}$ in the future.

\section{Detection Limits}

In considering the minimum detection limit, two factors must be taken into account: (1) the characteristics of the species of interest and (2) the matrix in which it resides. The minimum detection limit varies considerably from one element to another. The secondary ion signal is a strong function of the electronic character of the surface region from which the secondary ions are emitted. Under bombardment with oxygen primary ions, those elements which tend to react strongly with oxygen produce the highest relative signals in the positive secondary ion spectrum. Andersen (6) has demonstrated that the range of sensitivity for the pure elements is as great as six orders of magnitude, with gold the least sensitive, and elements such as aluminum and silicon the most sensitive. When several elements are dispersed in a common, oxygen-rich matrix, such as a silicate, the range of relative sensitivity is somewhat compressed. The range of the secondary ion yields becomes smaller, and therefore the minimum detectable limit shows less variation. In Table 1, a list is compiled of the minimum detectable limit for a variety of elements in a lead-silicate matrix. For the conditions of beam current employed, which were appropriate to microanalysis with a beam size of approximately $2 \mu \mathrm{m}$, the absolute detection limit is of the order of 1 part per million for many elements in the absence of interferences. Since many particulates, especially those produced by natural processes, tend to be highly oxidized, the detection limits given in Table 1 are likely to be appropriate to the analysis of unknowns. 


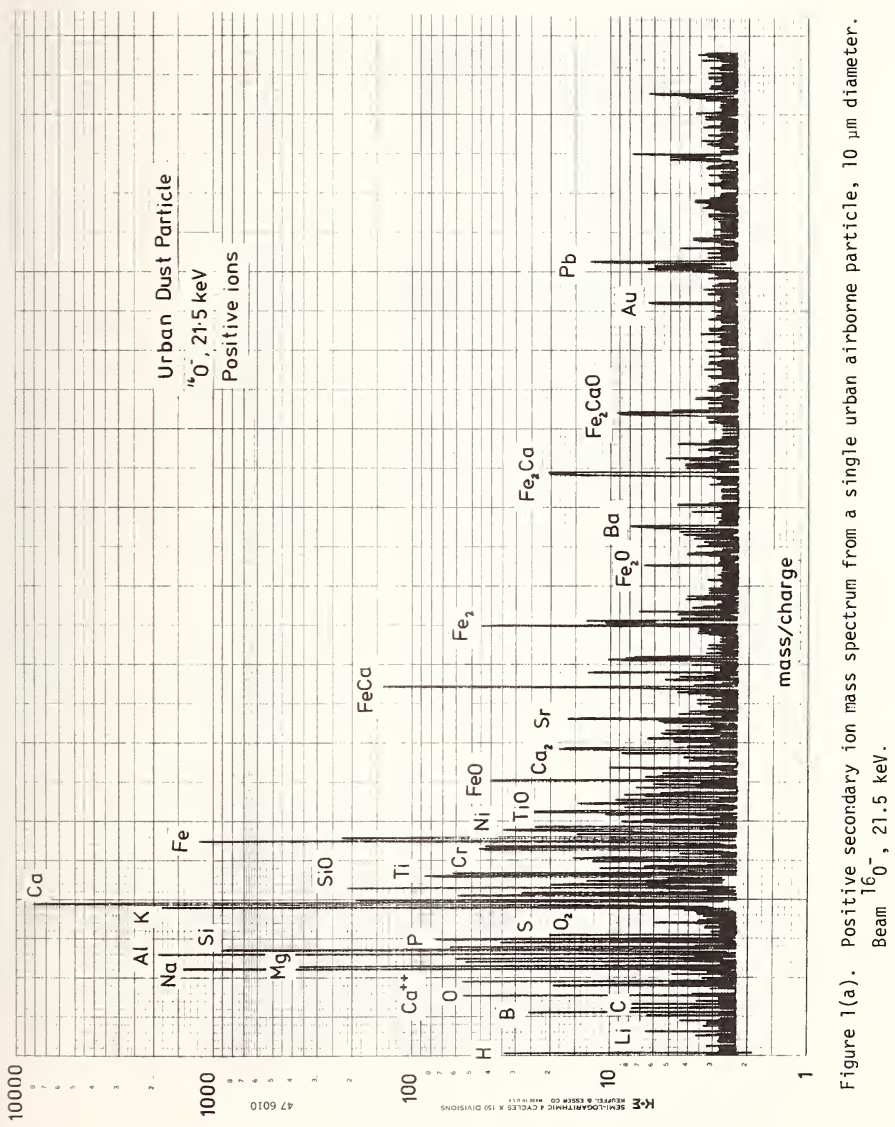




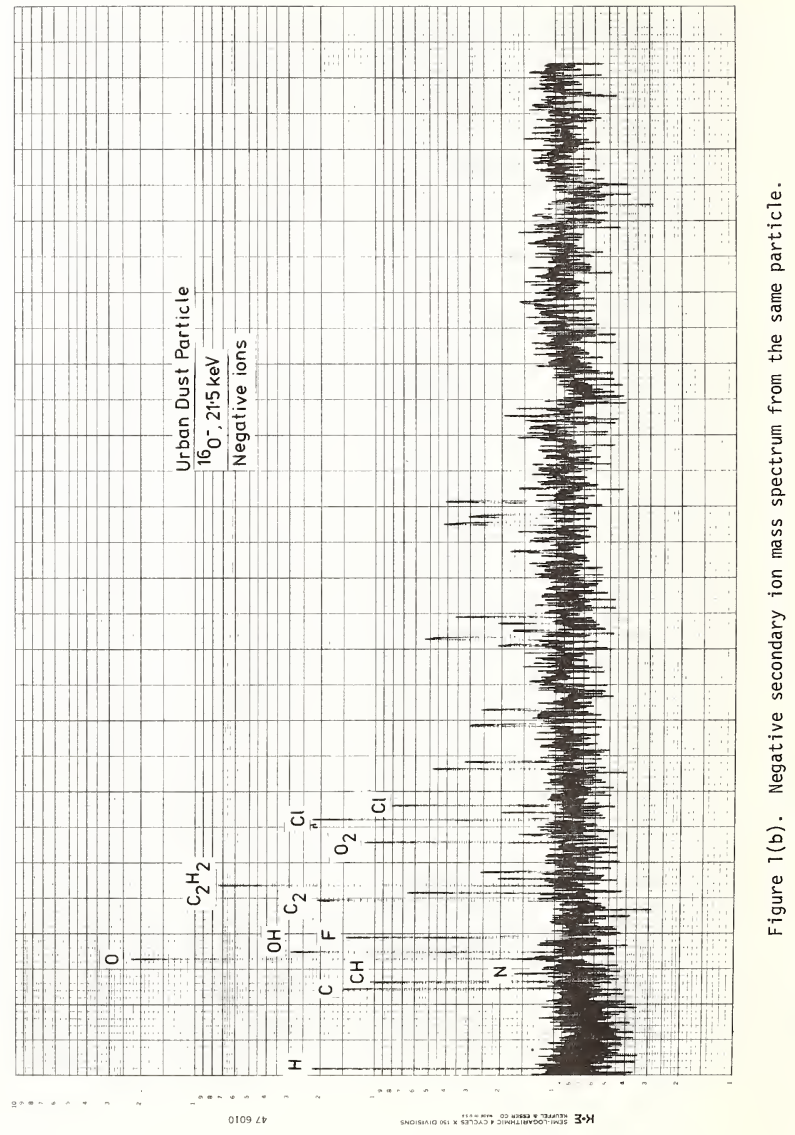


Table 1. Detection Limits in an Oxygen-Rich Matrix

$\begin{array}{cc}\text { Element }^{\mathrm{a}} & \text { Detection Limi } \\ \mathrm{Li} & 0.4 \mathrm{ppm} \\ \mathrm{B} & 6 \\ \mathrm{Mg} & 0.6 \\ \mathrm{Al} & 0.5 \\ \mathrm{P} & 23 \\ \mathrm{Ti} & 0.6 \\ \mathrm{Cr} & 1 \\ \mathrm{Fe} & 2 \\ \mathrm{Ni} & 3 \\ \mathrm{Gc} & 4 \\ \mathrm{Zr} & 0.7 \\ \mathrm{Ba} & 4 \\ \mathrm{Ce} & 2 \\ \mathrm{Eu} & 5 \\ \mathrm{Ta} & 12 \\ \mathrm{Th} & 2 \\ \mathrm{U} & 2\end{array}$

\footnotetext{
${ }^{\mathrm{a}} \mathrm{A} 11$ elements dispersed as solutes in a glass matrix of $\mathrm{Si}-\mathrm{Pb}-0$.

Detection limit for a $1 \mathrm{nA}{ }^{16} 0^{-}$ion beam and a $1 \mathrm{sec}$ integration time taken as the concentration expected to produce three times the background count rate.
}

\section{Quantitative analysis}

Practical quantitative elemental analysis of particles by SIMS can proceed from two distinct approaches: (1) empirical analysis, based on the use of relative elemental sensitivity factors derived from standards and (2) analysis based on a physical model of secondary ion formation $(3,7)$.

Sensitivity factor analysis

A relative elemental sensitivity factor can be defined as:

$$
S_{x / R}=\frac{\left(i_{x} / C_{x} f_{x}\right)}{\left(i_{R} / C_{R} f_{R}\right)}
$$

where $i$ is the measured secondary ion current, $C$ is the atomic concentration, $f$ is the isotopic abundance of the line measured, $x$ denotes the element of interest and $R$ denotes a reference element. Relative elemental sensitivity factors are measured from a suite of known standards for all elements of interest. The reference element $R$ is usually taken as a matrix element. 
In the analysis of an unknown, the sensitivity factor can be used to convert a measured secondary ion intensity ratio, $i_{x} / i_{R}$, into a concentration ratio:

$$
C_{x} / C_{R}=\frac{1}{S_{x / R}} \frac{I_{x}}{I_{R}}
$$

where $I=i / f$, the total secondary ion signal for all isotopes of an element. By measuring a11 elements in a sample, and assuming

$$
\Sigma C_{X}+C_{R}=1
$$

the absolute value of $C_{R}$ can be determined, and by use of equation (2), all of the $C_{x}$ values can be found, giving a total analysis of the sample. The accuracy of such a procedure depends to a great extent on how strongly the sensitivity factors depend on the matrix. Although absolute sensitivity can vary strongly, relative sensitivity is not as susceptible to matrix effects. This is especially true in the case of highly oxidized samples, in which the sample typically contains 60 atomic percent oxygen. When such a target is analyzed with an oxygen primary ion beam, which further increases the oxygen content, the secondary ions are formed in an oxygen-rich environment, producing a similar matrix despite differences in the electropositive matrix species. The distribution of errors which has been observed in the actual analysis of samples by the method of relative sensitivity factors is shown in figure 2. Approximately 50 percent of the analyses fall within a factor of 1.2 of the correct value, and 80 percent within a factor of 2 .

\section{Physical model}

In principle, it should be possible to predict the secondary ion emission from a multielement target by means of an appropriate model of secondary ion emission, and, consequently, to relate measured secondary ion intensities to the composition. A number of models of secondary ion emission are available. The most successful model for describing secondary ion emission from multi-element oxidic samples is the local thermal equilibrium (LTE) model $(6,8)$. The LTE model describes the region of secondary ion formation as a dense plasma in thermal equilibrium. The ratio of sputtered ions to neutral atoms in this plasma is related to the temperature and electron density, as well as material parameters such as ionization potential and partition functions. In applying the LfE model to a practical analysis situation, the temperature and electron density appropriate to the analysis conditions are derived from the measured secondary ion intensities and known concentrations for two elements in the sample. These values of the temperature and electron density can then be employed to relate the measured secondary ion intensities of the remaining unknown elements to composition. Use of the LTE model thus requires some prior knowledge of the specimen composition, preferably the compositional values of the major elements. In this regard, it is not as flexible in application as the sensitivity factor method.

As an example of the accuracy which can be achieved with the LTE model for oxidic samples, an error factor histogram is shown in figure 3. This histogram has been compiled from analyses of glass standards of known composition, testing various combinations of elements as "internal standards" for analysis. The distribution of errors is such that about 50 percent of the analyses fall within a factor of two of the correct value, and 80 percent within a factor of five.

\section{Detection of Compounds}

In addition to the elemental signals available in the SIMS spectrum, peaks which correspond to molecular constitutents of a sample are frequently observed. For example, in the spectrum shown in figure 1 , a number of $\mathrm{di}$ - and tri-atomic molecules can be observed, including $\mathrm{Fe}_{2}, \mathrm{Cu}_{2}, \mathrm{SiO}, \mathrm{FeO}, \mathrm{FeCa}, \mathrm{Fe}_{2} \mathrm{O}, \mathrm{Fe}_{2} \mathrm{Ca}$. Benninghoven et al. (9) have demonstrated that complicated organic molecules of a molecular weight as high as 300 can be successfully sputtered. In order to observe complicated molecules, it is generally necessary to minimize 


\section{Blind Sample Round Robin}

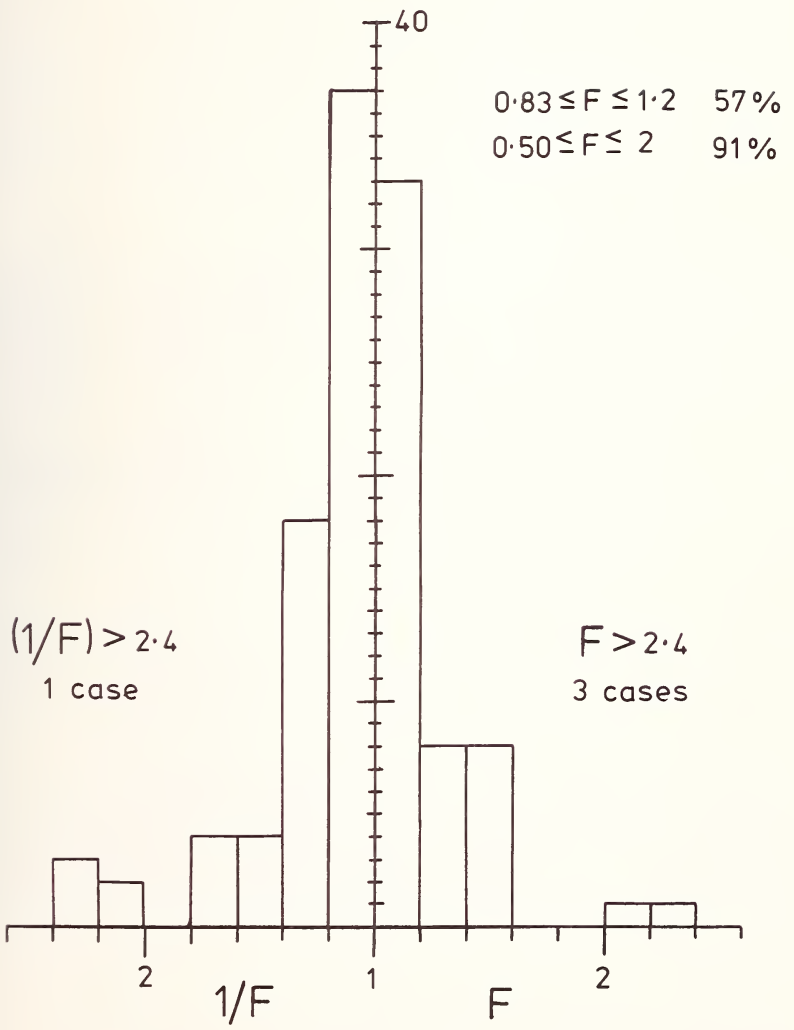

Figure 2. Error histogram of quantitative ion microprobe analyses performed by the method of relative sensitivity factors by four independent laboratories. Specimens: Multi-component silicate glasses. 


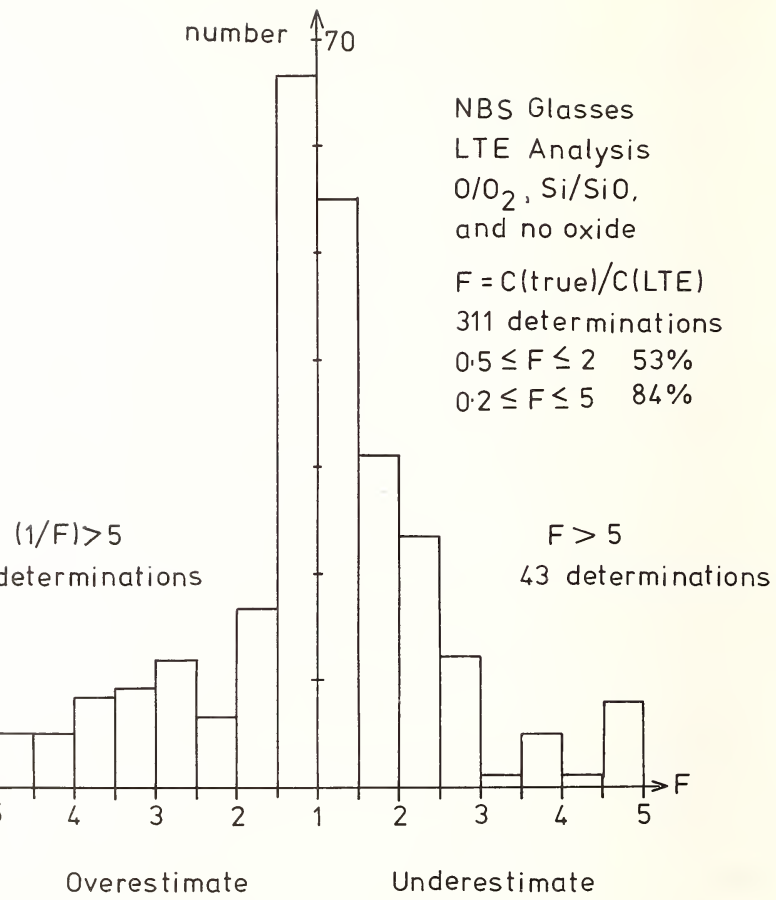

Figure 3. Error histogram of quantitative ion microprobe analyses with data reduction with the local thermal equilibrium model. Specimens: Multi-component silicate glasses.

the damage to the surface caused by prior ion bombardment through the use of low primary ion current densities. Benninghoven identifies conditions of static and dynamic SIMS (10). In static SIMS, the current density, typically less than $10^{-9} \mathrm{~A} / \mathrm{cm}^{2}$, is so low that any ion striking the surface has a very low probability of striking a site disrupted previously by another energetic ion. The total dose during an experiment is generally less than $10^{13} / \mathrm{cm}^{2}$, and considering the number of surface sites as $10^{15} / \mathrm{cm}^{2}$, only about $1 / 100$ of the surface atoms are sputtered. The surface thus remains in an unperturbed or "static" state. In dynamic SIMS, the current density is in the range of $10^{-3} \mathrm{~A} / \mathrm{cm}^{2}$ or more. The ion damage to the surface is so extensive that virtually all surface sites will be hit by ions during recording of the spectrum. The molecules at the surface are thus likely to be broken down during the measurement process, preventing a representative spectrum from being obtained. 
The current densities necessary to achieve a true static SIMS condition are so low that order to obtain an adequate total current for practical measurement, about $10^{-10} \mathrm{~A}$, the am size must be of the order of $1 \mathrm{~mm}$ diameter or greater. In order to apply a static SIMS periment to the study of particles, it would therefore be necessary to examine aggregates many particles. In recent experiments with the ion microprobe operating with a current nsity of $10^{-6} \mathrm{~A} / \mathrm{cm}^{2}$, midway between true static SIMS $\left(10^{-9} \mathrm{~A} / \mathrm{cm}^{2}\right)$ and the dynamic SIMS of e typical high current density ion probe $\left(10^{-1} \mathrm{~A} / \mathrm{cm}^{2}\right)$, we have been able to observe molecu$r$ spectra from a number of compounds, including glycine and fluorodopamine, Figures 4(a) d 4 (b). The principal molecular signals were found to include parent molecule species, agments similar to those patterns seen in the electron impact ionization mass spectrometry, d complexes with the substrate metal atoms. Peak-to-background ratios as high as 1000:1 uld be produced with total beam currents of 1 nA. Spectra were successfully obtained from ese compounds dispersed onto single silver particles as small as $20 \mu \mathrm{m}$ in diameter. ese experiments suggest that, at least for some compounds, SIMS at intermediate current nsities can be used to study organic layers on individual particles, as well as studying eciation from spectra of inorganic compounds.

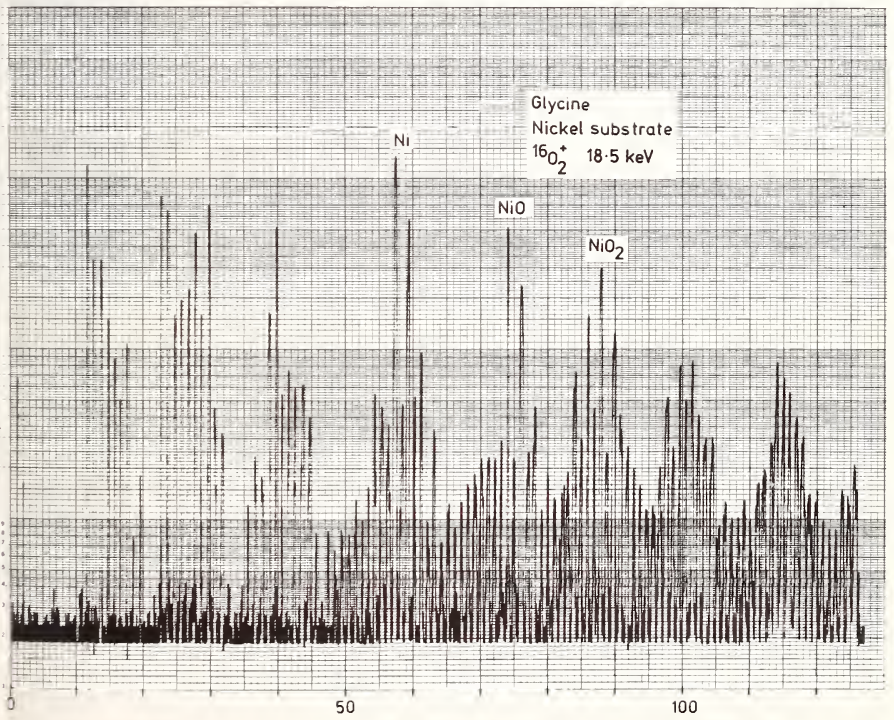

igure 4(a). Positive secondary ion mass spectrum of glycine applied to a nickel substrate. 


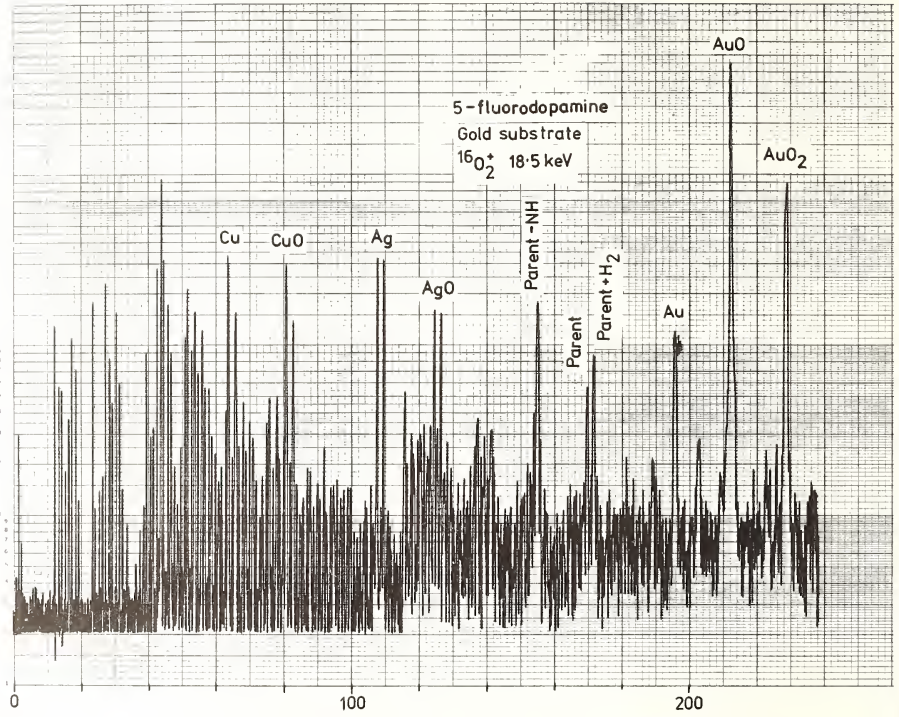

Figure 4(b). Positive secondary ion mass spectrum of 5-fluorodopamine applied to a gold substrate.

\section{Depth Profiling}

One of the most powerful modes of operation in SIMS is that of depth profiling. Since the sample is worn away in a controlled fashion and at any time the secondary ion signal is produced from a surface layer only $1 \mathrm{~nm}$ deep, it is possible to follow the distribution in depth of an elemental or simple compound species. For an optimum sample geometry, flat with no appreciable surface topography, and with controlled sputtering rates, profiles with a depth resolution of $5 \mathrm{~nm}$ are possible (11). The depth resolution is ultimately limited by a number of physical phenomena, including knock-on of sample atoms by the bombarding ions, ion channeling, and the development of topography due to sputtering. The maximum depth attainable is limited to about $5 \mu \mathrm{m}$ due to time and stability considerations.

Depth profiling can be successfully applied to individual particles, although the depth resolution is degraded somewhat by the irregular topography of a particle. An example of a depth profile following several elements in a single urban airborne particulate $10 \mu \mathrm{m}$ in diameter is shown in Figure 5. In order to minimize the effects of variations in the matrix oxygen content upon the secondary ion yields, the signals were normalized with the signal for silicon, a major constituent. Of principal interest is the behavior of the barium and lead constituents, which appear to be concentrated by more than an order of magnitude at the surface of the sample. In addition, a discontinuity is observed, suggesting that the particle is layered. 


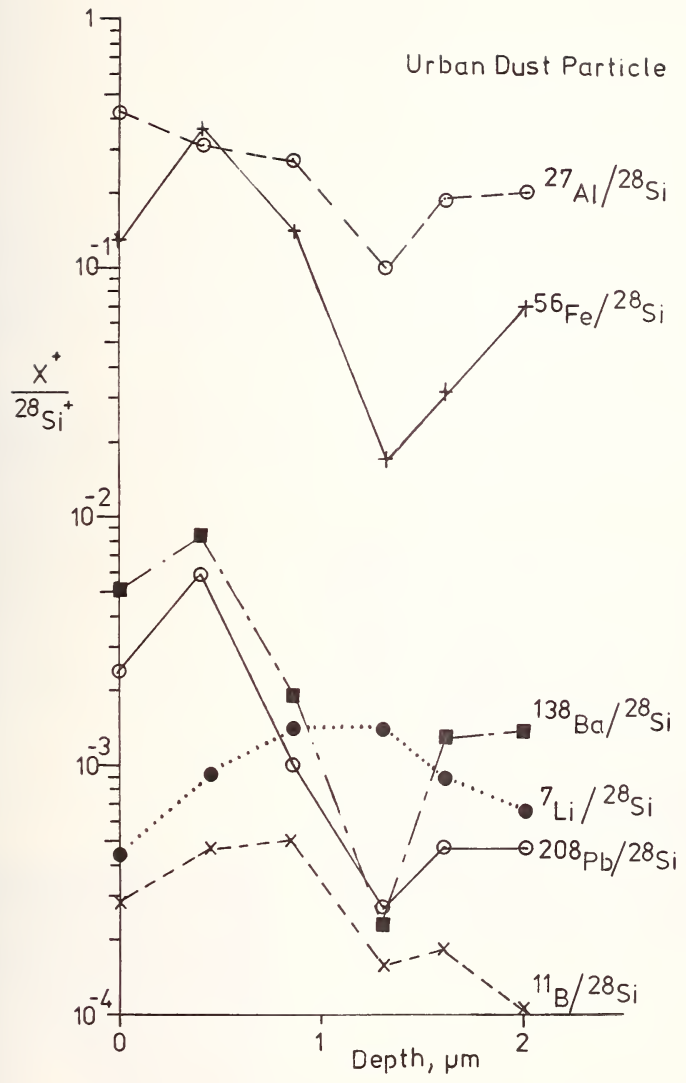

Figure 5. Elemental depth profiles for several elements in a single urban airborne particle with a diameter of $10 \mu \mathrm{m}$. 
In order to make maximum use of the depth profiling mode, it is useful to have a spectrometer which can be rapidly switched from one peak of interest to another to achieve a near simultaneous data collection, or a multi-channel spectrum. Since a typical particle can easily have fifteen or more constituents of interest, the data handling problem can be quite difficult. Since SIMS by necessity must destroy the sample as it is examined, it would be particularly interesting to have truly simultaneous data collection for several ion species, so that the location of the constituents could be more precisely determined.

\section{Ion Imaging}

Ion images showing the spatial distribution of an elemental or molecular constituent can be obtained in the ion microscope or the ion microprobe $(12,13)$. In the ion microscope, a true image is produced by a lens system coupled with a mass spectrometer. The limiting lateral resolution is about $0.5 \mu \mathrm{m}$. In the ion microprobe, the image is constructed by scanning a finely focussed ion beam over a raster and intensity-modulating a cathode ray tube scanned in synchronism with a signal derived from a particular mass peak. In the ion microprobe, the limiting beam size is about $1 \mu \mathrm{m}$, although with a suitable primary ion condenser lens system, a probe size of $0.1 \mu \mathrm{m}$ should be possible.

As an example, figure 6 shows an image with the ${ }^{23} \mathrm{Na}^{+}$signal obtained from a contamination particle on a silicon wafer. An edge resolution of about $1 \mu \mathrm{m}$ is achieved. The scanning time and sputtering rate was such that an average of $2 \mathrm{~nm}$ of material was removed while preparing the image. Besides elemental signals, molecular signals can also be used for the preparation of ion images.

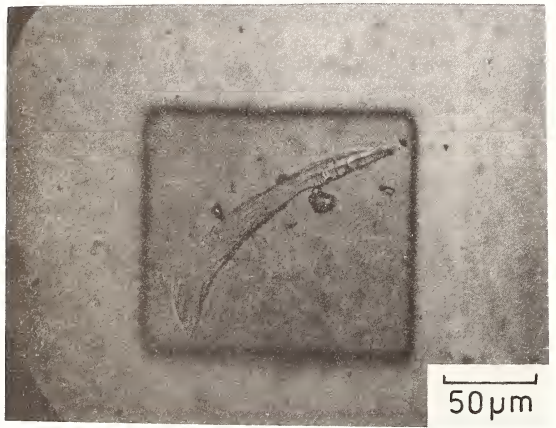

(a)

(b)

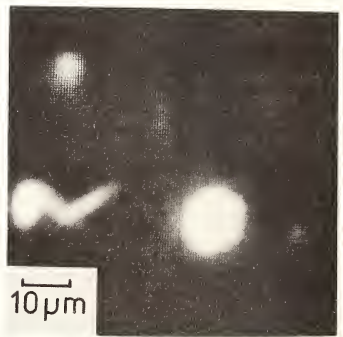

Figure 6. Secondary ion image of a sodium-rich particle on a silicon substrate. (a) Optical image, (b) Scanning ion image. 
Because of the strong variations in the secondary ion signals with different matrix fects, the direct interpretation of image intensities in secondary ion images in terms of incentration is difficult. It is necessary to record the data digitally, apply quantitative irrections by the sensitivity factor or local thermal equilibrium methods, and then reconruct a final compositional image (14).

\section{Summary}

Secondary ion mass spectrometry offers a number of powerful capabilities for the udy of individual particles: (1) the detection of all elements and isotopes; (2) limits detection in the microbeam mode in the range of $1 \mathrm{ppm}$; (3) the possibility of detecting ganic and inorganic compounds; (4) the determination of the distribution in depth of a onstituent, with a high depth resolution; (5) high lateral specificity; (6) the capability preparing ion images showing the lateral distribution of a constituent. Quantitative halysis with a relative accuracy of a factor of 2 is routinely available by the method of elative sensitivity factors or by a physical model for secondary ion emission. The reatest potential of SIMS will be obtained when it is used in conjunction with other urface analysis and microanalysis methods, such as Auger electron spectroscopy, scanning iectron microscopy with $x$-ray microanalysis, and laser Raman microanalysis.

\section{References}

Small, J. A. and Zoller, W. H., "Single Particle Analysis of the Ash from the Dickerson Coal-Fired Power Plant" in Methods and Standards for Environmental Measurement, ed. Kirchhoff, W. H., National Bureau of Standards Special Publication 464 (Washington, 1977).

2] Secondary Ion Mass Spectrometry, eds. Heinrich, K. F. J. and Newbury, D. E., National Bureau of Standards Special Publication 427 (Washington, 1975).

3] McHugh, J. A., "Secondary Ion Mass Spectrometry" in Methods of Surface Analysis, ed. Czanderna, A. W. (Elsevier, Amsterdam, 1975) 223-278.

7] Newbury, D. E., "Secondary Ion Mass Spectrometry for Particulate Analysis" in Environmental Pollutants Detection and Measurement, eds. Toribara, T. Y., Coleman, J. R., Dahneke, B. E., and Feidman, I. (Plenum, 1978) 317-348.

5] Czanderna, A. W., Methods of Surface Analysis (Elsevier, Amsterdam, 1975).

6] Andersen, C. A., "Analytic Methods and Applications of the Ion.Microprobe Mass Analyzer" in Microprobe Analysis, ed. Andersen, C. A. (Wiley, 1973) 531-553.

7] Newbury, D. E., "Quantitative Analysis by Secondary Ion Mass Spectrometry" in Quantitative Surface Analys is of Materials, ed. McIntyre, N. S., ASTM STP 643 (ASTM, Philadephia, 1978) 127-149.

8] Andersen, C. A. and Hinthorne, J. R., "Thermodynamic Approach to the Quantitative Interpretation of Sputtered Ion Mass Spectra", Analyt. Chem., 45 (1973) 1422-1438.

9] Benninghoven, A., Jaspers, D., and Sichtermann, W., "Secondary Ion Emission of Amino Acids" Appl. Phys. 11 (1976) 35-39.

10] Benninghoven, A., Surface Sci. 53 (1975) 596.

11] McHugh, J. A., "Factors that Influence an Elemental Depth Profile", in NBS SP 427, ibid, 179-189.

12] Morrison, G. H. and Slodzian, G., "Ion Microscopy", Analyt. Chem. 47 (1975) 932A-943A.

13] Newbury, D. E., "Ion Imaging in Secondary Ion Mass Spectrometry", in Multidisciplinary Microscopy, Proc. Soc. Photo-Optical Instrumentation Engrs., 104 (1977) 85-89.

14] Morrison, G. H., "Digital Image Processing in Ion Microscopy," Proc. 2nd U. S. Japan Seminar on Secondary Ion Mass Spectrometry", Osaka (Oct. 23-27, 1978). 
[13] Newbury, D. E., "Ion Imaging in Secondary Ion Mass Spectrometry", in Multidisciplinary Microscopy, Proc. Soc. Photo-Optical Instrumentation Engrs., 104 (1977) 85-89.

[14] Morrison, G. H., "Digital Image Processing in Ion Microscopy," Proc. 2nd U. S. Japan Seminar on Secondary Ion Mass Spectrometry", Osaka (0ct. 23-27, 1978). 
National Bureau of Standards Special Publication 533. This Publication Originated from a ipecial Session on Particle Analysis, 13th Annual Conference of the Microbeam Analysis jociety, Ann Arbor, MI, June 22, 1978. (Issued April 1980)

\title{
SCOPE AND LIMITATIONS OF SINGLE PARTICLE ANALYSIS BY RAMAN MICROPROBE SPECTROSCOPY
}

\author{
Edgar S. Etz and John J. Blaha \\ Center for Analytical Chemistry \\ National Bureau of Standards \\ Washington, D. C. 20234
}

\section{Introduction}

From the time of its discovery in 1928 until a few years ago, Raman scattering was used is a tool for the study of bulk samples of macroscopic dimensions, furnishing information about fundamental molecular properties as well as serving an important function in laboratory spectrochemical analysis $[1-3]^{1}$. The advent of lasers as sources for excitation of Raman ipectra and the ongoing developments in instrumentation for optical spectroscopy have increased the sensitivity of Raman scattering experiments to the point where vibrational spectra can now routinely be obtained from discrete microsamples or sampling volumes of nass approaching one picogram [4-7]. Raman measurements in the microscopic domain are peing conducted in a number of laboratories throughout the world and at present involve the use of two types of instruments. One is the Raman microprobe developed at NBS [6] specifically for the analysis of micrometer-size particles, and the other is the now commercially available Raman microprobe/microscope MOLE [7].

The fundamentals of Raman microprobe spectroscopy and the microanalytical applications (covering the literature through 1978) of these new instruments have recently been reviewed [8]. Detail on critical aspects of instrument design and performance and on measurement criteria important to optimized microanalysis has been reported elsewhere $[9,10]$.

The emphasis of the work published to date has been to demonstrate the practical uses of the technique in several major application areas, and a few of these investigations can se cited here as being illustrative of this research [7-18]. The problems which have been examined fall into the broad areas of (i) materials analysis and characterization of microstructure $[7,11,12]$, (ii) particulate contamination studies $[7,11]$, (iii) environmental varticulate pollution analysis [13-15], (iv) micromineralogy and mineral inclusion studies $[9,12,16]$, and $(v)$ investigations of biological and pathological tissues $[, 17,18]$.

Much has been learned in the course of these studies concerning the principal advantages and inherent shortcomings of Raman microprobe analysis $[8,10]$. The recrgnition of these aspects permits projections to be made as to the further development nd future jotential of Raman scattering as a viable probe technique [19-23].

In this paper we will present our current views of the scope and limitations of Raman nicroprobe spectroscopy. This perspective is largely based on our experiences resulting from the development at NBS of micro-Raman instrumentation and the application of the technique to a diversity of microanalytical problems. We will briefly review the fundamentals of laser-Raman spectroscopy and show how, with present methodology, the principles of normal (or spontaneous) Raman scattering have successfully been applied to chemical nicroanalysis. This will include a description of the design and performance of the NBS developed microprobe and comments on the MOLE (Molecular Optical Laser Examiner) microprobe simultaneously developed in France. The general analytical characteristics of these microprobes are demonstrated by measurement results obtained with the NBS instrument. These are addressed in terms of the basic parameters of interest, including spatial resolution, sampling volume, limits of detection, and spectral specificity. Examples of application will emphasize results from the analysis of single particles, supplemented by selected illustrations of capability for the investigation of the other types of microsamples. The

${ }^{1}$ Figures in brackets indicate the literature references at the end of this paper. 
experimental limitations of the technique are discussed in relation to laser-induced sample heating and fluorescence, the chemical reactivity of microparticles, the intrinsic weakness of the Raman effect, and the lack of an adequately developed theory for measurements to be placed on a quantitative basis. The results obtained in the areas identified above demonstrate the broad applicability of this new method of microanalysis and illustrate the unique information that can be obtained from micro-Raman scattering experiments. These results also suggest numerous other opportunities for significant research in materials characterization and, we believe, are certain to contribute to the further development of the technique.

\section{Fundamentals of Raman Microprobe Spectroscopy}

\section{A. The Analytical Goal}

The most widely used techniques of instrumental microanalysis readily identify and determine the quantity of elemental constituents present but do not distinguish the chemical forms of elements present as specific compounds in a microsample. The few physical methods of microanalysis that permit obtaining information about the compounds present include techniques such as $x$-ray photoelectron spectroscopy (XPS or ESCA), x-ray diffraction (XRD), infrared spectroscopy (IR), and thermoanalysis. These provide possibilities for the qualitative identification and, in some cases, even the quantitative determination of molecular species - such as $C, N, S$ compounds - and are coming into more widespread use for the total characterization of environmental particulate matter [24]. None of these compoundspecific techniques, however, are truly microprobe methods in that the analytical signal is obtained from a single particle a few $(<10 \mu \mathrm{m})$ micrometers in size or from a microscopic sampling volume of equivalent dimensions. By these methods, the analytical information is generally obtained from a considerably larger sample mass (typically in the microgram to milligram range) which in the case of dust analysis requires a large number of particles.

Micro-Raman spectroscopy now provides a powerful complement to these techniques by enabling routine, compound-specific microanalysis of discrete microscopic particles or probe regions approaching 1 picogram in sample mass [8]. Among the chief advantages of the method are that it is non-destructive of the sample, information on chemical species is obtained regardless of the physical state (usually solid or liquid) of the sample, and with respect to solids - the Raman spectra are analytically diagnostic for both crystalline and amorphous phases. Raman spectroscopy, therefore, can be considered a highly specific and sensitive technique of investigating molecular vibrational phenomena in all phases of matter. Applied to chemical microanalysis, it is an unequaled tool for the characterization and identification of principal molecular constituents which are fingerprinted by their vibrational spectra. In addition, as was inferred above, these spectra most often contain information on the local molecular environment and the structural order of the solid phase (crystalline or glassy state) in which the species resides.

\section{B. The Raman Scattering Process}

The spectroscopic measurements performed with today's Raman microprobes are based upon the excitation of the normal or spontaneous Raman effect. This effect provides the basis for conventional Raman spectroscopy and has been extensively reviewed in the context of modern chemical analysis $[1-3,25]$. The measurement consists of the study of the spectral distribution of inelastically scattered light emitted by the sample irradiated with a monoenergetic, visible beam of laser light. The scattered radiation contains weak lines, at frequencies both lower and higher than the exciting radiation. The frequency differences, called Raman shifts, are characteristic of molecular vibrations in the sample and are independent of the exciting frequency. Thus, these spectra (distributions of scattered light intensity with frequency or wavelength) of the Raman scattered light identify the scattering molecules and - in the case of solids - contain information from which the nature and extent of crystallinity may be inferred. The spectra that are obtained in these instruments are the so-called Stokes-Raman spectra. They arise from molecules which scatter photons of lower frequency (Stokes lines) than that of the exciting line. The corresponding higher frequency (anti-Stokes) lines are, under ordinary conditions, of considerably lower intensity - thus more difficult to detect - and are generally recorded 
7y when special information (e.g., temperature data from Stokes/anti-Stokes intensity tios) on the sample is sought.

Raman scattering is a secondary scattering phenomenon (Mie or elastic scattering is ders of magnitude more intense) and thus Raman line intensities are weak. This places fficult requirements on the performance of a Raman spectrometer designed for microalytical applications. In a typical Raman experiment - for scattering by solids - only e photon from the $10^{6}-10^{8}$ incident on the sample is scattered inelastically by molecular brations. For this reason, intense excitation sources, perfect optics for collecting e scattered radiation, and a highly efficient monochromator coupled to a low-background otodetector are required to successfully detect a Raman 1 ine.

The intensity of the Raman scattered light is proportional to the fourth power of the equency of the incident radiation. Band intensities are determined by the cross section $r$ Raman scattering and are directly proportional to the number density or concentration scattering molecules in a specific energy state. Because of the indicated frequency pendence of the Raman signal and the lack of highly effective detectors in the red and ar infrared range, the frequency of the exciting radiation must be in the visible, or tter, toward the ultraviolet range. In the case of excitation in the near ultraviolet, serious difficulty may arise caused by the fluorescence of molecules in the Stokes range.

\section{The Raman Information Content}

Raman spectroscopy can be used for the analysis of polyatomic molecules or ions whose larizability changes during vibration (the requirement for infrared activity is a change the dipole moment). Most materials - sulfates, nitrates, carbonates, oxides, silicates, monium salts, and organic compounds - show characteristic Raman-active vibrations nternal modes). Structural information is derived via the symmetry of molecules, the imber and strength of chemical bonds, and the atomic masses which determine the position

Raman bands. This allows distinguishing between compounds having the same elemental mposition but different molecular coordination and structure.

A further degree of specificity of the Raman spectrum - in the case of solids - derives om motions of the crystal lattice as a whole that give rise to external or lattice vibraons. These modes are usually seen in the low-frequency region (below $400 \mathrm{~cm}^{-1}$, Raman ift) and generally do not couple with the structure-specific internal modes.

Important to microanalysis is the experimental observation that the Raman frequencies molecules present in microsamples (e.g., microparticles) are no different from those served in the spectra of macroscopic samples. Thus the selection rules for Raman :attering appear to be preserved. This correspondence among the spectra of particles and ieir bulk sample counterparts permits the use of Raman data reported in the literature as source of reference for the identification of chemical species in unknown particles.

One other point is noteworthy in the application of the technique to micro-analysis. is is the fact that even though the discriminating power of the Raman effect may not ways remove spectral ambiguities due to interfering molecules, the microsample is less kely to consist of a broad mixture of substances than the bulk of the material or larger amples from it. Consequently, the likelihood of several major, spectroscopically interering components in the microsample is usually small. This can be expected to facilitate le interpretation of micro-Raman spectra.

\section{Experimental Methods and Techniques}

\section{Requirements for Microsample Analysis}

The various criteria important to Raman analysis of microsamples, and microparticles 1 particular, have been described $[5,6,9,10]$. These center on a number of specific istrument performance requirements which must be met by a practical Raman microprobe as 211 as on the judicious choice of several measurement parameters to ensure non-destructive alysis. In the former category is the requirement for detection of extremely low signal evels. This includes the need for full optimization of the signal from the sample 
(frequently of mass $\sim 10^{-12} \mathrm{~g}$, as in the case of a single microparticle) and the effective rejection of optical interferences. Among the various sources of optical interference are the high light levels from the intense elastic scattering of the incident beam by the sample and the supporting substrate (or the host) and the spectral interferences from the Raman scattering by the substrate (or the host) itself. Crucial also is the level of irradiance (or power density, in watts per square centimeter) directed at the sample in a focused beam a few micrometers in diameter (cross section). A third consideration, related to the first, is the choice of substrate material used for supporting samples for measurement.

The irradiance levels typically required to permit the observation of the spectrum from a small particle are apt to cause significant sample heating in cases where the particle absorbs some or all of the energy at the exciting frequency. For this reason, any radiant energy absorbed by the particle and converted into heat must rapidly be dissipated. An important function of the substrate is therefore to provide an effective heat sink, thereby maintaining the temperature of the sample to values permitting non-destructive analysis. The selection of the irradiance level and substrate material is particularly pertinent in the analysis of environmental particles for which the optical absorption properties are not known or easily determined.

In the following sections we describe the basic instrumentation required for microRaman scattering experiments, present a discussion of the important design/performance features of the NBS microprobe and give an overview of the characteristics and measurement capabilities of the MOLE Raman microprobe/microscope. This is followed by some experimental detail on sample preparation and measurement procedures as applied to particles with a discussion of experimental limitations of the Raman microprobe measurement.

\section{B. Instrumentation for Micro-Raman Spectroscopy}

\section{The Basic Raman Experiment}

We will give a brief survey here of the basic instrumentation available for micro-Raman scattering experiments. Only those components of the general instrument configuration are considered which - by design and performance - are important to microanalytical applications. In this context it is useful to discuss micro-Raman instrumentation under the headings of (i) excitation sources, ( $i i)$ monochromators and spectrographs, ( $i i i)$ detectors and recording systems, (iv) sampling arrangements, and (v) computer techniques. A thorough discussion of instrumentation for conventional, macro-Raman spectroscopy can be found in the literature [26]. The analytical applications of lasers in the field of atomic and molecular spectroscopy have recently been presented in a broad coverage of the topic [27].

(a) Excitation Sources. In microprobes now in use, gas lasers are used as excitation sources. These lasers provide concentrated, polarized, coherent, and highly monochromatic radiation. The intensity of the exciting line and the frequency of excitation may be varied. The laser lines most commonly used lie in the visible region of the spectrum, and good continuous power levels (output power of up to $200 \mathrm{~mW}$ in a given 1 ine is adequate for most micro-Raman work) are readily attainable from lasers that are commercially available. The gas lasers in most common use are the argon ion $\left(\mathrm{Ar}^{+}\right)$, krypton ion $\left(\mathrm{Kr}^{+}\right)$, and mixed gas $\left(\mathrm{Ar}^{+} / \mathrm{Kr}^{+}\right)$lasers which conveniently provide strong output lines from the blue to the red at principal wavelengths $488.0 \mathrm{~nm}$ (blue), $514.5 \mathrm{~nm}$ (green), $568.2 \mathrm{~nm}$ (yellow), and $647.1 \mathrm{~nm}$ (red). A helium-neon laser ( $\mathrm{He} / \mathrm{Ne}$ ) may also be used. Its primary output wavelength is at $638.2 \mathrm{~nm}$.

In addition to gas lasers, continuous wave (cw) dye lasers that can be tuned to any frequency from yellow to red with power outputs of several hundred milliwatts are increasingly being used in Raman spectroscopy. However, these have not been employed so far in micro-Raman investigations. They suffer from the problem that the dye laser emission has a relatively high background.

The choice of the exciting line is dictated by the color of the sample. The requirement that the sample be transparent (ideally) to the incident radiation means that for spectral excitation a line should be chosen that most closely matches the color of the sample. For colorless (transparent) samples any exciting line in the visible region may be used. 
(b) Monochromators and Spectrographs. The (scanning) monochromator of the Raman nstrument separates the very weak Raman signal from the considerably more intense Rayleigh i.e., elastic component) scattered and reflected radiation. In addition to the desirable haracteristics of good resolution and low stray light levels, the monochromator should have high light throughput (i.e., light gathering efficiency), since the limit of measurement ensitivity is invariably governed by the intensity of the scattered light reaching the etector. All of these considerations have been approached in different ways by various esigns. The most advanced spectrometer systems now in use employ double or triple monohromators fitted with new types of gratings. The recently developed holographic gratings hich are now commonly employed, exhibit superb stray light capability. For example, the oncave holographic grating double monochromators used in Raman microprobes have a stated tray 1 ight rejection of $10^{-12}$ at $20 \mathrm{~cm}^{-1}$ (frequency shift from the exciting 1 ine). 0 ther ttributes of these gratings are high dispersion, good spectral resolution, and the spectra re completely free from grating ghosts (i.e., spurious lines).

New types of spectrographs have been developed in recent years that take advantage of he stray light rejection capabilities of holographic gratings. In micro-Raman instrumentaion, these spectrographs are employed with multichannel spectral analyzers using imagentensified TV-type detectors (e.g., vidicon tubes) [7,22]. In these low light level pplications, the photosensitive surface of the detector is placed in the focal plane of the arge-aperture spectrograph. In this way, either a major segment or the entire spectrum is ocused on the detector active area from where it can be recorded onto a read-out device. - mechanical scanning is necessary with this technique which permits the recording of pectra in fractions of a second (e.g., $\sim 10^{-3} \mathrm{sec}$, in principle), thus making the observaion of transient species possible (as in kinetic studies).

(c) Detectors and Recording Systems. (i) Photomultipliers. Despite recent advances n spectroscopic detection systems, photomultipliers (PMT) are still the detectors which rovide lowest noise for very low light level signals in the range from the ultraviolet to bout $850 \mathrm{~nm}$. The primary advantage of photomultipliers is their amplification, by factors in the order of $10^{6}$, with very low noise. The sensitivity (or spectral response) of these levices when used in the photon counting mode is determined by their quantum efficiencies, and these are a function of the wavelength region in which they are employed. Typical juantum efficiencies for a tube with $\mathrm{S}-20$ response photocathode are 25 percent at $400 \mathrm{~nm}$, 3 percent at $700 \mathrm{~nm}$, and 0.3 percent at $800 \mathrm{~nm}$. For this reason, excitation of the sample lith the blue or green 1 ine from an $\mathrm{Ar}^{+}$laser is preferred to that with the red lines from a le/Ne or $\mathrm{Kr}^{+} \mathrm{laser}$, provided that sample fluorescence or sample heating (due to radiation absorption) is not a limitation.

For the kinds of applications considered here the photomultiplier tube is usually cooled down to $-30{ }^{\circ} \mathrm{C}$ to reduce the dark count to values less than five counts per second. in many measurement situations where the background count is negligible, cooling therefore urovides an easy way to increase the signal-to-noise $(\mathrm{S} / \mathrm{N})$ ratio. Typical signal detection ates in Raman microprobe measurements of micrometer-sized particles may vary from a low of $20-30$ counts/sec to signal rates frequently exceeding $10^{4}$ counts $/ \mathrm{sec}$.

(ii) Spectral Multichannel Detectors. A second category of spectroscopic detectors is developing at an accelerated rate and these are the optical multichannel detectors. These detection systems offer an important advantage over a conventional photomultiplier urincipally from the point of view of data handling capability. These detectors allow data acquisition times to be significantly reduced - compared with conventional single channel pperation - in proportion to the number of wavelength resolved intervals that are simultaleously detected by the device. They consist of image-intensified, TV-type detectors (or zameras) composed of a combination of one or more optical image-intensifiers and a twodimensional transducer such as a vidicon or diode array. This type of multichannel detector zan be used to provide sufficient amplification for photon counting sensitivity over a twodimensional matrix. This combination is particularly useful for Raman microprobe measurenents because it permits simultaneous spatial and spectral multiplexing. The principles underlying these multichannel detectors (or image devices) and their uses in analytical spectroscopy have recently been reviewed [28-30]. A number of these optical multichannel devices are now available as complete detector systems and are commonly known as silicon vidicon, silicon intensified target vidicon (SIT), intensified SIT, and intensified diode array (IDA) detectors. The performance of these devices vs. photomultiplier detectors in optical spectroscopy has been described with particular emphasis on signal-to-noise 
relationships [31]. The most recent versions of these systems are becoming available as cooled detectors and possess improved analytical potential with respect to photon counting sensitivity, dynamic range, signal-to-noise ratio and data handling capability $[20,32]$.

Research efforts are underway to fully explore the combined capabilities of advanced, novel-design holographic grating spectrometer/spectrographs and various forms of these optical multichannel analyzer (OMA) systems to yield Raman microprobe instrumentation with sensitivities truly approaching those of photomultipliers, but having the multiplex advantage with high resolution [22]. The presently available commercial versions of these 0MAs are computer-compatible and provide expanded capabilities for data presentation and manipulation. These systems wiil further facilitate Raman scattering studies from microsamples and should enhance the possibilities for non-destructive analysis, because measurement times can be significantly reduced.

(iii) Recording Systems. Traditionally, the output of the photomultiplier detector is recorded in one of two ways. The least expensive method, d.c. amplification, works well at both high and low light levels, and the analog signal is recorded on a strip chart recorder. Clearly, the method of choice in micro-Raman spectroscopy is signal detection by photon counting which offers greater versatility as the digital output is directly compatible with other digital equipment such as signal averagers and computers.

(d) Sampling Arrangements. The most important criterion in the sampling arrangement of a Raman microprobe is the illumination/collection geometry utilized for the generation and efficient gathering of the scattered radiation. The foremost aim is to provide efficient illumination and collection and to select a design that will minimize optical interferences.

The most common scattering geometries in Raman spectroscopy employ either $90^{\circ}$ or $180^{\circ}$ (angle of laser beam incident on sample, relative to direction of collection) illumination. The two types of Raman microprobes now in use both employ a backscattering geometry. The particular designs utilized in both instruments - and the advantages derived from them - have been described $[6,7,23]$ and will only briefly be reviewed in subsequent discussions.

In choosing an appropriate scattering configuration to obtain spectra from microsamples, factors to be considered include maximizing the collected Raman signal, minimizing the amount of elastically scattered light which enters the spectrometer, and minimizing any spectral contributions from the supporting substrate. The backscattering geometry is an effective means for the collection of scattered light and minimizes scattering of stray laser light into the spectrometer. It is also of particular advantage for the probing of strongly absorbing materials, as the focused laser penetrates only little (by perhaps only a few tenths of a micrometer) into the sample surface making a backscattering configuration necessary. Beyond the collection optics, a means must be provided for efficient optical coupling between the sample and the monochromator. This is achieved by appropriate collection lenses matched to the optical aperture of the spectrometer.

One other factor, mentioned earlier, is the presence of the supporting substrate (or the host) which may lead to spectral interferences in the Raman scattering from microsamples. In this regard the substrate material itself should be non-interfering, i.e., have little or no Raman scattering, absorption or fluorescence. In the case of a heterogeneous sample presenting an arbitrary host (e.g., as in the analys is of microparticulate inclusions in a mineral host or in a biological matrix) this requirement is usually not met. In such cases the optical system needs to be optimized so that collection of the light scattered by the microscopic inclusion is maximized and collection of any radiation scattered by the host is minimized.

(e) Computer Techniques. The application of computers in Raman spectroscopy for experiment control, data acquisition, and reduction has led to improvements in data accuracy and to conveniences in data handling. Digital methods and automation techniques for Raman experiments have been described [33-35].

Advances in hardware and software development for spectroscopic applications have made Raman systems enormously powerful. Usually, the functions of controlling the experiment, acquisition and storage of the data, are performed under active computer control, i.e., 
-17ine, while the data evaluation or reduction step is performed of $f$-line at a later time. $t$ is probably in the area of data evaluation and analysis that the computer has had the reatest impact on Raman spectroscopy, providing a powerful method for data manipulations hich were impossible to perform without digital techniques. Fast data display, implementing nstrumental corrections, obtaining difference spectra (addition or subtraction), smoothing f spectra, curve resolution, and peak area determination for calculating the concentration f species, are now commonly performed by computer techniques.

The micro-Raman systems presently in use and those under development are computerompatible and are readily interfaced to either dedicated or time-shared computers to ncrease their capabilities. In these systems, the computer controls the functions of the pectrometer (i.e., the scanning of the wavelength drive, slit settings, etc.), the moveent of the sample stage, and the complete data acquisition process (e.g., digitized photon ounting). Appropriate software designs allow for data refinement (e.g., spectrum stripping", ratioing, etc.), graphic display, and development of a spectral library.

\section{The Raman Microprobe Developed at NBS}

For a detailed description of the design and construction of the NBS Raman microprobe, he reader is referred to accounts given in earlier published work $[6,36]$. The basic layout if the instrument is shown in the schematic of figure 1 and is identical, in principle, to hat of a conventional, monochannel Raman spectrometer. It employs a mixed gas, $\mathrm{Ar}^{+} / \mathrm{Kr}^{-}$ aser for excitation, and any one of the several prominent emission lines are used. Beamlirecting, prefiltering and focusing optics are used to furnish a tightly-focused excitation eam (fore-optics). A separate optical system is utilized for the collection and transfer if the scattered radiation to a state-of-the-art double monochromator equipped with concave

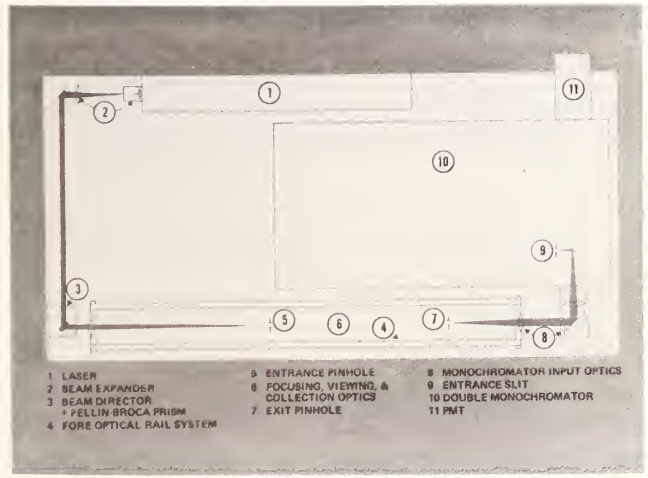

Figure 1. Configuration of the laser-Raman microprobe developed at NBS. The excitation source is a continuous-wave gas - laser. - The fore-optical system (cf. fig. 2) utilizes a backscattering illumination/collection geometry. Spectral analysis is by a scanning monochromator and photomultiplier detection. The instrument is set up on a vibration-isolated optical table (granite). 
holographic gratings (Ramanor HG-2, Instruments SA, Inc., J-Y Optical Systems Division). ${ }^{2}$ A PMT based photon counting electronic detection system and a minicomputer are employed for data acquisition and total system control (e.g., sample stage positioning, wavelength scan, slit settings, etc.). Commercially available optical and mechanical components have been incorporated in the design wherever feasible. Optimum performance is required from each subsystem of the entire spectrometer. High-precision adjustments are provided for each optical and mechanical component. The components utilized to focus the radiation on the sample - most often a single microparticle - position the sample substrate, and collect the scattered radiation are mounted along the optic axis at the center of a four-rail support system. These components are shown in a schematic view in figure 2. A lens (not shown) on the rail focuses the incident laser beam (entering from the left) through the entrance pinhole along the optical axis of the fore-optical system. The entrance pinhole is situated at the image point of a beam-focusing microscope objective. Either a reflecting objective (as shown) or a refractive objective lens are used to provide beam spots that may be varied

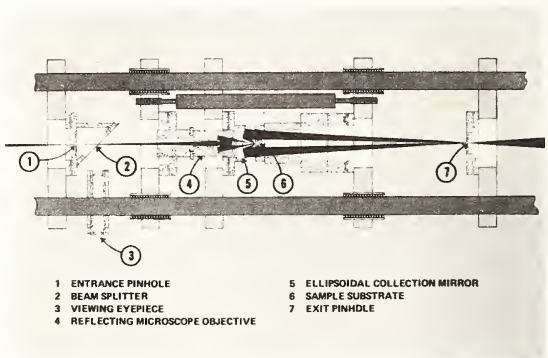

Figure 2. Schematic of the fore-optical system of the NBS Raman microprobe, showing beam focusing, sample viewing, and scattered light collection optics mounted on support rails.

from $\sim 2-20 \mu \mathrm{m}$ in diameter (1ateral resolution). Either of these objectives (with long working distance) focuses the laser beam to a small spot on the sample through a circular opening in the center of an ellipsoidal mirror collector of high numerical aperture (N.A. $0.86)$, thereby providing efficient, large solid-angle collection of the scattered light. The sample, i.e., the particle, held on the surface of a substrate (typically an optically transparent disc of sapphire or lithium fluoride) is mounted perpendicular to the incident beam on a sample stage that can be accurately and reproducibly positioned by remotelycontrolled piezoelectric translators. The beam-focusing objective also serves to view the

\footnotetext{
${ }^{2}$ Certain commercial equipment, instruments, or materials are identified in this paper in order to adequately describe the subject discussed. In no case does such identification imply recommendation or endorsement by the National Bureau of Standards, nor does it imply that the item identified is necessarily the best available for the purpose.
} 
lample, by means of a beam splitter and a viewing eyepiece. In this spectrometer microscope, che sample can be viewed in transmitted light - with a fiber-optic guide mounted behind the substrate - at variable magnifications up to $400 \mathrm{X}$ to aid in the location and precise posicioning of particles of interest. The focal point of the laser beam is made to precisely coincide with the near focus of the ellipsoidal mirror. Exact positioning of the particle at this point then results in the focusing of the collected, scattered light at the second or far focal point of the ellipsoidal mirror which is made the position of an exit pinhole. This pinhole serves to define and limit the effective light collection volume. The collection of radiation scattered by the particle is thereby optimized and (interfering) scattered radiation from the supporting substrate is minimized. The exit pinhole is imaged on the entrance slit of the monochromator. The scattered light at the position of the exit slit is detected by a (thermoelectrically) cooled photomultiplier tube with measured dark zount of under five counts per second. Conventional photon counting equipment is used for amplification and monitoring of the Raman signal.

\section{The Raman Microprobe/Microscope (MOLE) Developed at C.N.R.S.}

The Raman microprobe/microscope (MOLE) that is now commercially available (Instruments SA, Inc., J-Y Optical Systems Division) is based on instrumentation developed several years ago at the Centre National de 1a Recherche Scientifique (C.N.R.S., Lille-France) to obtain Raman spectra of microsamples and images of microscopic features using a characteristic Raman frequency $[4,7]$. Applications of the instrument to chemical/biological microanalysis and to microstructural investigations of solid state materials have been described $[11,12]$. Since the MOLE is the microprobe now being adopted by several laboratories, and instruments of its type are likely to find increasing use, we present here a description of the MOLE that identifies its main features and outlines its analytical function.

\section{Basic Configuration}

The basic configuration of the MOLE results from the coupling of a conventional light microscope (with bright and dark field jllumination) to a scanning spectrometer/spectrograph equipped with concave holographic gratings, followed by a multichannel and/or monochannel detection system. A laser (typically argon ion) is the monochromatic source of light used for irradiating the sample. The light microscope provides the sample stage and forms part of the imaging system. All modes of observation available in a standard optical microscope are available in the instrument. Therefore, microsamples can be examined in situ without any more sample preparation than that required for incident light observation.

The double monochromator spectrometer has very low stray light level and permits the spectral separation (at good resolution) of characteristic Raman lines as they appear in the vibrational spectrum. It is also capable of transmitting spectral line images over a wide spectral range. The associated detection system consists of two separate assemblies, a conventional monochannel detector (e.g., photomultiplier, PM) and a sensitive multichannel optical detection system (typically an intensified vidicon detector). The latter system is linked to a visualization system that displays the micrographic images formed in a spectral line on a CRT. A computer with disc or tape memory can be interfaced to the instrument for data processing and storage. A schematic diagram of the instrument layout is given in figure 3 .

This configuration results in a versatile microprobe/microscope because it permits two fundamentally different and independent modes of operation. One function is that of a molecular microprobe, the other that of a molecular microscope. They can be described as follows (cf. fig. 3 ). 


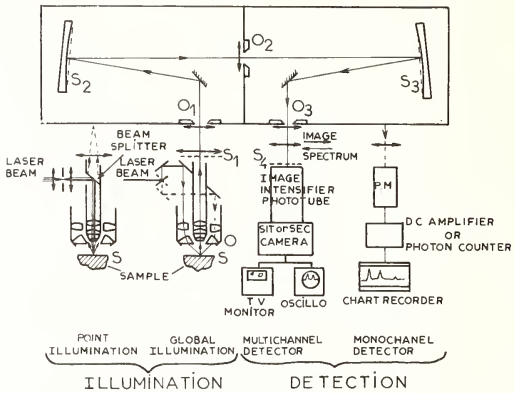

Figure 3. Diagram of the Raman microprobe/microscope (MOLE) developed at C.N.R.S. (Lille-France), illustrating schemes of sample illumination and signal detection. The double grating filter is both a scanning monochromator and a spectrograph allowing two modes of instrument operation (see text).

\section{Modes of Operation}

(a) Point Illumination with Monochannel or Multichannel Detection (Spectral Mode). By use of the bright field illumination system of the microscope, the objective focuses the laser beam into a microscopic spot, and the sample or sample region of interest is moved by translation of the microscope stage into the focal volume of the beam. The light scattered by the sample is collected in the backscattering direction through the same focusing objective and directed into the monochromator/spectrograph (double concave holographic filter). To obtain the Raman spectrum, the optical filter can work as (i) a Raman spectrometer when the detector is a photomultiplier followed by an amplifier and a chart recorder, or (ii) a Raman spectrograph when the detector is an image intensifier tube coupled to a low light level TV camera (SIT or SEC tube).

(b) Area Illumination and Raman Imaging System (Imaging Mode). In this mode, a larger area (typically a circular area, anywhere from $150-400 \mu \mathrm{m}$ in diameter) of the sample is illuminated from above with defocused laser radiation. In this mode of area (or global) illumination, the laser beam is rotated to form an annular beam of light which is directed to the sample by the condenser of a dark field illuminator. By selecting a frequency in the Raman spectrum characteristic of one specific component in the sample, a micrographic image is produced which indicates the distribution of this component throughout the $i l l u m i-$ nated area. This Raman line imaging is achieved by transferring the optical image formed in the microscope onto the gratings, and from there the Raman image is transferred to the photocathode of the intensifier tube/multichannel detector.

In the monochannel spectral mode, classical Raman spectra can be recorded of the entire microsample or a specific volume element thereof. This mode corresponds to the function of the NBS microprobe. In the multichannel spectral mode, the Raman spectra are observed in spectrographic form on an oscilloscope, in real time, where up to $100 \mathrm{~cm}^{-1}$ segments of a spectrum can be displayed. This mode is potentially useful for monitoring time-dependent 
hanges in a sample (e.g., spectral changes due to heating, photo-degradation, etc.). The maging mode allows the localization of individual components of a multicomponent heteroeneous sample (i.e., "mapping" of the distribution of a species) with a resolving power imited, in principle, only by the wavelength of the detected scattered light. In practice, he spatial resolution of the Raman line images is about $1 \mu \mathrm{m}$.

\section{Samples and their Measurement}

\section{Sample Preparation}

Full detail on sample preparation and measurement techniques in the Raman analysis of ingle particles has been presented [36]. Various techniques have been applied in preparing mounting) specimens for analysis and generally those procedures followed in electron- and on-probe analysis are utilized. Samples for micro-Raman analysis do not, however, require oating by a conductive film (e.g., Al, C, Au) as is frequently required by the conventional ethods of microanalysis to dissipate specimen charging. Thin film overcoats, in fact, can le detrimental in the Raman microprobe measurement as these films (typically $\sim 20-40 \mathrm{~nm}$ hick) are sufficiently radiation-absorbing to induce sample heating, modification or even estruction.

(a) Substrate Selection. The selection of suitable substrate materials has been mportant for reasons alluded to earlier. To this point, the best choices have been highurity sapphire $\left(\alpha-\mathrm{Al}_{2} \mathrm{O}_{3}\right)$ and lithium fluoride (LiF) of single-crystal quality, commercially vailable in various forms (e.g., as optical windows) with a high-polish surface finish to ermit locating microscopic particles by light microscopy. They are used in the NBS work due to size constraints imposed by the collection system) in the shape of small rods (4 mm liameter, $6 \mathrm{~mm}$ long) held in an open, non-obstructing support collar. Sapphire is a weak laman scatterer and does not give rise to significant spectral interferences for particles arger than about 2-3 $\mu \mathrm{m}$. The material is well suited for the direct sampling (by impaction) if reactive aerosols (e.g., stack particulates from power plant emissions) because of its lurability and chemical inertness. Moreover, sapphire has high thermal conductivity and cherefore helps to minimize potentially destructive heating by serving as a heat sink to the rradiated particle. Lithium f7uoride is useful as a substrate material because it lacks "irst-order Raman activity and is obtained in high enough purity to be free from fluo'escence. In use, the surfaces of LiF substrates do, however, gradually deteriorate due to the attack by moisture in the ambient air, and the surfaces have been shown to react with iygroscopic particles, for example, causing chemical transformations to take place $[10,15]$.

Various other types of materials have been examined with respect to their utility as nicro-Raman substrates. Polished, highly-reflective metals (e.g., invar, stainless steels) can be used, although heating effects have been noted in some cases. Also, these are less desirable as a sampling surface for reactive aerosols.

Of some interest has been the evaluation of non-reactive polymeric membranes as supporting films for particle collections. Several types of plastic films are commonly ssed as filter media (e.g., Millipore, Nucleopore, Fluoropore, etc.) for the collection of ambient aerosol. These thin, microporous membrane filters (the nominal thickness of $0.8 \mu \mathrm{m}$ jore size Nucleopore filter is $\sim 10 \mu \mathrm{m}$ ) are not suitable as supporting films for particles to be analyzed in the Raman microprobe. For example, membrane filters of the polycarbonate and Teflon variety exhibit fairly complex Raman spectra with intense bands over the entire spectral range of interest. These serious interferences preclude the use of these membranes as substrates and require the removal of particle collections from such filters.

(b) Particle Mounting and Sampling Techniques. A number of techniques have come into use for the preparation of single particle mounts and the optimized sampling of suspended particles. These procedures have been described with particular reference to the characterization of particulates collected from the ambient air $[15,36]$ and from emissions by stationary sources [13]. Particle mounts on substrates are usually obtained from dispersions of bulk samples (e.g., filter-collected samples of airborne dusts). This involves transfer of a small, representative fraction of the bulk dust sample onto the substrate, with the aim to achieve a low density particle deposit devoid of extensive particle agglomeration. In 
some cases, individual particles of interest isolated from a bulk particulate sample are transferred to Raman substrates by the usual particle handling techniques used by particle analysts [37].

In the study of airborne particles and power plant stack particulates, we have attempted to optimize the collection of samples by in situ sampling of the particles suspended in the gas phase directly on Raman substrates. This second method of sample preparation involves the use of multi-stage cascade impactors $[24,38]$. For most size-classified samplings of aerosols we have used a five-stage impactor of the Battelle design modified to accept on each impaction stage the Raman substrate of standard design. By using this type of sampler and appropriate sampling times (to control the number density of particles collected, thus the mass loading) we have sought to achieve minimum quantity sampling in order to minimize all opportunities for changes to be induced (by particle-particle interactions) in the physical and chemical properties of the collected particles [39].

(c) Other Types of Microsamples. Frequently the sample under study is something other than a single microparticle or a collection of particles. Examples of the analysis of such specimens are drawn from NBS work in the investigation of fluid inclusions in minerals and the study of biological and pathological tissue sections.

Microscopic inclusions (solid, liquid, or gaseous phases) in geological samples can be examined in the microprobe in a straightforward way, although optimized sample preparation takes on special significance. Measurement limitations arise from constraints placed by the optical system on the location (in the mineral specimen prepared for analysis), geometry and dimensions of the inclusion. The inclusion must be observable without serious obstruction. A specimen prepared for normal microscopy (typically with a flat polished or natural face) is suitable for the microprobe measurement. Also, the host mineral should be reasonably transparent in the visible part of the spectrum (450 to $650 \mathrm{~nm}$ ), and the contents of the inclusion cannot be highly absorbing at the exciting laser wavelength. There are other intrinsic limitations (e.g., spectral) which will be indicated in the discussion of a specific example.

The examination of biological samples in the Raman microprobe presents another set of requirements on sample preparation. In the preparation of biological tissues for microanalysis, a major objective is that the in vivo distribution and concentration of elements and compounds in the different cellular and extracellular compartments is not changed. Important also is the correlation of composition with tissue morphology. Various techniques have come into routine use for the preparation of tissue sections (typically of thickness 2-10 $\mu \mathrm{m}$ ) suitable for elemental microanalysis by the conventional scanning electron microscope (SEM)/energy dispersive x-ray analysis (EDXA) approach. These methods - for soft tissue - usually consist of suitable procedures for the fixation, dehydration, embedding and sectioning of the tissue, and have been extensively described $[40,41]$. A number of the standard methods for the preparation of biological/pathological sections are fully compatible with the sample requirements for Raman microanalysis. Tissue sections prepared by cryosectioning techniques followed by air-drying, and standard sections embedded in paraffin have been satisfactory and have allowed non-destructive, in situ characterization of major molecular constituents. Measurement difficulties have been encountered in the investigation of tissue sections prepared by techniques involving exposure to some of the common fixatives, buffering agents and histological stains, as well as resulting from several of the common plastic embedding media. In general these tissue fixation and processing materials complicate the analysis either by causing absorption heating or by presenting serious spectral interferences (e.g., fluorescence).

\section{Measurement Procedures}

In the typical scheme of Raman microprobe analysis, the particle sample is first examined by 1 ight microscopy to single out and locate (with the aid of a thin aluminum film particle finder grid deposited on the substrate surface) individual particles of interest. The parameters observed include particle shape, size, color, transparency, surface roughness, and frequently also birefringence and extinction. Recognition of these properties can be of value in the subsequent Raman investigation. 
If a particle is colored, this may determine the choice of excitation frequency and e level of laser irradiance used. Examination in the optical microscope - supplemented optical micrographs taken before and after Raman analysis, to check on possible laser mage - is then followed by the microprobe measurement. The spectra presented in this per have been recorded by scanning the monochromator and plotting, on a strip chart corder, the analog signal proportional to the number of photon counts observed in a lected measurement time (i.e., the time constant). Typically, these spectra are obtained using irradiance levels ranging from several megawatts $/ \mathrm{cm}^{2}$ to values of a few kilowatts/ 2. (As a point of reference, the sun's irradiance - over the visible spectrum - amounts $\left.135 \mathrm{milliwatt} / \mathrm{cm}^{2}\right)$. The lowest practical power densities are often required for the obing of unknown, environmental particles or radiation-sensitive biological samples, for ample. Measurements generally require signal integration times from 1-4 seconds at scan tes of $50 \mathrm{~cm}^{-1} / \mathrm{min}$ to $10 \mathrm{~cm}^{-1} / \mathrm{min}$, respectively. A scan (with typical $3 \mathrm{~cm}^{-1}$ spectral solution) which covers the Raman spectral range from 0 to $3600 \mathrm{~cm}^{-1}$ (frequency shift, $\Delta v$ ) y therefore require from 1-6 hours of measurement time. The spectra of essentially lorless particles can be obtained at moderate to high power densities without deleterious fects on the sample. Such scans are recorded with integration times from 0.2 to 0.5 conds at scan rates of 200 to $100 \mathrm{~cm}^{-1} / \mathrm{min}$, respectively. Optimized instrument perfornce (achieved by careful optical alignment) furnishes spectra of high signal-to-noise tio with insignificant spectral interferences from either substrate (e.g., sapphire) issions or the elastic scattering component.

In many of our studies we find it useful to apply electron- and ion-probe analysis to termine elemental compositions as an aid to the interpretation of the Raman spectra. ray area scans which indicate the micrometer and submicrometer inhomogeneity of a particle ve been found to corroborate the results of micro-Raman measurements. Because of the gher elemental sensitivity (and other factors related to the fragmentation of compounds der ion beam bombardment) the interpretation of ion probe mass spectra and their correlaon with the Raman data is more complicated. The full integration of the micro-Raman chnique with the elemental microanalysis methods is actively pursued.

\section{Limitations of Raman Microprobe Analysis}

We will focus here on several aspects of the Raman microprobe measurement important to croanalysis. We will restrict this discussion to comments on (i) effective sampling lume and spatial resolution, (ii) laser-induced sample heating, (iii) sample fluorescence ad (iv) quantitation. With these comments we do not intend to convey a complete understandig of all the factors involved, but rather wish to identify those critical areas requiring arther elucidation if the prospects for effective analytical implementation of micro-Raman rectroscopy are to be enhanced.

\section{Effective Sampling Volume and Spatial Resolution}

An important experimental goal in microprobe analysis is to obtain measurements with as ecise and well known a spatial resolution as is possible. In Raman microprobe work, atial resolution may be defined by the intersection of the incident focused laser beam .e., the laser spot) and the field of view or the sampling volume of the collection tics. In the NBS microprobe, spatial resolution is determined by the particular configuraon of the focusing/light collection optics utilized in the fore-optical system (cf. gure 2).

The optical and geometrical factors that determine the effective sampling volume and ence the effective spatial (lateral and depth) resolution have been discussed for the case single microparticles supported by a substrate and for microscopic regions localized in below the surface of a heterogeneous bulk specimen $[9,23]$. Calculations show that for iscrete microscopic samples the diameter of the exit spatial filter closely determines the imensions of the collection volume. With a $140 \mu \mathrm{m}$ diameter exit spatial filter (i.e., size exit pinhole used for most measurements), the collection volume is ideally an ellipsoid ith axial dimensions of $8 \mu \mathrm{m}$ and $9 \mu \mathrm{m}$. Due to spherical aberrations and coma, the true epth (along the optic axis) of the collection volume is more like $12 \mu \mathrm{m}$ (in air). Thus, ie Raman signal originates from both the surface and some portion of the interior, or bulk,

the sample. For $\mathrm{F}$. ube volumes embedded in a host (e.g., the case of a microscopic fluid 
inciusion), the effective spatial resolution is less well defined owing to several optical effects and aberrations (distortions) of the sampling volume.

The degree of spatial filtering, determining the rejection of interfering scattering by the substrate, can be illustrated by the spectra shown in figure 4 . Two measurements were performed on a thorium oxide particle (a microsphere) of submicrometer size (diam. $0.8 \mu \mathrm{m}$ ), utilizing identical irradiance conditions (approx. $1 \mathrm{MW} / \mathrm{cm}^{2}$ ) but obtaining the signal without the exit pinhole in the second scan. The oxide displays a simple Raman spectrum [42] consisting of a single line (marked P) with shift $467 \mathrm{~cm}^{-1}$. The Raman bands characteristic of sapphire [43] are labeled S. The effect of limiting the collection volume of the ellipsoid by the size of the exit spatial filter is seen by comparing the signal-to-backgrounc ratios of the two spectra.

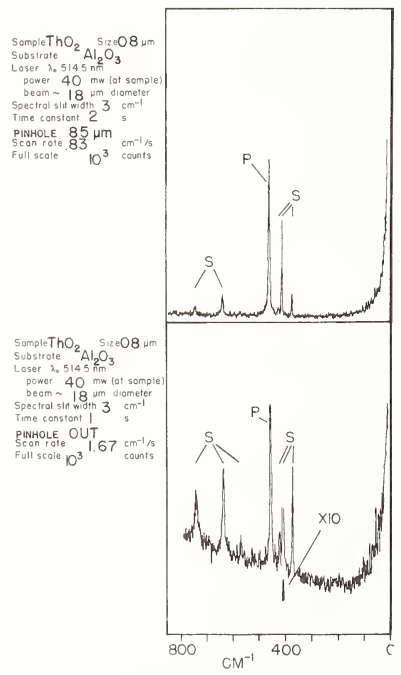

Figure 4 .

Raman spectra of a submicron thorium oxide particle showing the effect of spatial filtering on the spectral interference by the sapphire substrate. Bands marked F and $S$ derive from the particle and substrate, respectively. Upper spectrun obtained using $85 \mu \mathrm{m}$ diameter exit spatial filter, lower spectrum without the exit pinhole.

\section{B. Laser-Induced Sample Heating}

Strong absorption of the focused laser radiation by colored samples gives rise to samplt heating and may lead to their thermal decomposition. Measurements must therefore be madk under irradiance conditions commensurate with preserving the integrity of the sample. The microprobe systems in use have been designed so that the signal-to-background ratio is essentially independent of irradiance level. This means that the power density on the sample can be reduced, but this requires that measurement times are increased.

The case of sample heating for radiation absorbing spherical microparticles has beet considered $[9,23,44]$. From model calculations, estimates have been derived for the transient and steady state temperature reached by optically absorbing spherical particles immersed ir a host (e.g., air or $\mathrm{Al}_{2} \mathrm{O}_{3}$ ). Results show that heating effects become less severe as the particle size decreases (which is also born out by experiment) and that a sapphire host, for 
ample, is three orders of magnitude more efficient as a heat sink than air. The calculaons indicate also that destructive heating of microparticles can be attributed to highly calized absorption and large temperature gradients in the particle. These effects can be ought on by light-absorbing surface layers or microscopic inclusions. In these cases, e heat-sinking properties of the substrate material are likely to be ineffective.

Laser-induced sample heating usually leads to noticeable spectral changes. Bands tend shift to lower wavenumbers and broaden and sample changes, such as phase transitions or mpositional modifications, can occur. Not much is known about real energy density threshds for significant absorption-induced perturbations of microsamples. Complete sample eakdown (e.g., melting, vaporization) by simple absorption heating sets an upper limit on e allowable intensity of the focused beam.

The spectra of figure 5 were obtained in two successive measurements under conditions low and high irradiance, employing $514.5 \mathrm{~nm}$ excitation. The sample is a $\sim 3 \mu \mathrm{m}$ particle lated from a bulk sample of airborne dust. The unknown particle is identified as sodium trate $\left(\mathrm{NaNO}_{3}\right)$ with reference to published Raman data for this salt [45]. The upper ectrum was obtained first at an irradiance of $5.7 \mathrm{~kW} / \mathrm{cm}^{2}$; the lower spectrum was then tained at $0.32 \mathrm{MW} / \mathrm{cm}^{2}$. The most pronounced difference in the two spectra consists of the o broad bands in the $1200-1700 \mathrm{~cm}^{-1}$ region and the appearance of a much higher luminescence vel in the high irradiance spectrum. Other differences are noted in both the intensity $d$ in the spectral definition of the bands, with frequency shifts $98,185,724,1068$, and $85 \mathrm{~cm}^{-1}$, attributed to sodium nitrate. The two prominent, broad bands in the lower ectrum, centered at 1350 and $1600 \mathrm{~cm}^{-1}$, are associated with the presence of a form of lymeric (also called graphitic or elemental) carbon not detected in the low irradiance ectrum. A repeat scan (not shown) of the particle under the same low irradiance condions showed the same spectral features as the high irradiance scan. In these spectra we e the result of the absorption-induced conversion of an environmental carbonaceous material minor constituent of the particle, likely surface-adsorbed) to a form of polymeric carbon th graphitic structure $[14,46]$.

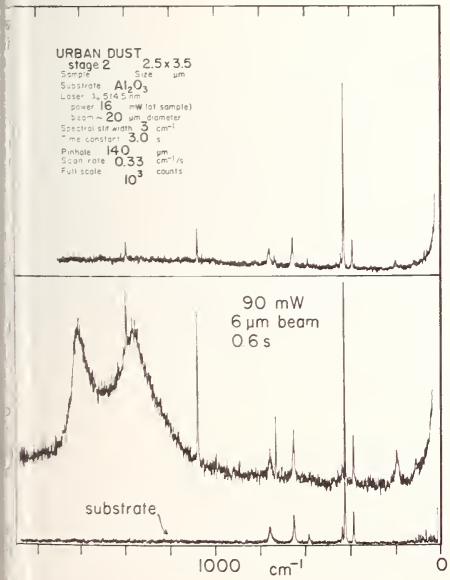

Figure 5 .

Effect of laser irradiance (watts $/ \mathrm{cm}^{2}$ ) on the spectrum of a microparticle of sodium nitrate $\left(\mathrm{NaNO}_{3}\right)$ in urban air particulate dust. Power density at sample: top, 5.7 $\mathrm{kW} / \mathrm{cm}^{2}$; bottom, $0.32 \mathrm{MW} / \mathrm{cm}^{2}$. Shown also is the (interfering) spectrum of the sapphire substrate. 
The carbon bands, so frequently detected in the spectra of pollution particles, have beer of special interest to us from two points of view. First, there is the question of the source and nature of this carbon material in the environment and, second, the possibility of the conversion of any associated organic (i.e., hydrocarbon) material to a form of polycrystalline graphite as a result of the high-irradiance microprobe measurement. These questions were examined following numerous experimental observations of the appearance of these bands in the spectra, their sensitivity to laser irradiation, as evidenced by readily perceptible changes in the shape and the relative intensities of the two bands.

In a series of modeling experiments described elsewhere [14], laboratory-prepared particle samples of soluble anhydrite $\left(\mathrm{CaSO}_{4}\right.$, known to undergo a phase transition at $\sim 300$ ${ }^{\circ} \mathrm{C}$, converting to the insoluble anhydrite form) were coated with thin layers of various forms of carbon (e.g., vapor-deposited vitreous carbon) or carbonaceous organic material (e.g., petroleum hydrocarbons) to relate the spectral profile of the carbon bands to both the nature of the surface film and the irradiance conditions of the measurement. Figure 6 shows spectra of $\mathrm{CaSO}_{4}$ microparticles coated, in one case (spectrum B) with a (liquid) film of $\mathrm{n}$-hexadecane. The two spectra were obtained under identical irradiance conditions. The spectrum of the particle coated with the hydrocarbon liquid exhibits the familiar spectral features assigned to polymeric elemental carbon and indicates that in this measurement the organic film has been converted to a form of graphitic carbon. In other Raman work on carbonaceous particles sampled from polluted air, this form of carbon has been identified with soot [47]. Thus, the two distinct bands have developed as a consequence of the decomposition of the hydrocarbon with the formation of a carbon residue of microcrystalline structure [46]. Continued exposure of this sample to laser irradiation brought on a partial transformation from the soluble to the insoluble form of anhydrite. This conversion is thought to have occurred by the heating of the particle due to the optically absorbing carbon film that had formed.

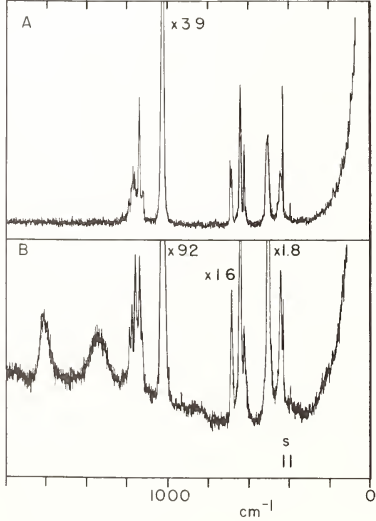

Figure 6.

Raman microprobe spectra of particles of soluble anhydrite $\left(\mathrm{CaSO}_{4}\right)$ with and without a coating of $n$-hexadecane. A. Non-coated particle. Measurement parameters: particle size, $6 \times 16 \mu \mathrm{m}$; substrate, $\alpha-\mathrm{Al}_{2} \mathrm{O}_{3}$; laser, $\lambda_{0}=514.5 \mathrm{~nm}$; power, $90 \mathrm{~mW}$ (at sample); beam spot, $\sim 6 \mu \mathrm{m}$ diameter; spectral slit width, $3 \mathrm{~cm}^{-1}$; time constant, $0.5 \mathrm{sec}$; scan rate, $100 \mathrm{~cm}^{-1} / \mathrm{min}$; intensity, 1000 counts full scale. B. Particle coated with hydrocarbon liquid; size, $8 \times 12 \mu \mathrm{m}$. Measurement conditions as for $A$, except: time constant, $1.0 \mathrm{sec}$; scan rate, $50 \mathrm{~cm}^{-1} / \mathrm{min}$. 
Fluorescence assumes major importance in the selection of excitation wavelength. occurs when one or more constituents of the observed system (e.g., a single particle or biological tissue section) absorb part of the incident light and then emit some of this sorbed energy. The fluorescence emission can be many orders of magnitude stronger than man scattering from the sample. Thus, fluorescence from sample impurities or contaminants n provide severe background interference to microprobe measurements. In the analysis of vironmental particulates, fluorescence can be attributed to absorption by organic combuson residues (present as contaminating films) or other types of adsorbed materials. In ganic and biological samples, this fluorescence can be very strong.

The fluorescence in these measurements manifests itself as a broad continuum in the man spectrum of many samples. With microsamples, there is not much that one can do to duce the fluorescence background to manageable levels, such as by sample "clean-up" as in cro-Raman work. Sometimes, however, the fluorescence background decays with time to the int at which a suitable signal-to-noise level is reached. In rare cases can one subtract e fluorescence background to yield a reliable Raman spectrum.

In recent work we have more systematically examined the problem of sample fluoresnce. As part of a broader study, the spectra of microsamples of a series of polynuclear omatic hydrocarbons (PAHs) were obtained. The object was to evaluate the analytical tential of micro-Raman spectroscopy in the trace characterization of solid organic llutants [48]. The PAHs were measured as single microparticles, generally of size 2-10 , and include representative members of the 3-ring (e.g., phenanthrene) and 4-ring (e.g., rysene, pyrene, fluoranthene) systems. The fluorescence levels were examined in the ectra excited at three laser wavelengths - $514.5 \mathrm{~nm}$ (green), $568.2 \mathrm{~nm}$ (yellow), and $7.1 \mathrm{~nm}$ (red). In general, excitation with $514.5 \mathrm{~nm}$ radiation under the lowest practical radiance conditions (typically $2 \mathrm{~mW}$ at sample in a $20 \mu \mathrm{m}$ beam spot) furnishes spectra aracterized by high, broad-band fluorescence levels. In these situations, a useful ectrum cannot be obtained. Excitation at $568.2 \mathrm{~nm}$ results in spectra that have apprecily lower background levels, indicating much diminished fluorescence emission at this velength. With $647.1 \mathrm{~nm}$ excitation, fluorescence from these compounds is virtually n-existent.

A result from these investigations is the spectrum shown in figure 7 for a subnanogram crocrystal of fluoranthene excited with the yellow laser line. The spectrum has excellent gnal-to-background due to the absence of fluorescence. This inconsequential background is en further diminished in the red. With green line excitation, overwhelming fluorescence ission was encountered from these particles upon which the strongest bands in the spectrum iuld barely be detected. The sharp doublet at the high frequency end (i.e., at higher ivenumber shifts) is not attributed to the spectrum of fluoranthene but arises from the ruby $\mathrm{r}_{2} \mathrm{O}_{3}$, present as a trace impurity) fluorescence in sapphire. This fluorescence doublet : lines of ruby) appears at wavenumber shifts $3167 \mathrm{~cm}^{-1}$ (R2 1 ine, $692.9 \mathrm{~nm}$ ) and $3196 \mathrm{~cm}^{-1}$ ?] line, $694.3 \mathrm{~nm}$ ) with $568.2 \mathrm{~nm}$ spectral excitation. The ruby doublet from sapphire ubstrates represents a more serious interference in spectra excited with the red line, for ien the two intense bands appear in a highly diagnostic region (e.g., for sulfates, carbonces) of the spectrum, at Raman shifts $1021 \mathrm{~cm}^{-1}$ and $1050 \mathrm{~cm}^{-1}$. This makes necessary the e of lithium fluoride substrates for which this spectral interference does not arise. 


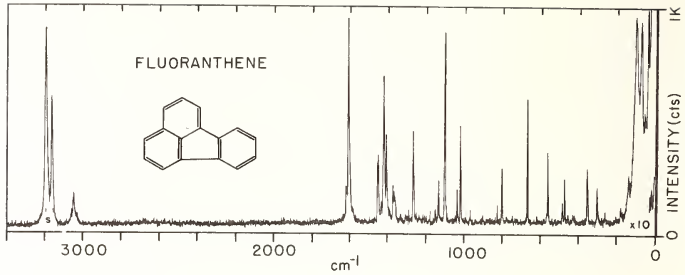

Figure 7. The spectra of fluorescence sensitized polynuclear aromatic hydrocarbons (PAHs). Microprobe spectrum of fluoranthene $\left(\mathrm{C}_{16} \mathrm{H}_{10}\right)$. Measurement parameters: particle size, $7 \times 9 \mu \mathrm{m}$; substrate, $\alpha-\mathrm{Al}_{2} \mathrm{O}_{3}$; laser, $\lambda_{0}=568.2 \mathrm{~nm}$; power, $5 \mathrm{~mW}$ (at sample); beam spot, $\sim 18 \mu \mathrm{m}$ diameter; spectral slit width, $3 \mathrm{~cm}^{-1}$; time constant, $0.2 \mathrm{sec}$; scan rate, $200 \mathrm{~cm}^{-1} / \mathrm{min}$; intensity, 1000 counts full scale. Bands marked $S$ arise from the fluorescence of the substrate.

The spectra of these structurally closely related PAHs (e.g., the compounds in the 4-ring system) are sufficiently different to allow unequivocal identification of these molecular solids. 0ther studies on selected isomeric compounds of the 5-ring system (e.g., benzo[a]pyrene, perylene) indicate a similarly high specificity of the Raman spectrum. A detection limit (with the Raman microprobe in its present state of development) of 10-100 pg is indicated for many of these environmentally significant hydrocarbons.

\section{Quantitation}

The relationships for quantitative analysis in conventional macro-Raman spectroscopy are based on the observation that the Raman scattering intensity is proportional to the concentration of the species which generates the line in the spectrum. The band intensities which are meaningful for quantitation of Raman data are the relative integrated intensities. Raman signal intensities from different solid samples cannot ordinarly be directly compared, because the scattering phenomena are complicated and instrumental factors play a decisive role. Despite these complications, in much of contemporary Raman work (e.g., solution- and gas-phase analysis), reliable quantitative results can be obtained by reference to internal or external calibration standards [3]. This approach compensates for differences in matrix effects (e.g., bulk optical properties), sample geometry (relative to excitation/collection configuration) and instrumental factors.

When the conventional considerations basic to quantitation in macro-Raman spectroscopy are extended to the Raman scattering of light by microscopic samples, formidable complications arise. The fundamental difficulties result from the lack of an adequately developec theory of inelastic (Raman and fluorescence) scattering from microparticles [19]. The detailed effects that need to be considered in any model for inelastic scattering are complicated, and their elucidation is the subject of ongoing work by several investigators $[19,49-52]$. Any approach to the quantitative estimation of species concentrations in microparticles must take these detailed effects into account.

The theoretical foundations for inelastic scattering by microscopic particles will not be reviewed here. However, some comments on this topic are appropriate in outlining the relationship to quantitation in Raman microprobe analysis. The theories show that the morphological (e.g., size and shape) and optical properties (e.g., refractive index) of the particle profoundly influence the Raman scattering. Adding further to these effects on the signal are factors related to the distribution of active molecules within the particle. These properties strongly influence the local electric field which excites molecular 
ansitions and the angular distribution, polarization, and intensity of the emitted adiation. From an experimental point of view, particle orientation effects, angle of oservation and polarization phenomena must be considered in conjunction with the optical ssign of the instrument and the instrument function itself. Thus, one can anticipate a mplicated relation between Raman intensity and component concentration. In the attempts ade to quantify several of these effects from exact Mie (elastic) and Raman (inelastic) sattering theory, they have been considered to arise from the influence of the particle sundary both upon the internal or local electric field at the incident frequency and upon ve emissions at the shifted frequency rather than from any alterations of the molecular ansitions [50].

The theoretical treatments which have been advanced all consider the interaction of the cident radiation (or electric) field with spherical particles [49-52]. This has led to alculations for Raman scattering by molecules embedded in isotropic, dielectric spheres and cludes the case of light scattering by structured (layered) spheres. These theories ttempt to estimate the properties of the electric fields within the particle (i.e., the ielectric response of the material) and their effects on the properties of the inelastically zattered field. It has been proposed that the electric fields within the particle are ighly structured and vary in polarization and strength with particle size, shape, and efractive index. The treatments have further recognized that, since inelastic scattering $y$ molecules embedded in microparticles is stimulated by the local internal field and since e local dissipation of heat for particles also arises from interaction with this field, 3ne two processes of optical absorption and scattering are related [50-52]. Thus, the Eattering dependence upon particle properties is qualitatively similar to the dependence $f$ the absorption upon these properties.

In calculations of the efficiency for optical absorption by microscopic particles, the heoretical prediction of resonances in the efficiency factors for absorption has led to he formulation of a corresponding fine structure in the Raman scattering from microarticles, described in terms of internal field resonance effects that parallel those known rom elastic light scattering (or Debye-Mie scattering) theory [51-53]. Since these esonances in the internal fields depend on the frequency of the exciting radiation, diferent regions of the inelastic scattering spectrun can have quite different intensities. urthermore, the calculations predict that these peaks in both the elastic and inelastic cattering are a sensitivite function of the optical absorption and the exact size of the article. An accurate prediction of these resonance effects on the Raman spectrum of icroparticles that do not fit the model of a perfect sphere is exceedingly difficult, and las not been made.

A preliminary experimental investigation (514.5 $\mathrm{nm}$ excitation) of the intensity of the lastic and inelastic ( $467 \mathrm{~cm}^{-1}$ band, cf. fig. 4) scattering from microspheres of thorium xide, ranging in size (diameter) from 1 to $10 \mu \mathrm{m}$, has been performed with the Raman microrobe [9]. Results of these measurements are shown in figure 8 . Plotted are scattered ntensity (Mie and Raman) versus particle diameter. The theoretical curve predicted from xact Mie scattering theory (with intensities integrated over a scattering angle from $64-120^{\circ}$ ) is shown in the upper graph with the experimental points from a large number of bservations closely falling about this solid curve. The experimental points are in quite ood agreement with the prediction of the Mie theory, showing a $r^{2}$ dependence (where $r$ is he particle radius) of the scattered intensity on particle size. The fine structure ssociated with the Mie scattering curve results from the resonances. With respect to the aman data, in a first approximation one would expect the inelastic scattering intensities o be proportional to the number of scattering molecules contained in the particle, or imply proportional to the volume of the particle. This expected behaviour is indicated $y$ the solid line, drawn to show this $r^{3}$ dependence. The experimental Raman intensities verall show this trend with particle volume. However, there are deviations from this imple relationship which are suggestive of the resonance structure predicted by current heory, and similar to that noted for the Mie intensity curve [49-53]. Experimental work s continuing in this area to firmly establish the existence of these resonances in the aman scattering intensity both for narrow intervals of the size parameter, or for a fixed article size, over a narrow excitation wavelength interval. 


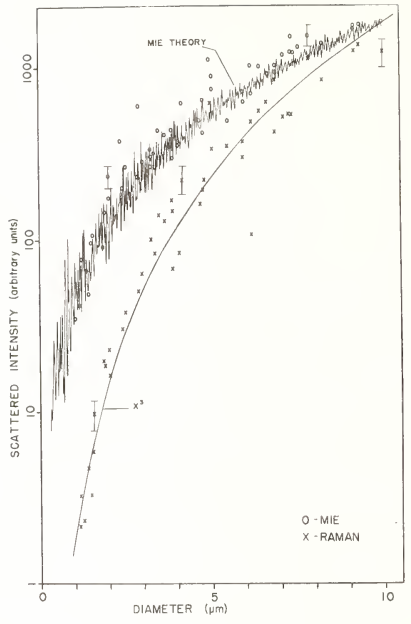

Figure 8.

Mie (elastic) and Raman (inelastic) scattered intensities as a function of particle size for thorium oxide $\left(\mathrm{ThO}_{2}\right)$ microspheres. Raman intensities measured for the band with shift $467 \mathrm{~cm}^{-1}$ (cf. fig. 4). Solid curves calculated from first principles; points are experimental.

Applications of Raman Microprobe Spectroscopy

\section{A. Areas of Investigation}

We will present here selected examples of investigations conducted with the NBS microprobe in several areas of microanalysis. Research along similar lines has been performed with the MOLE and literature citations to this work have been given $[7,11,12]$. Earlier in the discussion of this paper, reference was made to the general problem areas that have been investigated by micro-Raman techniques.

The NBS micro-Raman work has focused on a diversity of problems related to environmental particulate pollution, with emphasis on the spectroscopic characterization of airborne particles and atmospheric aerosols [13-15,36]. Interest has centered on the identification of the principal sulfur- and nitrogen-bearing species, such as sulfate $\left(\mathrm{SO}_{4}{ }^{2-}\right)$, nitrate $\left(\mathrm{NO}_{3}{ }^{-}\right)$and ammonium $\left(\mathrm{NH}_{4}{ }^{+}\right)$in the coarse $(>2 \mu \mathrm{m})$ size fraction of the particle collections. Samples (of mass in the subnanogram range) composed of submicrometer-size aerosol collected from the South Pole atmosphere have been analyzed to correlate results with elemental data obtained by x-ray microanalysis [15]. Microparticulate emissions from power plants, i.e., fly ash and stack aerosol, have been studied to discern the molecular identities of those species whose major elements were determined by $x$-ray microanalysis $[13,54]$. Carbonaceous matter was found to be associated with many of the environmental particles analyzed, and a major fraction of this material could be attributed to a form of elemental carbon with structures similar to polycrystalline graphite. These studies of environmental particles have furnished new insights on particle transformations, sampling effects on the chemistry of inicroparticles, and a better assessment of the effects of the microprobe measurement on the sample and the spectroscopic information that can be obtained. In the area of micromineralogy, non-destructive in situ analysis of individual phases in fluid inclusions in geological samples have furnished new results of interest $[9,55]$. Individual microscopic 
rystals and fibers of several of the common asbestos minerals (i.e., sheet and chain ilicates) have been characterized by microprobe measurements $[16,54]$. The aim was to lucidate any spectral differences that might be ascribed to the distinct fibrous and nonibrous morphologies of these minerals. In feasibility studies, micro-Raman spectroscopy las been evaluated as a sensitive and specific detection method for organic fractions eparated by liquid chromatography [48]. In this area of trace organic analysis, the dentification of structurally similar polynuclear aromatic hydrocarbons (PAHs), many of which are potent carcinogens, is of importance to pollution studies. The possibilities for ibrational analysis at the microscopic level in biology and medicine are very exciting. 'reliminary investigations in this area have resulted in demonstrating nondestructive snalysis of thin sections of soft and hard biological tissues $[17,18]$. Here we will briefly rention the unique information obtained in the study of bone and tooth mineralization 17]. A second example is provided by microprobe measurements on standard sections of ntact pathological tissue leading to the identification of organic, microparticulate :oreign-body inclusions [18].

The examples we have chosen to discuss from the above areas of application are intended co provide a perspective of the practical utility of the technique and the prospects for Further analytical implementation in the future.

\section{Study of Airborne Particles}

The analysis of airborne particles is important in many fields of human endeavor, including concerns related to pollution monitoring, global climate modification, health hazard evaluation, and contamination control. In our studies on particles collected from che ambient air, we demonstrated detection and identification of a broad range of substances present in the atmospheric environment. The principal inorganic species for which spectroicopic characterization in microparticles is sought include the common minerals (e.g., silicates), oxides, carbonates, sulfates, sulfites, nitrates, phosphates, and ammonium salts. Since a number of these species also exist as water-soluble compounds (e.g., $\left.\left(\mathrm{NH}_{4}\right)_{2} \mathrm{SO}_{4}, \mathrm{~Pb}\left(\mathrm{NO}_{3}\right)_{2}\right)$ they may also occur in the dissolved state of liquid microparticles, and, in fact, are commonly encountered either in solution or extensively hydrated, particularly when such hygroscopic species are associated with the fine $(<2 \mu \mathrm{m})$ size fraction of atmospheric aerosol (e.g., acid aerosol). In the analysis of liquid microparticles, the nicroprobe spectra can furnish unique information (not obtained by conventional microprobes which require the sample to be placed in a vacuum) on the specific chemical states of dissolved species, their extent of dissociation, and in addition can provide some measure of relative concentrations. In measurements on microdroplets of laboratory-generated sulfuric acid aerosol, we have shown capability for the detection of dissolved molecular ions [36] and can illustrate these results with the spectra shown in figure 9 . In these experiments we have examined the transformation of individual (liquid) $\mathrm{H}_{2} \mathrm{SO}_{4}$ microparticles to solid, nicrocrystalline $\left(\mathrm{NH}_{4}\right)_{2} \mathrm{SO}_{4}$ by reaction with ammonia vapor. This conversion is one of considerable interest to the study (e.g., mechanisms, kinetics) of atmospheric reactions involving the neutralization of ambient acid aerosol. 


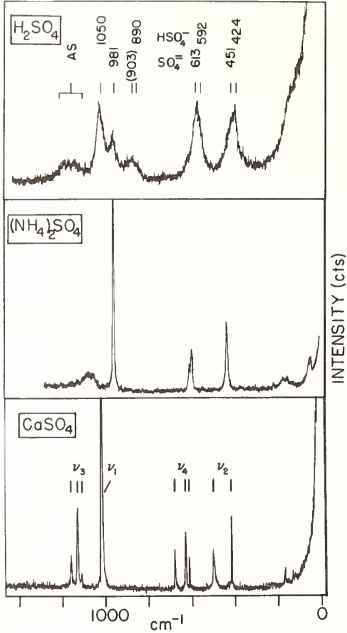

Figure 9.

Raman microprobe spectra of sulfate species in liquid and solid microparticles. (i) Spectrum of a microdroplet $(\sim 30 \mu \mathrm{m}$ diameter) of sulfuric acid. Indicated are the characteristic Raman shifts $\left(\mathrm{cm}^{-1}\right)$ of $\mathrm{HSO}_{4}{ }^{-}$and $\mathrm{SO}_{4}{ }^{2-}$ ions in the aqueous solution phase. (ii) Spectrum of a $\sim 8 \mu \mathrm{m}$ particle of crystalline ammonium sulfate. (iii) Spectrum of a $\sim 5 \mu \mathrm{m}$ particle of anhydrite. The $\nu_{i}$ designations indicate the frequencies of the fundamental internal vibrational modes of the sulfate ion in $\mathrm{CaSO}_{4}$.

The upper spectrum of figure 9 demonstrates the detection and characterization in the liquid phase of the various sulfate species in a single microdroplet (size, $\sim 30 \mu \mathrm{m}$ diameter) of sulfuric acid. In concentrated $\mathrm{H}_{2} \mathrm{SO}_{4}$, all three species - undissociated $\mathrm{H}_{2} \mathrm{SO}_{4}, \mathrm{HSO}_{4}^{-}$, and $\mathrm{SO}_{4}{ }^{2-}$ - are detectable. The Raman shifts characteristic of the $\mathrm{HSO}_{4}^{-}$and $\mathrm{SO}_{4}{ }^{2-}$ ion in solution are indicated. The weak band at $903 \mathrm{~cm}^{-1}$ is assigned to the undissociated $\mathrm{H}_{2} \mathrm{SO}_{4}$ molecule [56]. The middle spectrum was obtained from a microcrystal ( $8 \mu \mathrm{m}$ in size) of ammonium sulfate, formed by "gas phase titration" whereby a microdroplet of $\mathrm{H}_{2} \mathrm{SO}_{4}$ was exposed to trace concentrations ( $\sim 50 \mathrm{ppm})$ of ammonia $\left(\mathrm{NH}_{3}\right)$ [36]. All of the predicted Raman modes are present [57]. The predominant band is due to the symmetric sulfate stretch with characteristic Raman shift $976 \mathrm{~cm}^{-1}$. The other bands appear at the expected frequencies (cf. Table 1). The lower spectrum was obtained in other studies; it is a reference spectrum of the mineral anhydrite obtained from a $5 \mu \mathrm{m}$ particle. For this sulfate, the symmetric stretch $\left(v_{1}\right)$ is found at $1018 \mathrm{~cm}^{-1}$. The experimentally observed frequencies of the other fundamental internal vibrational modes (designated $v_{3}, v_{4}$, and $\nu_{2}$ ), which are those predicted from theory, are indicated (cf. Table 1) [58]. From spectroscopic studies - such as these described above - of known materials and compounds, reference spectra are obtained to aid the interpretation of unfamiliar spectra. The spectra of microsamples of known identity and composition also serve to establish limits of detection for classes or specific types of compounds of interest. 
Table 1. Raman shifts of sulfates in the solid phase.

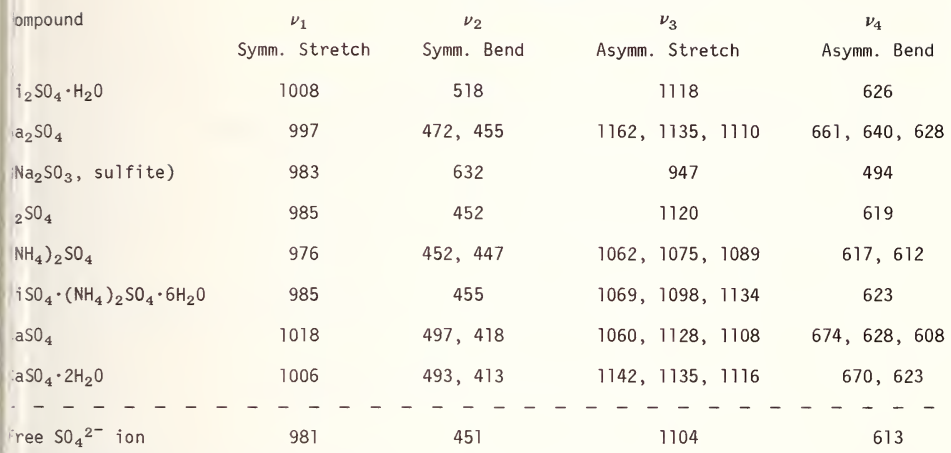

$\mathrm{SO}_{4}{ }^{2-}$ Frequency Shift, $\mathrm{cm}^{-1}$

ompound

$\mathrm{i}_{2} \mathrm{SO}_{4} \cdot \mathrm{H}_{2} \mathrm{O}$

451

1104

613

The Raman shifts observed from microparticles of several common crystalline sulfates ire given in Table 1 [59] and are representative of the internal vibrational modes ( $S-0$ itretching or bending) of the $\mathrm{SO}_{4}{ }^{2-}$ ion of tetrahedral symmetry. The frequencies of external or lattice modes characteristic of the crystal lattice are not given. Sulfates and iulfites ( $\mathrm{cf}$. $\mathrm{Na}_{2} \mathrm{SO}_{4}$ vs. $\mathrm{Na}_{2} \mathrm{SO}_{3}$ ) can readily be distinguished, as the sulfite ion has jyramidal symmetry. Similarly, a comparison of the frequency shifts for the sulfate ion in inhydrite $\left(\mathrm{CaSO}_{4}\right)$ and in gypsum $\left(\mathrm{CaSO}_{4} \cdot 2 \mathrm{H}_{2} \mathrm{O}\right)$ allows distinct hydration states to be assigned to these solids $[58,60]$. The Raman shifts given for the free $\mathrm{SO}_{4}{ }^{2-}$ ion correspond :o the frequencies of the fundamental modes of the ion in solution (cf. figure 9) [59].

For the analysis of airborne particles we have used samples collected by impactors as vell as filter samples obtained in the use of conventional high-volume air sampling equipnent. In this work, described in detail elsewhere, we have reported critical observations regarding the sampling process [36]. Evidence was presented for the chemical transformation If particles in cases where bulk sample collections were made over extended periods of sampling. Specifically, particle reactions could be inferred that resulted from exposure of the filter-collected sample to ambient atmospheric acid aerosol. These observations indicated the importance of unambiguous sampling and suggested a useful application of the Raman microprobe to the study of the sampling process itself. Spectroscopic evidence for this kind of an effect is shown in figure 10. The particle analyzed was isolated from a sulk sample of St. Louis urban dust which had been collected on a glass fiber filter employing a high-volume air sampler. Qualitative comparison of the spectrum of the particle with reference spectra $[58,61]$ identifies the unknown to principally consist of calcite $\left(\mathrm{CaCO}_{3}\right)$ as the major component and insoluble anhydrite ( $\mathrm{CaSO}_{4}$, cf. fig. 9) as a minor component. Al1 five Raman active modes of $\mathrm{CaCO}_{3}\left(1432,1088,714,283\right.$, and $156 \mathrm{~cm}^{-1}$ ) are present [61]. The $1432 \mathrm{~cm}^{-1}$ band is almost completely obscured by the broad spectral feature in the 1200 to $1700 \mathrm{~cm}^{-1}$ region. This feature entails two band maxima at $\sim 1350$ and $\sim 1600 \mathrm{~cm}^{-1}$, assigned to the carbon bands characteristic of polycrystalline graphite. They arise from the presence of (or contamination by) a form of carbon similar to soot. Bands similar to these have been observed in the spectra of many different types of environmental particles (cf. figure 5). In some of the spectra obtained from airborne dust particles, these were the only discernible features. The presence of $\mathrm{CaSO}_{4}$ as a minor constituent is indicated by the band at $1018 \mathrm{~cm}^{-1}$ (cf. Table 1). This feature is the most prominent one in the spectrum of $\mathrm{CaSO}_{4}$, and on the basis of its intensity and the relatively high spectral background we do not expect to be able to discern the other characteristic bands. We have interpreted the presence of $\mathrm{CaSO}_{4}$ to indicate the partial transformation of $\mathrm{CaCO}_{3}$ by acid aerosol [36]. 


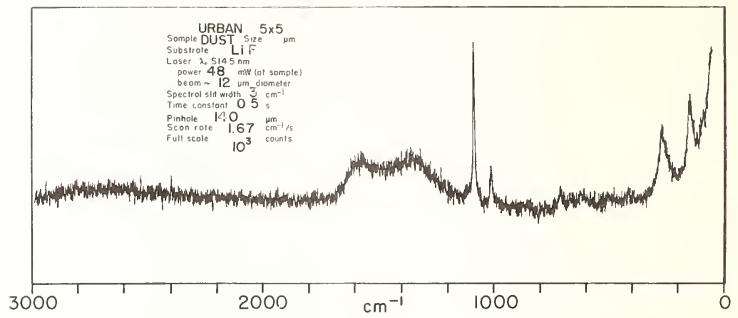

Figure 10. Raman spectrum of a particle of calcite $\left(\mathrm{CaCO}_{3}\right)$ in urban air particulate dust. Measurement conditions are indicated.

\section{Microparticulate Organics}

Airborne particles can also be of organic origin and composition (e.g., pesticide dusts) or they may consist of an inorganic core (e.g., oxides produced at high temperatures, -fly ash) with a surface layer of an organic material formed by the condensation of organics in the vapor phase (e.g., polycyclic hydrocarbons from the combustion of fossil fuels). A large fraction of the organic material in ambient air is found with atmospheric particles in the respirable size $(\widetilde{<} 10 \mu \mathrm{m})$ range [62]. Many of these compounds are suspected carcinogens. Raman microprobe techniques are eminently suited for the characterization of microparticulate organics and polymers, or hydrocarbon films $[18,36,48]$. Organic compounds give informative, diagnostically useful Raman spectra [63].

Many different types of organic compounds have been examined in the Raman microprobe [8]. These studies have established practical limits of detection for various classes of organics and have provided some insight into potential measurement difficulties as well as problems in the interpretation of the spectra - due to their complexities - that one can expect to encounter with organic molecules. Two examples from these measurements are shown in figures 11 and 12, for microparticles of laboratory-grade materials. Certain organochlorine pesticides have been recognized as persistent environmental chemicals and are known to be transported in air. They are colorless, crystalline solids, and are therefore not expected to heat or modify in the microprobe measurement, nor would these compounds be expected to give rise to fluorescence interferences. The chlorocarbon insecticides kepone and mirex, for example, are closely related compounds and have many similar properties [13]. Their spectra (fig. 11) permit these two pesticides to be readily distinguished. This shows that even though closely related molecular solids have similar spectra, spectral regions can usually be found which allow positive discrimination between the various possibilities. 


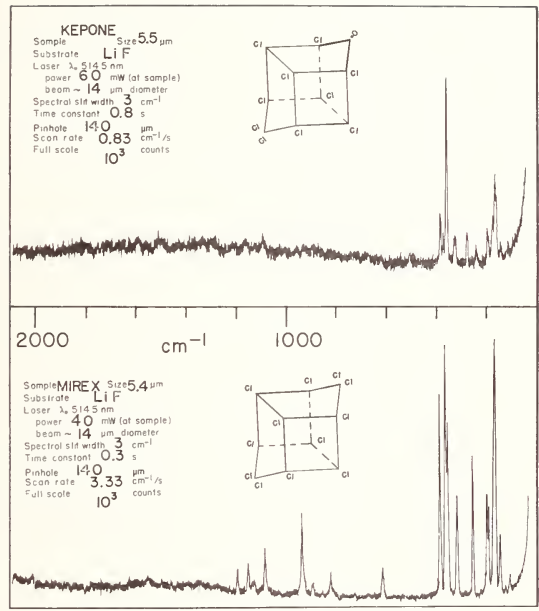

Figure 11. Reference spectra of microparticles of the chlorocarbon insecticides kepone and mirex. 


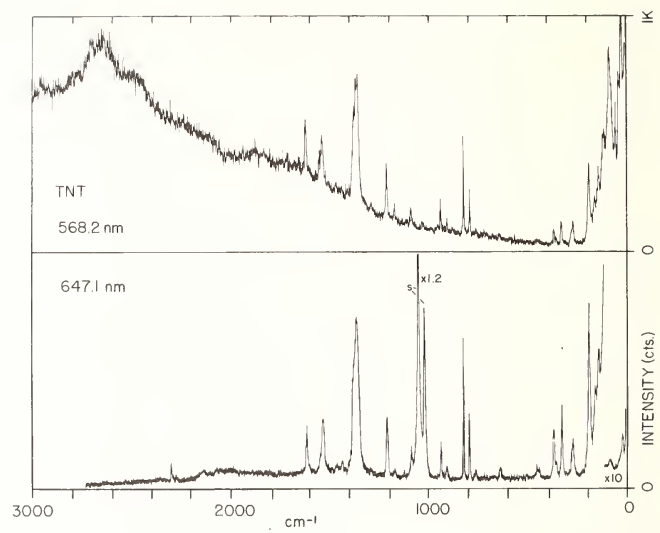

Figure 12. Spectra of microcrystals of trinitrotoluene (TNT) excited at two laser wavelengths. Measurement parameters: Top - particle size, $20 \times 60 \mu \mathrm{m}$; power, $8 \mathrm{~mW}$ (at sample); beam spot, $\sim 16 \mu \mathrm{m}$ diameter; time constant, $2.0 \mathrm{sec}$; scan rate, 50 $\mathrm{cm}^{-1} / \mathrm{min}$. Bottom - particle size, $15 \times 25 \mu \mathrm{m}$; power, $30 \mathrm{~mW}$ (at sample); beam spot, $\sim 12 \mu \mathrm{m}$ diameter; time constant, $2.0 \mathrm{sec}$; scan rate, $50 \mathrm{~cm}^{-1} / \mathrm{min}$. For both spectra - spectral slit width, $3 \mathrm{~cm}^{-1}$; pinhole, $140 \mu \mathrm{m}$; intensity, 1000 counts full scale. Particles supported by a sapphire substrate. Bands marked $S$ in lower spectrum are due to substrate fluorescence.

Spectral changes induced by laser irradiance are frequently observed in the analysis of organic microparticles. However, these changes cannot always be fully explained as resulting from thermally-induced modifications of the sample, but may result from photodecomposition (e.g., photooxidation, photolysis), as many organic molecules are unstable to high-intensity visible laser radiation. The spectra of figure 12, of two microcrystals of trinitrotoluene (TNT explosive, straw-yellow colored) excited with the yellow and red line of the laser, illustrate this kind of observation. The bands in the TNT spectrum arise from vibrations of the pheny $\left(\mathrm{C}_{6}\right)$ ring, $\mathrm{C}-\mathrm{H}$ and $\mathrm{C}-\mathrm{N}$ bonds, and vibrations of the $\mathrm{NO}_{2}$ groups [63]. No spectra could be obtained with green line $(514.5 \mathrm{~nm})$ excitation owing to instantaneous sample decomposition (melting point $82{ }^{\circ} \mathrm{C}$ ). The top spectrum - obtained under lowirradiance conditions - shows a slowly progressing sample modification, evidenced by the changing or developing background during the course of the measurement. This luminescent background cannot be attributed to thermal radiation (incandescence), for the microcrystal is still at a low (nonincandescent) temperature well below its melting point. From this observation we cannot exclude - in this and other such examples - the possibility of the optically-induced formation of a fluorescing species. Since the substrate material is sapphire, we note in the bottom spectrum the fluorescence interference (cf. fig. 7) from trace amounts of chromium $\left(\mathrm{Cr}^{3^{+}}\right)$in the sapphire, appearing as a sharp doublet at $1021 \mathrm{~cm}^{-1}$ and $1050 \mathrm{~cm}^{-1}$ with red-line excitation. The TNT particle spectra thus illustrate a background interference problem which is excitation dependent but not necessarily thermally induced. 
The chemical composition of South Pole atmospheric aerosol is of interest in many areas atmospheric science, particularly in relation to a better understanding of the global ansport of pollutants in the lower stratosphere and to the long-range effects on the rth's climate. A major thrust of stratospheric aerosol studies has been in the physical d morphological characterization of the aerosol [64,65]. Microchemical spot tests have own sulfate to be a principal component of the aerosol. Results of scanning electron croscopy (SEM), energy-dispersive $x$-ray microanalysis, $x$-ray diffraction, and wet-chemical udies have implicated the existence of $\mathrm{H}_{2} \mathrm{SO}_{4},\left(\mathrm{NH}_{4}\right)_{2} \mathrm{SO}_{4}$, and $\left(\mathrm{NH}_{4}\right)_{2} \mathrm{~S}_{2} \mathrm{O}_{8}$ [65]. As part of larger, joint study with the University of Maryland on the characterization of South Pole rosol, particulate samples from the antarctic atmosphere were collected for micro-Raman vestigation [15]. The samples were obtained during two research expeditions to South Pole ation, Antarctica. The collections utilized gound-based air samplers; both sapphire and thium fluoride substrates were employed with size-segregating impactors. The results of e microprobe analysis give direct evidence for the existence of sulfate species in the uth Pole aerosol. However, further studies are needed to clarify many remaining questions ncerning the in situ nature of the aerosol. Foremost are questions pertaining to sampling fects, the likely reactions of the acid aerosol with other atmospheric constituents, and th the collection substrate, as well as chemical transformations that may have occurred iring sample storage and handling [15].

Most of the measurements were performed on aerosol collected on the two lower impactor ages (size cut-off $\widetilde{<} 4 \mathrm{\mu m}$ ) as the upper three impaction stages bore virtually no iterial. The bulk of the material found on the fourth and fifth stages was of size less Ian $\sim 2-3 \mu \mathrm{m}$ and consisted of discrete microparticles and extensively agglomerated submicroter aerosol. Since the samples were inadvertently exposed to ambient laboratory air and many cases to humidity conditions high enough for moisture absorption to have taken ace, the extreme hygroscopic nature of the aerosol could be noted. In some samples, icroscopic droplets were seen to have formed and to have coalesced, forming larger microoplets. Thus, measurements were made also on particles formed by the recrystallization

such droplets when samples were returned to conditions of low ( 20 percent relative) umidity by flushing the sample compartment of the instrument with dry nitrogen.

SEM/x-ray analysis of all aerosol samples showed sulfur as the only element detected id revealed two different crystalline morphologies. Particles of one distinct morphology are identified with ammonium sulfate and were found on both sapphire and lithium fluoride ubstrates. The second distinct morphology has only been observed for particles sampled on if substrates which on the basis of their spectra were shown to consist of lithium 17 fate.

Figures 13 and 14 show representative spectra obtained in these studies of the South Jle aerosol. The spectrum of figure 13 is from one of the recrystallized particles located n the fourth stage (sapphire substrate) of an impactor sample. Examination of the posiions, intensities and shapes ( $\mathrm{cf}$. Table 1 and fig. 9) of the observed Raman bands ndicates that the microcrystal principally consists of $\left(\mathrm{NH}_{4}\right)_{2} \mathrm{SO}_{4}$ [57]. The bands arising rom $\mathrm{SO}_{4}{ }^{2-}$ vibrations are seen centered at frequencies $452,614,977$, and $1080 \mathrm{~cm}^{-1}$. elative to the band intensities observed for the sulfate vibrations, we do not expect to etect the bands due to the internal vibrations of the $\mathrm{NH}_{4}{ }^{+}$ion because of their comparaively much weaker scattering intensity. These bands have shifts centered at 1429 and $669 \mathrm{~cm}^{-1}$. The two broad bands at $\sim 7350$ and $\sim 7600 \mathrm{~cm}^{-1}$ are the carbon bands discussed efore (cf. figs. 5, 6, and 7) and indicate some amount of a carbonaceous contaminant ossibly adsorbed by the particle during sample handling. The result shown in figure 14 is onsistent with the observations made in the sample analysis discussed above. In this case he aerosol had been collected on a lithium fluoride substrate. The spectrum is from a icroscopic region ( $\sim 6 \mathrm{~m}$ in diameter, defining a sample size estimated to be well under ng) within a dense deposit of profusely aggregated submicrometer particles. A useful pectrum from anywhere in this sample area could only be obtained with $647.1 \mathrm{~nm}$ excitation, $s$ excitation with the $514.5 \mathrm{~nm}$ line produced intolerably high spectral background levels. oth morphological forms were observed in the area analyzed, and the normally smooth and 01 ished substrate surface appeared rough and pitted. Again, the principal bands point to mmonium sulfate as the major component of the aerosol, based on frequency assignments made ith reference to neon calibration lines omitted from the spectrum but indicated by bars elow the recording trace. However, a second component is indicated by the band with Raman 
shift $1008 \mathrm{~cm}^{-1}$, which in this analysis is attributed to the sulfate symmetric stretch in lithium sulfate (presumably $\mathrm{Li}_{2} \mathrm{SO}_{4} \cdot \mathrm{H}_{2} \mathrm{O} ; \mathrm{cf}$. Table 1) [66]. Other bands characterizing $\mathrm{Li}_{2} \mathrm{SO}_{4}$ are much weaker and are not seen owing to the appreciable spectral background. Microprobe analysis of other sample regions showed lithium sulfate to be associated with ammonium sulfate to varying degrees, with some spectra being diagnostic of an essentially pure, hydrated phase of $\mathrm{Li}_{2} \mathrm{SO}_{4}[15]$.

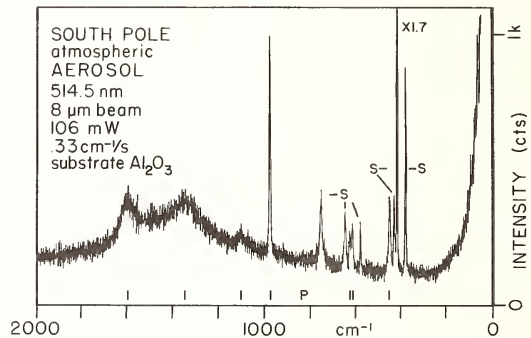

Figure 13. Raman microprobe spectrum of impactor-collected South Pole aerosol, Stage 4. Indicated are excitation wavelength, size (diam.) of laser spot, laser powel (at sample), scan rate, and sample support. Measurement time constant, 1.: sec; spectral slit width, $3 \mathrm{~cm}^{-1}$. Particle analyzed is $\sim 3 \mu \mathrm{m}$ in size. Band labeled $P$ and $S$ are due to the particle and substrate, respectively.

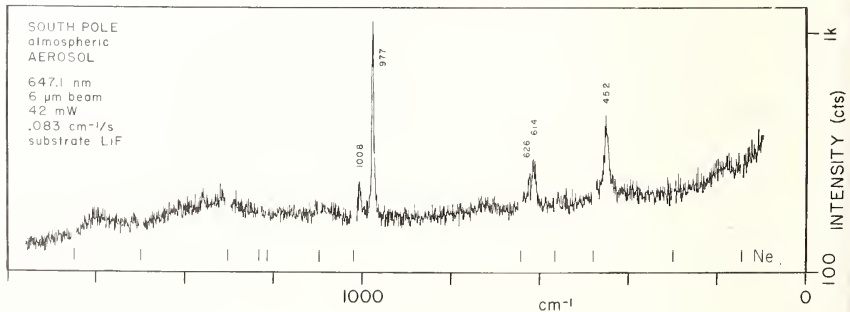

Figure 14. Microprobe spectrum of submicrometer South Pole aerosol collected on Stage 5 Measurement conditions are indicated. Other parameters are: time constant $10.0 \mathrm{sec}$; spectral slit width, $3 \mathrm{~cm}^{-1}$; intensity, 1200 counts full scale. 
From these observations we conclude that a sulfate component (e.g., $\mathrm{H}_{2} \mathrm{SO}_{4}, \mathrm{HSO}_{4}{ }^{-}$) of atmospheric aerosol has undergone a chemical transformation in the sampling process, as $n$ from its reaction with the $\mathrm{LiF}$ substrate resulting in $\mathrm{Li}_{2} \mathrm{SO}_{4}$. This is reasonable, for hium fluoride is a reactive material, whereas sapphire is not. Further evidence of the rosive nature of the sampled aerosol was seen in samples collected on sapphire substrates ring thin-film aluminum (particle finder) grids. For these samples the aluminum film had roded away in all areas where the acid sulfate aerosol had impacted.

We have verified these conclusions by measurements of laboratory-generated aerosol. furic acid aerosol collected on LiF substrates reacts instantaneously forming microstalline material of the same morphological characteristics as are noted in the South e samples. Microprobe measurements show these to be $\mathrm{Li}_{2} \mathrm{SO}_{4}$ microcrystals. When ionium sulfate aerosol is sampled on LiF and stored under ambient laboratory conditions several days, no aerosol-substrate interaction is evident.

Analysis of Fly Ash from Power Plants

\section{Stack Particulates from 0il-Fired Combustion}

To assess the effect of pollutant particles emitted from power plants on man and the ironment, more information is needed on the composition of fly ash. Existing data on the emical properties of fly ash are largely limited to the elemental composition of bulk iples of particulates collected in the stack and in the plume of power plants. Most of at is known about fly ash has been determined from the study of particulate emissions from al-fired plants. In comparison, much less has been learned about the emissions from oilced power plants. In work reported on the analysis of particulates from fuel oil combuson, the emphasis has been on relating the physical/chemical properties of bulk samples of a ash to the elemental composition of the fuels used and the conditions of the combuson. The crude oils burned in power generation exhibit wide variations in sulfur content =/pically $0.5-2.5$ wt.\% S) and in the inorganic trace element composition. Vanadium is ually the major heavy metal (typically 5-400 ppm V) component. Few studies, however, le appeared on the characterization of single particles emitted by oil-fired power ants [67].

We have applied the Raman microprobe to the study of stack aerosols and fly ash rticles from power plants [68]. The most extensive measurements have been made on stackspended particulates from an oil-fired power plant $[13,68]$. Stack aerosol samples were 1 lected by extractive (i.e., out-of-stack) sampling employing five-stage cascade impactors de available to NBS for feasibility studies by the Environmental Protection Agency (EPA). a particular power plant monitored was one burning a fuel of relatively high sulfur (about 5 wt.\% S) and vanadium (approx. $400 \mathrm{ppm} \mathrm{V)} \mathrm{content.} \mathrm{Chemical} \mathrm{analysis} \mathrm{of} \mathrm{the} \mathrm{fuel} \mathrm{also}$ owed trace concentrations of $\mathrm{Ni}(16 \mathrm{ppm})$, Fe $(6 \mathrm{ppm})$, and $\mathrm{Mg}(5 \mathrm{ppm})$. Particle sampling $s$ conducted at a sampling port where the stack temperature was $164{ }^{\circ} \mathrm{C}$. Representative of ese stack aerosol deposits on sapphire substrates is the collection of particles shown in e optical micrograph of figure 15. The field contains the various types of solid particutes found on the fourth impaction stage. Observation in the light microscope shows wide riations in the size and shapes of particles and in optical properties, with microcrystals dendritic morphology showing colors from brown to yellow-green and particles of globular ape exhibiting similar colors in a generally colorless, transparent matrix interspersed th bits of black, opaque material. The majority of the particles on this and on the other ages of the sampler are too large to be accounted for by the size cut-off characteristics each stage. This indicates that much of the material found in these samples has formed the collection surface through the interaction of solid/liquid microparticles with other mponents of the gas/vapor phase of the sampled stack aerosol. 


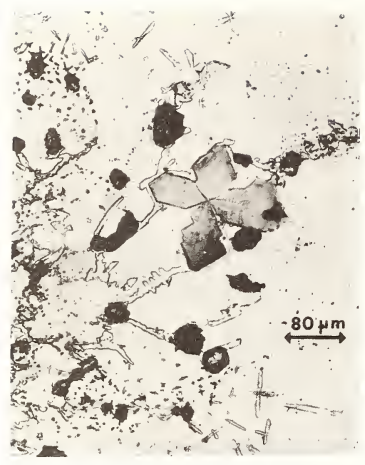

Figure 15. 0ptical micrograph of oil-fired power plant stack particulates collected on a sapphire substrate. Emissions found on the fourth stage of a five-stage impaction sampler.

Typical of the results from particles with dendritic morphology is the spectrum show in figure 16, obtained from probing a small area of the large, four-leaf microcrystal (cf. fig. 15). The predominant features in this and numerous other spectra from particles o this type are bands with Raman shifts of abcut 145, 280, and $1000 \mathrm{~cm}^{-1}$. Taken together these are characteristic of vanadium pentoxide $\left(\mathrm{V}_{2} \mathrm{O}_{5}\right)$ [69]. Qualitative comparison with reference spectrum shows good agreement, except for several medium-intensity bands in thi region 820-970 $\mathrm{cm}^{-1}$ which are absent in the spectrum of pure $\mathrm{V}_{2} \mathrm{O}_{5}$ (assigned frequencies ar, $104,144,285,406,701$, and $\left.995 \mathrm{~cm}^{-1}\right)[13,69]$. These additional bands are due to a secon component, so far unidentified. However, they are absent in the spectra of numerous other brown dendritic microcrystals (of size down to a few micrometers) whose spectra are, in al respects, identical to that of $\mathrm{V}_{2} \mathrm{O}_{5}$.

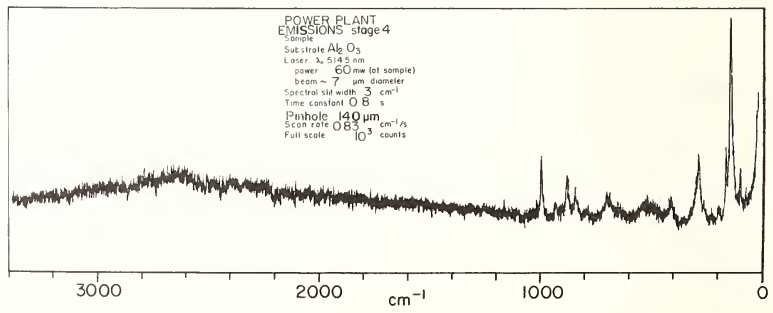

Figure 16. Raman spectrum recorded from probing a small region of the large, four-leave microcrystal seen in the micrograph of figure 15. Measurement conditions use in the analysis are indicated. 
Our finding of $\mathrm{V}_{2} \mathrm{O}_{\mathrm{S}}$ as a principal component of these particulate emissions is consisit with results reported from a study of oil ash particles by Auger microprobe analysis 7]. From the peak height ratios of $V$ and 0 observed in the Auger spectrum, it was conuded that these elements existed in these particles as $\mathrm{V}_{2} \mathrm{O}_{\mathrm{s}}$.

Microparticles of different colors and morphologies have yielded spectra characteristic other compounds. The particles of globular shape (cf. fig. 15) exhibit spectra many of ich indicate the presence of complex crystalline sulfates. (To this point, vanadyl Ifate - $\mathrm{VOSO}_{4}$ - has not been found in these particle collections $\left.[13,69]\right)$. They typically Jw four well-resolved bands, with a strong, sharp band around $985 \mathrm{~cm}^{-1}$, and broader bands lesser intensity centered around 625,460 , and $150 \mathrm{~cm}^{-1}$, the last being a lattice mode. ese features are indicative of crystalline double sulfates, and in particular hydrated tal ammonium sulfates [70]. We suspect that particle transformations have occurred from bosure to ambient ammonia.

In the analysis of stack particles from these impactor samples, our interpretation the spectra invariably must take into consideration the effects of the measurement on e sample. Figure 17 shows a typical result from the analysis of a stack particle for ich this series of consecutive spectra documents measurement-induced sample changes. The $\supset(A)$ and bottom $(C)$ spectra were obtained under identical, low-irradiance conditions; the ddle one (B), at high irradiance. The spectra permit the following interpretation. ckel ammonium sulfate, $\mathrm{NiSO}_{4} \cdot\left(\mathrm{NH}_{4}\right)_{2} \mathrm{SO}_{4} \cdot 6 \mathrm{H}_{2} \mathrm{O}$, is the major - and, in the top spectrum the ly - component of the microparticle identified by comparison with Raman data cited in the terature [70,71] and reference spectra obtained from particles of this salt. The crystalne solid has its strongest band ( $\nu_{1}$ stretching mode) at $985 \mathrm{~cm}^{-1}$ and the other internal 1 fur-oxygen modes at about 455, 625, and $1110 \mathrm{~cm}^{-1}$ (cf. Table 1). The high-irradiance asurement (B) gives evidence of a second (minor) component, with the appearance of a band $144 \mathrm{~cm}^{-1}$, characteristic of $\mathrm{V}_{2} \mathrm{O}_{\mathrm{S}}$. Other bands due to $\mathrm{V}_{2} \mathrm{O}_{\mathrm{s}}$ are obscured by the high minescent background of the spectrum. In parallel measurements on microparticles of two her oxides of vanadium, $\mathrm{V}_{2} \mathrm{O}_{3}$ and $\mathrm{V}_{2} \mathrm{O}_{4}$ (or $\mathrm{VO}_{2}$ ), we have shown that these lower oxides are ansformed by the intense laser radiation (via thermal oxidation) to $\mathrm{V}_{2} \mathrm{O}_{5}$. Both of these wer oxides are dark (black/green) colored and are highly absorbing at $514.5 \mathrm{~nm}$. The ddle spectrum also shows the graphitic carbon bands. The third measurement (C) shows proved signal-to-background resulting from sample changes that affected the presence of minescing (e.g., fluorescing) constituents or contaminants. 


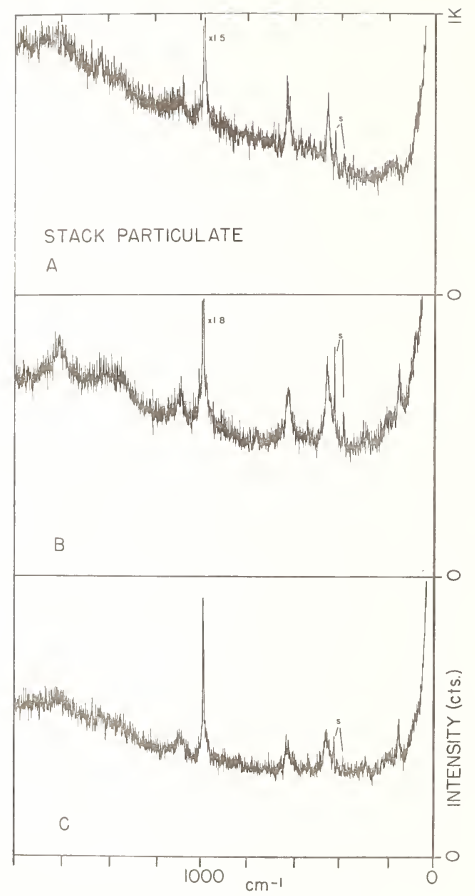

Figure 17.

Spectra of a stack particulate from an oilfired power plant, illustrating laserinduced spectral changes. Analysis conditions: particle size, $8 \times 12 \mu \mathrm{m}$; substrate, $\alpha-\mathrm{Al}_{2} \mathrm{O}_{3} ;$ laser, $\lambda_{0}=514.5 \mathrm{~nm} ;$ spectral slit width, $3 \mathrm{~cm}^{-1}$; intensity, 1000 counts full scale. Parameters varied: power (at sample), $25 \mathrm{~mW}(\mathrm{~A}, \mathrm{C}), 120 \mathrm{~mW}(\mathrm{~B})$; beam spot, $\sim 18 \mu \mathrm{m}$ diameter $(A, C), \sim 8 \mu \mathrm{m}(B)$; time constant, $3.0 \mathrm{sec}(A, C), 1.0 \mathrm{sec}(B)$; scan rate, $20 \mathrm{~cm}^{-1} / \mathrm{min}(\mathrm{A}, \mathrm{C}), 50 \mathrm{~cm}^{-1} / \mathrm{min}$ (B). Bands marked $S$ arise from the scattering by the sapphire substrate.

\section{Coal Fly Ash}

Some results have been obtained in the study of coal fly ash particles, and their spectra have been correlated with microprobe spectra of synthetic glasses of known elemental composition [68]. The bulk of coal fly ash consists of solids of glass-like structure formed by high-temperature processes. The matrix of the ash consists primarily of insoluble alumino-silicate glasses, with the surface layers of ash particles usually showing a predominance of certain trace elements [72]. Our conclusions drawn from a study of these spectra are in agreement with those drawn by others from spectroscopic studies of bulk glasses and various mineral silicates of glass-like structure $[73,74]$. The lack of longrange crystalline order in these materials results in extensive broadening of most spectral features. The vibrational spectrum reveals the type of glass (e.g., high or low silica content) but is much less indicative of the composition of the material. Thus, certain mineral glasses produced by high-temperature reactions may yield well-defined and reasonably intense Raman spectra, whereas others give extremely poor spectra because of the low degree of order in the glass network. It can be generally concluded that the more complex the composition of a glass becomes, the weaker and the more diffuse the Raman bands become. Spectra are then usually flat and featureless; sometimes a sharp band may be identified with included bits of a crystalline phase (e.g., silicate). 
Mineral fibers and particles in the environment have been of increasing concern as a 3) th hazard, particularly the sheet and chain silicates commonly termed asbestos. We ve employed the Raman microprobe to obtain characteristic spectra of some common sheet and ain silicates (anthophyllite, tremolite, and talc are the principal minerals discussed re) and to identify any differentiating features in the spectra that would permit the brous and nonfibrous forms of a given mineral to be distinguished [16].

Raman spectra of both talc plates and fibers (pictured in figure 18) are presented in gure 19. These spectra show essentially identical features and are indistinguishable. ectral investigations of both fibrous and nonfibrous forms of other silicates showed rresponding results. No systematic differences were found in the Raman spectra of the orous and nonfibrous morphologies of a given mineral; however, each mineral has a distinct $\exists$ characteristic Raman spectrum.
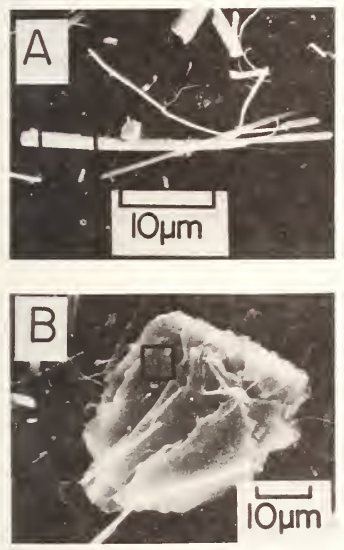

Figure 18.

Scanning electron micrographs of talc particles. Areas of investigation by Raman and electron microprobes are indicated by a square. A: $47.4 \times 1.7 \mu \mathrm{m}$ fiber; magnification, 2800; working distance factor, 0.32 ; elemental composition by electron microprobe - Mg, Si. B: $33.1 \times 23.5 \mu \mathrm{m}$ platy crystal; magnification, 2800 , working distance factor, 0.90 ; elemental composition by electron microprobe $-\mathrm{Mg}, \mathrm{Si}$. 


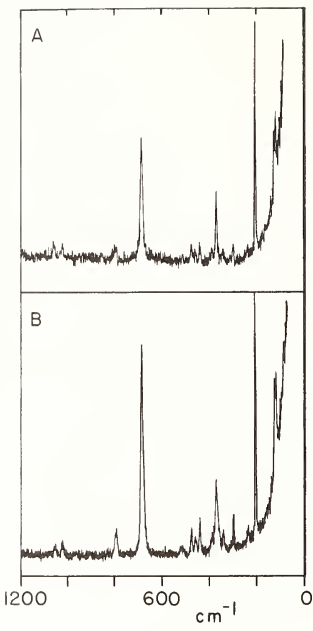

Figure 19. Raman microprobe spectra of fibrous and platy talc. A: $47.4 \times 1.7 \mu \mathrm{m}$ fiber pictured in fig. 18A; substrate, LiF; laser, $\lambda_{0}=514.5 \mathrm{~nm}$; power, $75 \mathrm{~mW}$ (at sample); beam spot, $\sim 8 \mu \mathrm{m}$ diameter; spectral slit width, $3 \mathrm{~cm}^{-1}$; time constant, $2.0 \mathrm{sec}$; scan rate, $20 \mathrm{~cm}^{-1} / \mathrm{min}$; intensity, 1000 counts full scale. B: $33.1 \times 23.5 \mu \mathrm{m}$ platy crystal pictured in fig. 18B; same conditions as for spectrum A except time constant, $6.0 \mathrm{sec}$; scan rate, $10 \mathrm{~cm}^{-1} / \mathrm{min}$.

There are similarities in the spectra which are useful in establishing the type of major structural units and their coordination in the lattice. All the minerals studied have in common a silicate strip formed by crosslinking pyroxene chains. The bands associated with the silicon-oxygen motions are assigned in the regions from 600 to $1200 \mathrm{~cm}^{-1}[75,76]$. A brucite-like strip exists between silicate layers and is composed of octahedrally coordinated cations $\left(\mathrm{Mg}^{2+}\right.$ in talc and tremolite) and thus metal-oxygen vibrational modes are observed in the region between 200 and $450 \mathrm{~cm}^{-1}$ (compare figures $20 \mathrm{~A}$ and $20 \mathrm{C}$ ). Some silicate network deformation modes also extend into this region. The increased complexity of this region as observed in the tremolite spectrum (figure $20 \mathrm{~A}$ ) results from the existence of cations in sites characteristic of amphiboles either of different coordination or nonequivalent to the octahedral sites of the brucite layer. The Raman spectra of microscopic particles of talc and tremolite exhibit sharp, well defined peaks indicating a high degree of site occupancy and structural order. Using these spectra as representative of the ordered structure, we can predict, to some extent, the effects of both a simple cationic substitution as in actinolite (where $\mathrm{Fe}^{2+}$ replaces $\mathrm{Mg}^{2+}$ ) and a major compositional variation and site occupancy disorder as in anthophyllite (figure 20B). In this spectrum, broadening, shifting, and splitting of spectral features are observed in those regions characteristic of the metal-oxygen modes and in the very low frequency, external lattice mode region below $200 \mathrm{~cm}^{-1}$. 


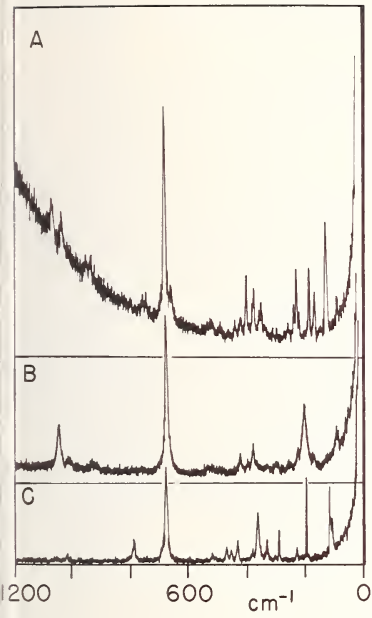

Figure 20 .

Raman spectra of single microparticles of tremolite (A), anthophyllite (B), and talc (C). A: tremolite - particle size, $12 \times 30 \mu \mathrm{m}$; substrate, LiF; laser, $\lambda_{0}=514.5 \mathrm{~nm}$; power, $90 \mathrm{~mW}$ (at sample); beam spot, $\sim 6 \mu \mathrm{m}$ diameter; spectral slit width, 3 $\mathrm{cm}^{-1}$; time constant, $0.5 \mathrm{sec}$; scan rate, $50 \mathrm{~cm}^{-1} /$ min; intensity, 1000 counts full scale. B: anthophyllite - particle size, $10 \times 20 \mu \mathrm{m}$; same conditions as for A except power, $95 \mathrm{~mW}$ (at sample). C: talc - particle size, $5 \times 6 \mu \mathrm{m}$; same conditions as for A except power, $75 \mathrm{~mW}$ (at sample); beam spot, $\sim 8 \mu \mathrm{m}$ diameter; time constant, $1.0 \mathrm{sec}$.

\section{Analysis of Fluid Inclusions in Minerals}

The analysis of fluid inclusions in minerals provides information on many mineralogcal, geological, and geochemical processes. These inclusions consist of microscopically rapped and confined liquid and gaseous phases and frequently coexisting solid phases daughter crystals). Fluid inclusions are abundant in many terrestrial mineral samples and ay be as large as several hundred micrometers.

Raman microprobe techniques have been successfully applied to the non-destructive nalysis of microscopic inclusions in minerals $[9,55,77]$. These studies have included the dentification and characterization of microscopic solid (e.g., $\mathrm{CaSO}_{4}$ ), liquid (e.g., disolved $\mathrm{SO}_{4}{ }^{2-}, \mathrm{CO}_{3}{ }^{2-}, \mathrm{CO}_{2}$ ) and gaseous phases (e.g., $\mathrm{CO}_{2}, \mathrm{H}_{2} \mathrm{~S}, \mathrm{CH}_{4}$ ).

A result from this field of investigation is shown in figure 21 . The sample analyzed s a specimen of natural quartz $\left(\alpha-\mathrm{SiO}_{2}\right)$ in which was localized an irregular inclusion of ize $\sim 68 \mu \mathrm{m}$ (diam.). The upper face of the inclusion was $\sim 18 \mu \mathrm{m}$ below the surface of the uartz host. By examination in the Raman microprobe, the inclusion could be shown to ontain two separate phases (solid and liquid), consisting of several microscopic daughter rystals in the brine within the fluid inclusion. The spectra of figure 21 are representaive of these inclusion studies. The spectrum of the host mineral is characteristic of rystalline quartz $[59,78]$. The liquid phase portion of the inclusion consists of a brine i.e., a salt solution) in contact with several microscopic daughter crystals. The spectrum $f$ the brine shows bands characteristic of dissolved sulfate (cf. Table 1 and fig. 9) 56]. The sharp band at $\sim 981 \mathrm{~cm}^{-1}$ corresponds to the symmetric stretching mode $\left(\nu_{1}\right)$ of $\mathrm{O}_{4}{ }^{2-}$ in aqueous solution. Broader bands centered around $1640 \mathrm{~cm}^{-1}$ and $3400 \mathrm{~cm}^{-1}$ are ssigned to the bending and stretching modes, respectively, of the water in the brine. hese bands are all superimposed upon the spectrum of the host (which represents an intererence). The third spectrum is that of a typical daughter crystal, a platelet only a few icrometers $(<10 \mu \mathrm{m})$ in size. Comparison with a reference spectrum of anhydrite identifies his to be $\mathrm{CaSO}_{4}$. 


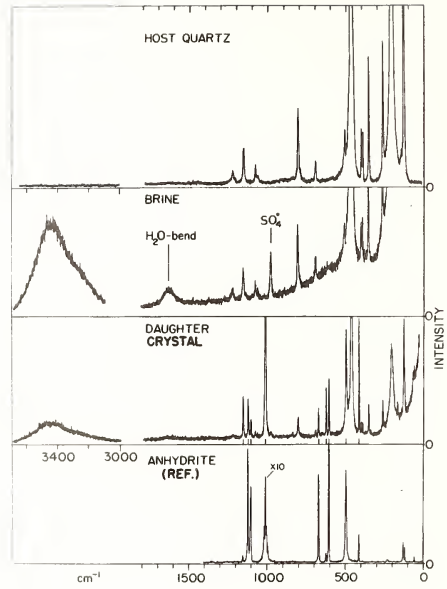

Figure 21.

Spectra recorded in the Raman microprobe analysis of fluid inclusions in a sample of natural quartz. From top to bottom: (i) spectrum of the host $\left(\alpha-\mathrm{SiO}_{2}\right)$; (ii) spectrum of brine inclusion containing dissolved sulfate; (iii) spectrum of daughter crystal $\left(\mathrm{CaSO}_{4}\right)$, size $5 \times 7 \times 4 \mu \mathrm{m}$; (iv) reference spectrum of $\mathrm{CaSO}_{4}$ crystal. Spectra recorded with $514.5 \mathrm{~nm}$ excitation, $80 \mathrm{~mW}$ laser power (at sample), $\sim 6 \mu \mathrm{m}$ diameter beam spot, $3 \mathrm{~cm}^{-1}$ spectral resolution. Raman frequencies of the reference anhydrite are marked along the base line of the spectrum of the daughter crystal.

The concentration of dissolved sulfate in the brine has been estimated to be $12 \mathrm{ppt}$, based on calibrations that suggest a detection limit for dissolved sulfate in such systems of at least 1 ppt.

Often severe limitations to inclusion studies of minerals result from heating of absorbing phases within the inclusion and from spectral interferences by the host [55]. These same considerations apply to the analysis of cellular inclusions (e.g., microscopically localized, bioaccumulated waste products) in biological tissues.

\section{H. Microanalysis of Biological Tissues}

In the study of biological samples of macroscopic size, Raman spectroscopy has evolved as an effective method for elucidating the structure and conformation of biomolecules $[79,80]$. With the advent of Raman microprobe techniques, great possibilities for unique investigations appear to be opening up in biology, pathology, and tissue research to obtain molecular information at the cellular level. To explore these possibilities, the Raman spectra of microsamples of pure biological molecules have been examined, and these studies have been extended to investigations of standard histological sections of biological tissues $[17,18]$. The biological compounds studied in the pure state are "simple" molecules (e.g., urea, cholesterol, amino acids, sugars), nucleotides (e.g., ADP, ATP), and proteins (e.g., bovine serum albumin, collagen). These biomolecules give a richly detailed vibrational spectrum with good sensitivity and are amenable to molecular-level interpretation.

Tissue studies have been made in two areas of application, the characterization of mineralizing tissues [17] and pathological microanalysis [18]. The diagnostically useful spectra obtained from these fragile biological materials were correlated with the information from conventional morphological and $x$-ray microanalytical methods $[40,41]$. Information regarding the precise distribution, relative abundance, and molecular identity of the 
onstituents in such biological matrices is a significant contribution towards understanding any essential tissue and cellular processes.

Initially there was some concern that biological tissues would be quickly burned under he intense irradiation. Under carefully chosen measurement conditions this has not proved 0 be the case, however, and multiple sections of tissue conventionally prepared have been table in the beam so that quite satisfactory spectra could be obtained. In the analysis of oft tissue with $514.5 \mathrm{~nm}$ excitation, laser irradiances typically in the range from 2 to $0 \mathrm{~kW} / \mathrm{cm}^{2}$ have been utilized. In investigations of hard tissue (e.g., bone and tooth), rradiance levels as high as $0.2 \mathrm{MW} / \mathrm{cm}^{2}$ have been safely focused onto the specimen without eleterious effects. Fluorescence interferences have been encountered in some situations e.g., analysis of lung sections) and the origin of this fluorescence can sometimes be raced to sample preparation.

\section{Biological Mineralization}

Hard tissue formation is a complex process consisting of different steps. Although uch research on individual stages of this process has been done, the detailed molecular ransformations of the matrix elements are incompletely understood. Of particular imporance in this research is the non-intrusive examination of the intact tissue and the letermination of the principal components.

In collaboration with workers at the NIH National Institute of Dental Research, the NBS icroprobe was used in the examination of thin (5-10 $\mu \mathrm{m}$ thickness) sections of cartilage, one, and tooth. Of interest in the study of these specimens are the processes leading to .he calcification of tissues and the premineralization of bone. This requires the ability - distinguish - in microscopic regions of the tissue - between organically and inorgancally bound calcium and phosphorus, for example.

Two types of mineralizing tissue have been studied most extensively, the enamel of the ontinuously erupting rat incisor and the embryonic chick tibia [17]. For these types of issue, the initial site and regions of progressive mineralization can be easily located in appropriately prepared cryosections and examined independently.

The major inorganic component of vertebrate hard tissue is an impure (biological) yydroxyapatite, $\mathrm{Ca}_{10}\left(\mathrm{PO}_{4}\right)_{6}(\mathrm{OH})_{2}$, with small amounts (typically $\sim 2-4 \%$ ) of carbonate in the lature tissue. The chief organic component of mineralizing tissues consists of a hydrated roteinaceous matrix, collagenous in the case of bone and dentin and non-collagenous for lineralizing ename 1.

The section of chick tibia shown in the light micrograph of figure 22 is representative of mineralizing tissue samples that have been prepared for Raman microprobe analysis. Good ipectra have been obtained in measurements with spatial resolution typically of 6-15 $\mu \mathrm{m}$ from areas in the mineralized and non-mineralized zones (i.e., cartilage) of the tibia [17]. 

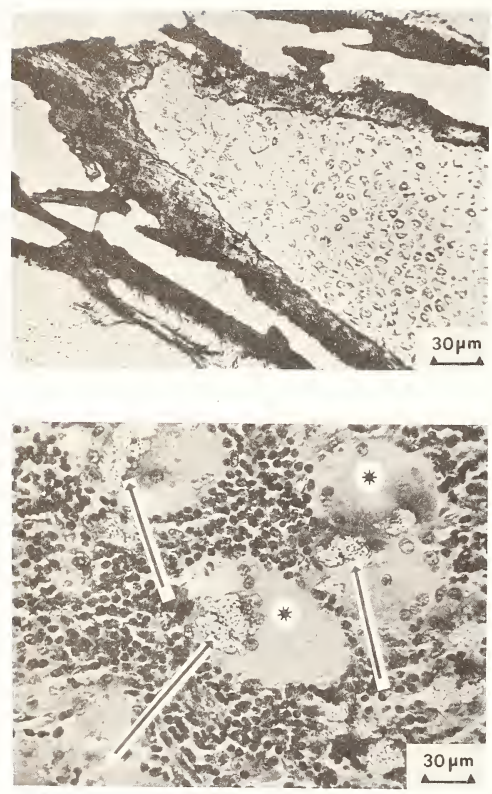

Figure 22.

Photomicrographs, in transmitted light, of thin sections of biological tissues analyzed in the Raman microprobe.

(a) 13-day embryonic chick tibia ( $7 \mu \mathrm{m}$ thickness), air dried. 0paque, dense regions are mineralized zones; lighter regions constitute collagenous tissue matrix. (b) Standard $(5 \mu \mathrm{m})$ section of lymph node with foreign bodies of silicone rubber within multinucleated giant cells. Stained (hematoxylin-eosin). Arrows indicate several typical inclusions of silicone polymer. Asterisk shows cytoplasmic area analyzed to obtain the spectrum of the host tissue matrix.

The spectra of figure 23 were obtained from sequential measurements across a region in rat incisor enamel and indicate the progressive mineralization (from a through d) of the tissue. The spectra are composed of the features characteristic of hydroxyapatite $[45,81]$ superimposed on the spectrum of collagen [82]. The four fundamental modes specific of the internal vibrations of the orthophosphate ion $\left(\mathrm{PO}_{4}{ }^{3-}\right)$ in the apatite lattice are the strong $v_{1}$ (P-0 symmetric stretch) at $962 \mathrm{~cm}^{-1}$, the $v_{2}\left(0-\mathrm{P}-0\right.$ symmetric bend) between $400-500 \mathrm{~cm}^{-1}$, the $v_{3}$ (P-0 asymmetric stretch) between $1000-1100 \mathrm{~cm}^{-1}$, and the $v_{4}(0-P-0$ asymmetric bend) in the region 560-620 $\mathrm{cm}^{-1}$. The $\sim 960 \mathrm{~cm}^{-1}$ band is the most prominent and is a sensitive indicator to changes in the extent of mineralization. The spectra of figure 23 illustrate the dramatic increase in the intensity (approx. eight-fold) of the $\sim 960 \mathrm{~cm}^{-1}$ band from an area (a) in the mineralizing front to a point at the dentino-enamel junction (d). 


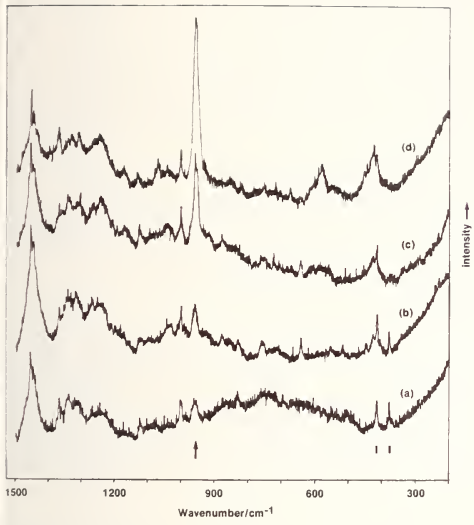

Figure 23 .

Sequential Raman microprobe spectra obtained from microscopic regions $(\sim 7 \mu \mathrm{m}$ apart) in air dried section (10 $\mathrm{mm}$ thickness) of mineralizing rat incisor enamel. Tissue area analyzed extends from the mineralizing front (a) into the dentinoenamel junction (d), a distance of $\sim 30 \mu \mathrm{m}$. Measurement conditions: laser, $\lambda_{0}=514.5$ nm; power, $20 \mathrm{~mW}$ (at sample); beam spot, $7 \mu \mathrm{m}$ diameter; spectral slit width, $3 \mathrm{~cm}^{-1}$; time constant, $15 \mathrm{sec}$; scan rate, $5 \mathrm{~cm}^{-1} / \mathrm{min}$. Tissue section supported by a sapphire substrate.

These studies on mineralizing tissues are continuing. Our efforts are aimed at a more complete understanding of the compositional and structural information obtained from the spectra, with the hope that some insight can be gained regarding any changes in protein structure and conformation during the development of biological mineralization.

\section{Diagnostic Pathology}

Thin sections (5-10 $\mu \mathrm{m}$ thickness) of human biopsy tissue have come under study for the suspected presence of microscopic foreign bodies of silicone polymer [18]. These had been previously examined by pathologists at the University of California, San Diego, using conventional methods. In one case study, the biopsy tissue was one from a patient with axillary lymph node complications which were suspected to be related to an implanted silicone rubber finger joint prosthesis. Standard histologic sections ( $5 \mu \mathrm{m}$; embedded in paraffin) of this biopsy material had been prepared for initial examination by light microscopy, scanning electron microscopy (SEM), and x-ray microanalysis. 0ther parallel sections (unstained) of the lymph node were mounted on sapphire substrates and deparaffinized with xylene for subsequent Raman microprobe analysis. Microscopically, the tissue showed enlarged lymphoid follicles and many multinucleated foreign body giant cells, some of which contained irregular pale-yellow fragments of a translucent material 5-60 $\mu \mathrm{m}$ in diameter. A light micrograph of a stained section of the lymph node, in figure 23 , shows this foreign material within giant cells. SEM/x-ray analysis had demonstrated that these foreign bodies contained silicon. This suggested that the microscopic fragments were, in fact, silicone rubber particles from the prosthesis, but direct molecular identification was not possible from the information furnished by the $x$-ray spectrum. When the tissue section on the sapphire substrate was viewed in the microscope of the Raman microprobe the histology was observed even in an unstained section. Spectra were obtained from various parts of the tissue, and these are shown in figure 24. Measurements were made on areas of the tissue (i.e., the cytoplasm matrix or host) away from the inclusions of foreign material. Spectra were then obtained from a dozen individual inclusions, which showed identical and characteristic peaks at several frequencies. These were compared with spectra obtained under the same conditions from particles abraded directly from a new silicone rubber joint prosthesis. The inclusions in the giant cells gave spectra identical to that from the prothesis material with additional peaks attributable to the tissue matrix. The micro-Raman spectrum 191 
of the prosthesis material is identical to published bulk Raman spectra of polydimethylsiloxane polymers (silicone rubber and gel) [83].

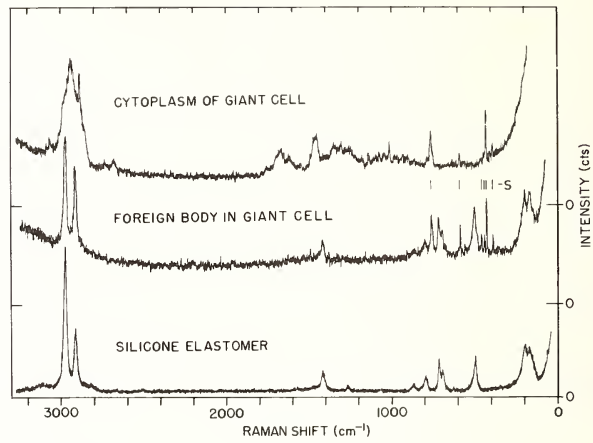

Figure 24. Spectra recorded in the Raman microprobe analysis of a deparaffinized standard $5 \mu \mathrm{m}$ section of lymph node (cf. fig. 22), mounted on a sapphire $\left(\alpha-\mathrm{Al}_{2} \mathrm{O}_{3}\right.$ ) substrate. Measurement parameters common to each spectrum: laser, $\lambda_{0}=514.5$ $\mathrm{nm}$; beam spot, $16 \mu \mathrm{m}$ diameter; exit pinhole, $140 \mu \mathrm{m}$; spectral slit width, $3 \mathrm{~cm}^{-1}$. Top: spectrum of the cytoplasm away from foreign bodies; power, $40 \mathrm{~mW}$ (at sample); time constant, $5.0 \mathrm{sec}$; scan rate, $20 \mathrm{~cm}^{-1} / \mathrm{min}$. Middle: spectrum of a foreign body (size, $\sim 24 \mu \mathrm{m}$ ) located within a giant cell. Measurement conditions as for top spectrum. Bottom: spectrum of a small $(\sim 60 \mu \mathrm{m})$ particle of silicone elastomer from a joint prosthesis. Conditions as for top spectrum, except - power, $60 \mathrm{~mW}$ (at sample); time constant, $0.5 \mathrm{sec}$; scan rate, 100 $\mathrm{cm}^{-1} / \mathrm{min}$. Bands marked $\mathrm{S}$ on the upper and middle spectra are contributed by the sapphire substrate.

This study illustrates the effective potentialities of micro-Raman spectroscopy for the examination - in situ, at the cellular level - of inhomogeneous biological material. Thus, in biological and biomedical work, Raman microprobe analysis can be regarded as an important extension of the well established techniques of fluorescence microscopy and microspectrofluorometry which are commonly employed in the study of intact living tissues $[84,85]$.

\section{Conclusions}

In describing the application of the Raman microprobe to several areas of particle microanalysis, we have given an overview of the kinds of problems that are amenable to investigation by micro-Raman spectroscopy. The results obtained indicate both the unique capabilities of the technique as well as its inherent limitations as an analytical tool. We perceive as one of the strengths of the technique the fact that it is emerging as a valuable complement to the more traditional microprobe methods of elemental analysis.

At this point in the development of Raman microprobe analysis, entirely new information can be obtained from particles or microscopic sampling regions approaching one micrometer in dimensions. Molecular microprobing in the submicrometer domain is not routinely feasible with instrumentation now in use. 
Various factors determine the outcome of a Raman microprobe measurement and the amount $f$ useful analytical information obtained from the sample. The important variables appear 10 be the molecular nature of the species itself, its concentration and distribution in the ample and the chemical and physical complexity of the matrix in which the scattering olecules reside. From current understanding of these factors, no firm conclusions can be rawn regarding the ultimate limits of detection imposed by either sample size and morphoogy, sample heating and fluorescence, the identity of the scattering molecules, concentraion effects, and the compositional complexity of the sample. These questions have been ddressed only partially and their further elucidation must await future studies.

In many programs of materials characterization, the use of Raman microprobe techniques an be expected to yield new and analytically useful information. With the steadily xpanding range of demonstrated applications and advances in experimentation and theory, he future of Raman microprobe analys is looks promising indeed.

We are grateful to acknowledge partial financial support of this work by several igencies of the U. S. Government outside of NBS. These are: Air Force Technical Applicaions Center, AFTAC (microprobe development and applications); Environmental Protection igency, EPA (stationary source emissions and trace organic analysis); National Institute of lental Research, NIDR (biological tissue mineralization); and National Science Foundation, ISF (South Pole aerosol studies). Finaliy, we thank Kurt F. J. Heinrich of the Microanalysis Group at NBS for his guidance and numerous helpful discussions in the course of this research.

\section{References}

[1] Long, D. A., Raman Spectroscopy, McGraw-Hi11, Inc., London (1977).

[2] Hendra, P. J., Laser Raman Spectroscopy, in Vibrational Spectra and Structure, Vol. 2, Durig, J. R. (ed.), Marcel Dekker, Inc., New York, Chap. $2,135-38 \overline{3(1975) .}$

[3] Irish, D. E., and Chen, H., The Application of Raman Spectroscopy to Chemical Analysis, Appl. Spectrosc. $25,1-6$ (1971).

[4] Delhaye, M., and Dhamelincourt, P., Raman Microprobe and Microscope with Laser Excitation, J. Raman Spectrosc. $\underline{3}, 33-43$ (1975).

[5] Rosasco, G. J., Etz, E. S., and Cassatt, W. A., The Analysis of Discrete Fine Particles by Raman Spectroscopy, App1. Spectrosc. 29, 396-404 (1975).

[6] Rosasco, G. J., and Etz, E. S., The Raman Microprobe, A New Analyticai Tool, Res. \& Devel. 28, 20-35 (June 1977).

[7] Dhamelincourt, P., Wallart, F., Leclercq, M., N'Guyen, A. T., and Landon, D. 0., Laser Raman Molecular Microprobe (MOLE), Anal. Chem. 51, 414A-421A (1979).

[8] Etz, E. S., Raman Microprobe Analysis, Principles and Applications, in Scanning Electron Microscopy, 1979, I, SEM, Inc. , 67-82 (1979).

[9] Rosasco, G. J., Microanalysis by Raman Spectroscopy, in Proc. of the Sixth International Conf. on Raman Spectroscopy, Schmid, E. D., Krishnan, R. S., Kiefer, W., and Schrotter, H. W. (eds.), Heyden \& Son Ltd., London, 389-398 (1978).

[10] Etz, E. S., and Blaha, J. J., Investigations into the Critical Measurement Aspects of Raman Microprobe Analysis, in Proc. 14th Microbeam Analys is Society Conf. (1979).

[11] Dhamelincourt, P., Developments and Applications of the Laser-Raman Microprobe MOLE, in Proc. 14th Microbeam Analysis Society Conf. (1979). 
[12] Adar, F., Application of the MOLE to Identification of Molecular Impurities and to Structural Characterization of Microscopic Samples in Situ, in Scanning Electron Microscopy/1979/I, SEM, Inc. (1979).

[13] Etz. E. S., Rosasco, G. J., and Blaha, J. J., Observation of the Raman Effect from Small Single Particles, in Environmental Pollutants: Detection and Measurement, Toribara, T. Y., Coleman, J. R., Dahneke, B. E., and Feldman, I. (eds.), Plenum Publishing Corp., New York, 413-456 (1978).

[14] Blaha, J. J., Rosasco, G. J., and Etz, E. S., Raman Microprobe Characterization of Residual Carbonaceous Material Associated with Urban Airborne Particulates, Appl. Spectrosc. 32, 292-297 (1978).

[15] Cunningham, W. C., Etz, E. S., and Zoller, W. H., Raman Microprobe Characterization of South Pole Aerosol, in Proc. 14th Microbeam Analysis Society Conf. (1979).

[16] Blaha, J. J., and Rosasco, G. J., Raman Microprobe Spectra of Individual Microcrystals and Fibers of Talc, Tremolite, and Related Silicate Minerals, Anal. Chem. $\underline{50}$, 892-896 (1978).

[17] Casciani, F. S., Etz, E. S., Newbury, D. E., and Doty, S. B., Raman Microprobe Studies of Mineralized Tissues: Enamel of the Rat Incisor and the Embryonic Chick Tibia, in Scanning Electron Microscopy/1979/I, SEM, Inc. (1979).

[18] Abraham, J. L., and Etz, E. S., Molecular Microanalysis of Pathological Specimens in Situ with a Laser-Raman Microprobe, to appear in Science (1979).

[19] Kerker, M., Some Recent Reflections on Light Scattering, J. Colloid Interface Sci. $\underline{58}$, $100-112$ (1977).

[20] Benner, R. E., Dornhaus, R., Long, M. B., and Chang, R. K., Inelastic Light Scattering from a Distribution of Microparticles, in Proc. 14th Microbeam Analysis Society Conf. (1979).

[21] Hirschfeld, T., Design Considerations in a Micro-Raman Spectrometer, in Proc. 14th Microbeam Analysis Society Conf. (1979).

[22] Landon, D. 0., The Development of Instrumentation for Microparticle Analysis by Raman Spectroscopy, in Proc. 14th Microbeam Analysis Society Conf. (1979).

[23] Rosasco, G. J., Raman Microprobe Spectroscopy, chapter to appear in Advances in Infrared and Raman Spectroscopy, Vol. 7, Clark, R. J. H., and Hester, R. E. (eds.), Heyden \& Son Ltd., London (1980).

[24] Malissa, H. (ed.), Analysis of Airborne Particles by Physical Methods, CRC Press, Inc., West Palm Beach, Florida (1978).

[25] Koningstein, J. A., Introduction to the Theory of the Raman Effect, D. Reidel Publishing Company, Dortrecht, Holland (1972).

[26] Hathaway, C. E., Raman Instrumentation and Techniques, Chapter 4, in The Raman Effect, Vol. I, Anderson A. (ed.), Marcel Dekker, Inc., New York, 183-285 (1971).

[27] Omenetto, N. (ed.), Analytical Laser Spectroscopy, Vol. 50 in Chemical Analysis, Elving, P. J., Winefordner, J. D., and Kolthoff, I. M. (eds.), Wiley-Interscience, New York (1979).

[28] Talmi, Y., TV-Type Multichannel Detectors, Anal. Chem. 47, 658A and 697A (1975).

[29] Talmi, Y., Baker, D. C., Jadamec, J. R., and Saner, W. A., Fluorescence Spectrometry with Optoelectronic Image Detectors, Anal. Chem. 50, 936A-952A (1978). 
30] Horlick, G., Characteristics of Photodiode Arrays for Spectrochemical Measurements, App 1. Spectrosc. 30, 113-123 (1976).

31] Cooney, R. P., Boutilier, G. D., and Winefordner, J. D., Comparison of Image Devices vs. Photomultiplier Detectors in Atomic and Molecular Luminescence Spectrometry via Signal-to-Noise Ratio Calculations, Anal. Chem. 49, 1048-1057 (1977).

32] Mathies, R., and Yu, N.-T., Raman Spectroscopy with Intensified Vidicon Detectors: A Study of Intact Bovine Lens Proteins, J. Raman Spectrosc. 7, 349-352 (1978).

33] Arthur, J. W., and Lockwood, D. L., Automation Techniques for Raman Spectroscopy, J. Raman Spectrosc. 2 , 53-69 (1974).

34] Downey, J. R., Jr., and Janz, G. J., Digital Methods in Raman Spectroscopy, Chapter 1 in Advances in Infrared and Raman Spectroscopy, Vol. 1, Clark, R. J. H., and Hester, R. E. (eds.), Heyden \& Son Ltd., London, 1-34 (1975).

35] Edge11, W. G., Schmidlin, E., Kuriakose, T. J., and Lurix, P., A Computer-Spectrometer Interactive System for Raman Spectroscopy, Appl. Spectrosc. 30, 428-439 (1976).

36] Etz, E. S., Rosasco, G. J., and Cunningham, W. C., The Chemical Identification of Airborne Particles by Laser Raman Spectroscopy, in Environmental Analysis, Ewing, G. W. , (ed. ), Academic Press, New York, 295-340 (1977).

37] McCrone, W. C., and Delly, J. G., The Particle Atlas, Edition 2, Ann Arbor Science Publishers, Inc., Ann Arbor (1973).

38] Liu, B. Y. H. (ed.), Fine Particles: Aerosol Generation Measurement, Sampling and Analysis, Academic Press, New York (1976).

39] Tanner, R. L., and Newman, L., The Analysis of Airborne Sulfate: A Critical Review, J. Air Pol1. Control Assoc. 26, 737-747 (1976).

40] Hall, T., Echlin, P., and Kaufmann, R. (eds.), Microprobe Analysis as Applied to Cells and Tissues, Academic Press, Inc., (London) Ltd., London (1974).

-41] Abraham, J. L., Documentation of Environmental Particulates in Humans Using SEM and EDXA, in Scanning Electron Microscopy/1979/I, SEM, Inc. (1979).

[42] Keramidas, V. G., and White, W. B., Raman Spectra of 0xides with the Fluorite Structure, J. Chem. Phys. 59, 1561-1562 (1973).

[43] Porto, S. P. S., and Krishnan, R. S., Raman Effect of Corundum, ‥ Chem. Phys. $\underline{47}$, 1009-1012 (1967).

[44] Bennett, H. S., and Rosasco, G. J., Heating Microscopic Particles with Laser Beams, J. Appl. Phys. $\underline{49}, 640-647$ (1978).

[45] Griffith, W. P., Raman Studies on Rock-Forming Minerals, Part II, $\underline{J}$. Chem. Soc. $\underline{(A)}$, 286-291 (1970).

[46] Tuinstra, F., and Koenig, J. L., Raman Spectrum of Graphite, J. Chem. Phys. $\underline{53}$, 11261130 (1970).

[47] Rosen, H., and Novakov, T., Identification of Primary Particulate Carbon and Sulfate Species by Raman Spectroscopy, Atmos. Environ. 12, 923-927 (1978).

[48] Etz, E. S., Wise, S. A., and Heinrich, K. F. J., On the Analytical Potential of MicroRaman Spectroscopy in the Trace Characterization of Polynuclear Aromatic Hydrocarbons, in Trace Organic Analysis: A New Frontier in Analytical Chemistry, Hertz, H. S., and Chester, S. N. (eds.), Proc. 9th Materials Research Symp., NBS Special Publication 519, U. S. Gov't. Printing Office, Washington, D.C. 723-729 (Apri1 1979). 
[49] Kerker, M., McNulty, P. J., Sculley, M. , Chew, H., and Cooke, D. D., Raman and Fluorescent Scattering by Molecules Embedded in Small Particles, J. Opt. Soc. Am. 68, 16761689 (1978).

[50] Kerker, M., and Druger, S. D., Raman and Fluorescent Scattering by Molecules Embedded in Spheres with Radii up to Several Multiples of the Wavelength, Appl. Opt. 18, 11721179 (1979).

[51] Bennett, H. S., and Rosasco, G. J., Resonances in the Efficiency Factors for Absorption: Mie Scattering Theory, Appl. Opt. 17, 491-493 (1978).

[52] Rosasco, G. J., and Bennett, H. S., Internal Field Resonance Structure: Implications for 0ptical Absorption and Scattering by Microscopic Particles, J. 0pt. Soc. Am. $\underline{68}$, 1242-1250 (1978).

[53] Chylek, P., Kieh1, J. T., and Ko, M. K. W., Narrow Resonance Structure in the Mie Scattering Characteristics, Appl. 0pt. 17, 3019-3021 (1978).

[54] Blaha, J. J., Etz, E. S., and Cunningham, W. C., Molecular Analysis of Microscopic Samples with a Raman Microprobe: Applications to Particle Characterization, in Scanning Electron Microscopy/1979/I, SEM, Inc. 93-102 (1979).

[55] Rosasco, G. J., and Roedder, E., Application of a New Laser-Excited Raman Spectrometer to Nondestructive Analysis of Sulfate and other Ions in Individual Phases in Fluid Inclusions in Minerals, submitted to Geochim. Cosmochim. Acta (1979).

[56] Irish, D. E., and Chen, H., Equilibria and Proton Transfer in the Bisulfate-Sulfate System, J. Phys. Chem. 74, 3796-3801 (1970).

[57] Venkateshwarlu, P., Bist, H. D., and Jain, Y. S., Laser-Excited Raman Spectrum of Ammonium Sulfate Single Crystal, J. Raman Spectrosc. 3, 143-151 (1975).

[58] Ananthanarayanan, V., A Comparative Study of the Raman Spectra of Anhydrous Sulfates,

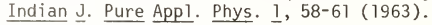

[59] Ross, S. D., Inorganic Infrared and Raman Spectra, McGraw-Hill Book Company (UK) Limited, Maidenhead, Birkshire, England (1972).

[60] Krishnamurthy, N. and Soots, V., Raman Spectrum of Gypsum, Can. J. Phys. 49, 885-896 (1971).

[61] Porto, S. P. S., Giordmaine, J. A., and Damen, T. C., Depolarization of Raman Scattering in Calcite, Phys. Rev. 147, 608-611 (1966).

[62] Ketseridis, G., Hahn, J., Jaenicke, R., and Junge, C., The Organic Constituents of Atmospheric Particulate Matter, Atmos. Environ. 10, 603-610 (1976).

[63] Dollish, F. R., Fately, W. G., and Bentley, F. F., Characteristic Raman Frequencies of Organic Compounds, John Wiley \& Sons, Inc., New York (1974).

[64] Cadle, R. D., The Measurement of Airborne Particles, John Wiley \& Sons, Inc., New York (1975).

[65] Bigg, E. K., Stratospheric Particles, J. Atmos. Sci. 32, 910-917 (1975).

[66] Vassas-Dubuisson, C., Vibrational Spectrum of a Single Crystal of Lithium Sulfate Monohydrate, Compt. Rend. 233, 374-376 (1951).

[67] Cheng, R. J., Mohnen, V. A., Shen, T. T., Current, M., and Hudson, J. B., Characterization of Particulates from Power Plants, J. Air Poll. Control Assoc. 26, 787-790 (1976).

[68] Etz, E. S., Rosasco, G. J., and Heinrich, K. F. J., Chemical And?ysis of Stationary Source Particulate Pollutants by Micro-Raman Spectroscopy, EPA Report EPA-600/2-78-193 (August 1978). 
69] Gilson, T. R., Bizri, 0. F., and Cheetham, N., Single-Crystal Raman and Infrared Spectra of Vanadium (V) Oxide, J. Chem. Soc. (Dalton), 291-294 (1973).

70] Ananthanarayanan, V., Raman Spectra of Crystalline Double Sulfates: Part II. Ammonium Double Sulfates, $\underline{\text { Z. Physik } 166}$, 318-327 (1962).

71] Jain, Y. S. and Bist, H. D., Optical Phonons in $\alpha-\mathrm{NiSO}_{4} \cdot 6 \mathrm{H}_{2} \mathrm{O}$ Single Crystal, J. Raman Spectrosc. 2, 327-339 (1974).

72] Keyser, T. R., Natusch, D. F. S., Evans, C. A., Jr., and Linton, R. W., Characterizing the Surfaces of Environmental Particles, Environ. Sci. Technol. 12, 768-773 (1978).

73] White, W. B., Structural Interpretation of Lunar and Terrestrial Minerals by Raman Spectroscopy, in Infrared and Raman Spectroscopy of Lunar and Terrestrial Minerals,

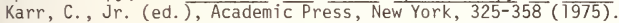

74] Brawer, S. A., and White, W. B., Raman Spectroscopic Investigation of the Structure of Silicate Glasses. I. The Binary Alkali Silicates, $\underline{J}$. Chem. Phys. $\underline{63}, 2421-2432$ (1975).

75] Lazarev. A. N., Vibrational Spectra and Structure in Silicates, Consultants Bureau, New York (1972).

76] Farmer, V. C. (ed.), The Infrared Spectra of Minerals, Mineralogical Society, London (1974).

77] Dhamelincourt P., and Schubnel, H. J., La Microsonde Moleculaire a Laser et son Application a la Mineralogie et la Gemmologie, Rev. Gemm. A. F. G. $\underline{52}, 11-14$ (September 1977).

78] Scott, J. F., and Porto, S. P. S., Longitudinal and Transverse Optical Lattice Vibrations in Quartz, Phys. Rev. 161, 903-910 (1967).

79] Yu, N.-T., Raman Spectroscopy: A Conformational Probe in Biochemistry, CRC Crit. Revs. Biochem. 4, 229-280 (1977).

80] Lord, R. C., Strategy and Tactics in the Raman Spectroscopy of Biomolecules, Appl. Spectrosc. 31, 187-194 (1977).

81] Blakeslee, K. C., and Condrate, R. A., Vibrational Spectra of Hydrothermally Prepared Hydroxyapatites, $\underline{\text { J. Amer. }}$. Cer. Soc. 54, 559-563 (1971).

82] Frushour, B. G., and Koenig, J. L., Raman Scattering of Collagen, Gelatin and Elastin, Biopolymers $14,379-391$ (1975).

83] Maxfield, J., and Shephard I. W., Investigation of Molecular Isomerism in Polydimethylsiloxane by Raman Scattering, Chem. Phys. 2, 433-444 (1973).

84] Parker, C. A., Spectrophosphorimeter Microscopy: An Extension of Fluorescence Microscopy, Analyst 94, 167-176 (1969).

85] Mayer, R. T., and Novacek, V. M. , A Direct Recording Corrected Microspectrofluorometer, J. Microsc. 102 (Pt. 2), 165-177 (1974). 
lational Bureau of Standards Special Publication 533. This Publication Originated from a pecial Session on Particle Analysis, 13th Annual Conference of the Microbeam Analysis ociety, Ann Arbor, MI, June 22, 1978. (Issued April 1980)

\title{
LASER MICROPROBE MASS ANALYSIS (LAMMA) IN PARTICLE ANALYSIS
}

\author{
R. Kaufmann
}

Physiologisches Institut, Lehrstuhl für Klinische Physiologie Universität Düsseldorf, Moorenstr. 5, 4000 Düsseldorf

and

\section{P. Wieser}

Institut für Physik, Universität Hohenheim, Garbenstr. 30 7000 Stuttgart 70

Federal Republic of Germany

Abstract

A laser microprobe mass analyzer (LAMMA) has recently been introduced as an instrument for microprobe analysis of atomic or molecular constituents in organic or inorganic matter. The present contribution gives a short technical description of the LAMMA principle, discusses its performances and limitations with respect to particle analysis (as far as the preliminary results allow) and demonstrates examples of recent studies performed with the LAMMA instrument in various types of particles.

\section{Introduction}

For the chemical analysis of microparticulate material, basically all conventional ethods are applicable as long as one is interested in the average composition of these aterials only. If it comes to the question of differences between single particles, more ophisticated approaches are required. Recently the various techniques of microprobe nalysis became more and more important in studying the chemistry of single particles. hey have already attributed a great deal to our understanding of the mechanisms underlying he forming, aging, and removal of aerosols, or to the significance of specific sorts of articles in pathology or ecological systems.

However, the microprobe techniques available are still far from satisfying the various nalytical request of scientists working in aerosol research or other related fields. The deal instrument should have the following features: (1) Besides an appropriate spatial esolution, an instrument applied in particle analysis should give as much analytical nformation as possible about the atomic as well as about the molecular constituents including isotope discrimination) of a particle; (2) sensitivity should be high enough to etect trace contaminants possibly down to the sub ppmw range; (3) analysis should be uantitative or at least semiquantitative; (4) the measuring procedure and data processing hould be fast enough to accumulate the analytical data of larger particle populations in a easonable period of time; and (5), last but not least, viewing should provide for as much $s$ possible information on the morphology of the particles under investigation.

Clearly, such an instrument does not yet exist. Instead, a variety of techniques are vailable, each of them complying with only one or the other of the above requirements. e will subsequently discuss a new technique which we call laser microprobe mass analysis LAMMA). This technique has recently become available and, according to preliminary tudies, gives promise of fulfilling part of the above requirements where other techniques annot compete. 
A detailed technical description of the instrument has been given elsewhere $[1,2,3]^{1}$. The LAMMA principle is based on laser-induced-mass spectrometry (LIMS). The beam of a pulsed laser ( $t \sim 10 \mathrm{~ns}$ ) is focused through a high power optical microscope onto the target to be analyzed (figure 1). For most applications, the light of a neodymium yttriumaluminum garnet laser in the near or far UV (frequency tripled $=353 \mathrm{~nm}$, or quadrupled $=$ $265 \mathrm{~nm})$ have proven to be optimal. The same microscope is used for viewing the specimen which is preferably a thin section $(\sim 0.3 \mu \mathrm{m})$ or small particles supported by a very thin $(\sim 500 \AA)$ supporting film. No special treatment of the specimen (coating) is necessary.

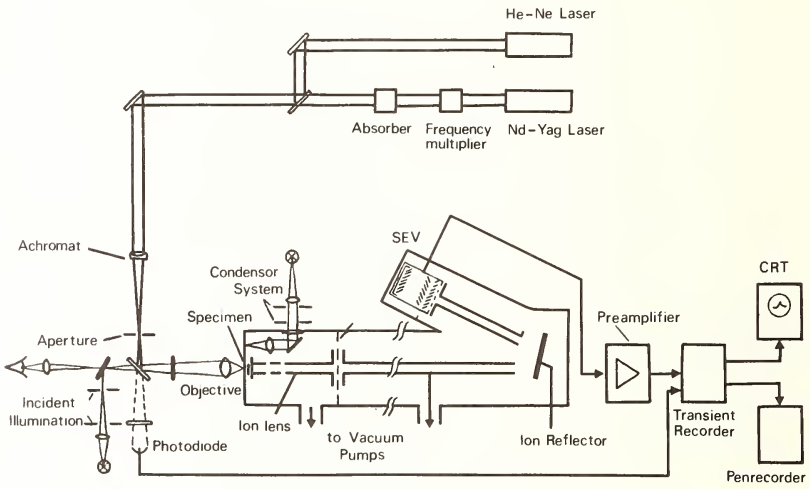

Figure 1. Schematic drawing of the instrumental setup.

Spatial resolution (both optical and analytical) is limited by diffraction to about $0.5 \mu \mathrm{m}$ though much smaller particles can be spotted and analyzed. The specimen is mounted in a vacuum chamber right underneath a thin quartz-window next to the focusing objective. A time of flight (TOF) mass spectrometer is used for the analysis of ions formed in the evaporated sample volume (typically $10^{-12}-10^{-14} \mathrm{~cm}^{3}$ ). Ions are accelerated to an energy of 3-5 keV and focused down to a field-free drift tube onto a detector (open secondary electron multiplier). Ions of different $\mathrm{m} / \mathrm{e}$ ratio are distinguished according to their different flight time which varies with $\sqrt{\mathrm{m}}$ for singly charged ions. As the ions are generated in a very short burst within a volume small enough to constitute essentially a point source, an overall transmission of the spectrometer of $10-50$ percent is achieved. In the instrument so far used for particle analysis, targets are limited to a maximum diameter of some $10 \mu \mathrm{m}$, because optical and ion systems are arranged on opposite sides of the target. Larger particles can be analyzed in grazing incidence with some loss in quantitation.

The mass resolution of the TOF mass spectrometer is limited by the width of the initial energy distribution of the ions, leading to a corresponding spread in flight times. This dispersion is partially compensated for by an ion reflector at the end of the drift tube. The achieved mass resolution is $\mathrm{m} / \Delta \mathrm{m} \sim 800$. Thus, with every laser shot, complete mass

${ }^{1}$ Figures in brackets indicate the literature references at the end of this paper. 
bectra are available in which mass signals differing by one mass number are discriminated , to mass number 1000 (see also figure 2). The electron multiplier output signal is nplified, digitized with sampling intervals of $10 \mathrm{~ns}$ and stored for slow readout or further ta processing by an on-line computer.
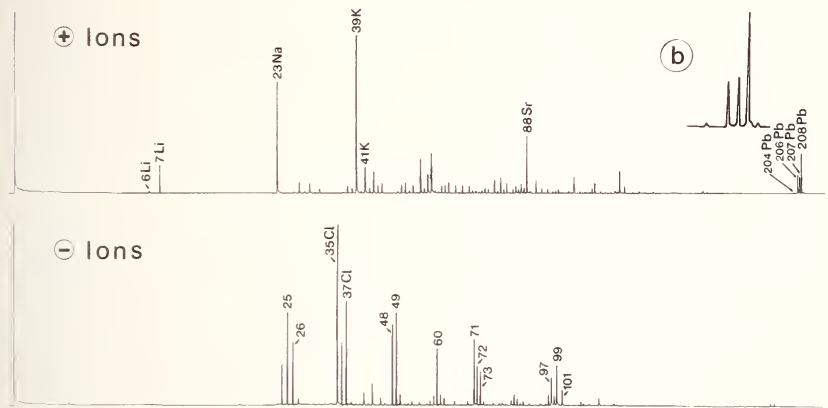

igure 2. Typical LAMMA spectra of positive and negative ions obtained from an epoxy resin standard specimen $(0.3 \mu \mathrm{m}$ section of Spurr's low viscosity medium) containing known concentration ( $10 \mathrm{mM})$ of metal-organic complexes of $\mathrm{Li}, \mathrm{Na}, \mathrm{Sr}$, and $\mathrm{Pb}$. Inset demonstrates mass resolution at the example of the $\mathrm{Pb}$-isotopes.

Detection limits for a number of elements of special interest are listed in Table 1. רe values demonstrate that one of the features of the LAMMA instrument is the combination $f$ very high relative as well as absolute sensitivity. Several hundred to thousand atoms f interest in the sampled volume will usually suffice for detection. Detection of trace lements in organic matrices may be a problem if their signals are obscured by that of ragment ions of nominally equal mass (for matrices of known composition background ubtraction can at least partially remedy this limitation).

At the present state of development, quantitation of the results poses considerable ifficulties (particularly if absolute quantitation is required). Nevertheless, analys is if standard epoxy specimen containing known concentration of a variety of elements demontrated that a fairly linear signal intensity-concentration plot can be obtained over a ange of about three orders of magnitude (see also Kaufmann et al., 1979 [4]). Relative uantitation should therefore be no problem provided that the instrument parameters are ot changed between measurements and comparable targets are analyzed. For absolute quanitation one must refer to a standard specimen of at least similar general composition, tructure, and geometry.

In contrast to most of the commonly used ionization methods in mass spectrometry, lasernduced ionization yields positive and negative ion signals of about equal intensities. In he LAMMA-instrument spectra of both, ion species can be recorded for successive shots with hanges in polarity of only a few voltages. The intensity of positive ions (e.g., alkaline ons) depends primarily on their ionization potential, that of negative ions (e.g., $\mathrm{Cl}^{-}$) on heir electron affinity. The influence of the target matrix on the signal intensity of tomic ions appears to be small, at least when compared to other methods such as secondary on mass spectrometry (see Burns-Bellhorn, 1978 [5]). Molecules, particularly large organic nes, will generally give typical fragment ions, though parent-molecule and cluster-ion ignals have been recorded for quite a number of non-volatile molecules up to molecular eight of several hundred (see Kupka et al., 1979 [6]). The intensity of signals from large olecules or their fragments depends strongly on the molecular structure and the chemical eactions taking place during the evaporation process. Identification of molecules through 
Table 1. Detection limits of the LAMMA instrument (December 1978).

\begin{tabular}{|c|c|c|c|}
\hline & $\begin{array}{l}\text { Absolute } \\
\text { (g) }\end{array}$ & & $\begin{array}{l}\text { Relative } \\
\text { (ppmw) }\end{array}$ \\
\hline $\mathrm{Li}$ & $2 \times 10^{-} 20$ & & 0.2 \\
\hline $\mathrm{Na}$ & $2 \times 10^{-20}$ & & 0.2 \\
\hline $\mathrm{Mg}$ & $4 \times 10^{-} 20$ & & 0.4 \\
\hline Al & $2 \times 10^{-} 20$ & & 0.2 \\
\hline K & $1 \times 10^{-20}$ & & 0.1 \\
\hline $\mathrm{Ca}$ & $1 \times 10^{-19}$ & & 1.0 \\
\hline $\mathrm{Cu}$ & $2 \times 10^{-18}$ & $\left(10^{-17}\right)$ & $20.0(200)$ \\
\hline $\mathrm{Rb}$ & $5 \times 10^{-20}$ & & 0.5 \\
\hline Cs & $3 \times 10^{-20}$ & & 0.3 \\
\hline $\mathrm{Sr}$ & $5 \times 10^{-20}$ & $\left(10^{-19}\right)$ & $0.5(5.0)$ \\
\hline $\mathrm{Ag}$ & $1 \times 10^{-19}$ & & 1.0 \\
\hline $\mathrm{Ba}$ & $5 \times 10^{-} 19$ & & 0.5 \\
\hline $\mathrm{Pb}$ & $1 \times 10^{-19}$ & & 0.3 \\
\hline u & $2 \times 10^{-19}$ & & 2.0 \\
\hline
\end{tabular}

NOTE: Data refer to an analyzed volume of $\sim 3 \times 10^{-13} \mathrm{~g}$ of (organic matrix) material and to the condition that polyatomic fragments of the matrix do not interfere with the atomic mass signals to be measured. Data in parentheses refer to a standard reference specimen prepared by Spurr's low viscosity medium. Here organic "background" peaks interfere with the Cu- and Sr-signal.

fingerprinting methods is nevertheless possible as the fragmentation patterns and relative signal intensities are quite reproducible for a given molecule and instrument setting. The complementary information contained in the spectra of positive and negative ions is helpful in this respect.

Ion yield, initial energy distribution of the ions and fragmentation of larger molecules depend critically on the laser parameters, particularly on the irradiance in the focus. The irradiances of $10^{8}-10^{11} \mathrm{Wcm}^{-2}$ used in the LAMMA instrument are well in the range where classical target absorption at the laser wavelength is not a necessary prerequisite for the energy transfer leading to evaporation and partial ionization of the target volume. Depending on the target geometry and general type of target material (i.e., transparent dielectric, metal, etc.) an optimal irradiance can always be found with a few test shots. Though the details of the interaction process are not yet fully understood, experience has shown that irradiances close to the threshold of ion formation will preferentially desorb ions from the target surface facing the spectrometer. Irradiances a factor of 3-10 above threshold will lead to an evaporation of the full target volume and yield information on the bulk composition. For small particles, reproducible surface desorption may be quite difficult, and the latter mode of operation is the preferred one in most cases. 
Performance and Limitation of the LAMMA Technique as Applied to Particulate Materials

With respect to the special requirements for particle analysis, laser microprobe mass analysis offers a number of advantages if compared with alternative techniques:

- As far as atomic composition is concerned, all elements of the periodic table can be detected, many of them at sensitivities in the ppmw or even sub-ppmw range (see Table 1). This usually allows a high sensitivity for the detection of many trace contaminants in single particles.

- Since the LAMMA technique separates isotopes, isotopic ratio studies can be performed. For instance, stable isotopes may be used in isotope dilution studies of kirietic processes in aerosol systems, or changes in natural isotopic abundances during the history of particle formation may be detected.

- For the chemical characterization of aerosol particles with the aim to establish their origin, history, and ecological significance, element analysis is usually not sufficient. In this regard cne needs as much information as possible about the molecular constituents (both organic and inorganic). Surely, an ideal case would be the possibility to detect, e.g., the presence of carcinogenic or toxic compounds. Since one of the unique features of LAMMA is that one obtains not only classical organic fingerprint spectra, but, under favorable conditions and appropriate instrument setting, also very characteristic and simple "desorption" spectra of major organic constituents, there is scme hope that this could provide for a considerable step ahead in the organic microprobe analysis of particulate materials.

- If microprobe techniques are applied to single particle analysis, usually the problem of distribution statistics is raised. If the measuring procedure or the time required to perform one analysis exclude the possibility to analyze larger populations of individual particles there is always a loss of important statistical information. Therefore, the fact that with the LAMMA instrument the time needed for the analysis is limited only by data processing or writing the recorded mass spectra (at the time usually 30-60 s), a large number of analyses can be executed in a reasonable period of time.

- In many studies one wants to know particle morphology (size and shape), the distribution of particles over the support or the exact position of individual particles chosen for analysis, either as independent information or in correlation with the analytical data. This requires a suitable imaging technique. In the LAMMA instrument we can obtain such structural information which a light microscope can deliver, including phase contrast, polarization contrast, and dark field illumination and, most importantly, all techniques of optical pattern analysis can be easily fitted to the optical part of the LAMMA instrument (see e.g., McCrone and Delly, 1973 [7]; Morton and McCarthey, 1975 [8]). If the structural elements of the particles or the whole particle itself are below the spatial resolution of the optical microscope, there is still the possibility left to use size dispersive devices for controlled particle deposition (e.g., Cadle, 1975 [9]; Mercer, 1973 [10]) or, preferably, to make a scanning electron microscope (SEM) picture of the particle(s) to be analyzed prior to the LAMMA analysis.

There are also some limitations and disadvantages of the LAMMA technique either based on physical or instrumental conditions.

- LAMMA analysis is essentially destructive. This, for instance, excludes the possibility of recording the spectra of positive and negative ions from the same particle. Furthermore, optimizing the operational conditions of the instrument (e.g., adjusting the optimal power density) usually requires some test shots with the unavoidable loss of a corresponding number of particles.

- The fact that (up to $\sim 2 \mu \mathrm{m}$ particle size) the whole particle is lost at once may sometimes turn into a disadvantage, particularly if one aims at surface analysis. With larger particles, however, it has been found that LAMMA allows for some modest kind of depth profiling (at least under favorable conditions). 
- As in most other microprobe techniques, quantitative analysis imposes many problems. In the absence of a model for the laser-induced plasma formation, an empirical approach is the only practical way. However, approaches to semiquantitative analysis are possible on the basis that (1) matrix effects on ion yield are much less important in LAMMA than they are in SIMS, (2) intensity-concentration plots for elements are linear over several orders of magnitude at least in standard specimen, and (3) reference to standards is possible whenever appropriate reference materials are available.

- With every laser-induced evaporation of an individual particle, part of the supporting film is also evaporated (see figure 3 ). This material, of course, contributes to the recorded LAMMA-spectrum and inevitably creates some kind of a "background." The ratio of these background signals to mass signals originating from the particle itself depends on the size of the particle, the thickness of the supporting foil, and the chemical nature of both materials. Since formvar, collodium, or carbon foils can be made as thin as some $100 \AA$ there is usually no background problem encountered with particles in the range down to about $0.5 \mu \mathrm{m}$ in size. If particles become smaller or if trace constituents are to be analyzed, the foil-induced background can be no longer neglected. However, since the LAMMA spectra of the supporting foil are usually well defined and highly reproducible, methods of background subtraction can be successfully applied.

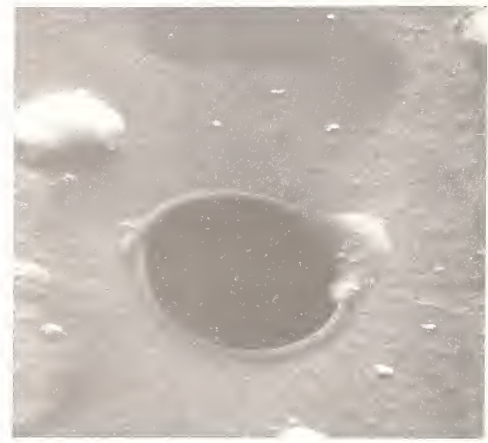

Figure 3. SEM picture of aerosol particles deposited on a thin (500 Ă) formvar foil. Note the hole at the (former) location of a particle. Diameter of the hole is about twice $(2 \mu \mathrm{m})$ the size of the evaporated particle.

- The need to bring the specimen into vacuum will create problems when the analysis of liquid or volatile particles is of interest. However, it should not be too difficult to design a suitable cold stage for LAMMA analysis of particles partially or fully in the liquid phase.

\section{LAMMA Applications in Particle Analysis}

Due to the short period of availability of the LAMMA instrument only a few scattered applications to particle analysis have been performed so far. Considering the data presented in the subsequent paragraphs it should further be noted that the understanding of the mechanisms involved in laser-induced ionization is as yet limited. Assignments of mass signals to specific molecules or atoms may therefore be preliminary only in some cases (besides many unambiguous identifications). 

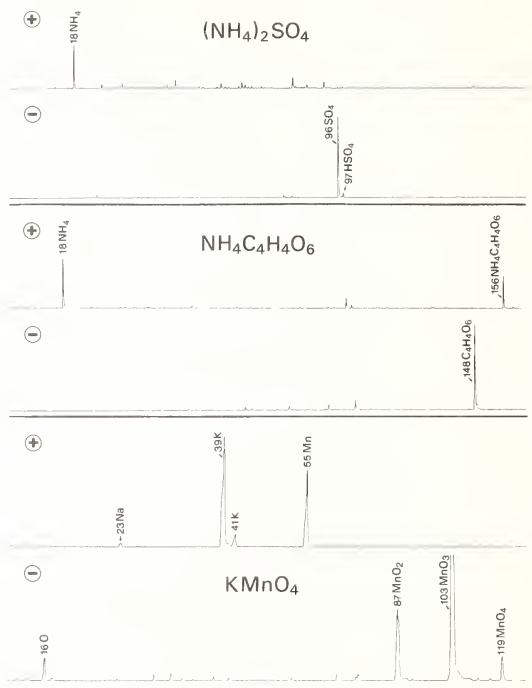

Figure 5. LAMMA spectra of positive and negative ions as recorded from various reference particles (2 $\mu \mathrm{m}$ size) of $\left(\mathrm{NH}_{4}\right)_{2} \mathrm{SO}_{4}, \mathrm{NH}_{4} \mathrm{C}_{4} \mathrm{H}_{4} \mathrm{O}$ and $\mathrm{KMnO}_{4}$. Laser irradiance three times above threshold for detectable ion formation. 

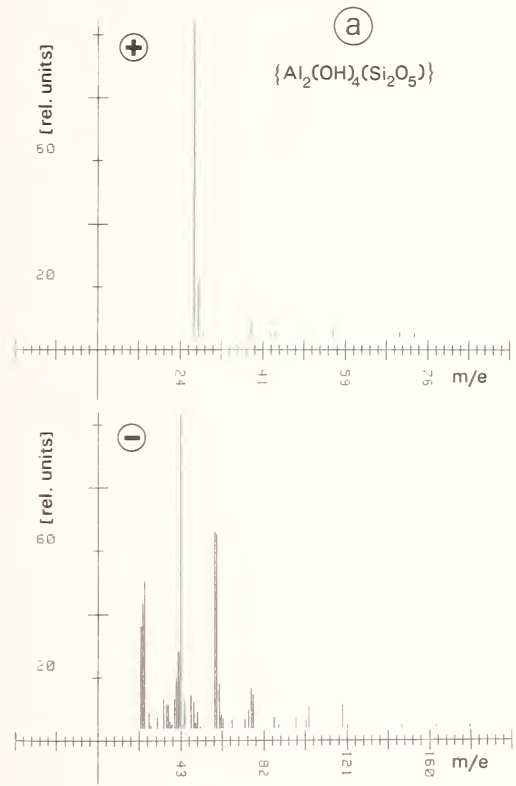

Figure 6a. Average mass spectrum of positive ions (upper panel) and negative ions ('ower panel) obtained from kaolinite particles $\left(\mathrm{Al}_{2}(\mathrm{OH})_{4}\left\{\mathrm{Si}_{2} \mathrm{O}_{3}\right\}\right.$ ) (size $1 \mu \mathrm{m}$ ) deposited on a $500 \AA$ formvar foil. Computer evaluation and peak assignments, see Table 3 . 


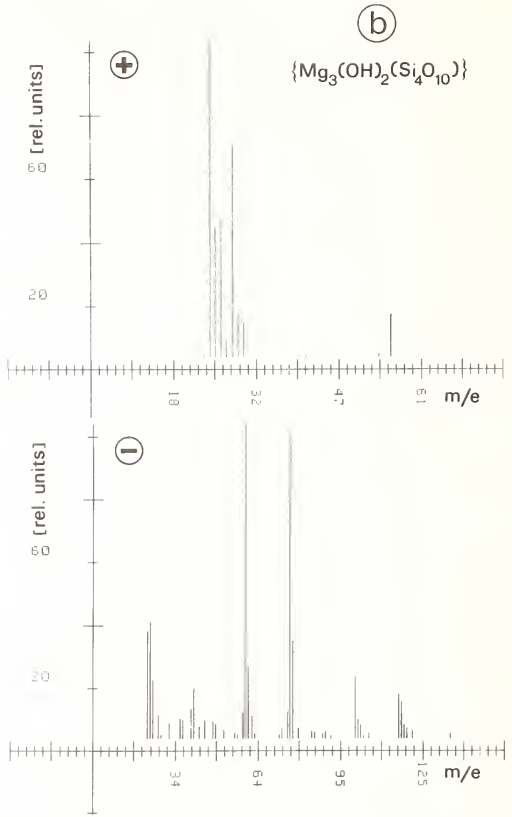

Figure 6b. Average mass spectrum of positive ions (upper panel) and negative ions (lower pane1) obtained from talcum $\left(\mathrm{Mg}_{3}(\mathrm{OH})_{2}\left\{\mathrm{Si}_{4} \mathrm{O}_{10}\right\}\right)$ powder particles (size 4-5 $\mu \mathrm{m}$ ) deposited on a $500 \AA$ formvar foil. Computer evaluation and peak assignments, see Table 4. 
The LAMMA spectra of silicate (reference) particles (see figures 6a,b) are more omplex, although fairly reproducible fingerprint spectra have been obtained in both $\left.\mathrm{Al}_{2}(\mathrm{OH})_{4}\left\{\mathrm{Si}_{2} \mathrm{O}_{5}\right\}\right)$ and $\left(\mathrm{Mg}_{3}(\mathrm{OH})_{2}\left\{\mathrm{Si}_{4} \mathrm{O}_{10}\right\}\right)$ particles. Nevertheless, most of the prominent eaks can be unequivocally assigned in the spectra of the positive as well as of the regative ions. The signal intensity of the $\mathrm{Si}^{+}$ion is rather low (at least at the laser rradiance of $3 \times$ threshold chosen). At higher irradiances (10-15 times threshold) the ield of positive ${ }^{28} \mathrm{Si}^{+}$ions increases in relation to the negative silicon containing iolecular ions.

It is interesting to compare the $\left(\mathrm{NH}_{4}\right)_{2} \mathrm{SO}_{4}$ LAMMA spectra with those obtained by SIMS rom equal reference particles investigated in a recent Cambridge Instrument study (1978) ${ }^{2}$ If upper-atmospheric fly-ash particles. Similar to the LAMMA spectrum, a prominent ${ }^{18} \mathrm{NH}_{4}{ }^{+}$ ignal was recorded in the SIMS spectrum of positive ions (together with strong ${ }^{1} \mathrm{H}^{+}$and ${ }^{7} \mathrm{NH}_{3}{ }^{+}$signals not seen in the LAMMA spectrum). Since negative secondary ions are not asily available in SIMS, the information related to the ${ }^{96} \mathrm{SO}_{4}{ }^{-}$group is contained only in number of very small signals assignable to a large variety of positively charged lolecules consisting of $\mathrm{S}$ in any possible combination with $\mathrm{H}, \mathrm{N}$, and 0 .

The above LAMMA spectra of reference particles have been taken at laser irradiances lear threshold (for evaporating material). Figure 7 compares the spectrum of one type of hese particles $\left\{\left(\mathrm{NH}_{4}\right)_{2} \mathrm{SO}_{4}\right\}$ taken at an irradiance of about 10 times threshold intensity ith that recorded at low irradiance ( 3 times threshold). This spectrum is completely lifferent, more complicated and much less suited for a straightforward chemical identificaion. However, although here the primary information obtained in the low irradiance spectra s lost, one gains the advantage that, in the analysis of particles with unknown chemical :omposition, atomic trace contaminants (for instance heavy metals) would show up under these onditions at optimal detection limits (ppmw to sub-ppmw in favourable cases, such as $\mathrm{Pb}$ or $U$ ).
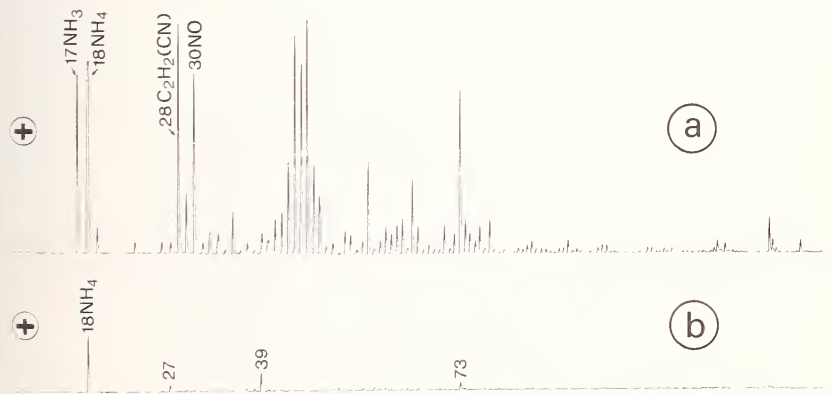

igure 7. Positive mass spectra of $\left(\mathrm{NH}_{4}\right)_{2} \mathrm{SO}_{4}$ reference particles recorded at low laser irradiance ( 3 times threshold, lower panel) and at high irradiance (10 times threshold, upper pane1). See text for further information.

SIMS Studies and Structural Identification of Compounds in Upper Atmosphere Fly Ash, 1.17 ASRS, Cambridge Instrument. 
Subsequent1y, Weiser et a1., 1979 [11], analyzed a randomly chosen population of 100 single aerosol particles between $0.5 \mu \mathrm{m}$ and $1 \mu \mathrm{m}$ (1arge particles) and $1 \mu \mathrm{m}-10 \mu \mathrm{m}$ (giant particles) in size.

In figures 8 and 9 a collection of LAMMA spectra are shown demonstrating typical records of either positive or negative ions in both large and giant particles. Tables 2a and $2 \mathrm{~b}$ list the relative frequency at which signals of the most frequent mass numbers appear in the spectra of the investigated aggregate of 100 analyzed particles. In this evaluation all signals of an intensity of about 10 percent full scale were counted. Possible molecular assignments were given for most of the mass numbers but, as mentioned before, are still the object of some uncertainty.

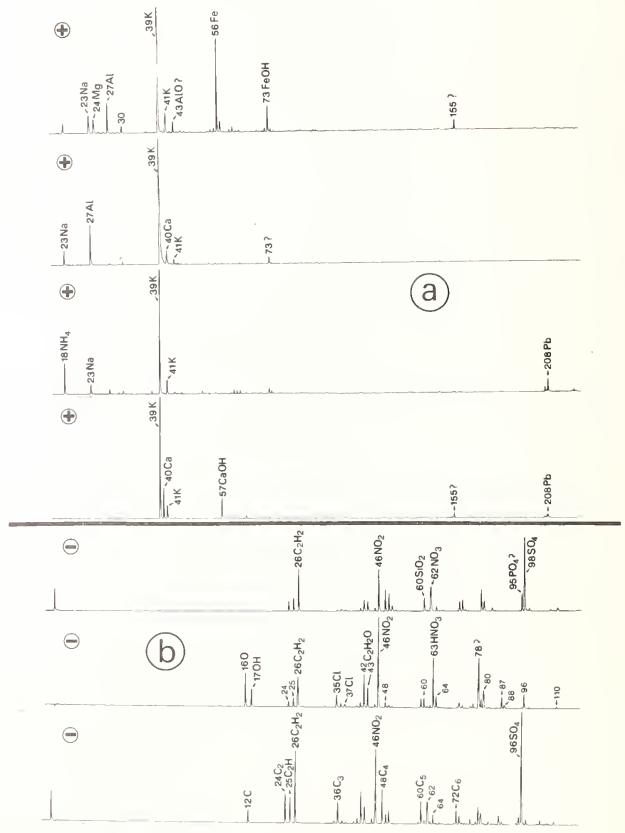

Figure 8. LAMMA spectra of (large) aerosol particles (size $0.5-1 \mu \mathrm{m}$ ). Positive ions spectra in the upper part; negative ions spectra in the lower part. See text for further information. 


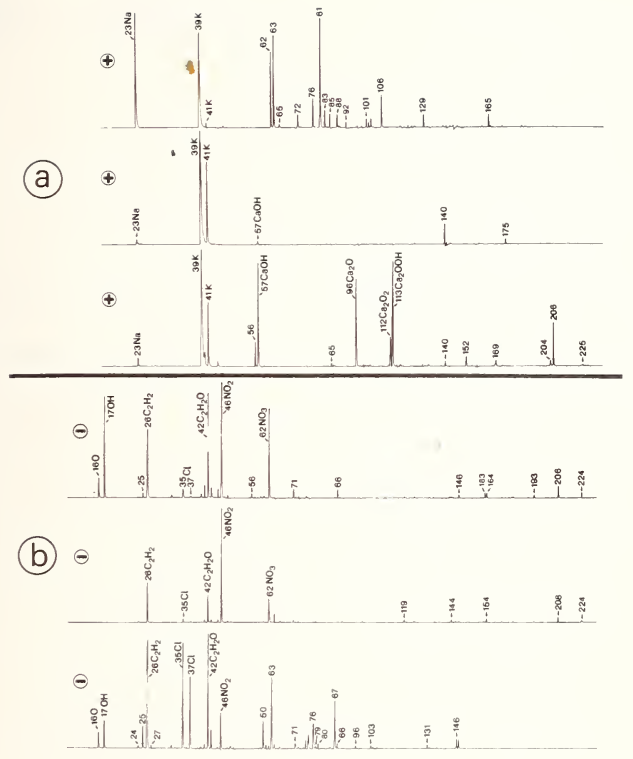

=igure 9. LAMMA spectra of (giant) aerosol particles (size 1-10 $\mu \mathrm{m}$ ). Positive ions spectra in the upper part, negative ions spectra in the lower part. See text for further information. 
Table 2a. Relative frequency of appearance (above $10 \%$ full scale) of mass signals in the spectra of positive ions recorded in 100 aerosol particles.

\begin{tabular}{|c|c|c|c|c|c|}
\hline $\mathrm{m} / \mathrm{e}$ & $\begin{array}{l}\text { Frequency } \\
\text { (percent) }\end{array}$ & $\begin{array}{l}\text { Ions which may } \\
\text { represent the } \\
\text { measured m/e-values }\end{array}$ & $\mathrm{m} / \mathrm{e}$ & $\begin{array}{l}\text { Frequency } \\
\text { (percent) }\end{array}$ & $\begin{array}{l}\text { Ions which may } \\
\text { represent the } \\
\text { measured } \mathrm{m} / \mathrm{e} \text {-values }\end{array}$ \\
\hline 18 & 71 & $\mathrm{NH}_{4}^{+}, \mathrm{H}_{2} \mathrm{O}^{+}$ & 60 & A. 10 & \\
\hline 23 & 84 & $\mathrm{Na}^{+}$ & 61 & 10 & \\
\hline 24 & 19 & $\mathrm{Mg}^{+}$ & 62 & 13 & \\
\hline 25 & 19 & $\mathrm{Mg}^{+}$ & 63 & 13 & $\mathrm{Cu}^{+}$ \\
\hline 26 & 19 & $\mathrm{Mg}^{+}$ & 65 & 13 & $\mathrm{Cu}^{+}$ \\
\hline 27 & 52 & $\mathrm{Al}^{+}$ & 69 & 10 & \\
\hline 28 & 29 & $\mathrm{Si}^{+}, \mathrm{C}_{2} \mathrm{H}_{4}^{+}, \mathrm{CO}^{+}$ & 72 & 13 & $\mathrm{FeO}^{+}$ \\
\hline 29 & 29 & $\mathrm{C}_{2} \mathrm{H}_{5}^{+}$, & 73 & 29 & $\mathrm{FeOH}^{+}$ \\
\hline $\begin{array}{l}30 \\
31\end{array}$ & $\begin{array}{l}39 \\
26\end{array}$ & $\begin{array}{l}\mathrm{C}_{4} \mathrm{H}_{6}{ }^{+}, \mathrm{CH}_{2} \mathrm{OH}^{+} \\
\mathrm{CN}^{+}, \mathrm{CH}_{2} \mathrm{O}^{+}\end{array}$ & $\begin{array}{l}74 \\
85\end{array}$ & $\begin{array}{l}10 \\
13\end{array}$ & \\
\hline 39 & 100 & $\mathrm{~K}^{+}$ & 91 & 10 & \\
\hline 40 & 26 & $\mathrm{Ca}^{+}$ & 97 & 10 & \\
\hline 41 & 100 & $k^{+}$ & 112 & 10 & \\
\hline 43 & 45 & $\mathrm{~A} 10^{+}$ & 128 & 10 & \\
\hline 44 & 19 & $\mathrm{AlOH}^{+}, \mathrm{CO}_{2}, \mathrm{Ca}$ & 140 & 10 & \\
\hline 45 & 13 & $\mathrm{SiOH}^{+}$ & 152 & 10 & \\
\hline 51 & 10 & & 155 & 10 & \\
\hline 55 & 35 & $\mathrm{Mn}^{+}$ & 206 & 29 & $\mathrm{~Pb}^{+}$ \\
\hline 56 & 42 & $\mathrm{Fe}^{+}, \mathrm{CaO}^{+}$ & 207 & 29 & $\mathrm{~Pb}^{+}$ \\
\hline 57 & 35 & $\mathrm{CaOH}^{+}, \mathrm{MgO}_{2}{ }^{+}$ & 208 & 29 & $\mathrm{~Pb}^{+}$ \\
\hline
\end{tabular}


Table $2 b$. Relative frequency of appearance ( $10 \%$ full scale) of mass signals in the spectra of negative ions recorded in 100 aerosol particles.

\begin{tabular}{|c|c|c|c|c|c|}
\hline $\mathrm{m} / \mathrm{e}$ & $\begin{array}{l}\text { Frequency } \\
\text { (percent) }\end{array}$ & $\begin{array}{l}\text { Ions which may } \\
\text { represent the } \\
\text { measured } \mathrm{m} / \mathrm{e} \text {-values }\end{array}$ & $\mathrm{m} / \mathrm{e}$ & $\begin{array}{l}\text { Frequency } \\
\text { (percent) }\end{array}$ & $\begin{array}{l}\text { Ions which may } \\
\text { represent the } \\
\text { measured } \mathrm{m} / \mathrm{e} \text {-values }\end{array}$ \\
\hline 1 & 16 & $\mathrm{H}^{-}$ & 63 & 32 & $\mathrm{HNO}_{3}^{+}, \mathrm{PO}_{2}^{+}, \mathrm{C}_{3} \mathrm{H}_{3}^{+}$ \\
\hline 12 & 3 & $c^{-}$ & 64 & 58 & $\mathrm{SO}_{2}^{-}$ \\
\hline 13 & 3 & $\mathrm{CH}^{-}$ & 70 & 10 & \\
\hline 16 & 32 & $0^{-}, \mathrm{NH}_{2}^{-}$ & 71 & 16 & \\
\hline 17 & 52 & $\mathrm{OH}^{-}$ & 72 & 61 & $\mathrm{C}_{6}^{-}$ \\
\hline 19 & 3 & $\mathrm{~F}^{-}$ & 73 & 45 & $\mathrm{C}_{6} \mathrm{H}^{-}$ \\
\hline 24 & 68 & $\mathrm{C}_{2}^{-}$ & 74 & 19 & $\mathrm{C}_{6} \mathrm{H}_{2}^{-}$ \\
\hline 25 & 68 & $\mathrm{C}_{2} \mathrm{H}^{-}$ & 75 & 13 & $\mathrm{C}_{6} \mathrm{H}_{3}^{-}$ \\
\hline 26 & 81 & $\mathrm{C}_{2} \mathrm{H}_{2}^{-}, \mathrm{CN}^{-}$ & 76 & 26 & $\mathrm{SiO}_{3}^{-}$ \\
\hline 27 & 32 & $\mathrm{C}_{2} \mathrm{H}_{3}^{-}$ & 77 & 10 & $\mathrm{HSiO}_{3}^{-}$ \\
\hline 32 & 19 & $s^{-}$ & 78 & 10 & \\
\hline 35 & 61 & $\mathrm{Cl}^{-}$ & 79 & 16 & $\mathrm{PO}_{3}{ }^{-}$ \\
\hline 36 & 48 & $\mathrm{Cl}_{3}^{-}$ & 80 & 81 & $\mathrm{SO}_{3}^{-}$ \\
\hline 37 & 61 & $\mathrm{CL}^{-}, \mathrm{C}_{3} \mathrm{H}^{-}$ & 81 & 61 & $\mathrm{HSO}_{3}^{-}$ \\
\hline 40 & 65 & $\mathrm{CN}_{2}^{-}$ & 83 & 23 & \\
\hline 41 & 71 & $\mathrm{C}_{2} \mathrm{HO}^{-}$ & 87 & 10 & \\
\hline 42 & 71 & $\mathrm{C}_{2} \mathrm{H}_{2} \mathrm{O}^{-}$ & 88 & 16 & \\
\hline 43 & 71 & $\mathrm{C}_{2} \mathrm{H}_{3} \mathrm{O}^{-}$ & 96 & 87 & $\mathrm{SO}_{4}^{-}$ \\
\hline 45 & 71 & & 107 & 13 & \\
\hline 46 & 87 & $\mathrm{NO}_{2}^{-}$ & 110 & 26 & \\
\hline 48 & 71 & $\mathrm{C}_{4}^{-}, \mathrm{SO}^{-}$ & ווו & 26 & \\
\hline 49 & 55 & & 119 & 13 & \\
\hline 50 & 55 & & 120 & 19 & \\
\hline 51 & 10 & & 146 & 10 & \\
\hline 59 & 35 & $\mathrm{CH}_{3} \mathrm{COO}^{-}$ & 147 & 10 & \\
\hline 60 & 65 & $\mathrm{C}_{5}^{-}, \mathrm{CO}_{3}^{-}, \mathrm{SiO}_{2}^{-}$ & 164 & 10 & \\
\hline 61 & 48 & $\mathrm{C}_{5} \mathrm{H}^{-}, \mathrm{HCO}_{3}^{-}$ & 208 & 16 & \\
\hline 62 & 81 & $\mathrm{C}_{5} \mathrm{H}_{2}{ }^{-}, \mathrm{NO}_{3}{ }^{-}$ & 224 & 16 & $\mathrm{PbO}^{-}$ \\
\hline
\end{tabular}


Table 3. Computer evaluation of average spectrum shown in figure 6a.

Assignment Area of Peak Standard Deviation (percent)
Standard Deviation (percent)

Positive Ions

$\begin{array}{ll}\text { Mass } & 27 \\ \text { Mass } & 28 \\ \text { Mass } & 29 \\ \text { Mass } & 39 \\ \text { Mass } & 41 \\ \text { Mass } & 43 \\ \text { Mass } & 44 \\ \text { Mass } & 56 \\ \text { Mass } & 70 \\ \text { Mass } & 73\end{array}$

A1

Si

Si

A10

Negative Ions

Mass 24

Mass 25

Mass 26

Mass 28

Mass 32

Mass 35

Mass 36

Mass 37

Mass 40

Mass 41

Mass 42

Mass 43

Mass 45

Mass 48

Mass 49

Mass 51

Mass 59

Mass 60

Mass 61

Mass 62

Mass 63

Mass 67

Mass 73

Mass 75

Mass 76

Mass 77

Mass 87

Mass 97

Mass 102

Mass 103

Mass 119

$\bar{a}(f)=$ foil.
$C_{2}(f)^{a}$

$\mathrm{C}_{2} \mathrm{H}$ (f)

$\mathrm{C}_{2} \mathrm{H}_{2}$ (f)

$\mathrm{S}$

C1

$\mathrm{C}_{3}$ (f)

$\mathrm{Cl}, \mathrm{C}_{3} \mathrm{H}(\mathrm{f})$

$\mathrm{C}_{2} \mathrm{HO}$ (f)

A10

(f)

(f)

$\mathrm{AlO}_{2}$

$\mathrm{SiO}_{2}$

$\mathrm{A}^{10} \mathrm{O}_{3}$

$\mathrm{SiO}_{3}$

$\mathrm{HSiO}_{3}$

$\mathrm{SiAlO}_{3}$

$\mathrm{SiAlO}_{4}$
3882.0

710.0

53.3

238.7

25.3

61.3

55.3

88.0

64.7

54.7

572.0

701.6

822.0

91.2

61.6

165.2

138.8

137.2

163.2

284.8

428.0

1773.2

152.4

186.8

148.6

92.6

1099.2

1086.0

245.4

75.6

55.6

48.4

48.8

101.8

224.8

188.4

61.6

60.4

47.6

125.6

137.6
391.96

124.24

17.95

29.54

7.40

18. 30

32.49

22.03

9. 40

21.78

128. 98

158.54

292.01

21.52

21.17

65.86

55.07

43.00

53.02

58.71

206.06

238.53

22.93

47.26

40.25

11.44

167.86

189.95

53.44

8.90

9.50

9.07

6.81

17.56

27.85

44.79

15.07

31.19

12. 36

33. 10

33.44
10.6

17.9

33.7

12. 3

29.2

29.8

58.7

25.0

14.5

39.8

22.5

22.6

35.5

23.6

34. 4

39.9

39.7

31.3

32.5

20.6

48.1

13.5

15.0

25.3

27.1

12.4

15.3

17.5

21.8

11.8

17.1

18.7

14.0

17.3

12.4

23.8

24.5

51.6

26.0

26.4

24.3 
Table 4. Computer evaluation of average spectrum shown in figure $6 b$.

Assignment Area of Peak Standard Deviation
Standard Deviation

(percent)

Positive Ions

$\begin{array}{lllr}\text { Mass } & 23 & \mathrm{Na} & 40.5 \\ \text { Mass } & 24 & \mathrm{Mg} & 4074.7 \\ \text { Mass } & 25 & \mathrm{Mg} & 1670.0 \\ \text { Mass } & 26 & \mathrm{Mg} & 1759.0 \\ \text { Mass } 27 & \mathrm{Al} & 212.0 \\ \text { Mass } & 28 & \mathrm{Si} & 2697.5 \\ \text { Mass } & 29 & \mathrm{Si} & 553.5 \\ \text { Mass } & 30 & \mathrm{Si} & 440.0 \\ \text { Mass } 54 & & 45.0 \\ \text { Mass } 56 & \mathrm{MgO}_{2} & 551.0\end{array}$

19.87

194.82

168.81

278.84

93.60

704.15

128.00

116.88

13.67

149.55

49. 1

4.8

10.1

15.9

44.2

26. 1

23. 1

26.6

30.4

27. 1

Negative Ions

Mass 24

Mass 25

Mass 26

Mass 28

Mass 32

Mass 36

Mass 37

Mass 40

Mass 41

Mass 43

Mass 45

Mass 48

Mass 49

Mass 52

Mass 59

Mass 60

Mass 61

Mass 62

Mass 73

Mass 75

Mass 76

Mass 77

Mass 79

Mass 84

Mass 100

Mass 101

Mass 102

Mass 116

Mass 117

Mass 118

Mass 119

Mass 121

$\mathrm{C}_{2}(\mathrm{f})^{\mathrm{a}}$

$\mathrm{C}_{2} \mathrm{H}$ (f)

$\mathrm{C}_{2} \mathrm{H}_{2}$ (f)

628.0

680.1

342.4

139.2

88.0

$\mathrm{C}_{3}$ (f)

$\mathrm{C}_{3} \mathrm{H}(\mathrm{f})$

118.0

106.8

174.8

$\mathrm{C}_{2} \mathrm{HO}$ (f)

294.8

71.2

106.8

107.2

(f)

85.2

48.0

151. 2

$\mathrm{SiO}_{2}$

1838.4

417.2

129.6

60.0

152.4

201.67

240.29

162.91

62.94

50.17

55.97

32.94

84.35

86.37

24.18

29.58

50.85

50.36

23.63

33.53

32.1

35. 3

47.6

45. 2

57.0

47.4

30.8

48.3

29.3

34.0

27.7

47.4

59.1

49.2

22.2

454.85

79.56

49.17

16.26

41.77

24.7

19.1

37.9

27.1

27.4

1797.6

$\mathrm{SiO}_{3}$

573.2

60.0

44.0

$\mathrm{SiMgO}_{3}$

359.2

112.8

80.0

$\mathrm{SiMgO}_{4}$

262.8

214.8

77.6

452. 36

110.04

15.85

12.71

83.77

25.4

19.2

26.4

28.9

23.3

28.14

24.9

21.80

27.2

90.39

34.4

64.20

29.9

27.17

35.0

58.4

30.72

19.73

52.6

48.4

40.8

${ }^{a}(f)=$ foil. 
The LAMMA spectra of large and giant particles are basically similar. However, in large particles there is a higher incidence of metallic constituents such as $\mathrm{Na}, \mathrm{Mg}, \mathrm{Al}$, $\mathrm{Ca}, \mathrm{Ni}, \mathrm{Cu}, \mathrm{Zn}$, and $\mathrm{Cd}$, and a deficiency of $\mathrm{NO}$ and $\mathrm{NO}_{3}$, whereas in giant particles the incidence of $\mathrm{SO}_{4}, \mathrm{HSO}_{4}, \mathrm{NH}_{4}$, and $\mathrm{Pb}$ was smaller if compared with large particles.

Among a particle population of common size and appearance a few particles can always be found, the compositions of which considerably differ from that of the majority of the population. Examples for such kind of "strange" particles are given in figure 10. The LAMMA spectrum shown in part (a) of figure 10 reveals a particle of obviously industrial origin with the large signals of $\mathrm{Zn}$ and $\mathrm{Fe}$ not observed in other spectra of the investigated population. In view of the high ionization potential of $\mathrm{Zn}$, the appearance of an intensive Zn signal suggests a much higher $\mathrm{Zn}$ content than one would suspect from the signal intensity ratios to $\mathrm{Na}, \mathrm{Al}, \mathrm{K}$, and $\mathrm{Fe}$ which are also present in this particular spectrum. $\mathrm{A}$ completely different composition and origin must be attributed to the particle from which the LAMMA spectrum of figure $10 \mathrm{~b}$ was recorded. The few (saturated) signals of positive ions ( $\mathrm{Na}, \mathrm{A} 1, \mathrm{~K}, \mathrm{Ca}$, and trace amounts of $\mathrm{Mg}$ ) suggest it's originating from the soil. Finally, in the spectrum of negative ions shown in figure 10c, a typical "fly-ash" particle is fingerprinted with its characteristic pattern of carbon molecules up to $V_{10}$. It is further interesting to note that in this particular spectrum an unequivocal ${ }^{19} \mathrm{~F}^{-}$signal appears which (if related to results obtained in standard reference specimen of known fluorine content) would indicate the presence of about 10-50 ppmw of fluorine in the analyzed particle.

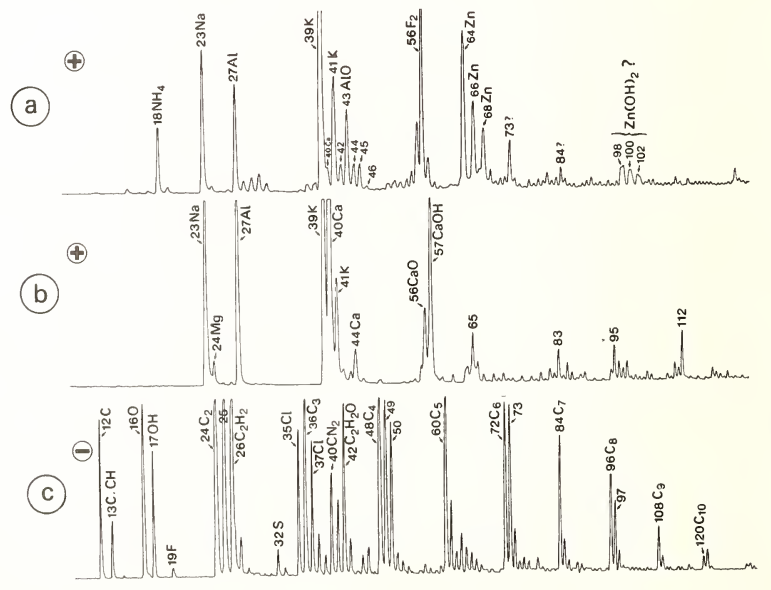

Figure 10. Three LAMMA spectra showing unusual chemical particle composition in a population of 100 analyzed aerosol particles. See text for further explanation. 
In about 30 percent of the analyzed particles lead was detected (see also Table $2 a$ ). There was no obvious association of lead with other constituents. This finding indicates the occurrence of secondary agglomeration between the original (smal1) lead particles formed by nucleation of car exhaust products and other atmospheric particles.

In several occasions the LAMMA spectra of giant particles obtained with the first, second, or third laser shot (applied to the same area of the particle and removing only part of the material present) differ considerably (figure 11). This seems to evidence chemical inhomogeneities within the investigated particle. It also indicates that, under favourable conditions, some kind of a depth profiling may be possible as it has already been shown in LAMMA studies of thin film sandwiches (Kaufmann et al., (1979) [4]).

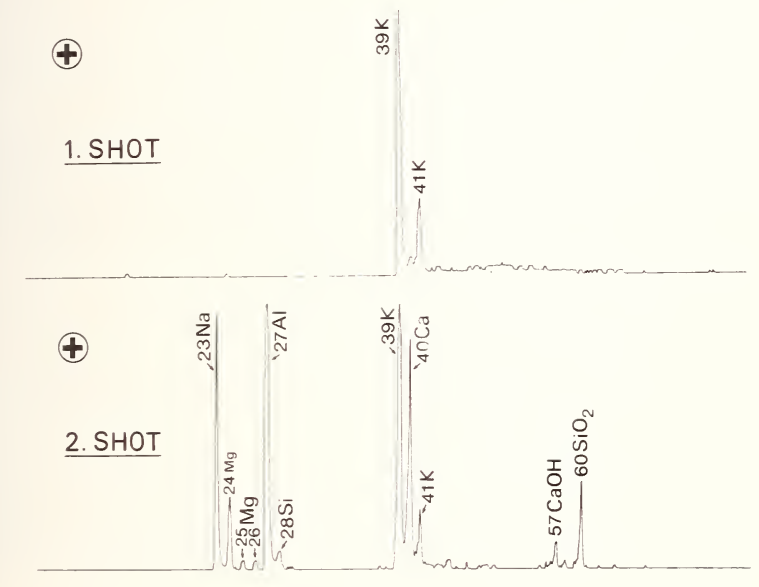

Figure 11. LAMMA spectra of positive ions obtained from a giant aerosol particle (size $8 \mu \mathrm{m})$. Laser irradiance at about three times threshold for ion formation. First shot removes only superficial layer of material, second shot vaporizes material of a deeper layer; particle lost after the third shot. Note the difference in mass spectra recorded with the first (upper panel) and second (lower pane1) laser shot.

\section{Coal Mine Dust Particles in Human Lymphocytes}

Coal worker pneumoconiosis (CWP) has recently attracted new attention from pathologists due to the increasing demand for coal as an alternative energy source in the future (for review see Abraham, 1978 [13]). Coal mine dust particles are certainly a mixed population of mineral and organic particles. There is still a great deal of uncertainty as to the correlation of the pathologic findings to the chemical nature of the particles inducing the various morphological, functional, and, possibly, immunological reactions. In an analytical study of DeNee, Abraham, and Geldermann (1975) [14], the typical nodular lesions observed in lung tissues of patients with CWP were attributed to silica or silicates. 
In a recent LAMMA study, a specimen of cultivated human lymphocytes (courtesy of Prof. Bruch, Institute for Hygiene and 0ccupational Medicine, Gesamthochschule Essen) was investigated, which for eight hours had been incubated in vitro with coal mine dust particles $(0.5-1 \mu \mathrm{m})$. Many of these particles had been taken up by phagocytosis and could be clearly identified intracellularly at different sites in the cytoplasm. The aim of the preliminary study was to find out whether LAMMA could distinguish and, eventually, classify the particles according to their chemical composition and, if so, whether or not lymphocytes would show preferential phagocytosis for particles of a specific composition.

The control analysis of 100 extracellular particles first revealed that roughly about 70 percent of the particles were mineral in nature (figure 12), whereas about 30 percent consisted of pit coal (typical fragmentation pattern of polycarbonhydrates shown in figure 13); only a minor fraction of about 1.2 percent gave "mixed" mass spectra. The population of the mineral particles could be further subdivided by a (somewhat arbitrary) subclassification in which particles were distinguished by the leading elements detected, which were either $\mathrm{Al},(\mathrm{Si}), \mathrm{K}$, and $\mathrm{Mn}$ (class (a) in figure 12), or $\mathrm{Na}, \mathrm{Al},(\mathrm{Si}), \mathrm{K}, \mathrm{Mn}$, and $\mathrm{Fe}$ (class (b) in figure 13) or, finally, $\mathrm{Na},(\mathrm{Si}), \mathrm{K}$, and Mn with no Fe present (class (c) in figure 13). With respect to silicon, which is supposed to be one of the major constituents of mineral coal mine dust particles, one must emphasize that signals related to silicon may either show up as ${ }^{28} \mathrm{Si}^{+},{ }^{44}\left(\mathrm{SiO}^{+}\right),{ }^{60}\left(\mathrm{SiO}_{2}\right)^{-},{ }^{76}\left(\mathrm{SiO}_{3}\right)^{-}$, or in many other combinations with Al or $\mathrm{Mg}$ (see also figures $6 \mathrm{a}$ and $6 \mathrm{~b}$ ). Since under the usual operating conditions of the LAMMA instrument (at laser irradiance about 3-5 times above threshold), the ionization potential for $\mathrm{Si}$ is too high for a reasonable ion yield detection, detection limits in the positive ion spectrum are expected to be poor. The situation is further complicated by the fact that in the spectrum of positive ions ${ }^{44}(\mathrm{SiO})$ may be obscured by ${ }^{44} \mathrm{Ca},{ }^{44}\left(\mathrm{Co}_{2}\right)$, and ${ }^{44}(\mathrm{~A} 10 \mathrm{H})$ respectively.
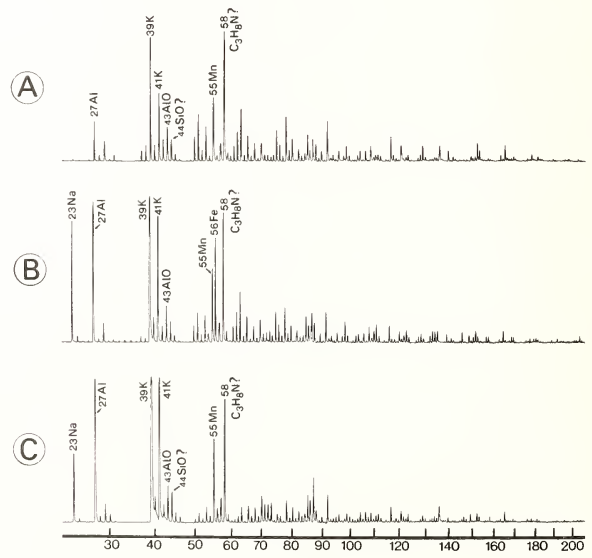

Figure 12. LAMMA spectra of positive ions obtained from (mineral) coal mine dust particles (size 1-2 $\mu \mathrm{m}$ ), which had been administered to cultivated human lymphocytes. 

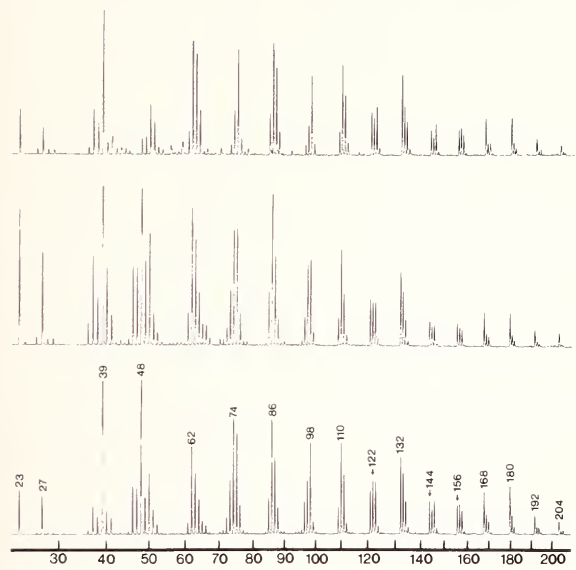

=igure 13. LAMMA spectra of positive ions obtained from organic (pit coal) coal mine dust particles (size 1-2 $\mu \mathrm{m}$ ) which had been administered to cultivated human lymphocytes. Note reproducibility of fragmentation pattern.

As to the second question of preferential phagocytosis, it was demonstrated that pitcoal particles were found inside the lymphocytes twice as often as in the extracellular particle population. This might be an interesting result with respect to the suggestion that immunity mechanisms may be induced by some organic compounds of coal mine dust particles (see Caplan, 1953 [14]; Parkes, 1974 [15]).

\section{Impurity Analysis in Particulate Residues of Solvents}

Monodispersive particles of well defined particle size and homogeneous particle composition can be produced for the calibration of instruments such as optical counters, impactors, or for other aerosol related studies. For this purpose particle generators as described by Berglund and Liu (1973) [17] are used. In such a device, a solution of a non-volatile solute of known concentration in a volatile solvent is sprayed through a vibrating orifice, generating droplets of controllable size with only a small spread of volume. The particles obtained after evaporation of the solvent represent the residue of the non-volatile solute and, of course, are particles of uniform size, shape, and composition. However, if the solvent used already contains unknown amounts of dissolved impurities or undissolved particles, their influence on the properties of the generated particles becomes the more important the smaller their size. An attempt was undertaken to identify the nature of such impurities in a sample of analytical grade (p.a.) isopropanol. In addition, the possibility was to be checked whether LAMMA might be used for a direct trace contaminant analysis in liquid samples, including drinking water.

Figure 14 shows an average spectrum $(n=9)$ of a LAMMA analysis performed in particulate residues $(\sim 1 \mu \mathrm{m}$ size) of analytical grade isopropanol. Leading trace contaminants in this case were $\mathrm{Na}, \mathrm{K}$, and $\mathrm{Ca}$. By reference to the droplet-to-residue volume ratio $\left(\cong 10^{5}\right)$ the total mass fraction of the residue can be estimated roughly to about $15 \mathrm{ppmw}$. Taking 


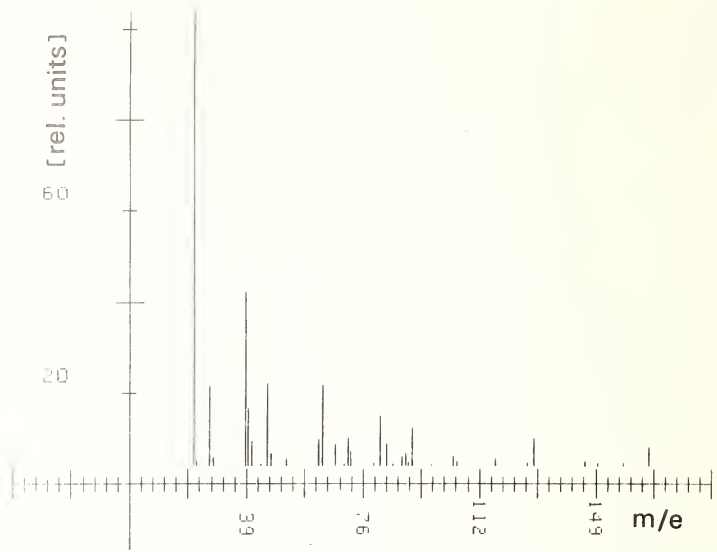

Figure 14. Average mass spectrum (positive ions) of residue particles from sprayed isopropanol (analytical grade); particles (size $1 \mu \mathrm{m}$ ) deposited on $500 \AA$ formvar foil; $n=9$. For computer evaluation and peak assignments see Table 5 .

into account the specific LAMMA sensitivity factors (as they are determined at least for most of the alkaline and earth alkaline metals), a quantitative approximation of the impurity content of the investigated solvent is possible. A few of the particles in the above residue specimen of isopropanol had a completely different composition. They obviously had been already present as dust particles in the liquid sample and consisted mainly of either a Cu-Sn alloy or showed larger signals of $\mathrm{Na}, \mathrm{Ca}, \mathrm{Ba}$, and $\mathrm{Pb}$. In similar studies of particulate residues of drinking water (see figure 15), trace amounts of $\mathrm{Pb}$ could be detected down to a few ppbw with respect to its concentration in the investigated water sample. There was also a remarkable amount of $\mathrm{Sr}$ present in this particular specimen.

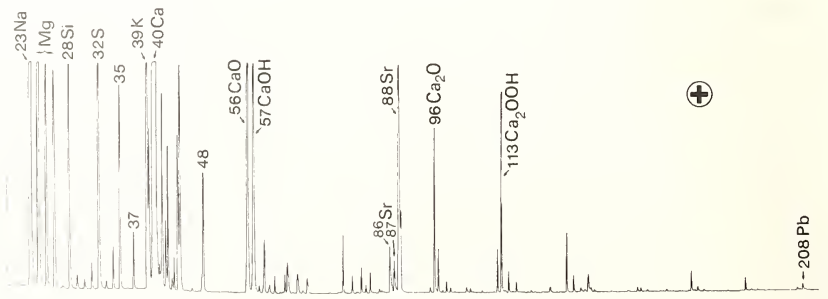

Figure 15. LAMMA spectrum of positive ions as recorded from a residue particle (1 $\mu \mathrm{m}$ size) of a drinking water sample. 
Table 5. Computer evaluation of average spectrum shown in figure 14.

\begin{tabular}{|c|c|c|c|c|c|}
\hline \multicolumn{3}{|r|}{ ent } & Area of Peak & $\begin{array}{l}\text { Standard Deviation } \\
\text { (percent) }\end{array}$ & $\begin{array}{l}\text { Standard Dev } \\
\text { (percent }\end{array}$ \\
\hline \multicolumn{6}{|c|}{ rositive Ions } \\
\hline lass & 23 & $\mathrm{Na}$ & 2763.6 & 80.65 & 2.9 \\
\hline lass & 28 & Si & 480.4 & 163.47 & 34.0 \\
\hline lass & 29 & & 49.1 & 28.89 & 58.8 \\
\hline lass & 39 & K & 1052.6 & 50.74 & 4.8 \\
\hline lass & 40 & $\mathrm{Ca}$ & 346.6 & 16.97 & 4.9 \\
\hline lass & 41 & $\mathrm{~K}$ & 154.0 & 18.28 & 11.9 \\
\hline lass & 46 & & 500.0 & 47.30 & 9.5 \\
\hline Yass & 47 & & 68.1 & 8.22 & 12.1 \\
\hline Yass & 62 & & 160.4 & 28.48 & 17.8 \\
\hline Mass & 63 & & 492.0 & 56.70 & 11.5 \\
\hline lass & 67 & & 123.9 & 19.59 & 15.8 \\
\hline fass & 71 & & 163.0 & 16.57 & 10.2 \\
\hline Mass & 72 & & 84.0 & 11.96 & 14.2 \\
\hline Mass & 81 & & 298.1 & 55.82 & 18.7 \\
\hline Mass & 83 & & 134.5 & 29.27 & 21.8 \\
\hline Mass & 88 & & 56.8 & 9.36 & 16.5 \\
\hline Mass & 89 & & 70.8 & 13.13 & 18.6 \\
\hline Mass & 91 & & 230.9 & 28.16 & 12.2 \\
\hline Mass & 104 & & 57.1 & 8.91 & 15.6 \\
\hline Mass & 105 & & 28.1 & 6.21 & 22.1 \\
\hline Mass & 117 & & 43.1 & 6.33 & 14.7 \\
\hline Mass & 129 & & 169.3 & 20.84 & 12.3 \\
\hline Mass & 165 & & 107.3 & 17.52 & 16.3 \\
\hline
\end{tabular}

Crystalline Organic Particles

An unusual and unique feature of laser-induced microprobe mass analysis is the fact that by control of the laser irradiance in the focus one obtains a variable at hand by which the ionization process and the information contained in the mass spectra of crystalline organic compounds can be easily adjusted to various desirable fragmentation levels. As already mentioned in the analysis of aerosol particles, one can go from "smooth desorption" resulting in very simple and straightforward mass spectra to "full pyrolysis" inducing complicated mass spectra of the highly fragmented organic compounds. Again the high yield of negative ions provides complementary information under all instrumental conditions. Furthermore, difficulties encountered in conventional mass spectrometers when ionizing organic solid state materials of low vapor pressure are circumvented to a certain extent by laser-induced ionization. Finally, a few small particles of 1-10 pg each are sufficient for a full mass spectrometric analysis. Figure 16 demonstrates the example of a fluorinated heterocyclic compound (4-(2'-trifluoromethyl)-2,6-dimethyl-1,4-dihydropyridine-3,5-dicarboxylate) consisting of a pyridine-ring (ring II) and an aromatic (benzene) ring ( $r i n g$ I) bearing a $\mathrm{CF}_{3}$ group. "Smooth laser irradiation" (0.2-1\% transmission) results in mass spectra of a few lines related merely to the two ring systems, whereas with increasing 

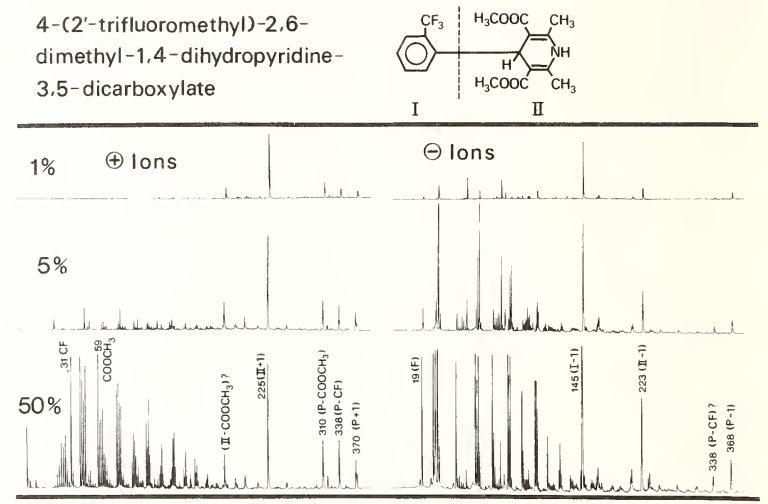

Figure 16. LAMMA spectra of positive and negative ions obtained from microcrystals (1-2 $\mu \mathrm{m})$ of a heterocyclic fluorinated compound. The 1, 5, and 50 percent labels indicate percent laser transmission and, hence, are a measure of the laser irradiance in the focus. See text for further explanation.

laser irradiance fragmentation comes up leading to mass spectra similar to those obtained by electron impact or other conventional ion sources. Again the appearance of some of the larger fragments is rather unexpected according to the rules of classical ionization methods. For instance, the signal at mass number 225 in the spectra of positive ions (related to the pyridine ring) is not easy to explain except for the occurrence of a radical.

The support of this work by the Federal Ministry of Science and Technology is gratefully acknowledged. 


\section{References}

[1] Hillenkamp, F., Unsöld, R., Kaufmann, R., and Nitsche, R., A High Sensitivity Laser Microprobe Mass Analyzer, Appl. Phys. 8, 341-348 (1975).

[2] Heinen, H. J., Wechsung, R., Vogt, H., Hillenkamp, F., and Kaufmann, R., Laser-Mikrosonden-Massen-Analysator LAMMA, Biotechnische Umschau 2, 346-354 (1978).

[3] Wechsung, R., Hillenkarip, F., Kaufmann, R., Nitsche, R., Unsöld, E., Vogt, H., LAMMA - A New Laser-Microprobe-Mass-Analyzer, Microscopica Acta, Supp1. 2, 281-296 (1978).

[4] Kaufmann, R., Hillenkamp, F., Wechsung, R., Heinen, H. J., and Schürmann, M., Laser Microprobe Mass Analysis, Achievements and Aspects, Proc. of Scanning Electron Microscopy, Washington, April 16-22 (1979).

[5] Burns-Bellhorn, M.S., Review of Secondary Ion Mass Spectrometry in Biological Research, Adv. Techn. in Biomed. Electron Microscopy, Gennaro, J. (ed.), Masson et Cie (1978).

[6] Kupka, K. D., Hillenkamp, F., Schiller, Ch., Aminosäure-Massenspektren, gewonnen mit einem Laser-Mikromassenanalysator, Proc. Deutsche Physik1. Ges. Annual Meeting, March 5-9 (1979).

[7] McCrone, W., and Delly, J. G., The Particle Atlas, Ann Arbor Science Publ. Ins. Mich. (1975).

[8] Morton, R. R. A., and McCarthey, C., The Omnicon Pattern Anaiysis System, The Microscope $23,239-260$ (1975).

[9] Cadle, R. D., The Measurement of Airborne Particles, John Wiley \& Sons, New York, NY (1975).

10] Mercer, T. T., Aerosol Technology in Hazard Evaluation, Academic Press, London, New York (1973).

11] Wieser, R., Wurster, R., and Seiler, H., About the Identification of Airborne Particles by Laser Induced Mass Spectroscopy, To be Published in Atmospheric Environment.

12] Zebel, G., and Hochrainer, R., Zur Messung der GröBenverteilung des Feinstaubes mit einem verbesserten Spektral impaktor, Staub, Reinhaltung, Luft 3, 91-95 (1972).

13] Abraham, J. L., Recent Advances in Pneumoconiosis - The Pathologist's Role in Etiologic Diagnosis, IAP Monograph No. 19, The Williams \& Wilkins Co., The International Academy of Pathology (1978).

14] DeNee, P. B., Abraham, J. L., and Gelderman, A. H., Scanning Electron Microscopy, Om Johari, and Corvin I (eds.), ITT Research Institute, Chicago, IL, 411-418 (1973).

15] Caplan, A., Thorax, 8 , 27-29 (1953).

16] Parkes, W. R., Occupational Lung Disorders, London, Butterworths (1974).

17] Berglund, R. N., and Liu, B. Y. A., Generation of Monodisperse Aerosol Standards, Environmental Science \& Technology $\underline{7}, 141-153$ (1973). 

ITLE AND SUBTITLE

Proceedings of the Particle Analysis Session of the

13th Annual Conference of the Microbeam Analysis

Society, held at Ann Arbor, Michigan, June 22, 1978 UTHOR(S)

Kurt F. J. Heinrich, Editor

5. Publication Date

Apri1 1980

6. Performing Organization Code

8. Performing Organ. Report No.

ERFORMING ORGANIZATION NAME AND ADDRESS

10. Project/Task/Work Únit No.

IATIONAL BUREAU OF STANDARDS

OEPARTMENT OF COMMERCE

11. Contract/Grant No.

VASHINGTON, DC 20234

SPONSORING ORGANIZATION NAME AND COMPLETE ADDRESS (Street, City, State, ZIP)

13. Type of Report \& Period Covered

Final

14. Sponsoring Agency Code

\section{SUPPLEMENTARY NOTES}

Library cf Congress Catalog Card Number: 80-600033

Document describes a computer program; SF-185, FIPS Software Summary, is attached.

ABSTRACT (A 200-word of less tactual summary of most signiticant information. If documeat includes a significant bibliography or iterature survey, mention it here.)

This document contains a series of invited papers on the subject of Particle Characterization. Part of the material presented here was presented orally at the Thirteenth Annual Conference of the Microbeam Analysis Society, at Ann Arbor, Michigan, on June 22, 1978. The publication describes microscopic and analytical techniques for the characterization of single microscopic particles. Applications of these techniques to problems of general interest are also included.

KEY WORDS (six to twelve entries; alphabetical order; capitalize only the first letter of the first key word unless a proper namo; separated by semicolons)

Biological fluids; electron microscopy; electron microprobe; ion probe; light microscopy; microanalysis; microdroplets; particles; particulate matter.

AVAILABILITY X. Unlimited

For Official Distribution. Do Hot Release to NTIS

X Order From Sup. of Doc., U.S. Government Printing Office, Wasinington, DC 20402 ,

Order From National Technical Information Service (NTIS), Springfield, VA. 22161

\begin{tabular}{|l|c|}
\hline $\begin{array}{l}\text { 19. SECURITY CLASS } \\
\text { (THIS REPORT) }\end{array}$ & $\begin{array}{c}\text { 21. NO. OF } \\
\text { PRINTED PAGES } \\
\text { UNCLASSIFIED }\end{array}$ \\
\hline $\begin{array}{l}\text { 20. SECURITY CLASS } \\
\text { (THIS PAGE) }\end{array}$ & 222 \\
UNCLASSIFIED & $\$ 6.50$ \\
\hline
\end{tabular}





\section{NBS TECHNICAL PUBLICATIONS}

\section{PERIODICALS}

URNAL OF RESEARCH-The Journal of Research of the ional Bureau of Standards reports NBS research and develop$\mathrm{t}$ in those disciplines of the physical and engineering sciences in ch the Bureau is active. These include physics, chemistry, ineering. mathematics, and computer sciences. Papers cover a ad range of subjects, with major emphasis on measurement hodology and the basic technology underlying standardization. $\supset$ included from time to time are survey articles on topics ely related to the Bureau's technical and scientific programs. a special service to subscribers each issue contains complete tions to all recent Bureau publications in both NBS and non5 media. Issued six times a year. Annual subscription: domestic foreign \$21.25. Single copy, \$3 domestic; \$3.75 foreign.

TE: The Journal was formerly published in two sections: SecA "Physics and Chemistry" and Section B "Mathematical nces."

MENSIONS/NBS-This monthly magazine is published to inin scientists, engineers, business and industry leaders, teachers, lents, and consumers of the latest advances in science and inology, with primary emphasis on work at NBS. The magazine hlights and reviews such issues as energy research, fire protec, building technology, metric conversion, pollution abatement, th and safety, and consumer product performance. In addiit reports the results of Bureau programs in measurement dards and techniques, properties of matter and materials, ineering standards and services, instrumentation, and matic data processing. Annual subscription: domestic \$11; ign $\$ 13.75$.

\section{NONPERIODICALS}

nographs-Major contributions to the technical literature on ous subjects related to the Bureau's scientific and technical acies.

adbooks-Recommended codes of engineering and industrial tice (including safety codes) developed in cooperation with insted industries, professional organizations, and regulatory ies.

cial Publications-Include proceedings of conferences spon$d$ by NBS, NBS annual reports, and other special publications ropriate to this grouping such as wall charts, pocket cards, and iographies.

lied Mathematics Series-Mathematical tables, manuals, and lies of special interest to physicists, engineers, chemists, ogists, mathematicians, computer programmers, and others tged in scientific and technical work

ional Standard Reference Data Series-Provides quantitative i on the physical and chemical properties of materials, comd from the world's literature and critically evaluated. eloped under a worldwide program coordinated by NBS under authority of the National Standard Data Act (Public Law 396).
NOTE: The principal publication outlet for the foregoing data is the Journal of Physical and Chemical Reference Data (JPCRD) published quarterly for NBS by the American Chemical Society (ACS) and the American Institute of Physics (AIP). Subscriptions, reprints, and supplements available from ACS, 1155 Sixteenth St. NW, Washington, DC 20056.

Building Science Series-Disseminates technical information developed at the Bureau on building materials, components, systems, and whole structures. The series presents research results, test methods, and performance criteria related to the structural and environmental functions and the durability and safety characteristics of building elements and systems.

Technical Notes_Studies or reports which are complete in themselves but restrictive in their treatment of a subject. Analogous to monographs but not so comprehensive in scope or definitive in treatment of the subject area. Often serve as a vehicle for final reports of work performed at NBS under the sponsorship of other government agencies.

Voluntary Product Standards-Developed under procedures published by the Department of Commerce in Part 10, Title 15, of the Code of Federal Regulations. The standards establish nationally recognized requirements for products, and provide all concerned interests with a basis for common understanding of the characteristics of the products. NBS administers this program as a supplement to the activities of the private sector standardizing organizations.

Consumer Information Series-Practical information, based on NBS research and experience, covering areas of interest to the consumer. Easily understandable language and illustrations provide useful background knowledge for shopping in today's technological marketplace.

Order the above NBS publications from: Superintendent of Documents, Government Printing Office, Washington, DC 20402.

Order the following NBS publications-FIPS and NBSIR's-from the National Technical Information Services, Springfield, VA 22161.

Federal Information Processing Standards Publications (FIPS PUB)-Publications in this series collectively constitute the Federal Information Processing Standards Register. The Register serves as the official source of information in the Federal Government regarding standards issued by NBS pursuant to the Federal Property and Administrative Services Act of 1949 as amended, Public Law 89-306 (79 Stat. 1127), and as implemented by Executive Order 11717 (38 FR 12315, dated May 11, 1973) and Part 6 of Title 15 CFR (Code of Federal Regulations).

NBS Interagency Reports (NBSIR)-A special series of interim or final reports on work performed by NBS for outside sponsors (both government and non-government). In general, initial distribution is handled by the sponsor; public distribution is by the National Technical Information Services, Springfield, VA 22161, in paper copy or microfiche form.

\section{BIBLIOGRAPHIC SUBSCRIPTION SERVICES}

following current-awareness and literature-survey bibliographies issued periodically by the Bureau:

ogenic Data Center Current Awareness Service. A literature surissued biweekly. Annual subscription: domestic $\$ 25$; foreign

sefied Natural Gas. A literature survey issued quarterly. Annual scription: \$20.
Superconducting Devices and Materials. A literature survey issued quarterly. Annual subscription: \$30. Please send subscription orders and remittances for the preceding bibliographic services to the National Bureau of Standards, Cryogenic Data Center (736) Boulder, CO 80303. 
U.S. DEPARTMENT OF COMMERCE

National Bureau of Standards

Washington, D.C. 20234

POSTAGE AND FEES PAID

OFFICIAL. BUSINESS

Penalty for Private Use, \$300
U.S. OEPARTMENT OF COMMERCE

COM-2 35

SPECIAL FOURTH-CLASS RATE

BOOK 